\title{
The role of Calcium Binding Protein 2 in synaptic sound encoding and hearing
}

\section{Dissertation}

\author{
for the award of the degree \\ "Doctor rerum naturalium" (Dr. rer. nat.) \\ of the Georg-August-Universität Göttingen
}

submitted by:

Maria Magdalena Picher

from:Vienna

Göttingen, 2015 
Members of the thesis committee:

Reviewer: Prof. Dr. Tobias Moser

Institute for Auditory Neuroscience, University Medical Center Göttingen

Reviewer: Prof. Dr. Fred Wolf

Theoretical Neurophysics, Max Planck Institute for Dynamics and Self-Organization

Prof. Dr. Thomas Dresbach

Department of Anatomy and Embryology, Center of Anatomy, University Medical Center Göttingen

Date of the oral examination: $2^{\text {nd }}$ of December, 2015 


\section{Declaration}

I hereby declare that this thesis has been written independently and with no other sources and aids than quoted.

Maria Magdalena Picher Göttingen, $2^{\text {nd }}$ of October, 2015 


\section{Contents}

1 Introduction 1

1.1 The auditory system . . . . . . . . . . . . . . . . . . 1

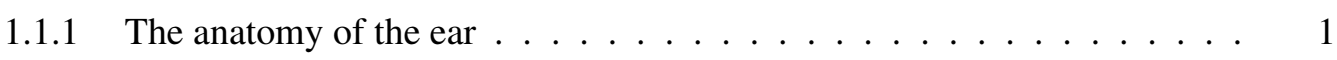

1.1.1.1 The outer ear . . . . . . . . . . . . . . . 1

1.1.1.2 The middle ear . . . . . . . . . . . . . . . 1

1.1.1.3 The inner ear . . . . . . . . . . . . . . . 2

1.1.2 Tonotopic organization and sound encoding in the organ of Corti . . . . . 4

1.1.2.1 Frequency decomposition in the cochlea . . . . . . . . . 4

1.1.2.2 Cochlear amplification . . . . . . . . . . . . 5

1.1.2.3 Sound encoding by inner hair cell synapses . . . . . . . . . 6

1.2 The inner hair cell ribbon synapse . . . . . . . . . . . . . . . . 7

1.3 Voltage-gated $\mathrm{Ca}^{2+}$ channels $\ldots \ldots \ldots \ldots \ldots$

1.3.1 The $\mathrm{Ca}_{V} \alpha 1$ subunit . . . . . . . . . . . . . . . 9

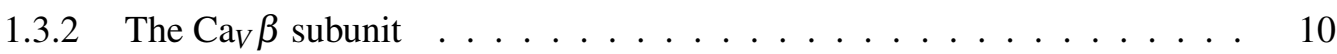

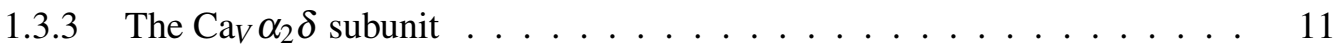

1.3.4 The $\mathrm{Ca}_{V} \gamma$ subunit . . . . . . . . . . . . . . . . . . . 11

1.3.5 Structure and function of $\mathrm{Ca}^{2+}$ binding proteins (CaBPs) . . . . . . 12

1.3.6 Negative feedback regulation of $\mathrm{Ca}^{2+}$ channels $\ldots \ldots \ldots 13$

1.3.6.1 Voltage-dependent inactivation (VDI) . . . . . . . . . 13

1.3.6.2 $\mathrm{Ca}^{2+}$-dependent inactivation (CDI) . . . . . . . . . 13

1.4 Genetic factors inducing sensorineural hearing impairment . . . . . . . . . . . 14

1.4.1 Impairment of the $\mathrm{K}^{+}$cycle . . . . . . . . . . . . . . . . . . . 14

1.4.2 Amplification deficits . . . . . . . . . . . . . . . . . . . 14

1.4 .3 Synaptopathies . . . . . . . . . . . . . . . . . . . . 15

1.5 Viral gene therapy as a tool to restore hearing . . . . . . . . . . . . . . 15

1.5.1 Hearing restoration in Vglut3 knock-out mice . . . . . . . . . . . . 15

1.5.2 Improved hearing in Tmc1 mutant mice . . . . . . . . . . . . . . . 16

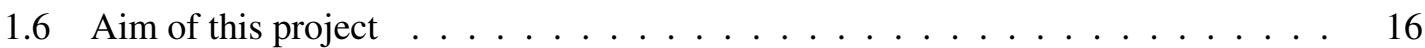

2 Materials and Methods 19

2.1 Cloning of pEGFP-N1-hCABP2 . . . . . . . . . . . . . . . . . . . . . 19

2.2 Generation of stable HEK293/hSK3-1 cells . . . . . . . . . . . . . . . . . 20

2.3 Electrophysiological recordings of transiently transfected HEK293 cells . . . . . 20

2.4 Generation of the Cabp2 $2^{\mathrm{LacZ} / \mathrm{LacZ}}$ mouse model $\ldots \ldots \ldots \ldots 21$

2.4.1 Long-Range PCR (LR-PCR) of ES cell (embryonic stem cell) clone genomic DNA . . . . . . . . . . . . . . . . . . . 21

2.4.2 Recombination strategy for Cabp2 $2^{\mathrm{LacZ} / \mathrm{LacZ}}$ mice $\ldots \ldots \ldots 22$ 
2.5 Isolation of the organ of Corti . . . . . . . . . . . . . . . . . . 22

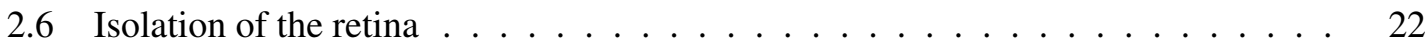

2.7 Protein isolation and Western Blotting . . . . . . . . . . . . . . . 22

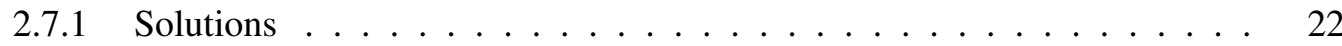

2.7.2 Western Blotting Procedure . . . . . . . . . . . . . . . 24

2.8 Reverse transcription (RT) of Cabp2 mRNA . . . . . . . . . . . . . . . . 25

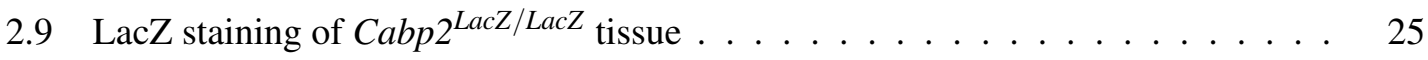

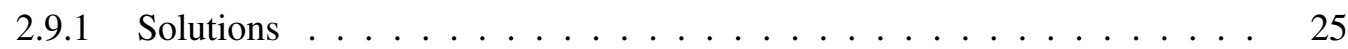

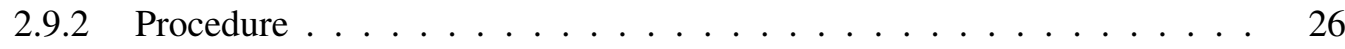

2.10 Immunohistochemistry . . . . . . . . . . . . . . . . . . . . 26

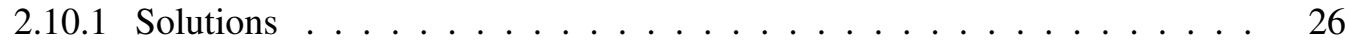

2.10 .2 Procedure for the organ of Corti . . . . . . . . . . . . . 26

2.10 .3 Procedure for retinal slices . . . . . . . . . . . . . . . . . . 27

2.11 Patch clamp recordings of inner hair cells . . . . . . . . . . . . . . 28

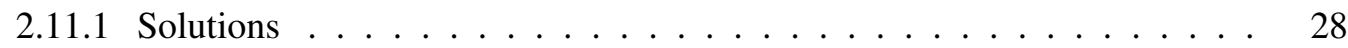

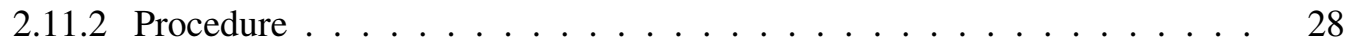

2.12 Extracellular recordings of spiral ganglion neurons _ . . . . . . . . . . . . . . 29

2.13 Systems physiology . . . . . . . . . . . . . . . . . . . . . . . . . . 29

2.13 .1 Electroretinography $($ ERGs) $\ldots \ldots \ldots$

2.13.2 Auditory brainstem responses (ABRs) . . . . . . . . . . . . . . . . . 29

2.13.3 Distortion product otoacoustic emissions (DPOAEs) $\ldots \ldots$. . . . . 30

2.14 Viral rescue of $C a b p 2^{L a c Z / L a c Z} \ldots \ldots \ldots \ldots \ldots$

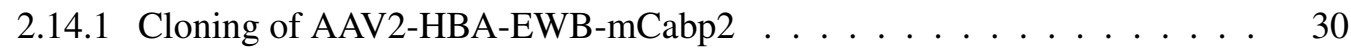

2.14.2 Embryonic transuterine otocyst injection of AAV2-HBA-EWB-mCabp2-

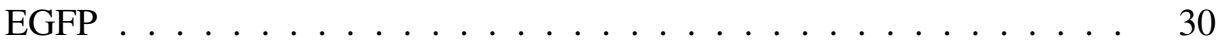

2.15 Data analysis . . . . . . . . . . . . . . . . . . 31

2.15.1 Current-Voltage curves (IV-curves) $\ldots \ldots \ldots 31$

2.15 .2 Inactivation . . . . . . . . . . . . . . . . . 32

2.15 .3 CDI recovery . . . . . . . . . . . . . . . . 32

3 Results $\quad 33$

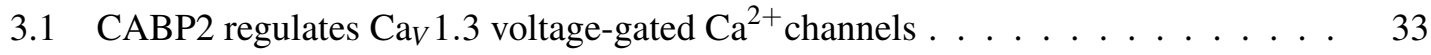

$3.2 C a b p 2^{\mathrm{LacZ} / \mathrm{LacZ}}$ as a model for DFNB93 $\ldots \ldots \ldots \ldots \ldots$

3.2.1 Indirect approach to detect Cabp2 expression in Cabp2 $2^{\mathrm{LacZ} / \mathrm{LacZ}}$ mice . . 39

3.2.2 Moderate hearing impairment in $\mathrm{Cabp} 2^{\mathrm{LacZ} / \mathrm{LacZ}}$ mice $\ldots . . . . . .41$

3.2.3 IHC development is not affected in the absence of functional Cabp2 . . . 41

3.2.4 Voltage-sensitivity of IHC Ca ${ }^{2+}$ influx is not altered in Cabp2 ${ }^{\mathrm{LacZ} / \mathrm{LacZ}}$ mice 43

3.2.5 $\mathrm{Ca}_{V} 1.3 \mathrm{Ca}^{2+}$ channel inactivation is increased in Cabp2 $2^{\mathrm{LacZ} / \mathrm{LacZ}}$ mice . . 46

II 
3.2.6 Cabp2-deficiency results in increased sustained exocytosis . . . . . . 5 50

3.2.7 Cabp2-deficiency impairs $\mathrm{Ca}^{2+}$ buffering in IHCs . . . . . . . . . . . . 52

3.2.8 Spiral ganglion neuron responses in $\mathrm{Cabp} 2^{\mathrm{LacZ} / \mathrm{LaCZ}}$ animals $\ldots . . .54$

3.2.9 Gene-therapeutic approach to rescue DFNB93 . . . . . . . . . . 57

4 Discussion $\quad 61$

$4.1 C a b p 2^{\mathrm{LacZ} / \mathrm{LacZ}}$ mice are a suitable animal model for DFNB93 $\ldots \ldots \ldots \ldots$. . . . 61

4.2 Cabp2 expression in $C a b p 2^{\text {LacZ } / L a c Z}$ animals . . . . . . . . . . . . . . . 62

4.3 Experiments in heterologous expression systems . . . . . . . . . . . . . 63

4.3.1 CABP2-wt increases the voltage-sensitivity and inhibits CDI of $\mathrm{Ca}_{V} 1.3$

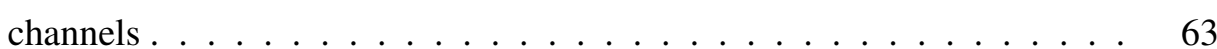

$4.4 \mathrm{IHC} \mathrm{Ca}{ }^{2+}$ channels display increased inactivation . . . . . . . . . . . . . 64

4.4.1 $\mathrm{Ca}^{2+}$-dependent inactivation is not affected in $\mathrm{Cabp} 2^{\mathrm{LacZ} / \mathrm{LacZ}} \ldots \ldots . .64$

4.4.2 Increased voltage-dependent inactivation in $\mathrm{Cabp}^{\mathrm{LacZ} / \mathrm{LacZ}}$ animals $\ldots .65$

4.4.3 Steady-state inactivation of $\mathrm{Ca}_{V} 1.3$ channels in Cabp2 $2^{\mathrm{LacZ} / \mathrm{LacZ}} \mathrm{IHCs}$. . 67

4.5 Cabp2 acts as a $\mathrm{Ca}^{2+}$ buffer in IHCs $\ldots \ldots \ldots$. . . . . . . . . . . 69

4.6 DFNB93 as another candidate of a channelopathy . . . . . . . . . . . . 72

4.7 Potential of virus mediated gene therapy to treat DFNB93 . . . . . . . . . . 72

5 Summary $\quad 74$

6 Bibliography $\quad 76$

7 Acknowledgements $\quad 94$

8 Appendix $\quad 96$

9 Abbreviations $\quad 98$

10 Publication List 100

11 Curriculum Vitae 102 


\section{List of Figures}

1.1 Gross anatomy of the outer, middle and inner ear $\ldots \ldots \ldots 2$

1.2 Anatomy of the inner ear . . . . . . . . . . . . . . . . 3

1.3 Frequency encoding at the basilar membrane . . . . . . . . . . . 5

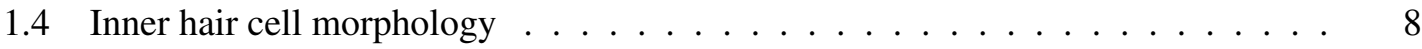

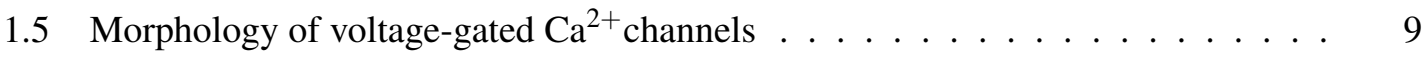

2.1 Knock-out strategy for the Cabp2 $2^{L a c Z / L a c Z}$ mouse line . . . . . . . . . . . . . 23

3.1 CABP2 modulates the biophysical properties of $\mathrm{Ca}_{V} 1.3_{42} \ldots \ldots \ldots \ldots$

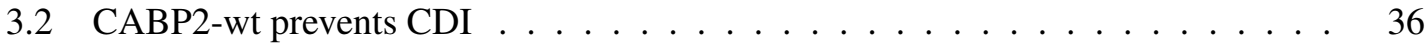

3.3 CABP2 modulates $\mathrm{Ca}_{V} 1.3_{42-A 2123 V}$ gating . . . . . . . . . . . . . 37

3.4 Cabp2-deficiency in $C a b p 2^{\text {LacZ/LacZ }}$ mice $\ldots \ldots \ldots \ldots \ldots$

3.5 Cabp2 is expressed in cochlear hair cells and in retinal cells . . . . . . . . . . . . . . 40

$3.6 C a b p 2^{\text {LacZ/LacZ }}$ mice exhibit progressive hearing loss $\ldots \ldots \ldots$. . . . . . . . 42

3.7 Scotopic ERGs in Cabp2 $2^{\mathrm{LacZ} / \mathrm{LacZ}}$ animals are normal . . . . . . . . . . . . . . 43

3.8 Cabp2-deficiency does not impair IHC integrity . . . . . . . . . . . . . . . 44

3.9 Normal current-voltage relationship in $\mathrm{Cabp}^{\mathrm{LacZ} / \mathrm{LacZ}}$ mice $\ldots \ldots \ldots . . \ldots .45$

$3.10 \mathrm{Cabp} 2^{\mathrm{LacZ} / \mathrm{LacZ}} \mathrm{IHC}$ show increased $\mathrm{Ca}_{V} 1.3$ inactivation $\ldots \ldots \ldots . \ldots . \ldots 47$

3.11 Recovery from inactivation is impaired in Cabp $2^{\mathrm{LacZ} / \mathrm{LacZ}} \mathrm{IHCs} \ldots \ldots . . . . . .49$

3.12 Cabp2-deficiency affects exocytosis . . . . . . . . . . . . . 51

$3.13 \mathrm{Cabp} 2$ supports $\mathrm{Ca}^{2+}$ buffering in IHCs . . . . . . . . . . . . . . . . . . . . . . . . . . . . . . . . . . 53

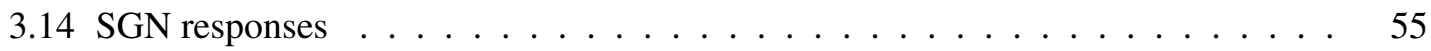

3.15 Rate-level functions are normal in $\mathrm{Cabp}^{\mathrm{LacZ} / \mathrm{LacZ}}$ animals $\ldots \ldots \ldots . \ldots 56$

3.16 AAV2/1-Cabp2-pEGFP transduces IHCs . . . . . . . . . . . . . . . 58

3.17 Viral transduction of AAV2/1-Cabp2-EGFP did not restore hearing . . . . . . . . 59 


\section{List of Tables}

2.1 LR-PCR thermo-cycle conditions. . . . . . . . . . . . . . . . . . . . . . 21

2.2 Implementation of immunostainings: Antibody host animals were chicken (ch), guinea pig $(\mathrm{gp})$, mouse $(\mathrm{m})$ and rabbit $(\mathrm{r}) . \ldots \ldots \ldots$

3.1 Biophysical properties of $\mathrm{Ca}_{V} 1.3$ in the presence or absence of CABP2-wt or CABP2-mutant expressed in HEK293/hSK3-1 cells . . . . . . . . . . . . 36

3.2 Inactivation of $\mathrm{Ca}_{V} 1.3$ in the presence or absence of CABP2-wt or CABP2-mutant expressed in HEK293/hSK3-1 cells . . . . . . . . . . . . . . . . . 37

3.3 Biophysical properties of $\mathrm{Ca}_{V} 1.3$ in IHCs . . . . . . . . . . . . . . . 46

3.4 Inactivation in $\mathrm{Cabp}^{+/+}$and Cabp2 ${ }^{\mathrm{LacZ} / \mathrm{LacZ}} \mathrm{IHCs}$ of young p15-20 animals . . 47

8.1 Cloning/RT-PCR primer sequences used throughout this study . . . . . . . . . 96

8.2 Sequencing primers used throughout this study $\ldots \ldots \ldots$ 



\section{Introduction}

\subsection{The auditory system}

Hearing is a unique sensory feature in the animal kingdom providing the individual with acoustic information about the surrounding environment. Animals are dependent on sound to communicate and localize prey or predators. For humans, hearing facilitates communication and social interaction, the identification of threats and enjoyment by listening to music, movies or plays. The perceptible auditory spectrum of mammals includes frequencies ranging from $20 \mathrm{~Hz}$ to more than $100 \mathrm{kHz}$ with humans being susceptible for $20 \mathrm{~Hz}$ to $20 \mathrm{kHz}$. Another remarkable feature of hearing is the ability of perceiving sound intensities over a wide dynamic range from 0 to 120 $\mathrm{dB}$, thus six orders of magnitude. In land-born vertebrates, the hearing process is initiated by the ear, the auditory end organ that converts air-born sound vibrations into fluid-transmitted sound pressure and finally into a neural code. To convey information about pitch, intensity and location, the auditory system takes advantage of place, rate, and temporal codes.

\subsubsection{The anatomy of the ear}

The mammalian ear consists of three compartments: i) the outer ear, ii) the middle ear and iii) the inner ear.

\subsubsection{The outer ear}

The outer ear is comprised of the pinna (auricula), an extending cup-like structure that first receives air-borne sound waves and funnels them into the ear canal (acoustic meatus). Dependent on the species the pinna can either be directly or indirectly oriented (i.e. voluntary vs. involuntary head movement) and supports sound localization in both, the horizontal and the vertical plane of a hemifield. The directionality of the pinna furthermore prevents front-back confusion and by its mere anatomy acts as a primary filter ((Heffner and Heffner, 2008); Figure 1.1A).

\subsubsection{The middle ear}

The middle ear forms a partition between the air-filled outer ear and the fluid-filled cochlea. It consists of the outermost tympanic membrane followed by the three ossicles, the malleus (hammer), incus (anvil) and stapes (stirrup), that transmit sound-induced vibration of the tympanic membrane onto the membrane of the oval window of the cochlea. Its main function is to convert airborne sound waves received by vibrations of the tympanic membrane into sound pressure waves in the fluid-filled space of the cochlea ultimately delivered by the stapes, the innermost of the three ossicles. This anatomical framework is necessary for transmitting sound across the airfluid boundary, since otherwise the high impedance medium (fluid) would reflect the sound from 
a low impedance medium (air). The middle ear not only preserves the received sound energy, but also increases the sound pressure 200 fold by using the lever action of the three ossicles to focus the energy from the relatively large tympanic membrane onto the small-diameter oval window of the cochlea ((Purves et al., 2001); Figure 1.1A).

A

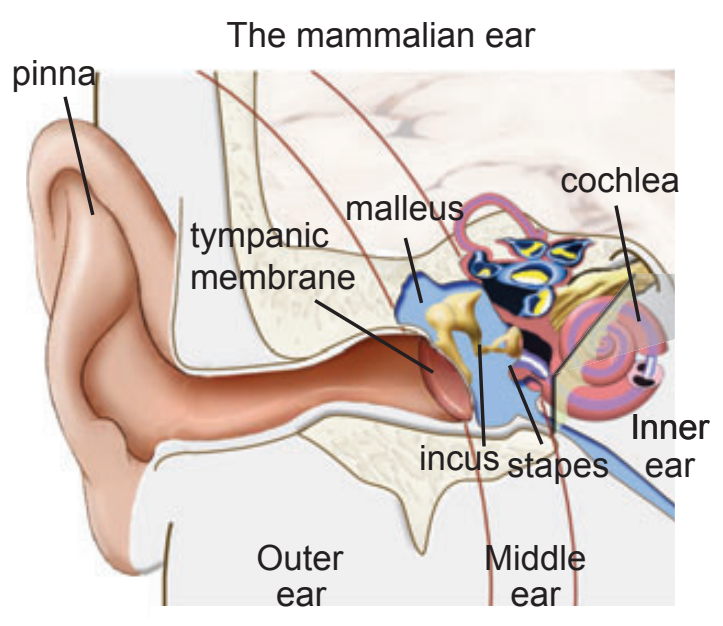

B

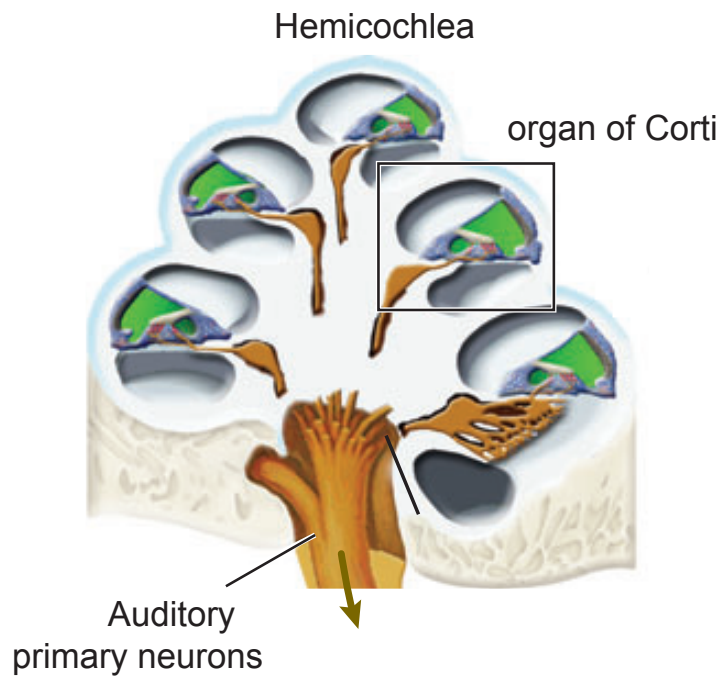

Figure 1.1: (A) Gross anatomy of the outer, middle and inner ear . (B) Cross-section through the cochlea along the modiolus. The organ of Corti is highlighted by the gray rectangle (modified from (Safieddine et al., 2012)).

\subsubsection{The inner ear}

After sound waves have passed the outer and middle ear, they arrive at the inner ear. The inner ear is comprised of the cochlea, a snail-like structure harboring the organ of Corti, i.e. the auditory sensory epithelium (Figure 1.2A). The cochlea has three compartments: (i) scala vestibuli, (ii) scala media and (iii) scala tympani. At the apex of the cochlea, scala tympani and scala vestibuli are connected through the so-called helicotrema (Figure 1.2B). Due to the helicotrema, sound pressure waves are propagating through the scala tympani along the cochlea to the scala vestibuli terminated at the round window. Scala tympani and scala vestibuli are both filled with perilymph, a fluid with similar ionic composition as cerebrospinal fluid with a $\mathrm{K}^{+}$concentration of approximately $5 \mathrm{mM}$. However, the scala media is filled with endolymph, which contains similar to intracellular fluid: a high concentration of $\mathrm{K}^{+}$(approximately $160 \mathrm{mM}$ ) and low concentrations of $\mathrm{Na}^{+}$and $\mathrm{Ca}^{2+}$ ions (Dallos, 1996; Purves et al., 2001). The partition between scala tympani and media is formed by the basilar membrane, a specialized basement membrane comprised of type II (Cosgrove et al., 1996) and type IV collagen and the reticular lamina (Dreiling et al., 2002; Tsuprun and Santi, 1999). Apically, the organ of Corti is flanked by the tectorial membrane, another extracellular matrix component consisting of an ear specific glycoprotein matrix, $\alpha$ and 
$\beta$-tectorin, otogelin and mostly type II collagen ((Goodyear and Richardson, 2002; Gueta et al., 2007; Richardson et al., 2008), reviewed in (Mann and Kelley, 2011)).

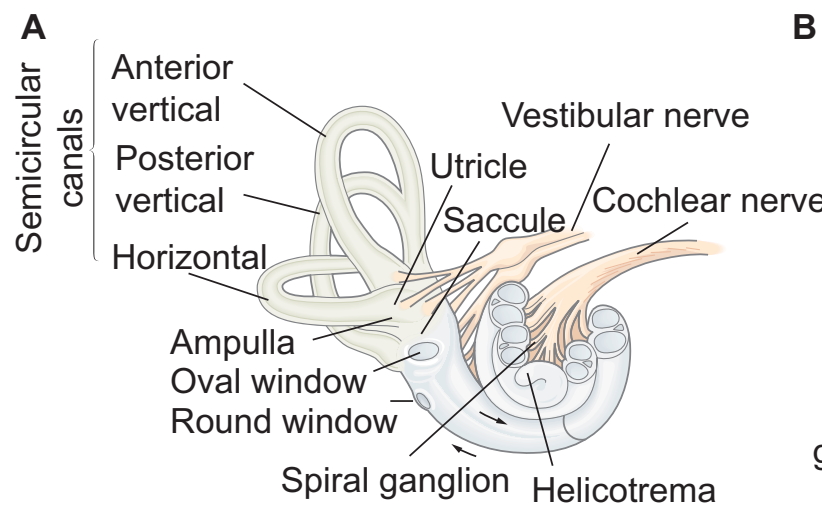

B

C
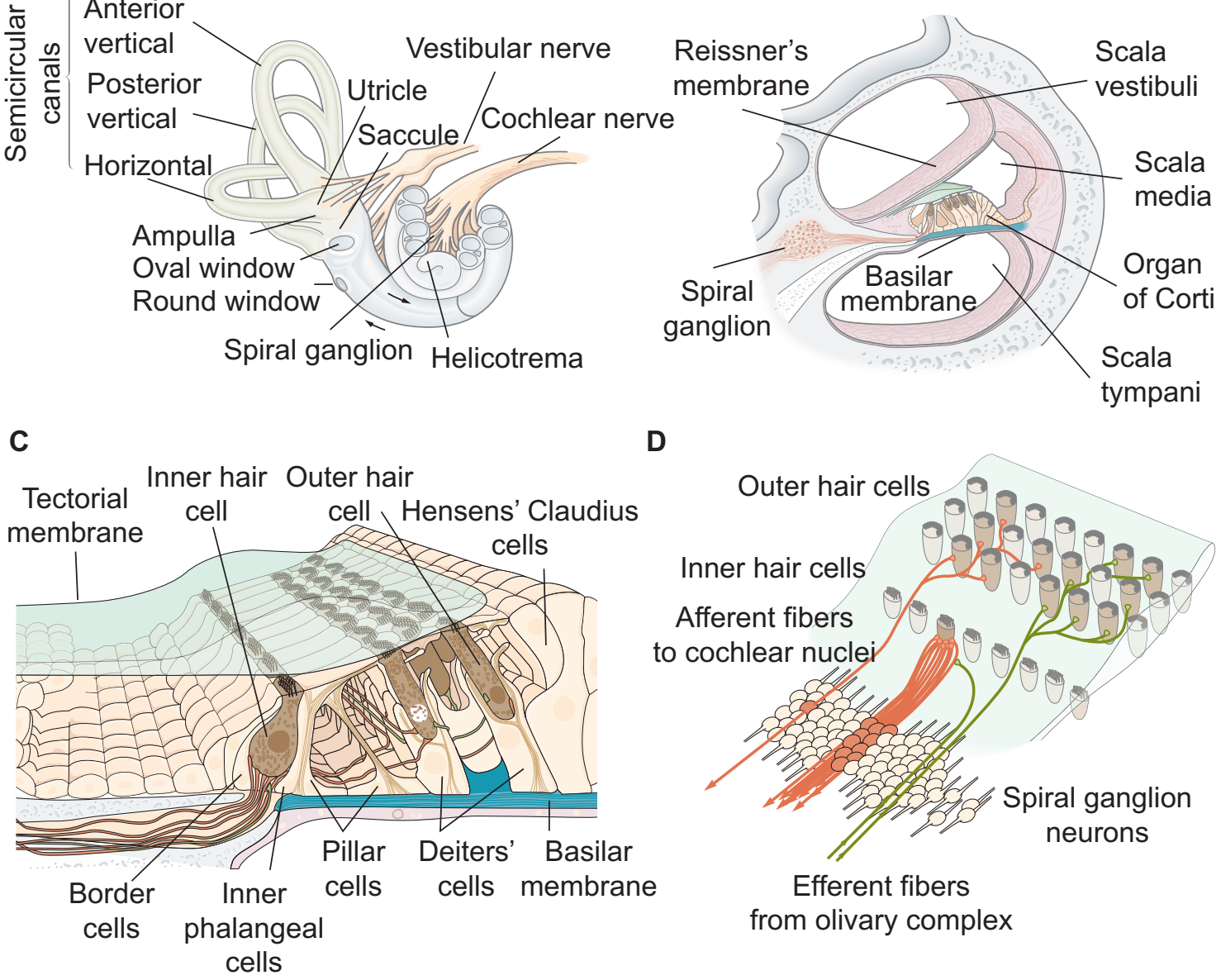

D

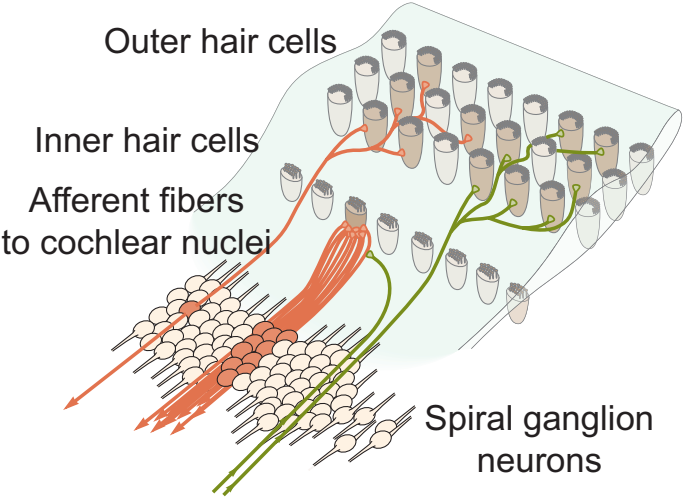

Efferent fibers from olivary complex

Figure 1.2: Anatomy of the inner ear: (A) Schematic drawing of the cochlea containing the semicircular canals, utricle, saccule and ampulla of the vestibular organ and the auditory organ. (B) Cross-section through the cochlear snail highlighting the three partitions, with the organ of Corti housing in the scala media. (C) Cellular mosaic-like structure of the organ of Corti outlining the characteristic sensory and supporting cells. Tectorial and basilar membrane are shown in blue. (D) Afferent (orange) and efferent (green) innervation of inner and outer hair cells. While multiple SGNs innervate IHC, multiple OHC are innervated by one fiber. Modified from (Kandel et al., 2000)

The organ of Corti exhibits two distinct populations of sensory hair cells, three-four rows of outer hair cells (OHC) and a single row of inner hair cells (IHC), that are innervated by type I and type II spiral ganglion neurons. The sensory hair cells are crowned with 50-100 stereocilia at their apex, which in fact are microvilli forming a well defined bundle. OHC are separated from one another by Deiters' cells and are characterized by electromotile responses upon depolarization (Figure 1.2C), where they translate depolarization of sound-induced $\mathrm{K}^{+}$ion influx into a reduction 
in cell length ((Frolenkov et al., 1998); for more details see Section 1.1.2.2 below). The longest outermost row of stereocilia of every single $\mathrm{OHC}$ reaches through the lumen and is anchored in the tectorial membrane (Kamiya et al., 2014). While only $5 \%$ of afferent fibers innervate OHC, more than $90 \%$ contact IHC, the genuine sensory receptors of the organ of Corti that convey all auditory information to the brain. Anatomically they are separated from the $\mathrm{OHC}$ by the tunnel of Corti, which is formed by outer and inner pillar cells and are isolated from neighboring IHC by surrounding inner phalangeal and border cells (Kandel et al., 2000).

The hair bundles of IHCs and OHCs are mechanosensory organelles assembled in a staircase array. Stereocilia are connected via different kinds of molecular linkers to allow coherent motionbundle defection upon sound stimulation ((Verpy et al., 2011); Figure 1.4B). For example, two adjacent first and second row stereocilia are connected by tip-link complexes. These tip-links are comprised of two different cadherin molecules, cadherin 23 (CDH23; (Siemens et al., 2004)) and protocadherin 15 (PCH15; (Ahmed et al., 2006)) that interact via their N-termini to establish a molecular connection. Here, while $\mathrm{CDH} 23$ was suggested to extend from the side of the taller stereocilium, PCH15 was located at the tip of the shorter stereocilium (Figure 1.4B $\mathrm{B}_{i}$ ). Upon stereociliar deflection, tension of the tip-link increases and is thought exert force on the mechanoelectrical transduction (MET) channels (Kazmierczak et al., 2007). This leads to increased MET channel opening probability that depolarizes the cells and ultimately elicits two distinct responses in IHCs and OHCs (i.e. synaptic release in IHCs vs. electromotility in OHCs; (Garcia et al., 1998; Holt et al., 2002; Sakaguchi et al., 2009)).

\subsubsection{Tonotopic organization and sound encoding in the organ of Corti}

\subsubsection{Frequency decomposition in the cochlea}

Starting in the sensory organ, frequency or tonotopic maps are established throughout the auditory pathway. In the coiled mammalian cochlea, high frequency sounds are detected at the base of the organ of Corti and low frequency sounds at the apex. This results from the micromechanical properties of the organ of Corti and basilar membrane that varies along the cochlear duct. At the base, the basilar membrane is thinner and narrower and gradually becomes wider and thicker with decreasing frequency specificity (Dallos, 1996). The amplitude of this traveling wave reaches a maximum at a certain position of the basilar membrane (Figure 1.3A) where a given frequency best vibrates the cochlear partition. Stereociliar deflection is maximal at this particular position and elicits synaptic release from adjacent IHCs and cochlear amplification through OHC electromotility. Therefore, the cochlea acts as a frequency analyzer and is able to decompose complex sounds ((Russell and Sellick, 1977); reviewed in (Mann and Kelley, 2011)). Although essential, the basilar membrane is not accounting for tonotopy all by itself. To achieve this remarkable frequency resolving power of the cochlea, the interplay of multiple factors is crucial to sharpen the 
traveling wave. For example, (i) graded changes of tectorial membrane organization and stiffness along the cochlea duct (Gummer et al., 1996; Richardson et al., 2008), (ii) sound encoding of IHCs by a parabolically distributed numbers of ribbon synapses (Meyer et al., 2009), as well as (iii) changing expression patterns of $\mathrm{Ca}^{2+}$-dependent large $\mathrm{K}^{+}$conductance (BK) channels and $\mathrm{Ca}^{2+}$ buffers in IHCs along the tonotopic axis (Imamura and Adams, 1996), (iv) an amplification gradient due to increasing OHC length from base to apex (Engel et al., 2006) and (v) alteration in SGN morphology and frequency encoding (Liberman and Oliver, 1984).

A

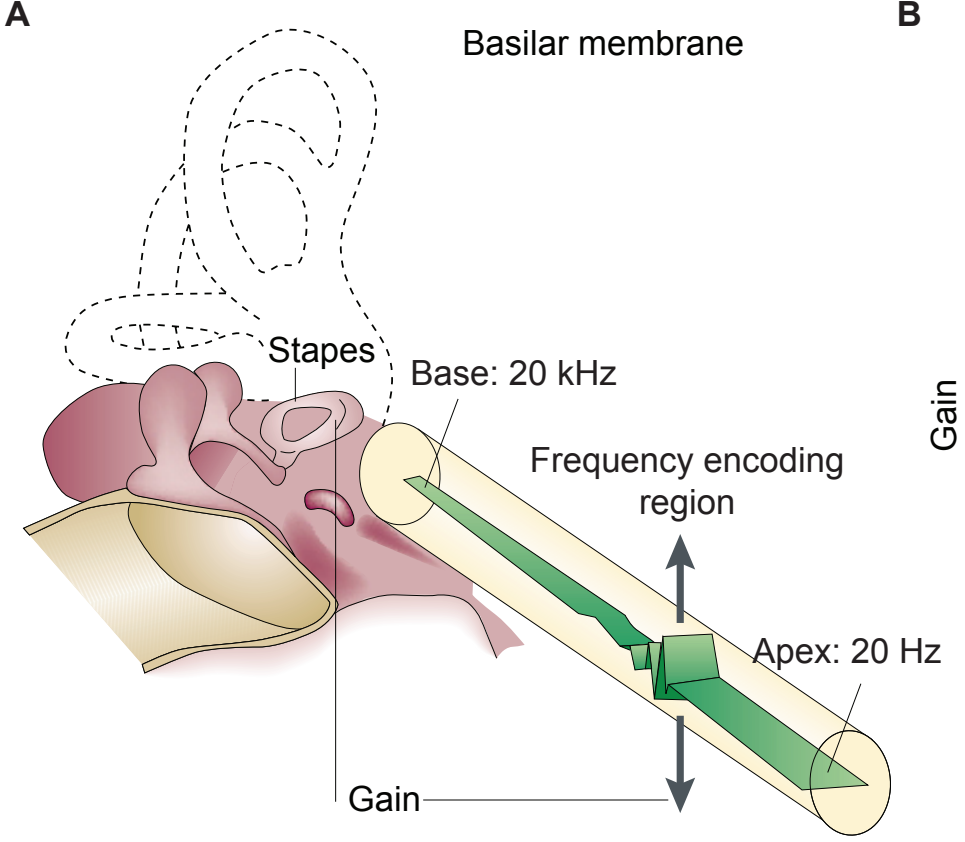

B

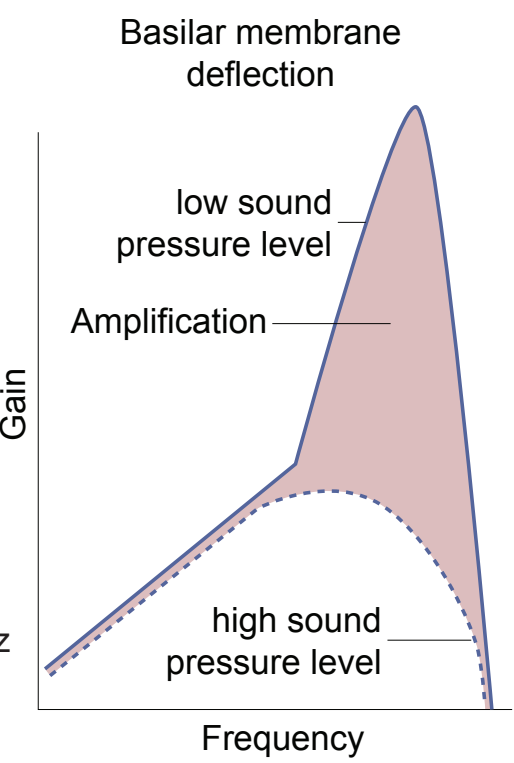

Figure 1.3: Frequency encoding at the basilar membrane: (A) Illustration of the uncoiled basilar membrane, that is narrower and thinner at the base of the cochlea where high frequency sounds are encoded. Toward the apex of the cochlea decreasing frequencies are perceived, basilar membrane increases in width and thickness. The peak of a traveling wave along the basilar membrane is highlighted by perpendicular arrows. In the organ of Corti, the region of maximal stereocilia deflection triggers a response in the neighboring hair cells (adapted from (Dallos and Fakler, 2002)). (B) Exemplary sound gain at a given frequency comparing the gain in presence (filled line) or absence (dashed line) of OHC induced sound amplification (adapted from (Dallos and Fakler, 2002)).

\subsubsection{Cochlear amplification}

Cochlear amplification is a process most pronounced in the mid-frequency region of the organ of Corti and is mainly governed by $\mathrm{OHC}$ function but also tectorial membrane flexibility (Legan et al., 2000).

As mentioned above, $\mathrm{OHC}$ translate depolarization due to cation influx (primarily $\mathrm{K}^{+}$) into 
changes in $\mathrm{OHC}$ length in a precisely timed microscopically visible contraction-expansion manner (Ashmore, 1987). Provided with this unique trait, OHCs act as sound wave amplifiers with a gain reaching as much as 1000 fold signal increase ((Purves et al., 2001); Figure 1.3B). The molecular motor that drives OHC motility was suggested to be prestin (Oliver et al., 2001; Zheng et al., 2000), a protein closely related to anion-transporters. The exact mechanism of electromotility is under current investigation, one concept is that prestin uses monovalent anions as extrinsic voltage sensor and reacts with elongation upon anion translocation ((Oliver et al., 2001), reviewed in (Dallos and Fakler, 2002)). This precisely timed contraction-elongation cycle of OHCs translates into mechanical amplification and refinement of the traveling wave along the basilar membrane resulting in increased acoustic sensitivity and frequency selectivity (Dallos and Fakler, 2002).

\subsubsection{Sound encoding by inner hair cell synapses}

IHCs are the primary sensory cells in the cochlea that convert sound vibration into electrical signal, resulting in presynaptic glutamate release. These cells depolarize upon cation influx (primarily $\mathrm{K}^{+}$) through MET channels, which activates voltage-gated $\mathrm{Ca}^{2+}$ channels (VGCC) at the IHC active zone (AZ). The resulting $\mathrm{Ca}^{2+}$ influx is detected by the vesicular $\mathrm{Ca}^{2+}$ sensors and triggers exocytotic release of glutamate onto postsynaptic bouton-like terminals of SGNs (Figure 1.4A). Here, via activation of $\alpha$-amino-3-hydroxy-5-methyl-4-isoxazole propionic acid (AMPA) receptors (Glowatzki and Fuchs, 2002; Matsubara et al., 1996), SGNs depolarize, ultimately eliciting an action potential (AP) that propagates along the eighth vestibulocochlear nerve and is transmitted via the auditory brainstem to the brain.

Pre- and postsynaptic factors shape the sound intensity encoding properties of SGNs. Based on the findings from extracellular SGN recordings in the cochlear nucleus $(\mathrm{CN})$ of cats, three populations of auditory nerve fibers were previously identified. These fibers were classified by (i) their spiking activity without sound stimulation (i.e. spontaneous rate), (ii) sound intensity thresholds in response to pure tones presented at the characteristic frequency of a fiber and (iii) the dynamic range of spiking activity in response to increasing sound intensity (rate-level function) (Liberman, 1978). One population of fibers was characterized by low spontaneous spiking rate with high sound intensity thresholds and a broad dynamic range. The second population of SGNs exhibited high spontaneous rate firing with low thresholds and a narrower dynamic range. Finally, medium spontaneous rate fibers had intermediate properties of high and low spontaneous fibers (Taberner and Liberman, 2005). Interestingly, these SGN populations innervate IHC synapses in organized polarity, with high spontaneous rate fibers primarily contacting IHCs at the abneural side (pillar side) and low spontaneous rate fibers preferentially located at the neural side (facing the modiolus) (Liberman, 1982). To date, pre- and postsynaptic mechanisms were suggested to contribute to the heterogeneous firing behavior of SGNs. Here, larger presynaptic ribbons and $\mathrm{Ca}^{2+}$ channel clusters can be observed at the neural side of low spontaneous rate fibers (Frank 
et al., 2009), while larger AMPA receptor clusters with increased bouton size preferentially occur at the abneural side of high spontaneous rate fibers (Liberman et al., 2011).

\subsection{The inner hair cell ribbon synapse}

IHC synapses are required for sound encoding and this involves translating stereociliar deflection into presynaptic glutamate release in IHCs. At the basolateral region, IHCs express 12 - 15 AZ, each of them provided with a ellipsoid presynaptic ribbon complex projecting onto one single SGN afferent fiber (Meyer et al., 2009). These specialized ribbon synapses are predominantly found in sensory cells, apart from cochlear hair cells also in photoreceptors and retinal bipolar cells, where signaling requires high rates of sustained release. Each ribbon tethers approximately $70-200$ synaptic vesicles, is primarily comprised of RIBEYE and piccolino (Regus-Leidig et al., 2013; Schmitz et al., 2000) and is anchored to the AZ by the scaffold protein bassoon (Dick et al., 2003; Khimich et al., 2005). RIBEYE is a self-assembling protein consisting of an A- and a B-domain, the latter has the same structure as the transcription factor Ctbp2 (Magupalli et al., 2008; Schmitz et al., 2000). The exact function of the ribbon complex is under current debate, but has been suggested to serve as a conveyor belt that enables rapid delivery of vesicle to the presynaptic release sites (Bunt, 1971; Graydon et al., 2014) and also regulates synchronous vesicle fusion either by acceleration or inhibition of vesicle transportation (Fuchs, 2005; Graydon et al., 2011; Jackman et al., 2009; Parsons and Sterling, 2003). Moreover, it promotes presynaptic $\mathrm{Ca}^{2+}$ channel and vesicular release sites and organizes the clustering of $\mathrm{Ca}^{2+}$ channels at the AZ (Frank et al., 2010; Khimich et al., 2005). Ribbon synapses use vesicles from two pools: (i) the fast responding readily releasable vesicle pool (RRP), likely composed by vesicles that are directly beneath the ribbon as well as membrane proximal vesicles and (ii) the sustained releasable pool (Figure 1.4C). The molecular composition of IHC presynaptic AZs show significant differences from that of conventional synapses (Pangršič et al., 2012; Wichmann and Moser, 2015). For example, the common vesicular glutamate transporters 1 and 2 (Vglut1, Vglut2) are lacking, but instead the unconventional Vglut3 mediates glutamate loading at IHC synapses (Seal et al., 2008).

Moreover, synaptic transmission apparently operates in the absence of SNARE proteins, such as SNAP-25, syntaxin-1, synaptobrevin-1 or synaptobrevin-2 (Nouvian et al., 2011). Furthermore, the conventional $\mathrm{Ca}^{2+}$ sensors of vesicle fusion, the $\mathrm{C}$-domain proteins synaptotagmin I and II are not expressed (Safieddine and Wenthold, 1999). Instead, another C2-domain protein, namely otoferlin plays an essential role in IHC presynaptic release and also hearing (Yasunaga et al., 1999). Otoferlin is a member of the ferlin protein family and like other ferlins, is characterized by six C2-domains, most of which are able to bind $\mathrm{Ca}^{2+}$ (except $\mathrm{C} 2 \mathrm{~A}$ domain; (Helfmann et al., 2011; Johnson and Chapman, 2010; Ramakrishnan et al., 2009)). Therefore, otoferlin is suggested to act as the $\mathrm{Ca}^{2+}$ sensor, required for exocytosis at IHC ribbon synapses (Roux et al., 2006). In addition, otoferlin is required for vesicle replenishment possibly through promoting 
A

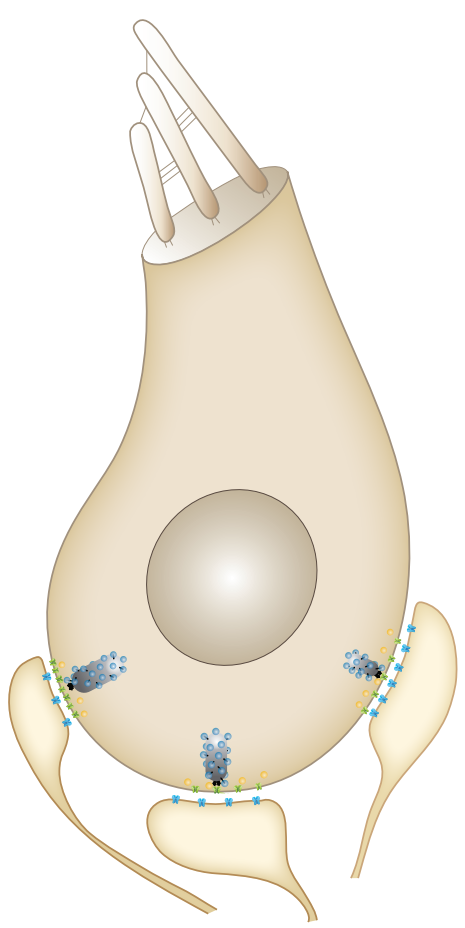

B

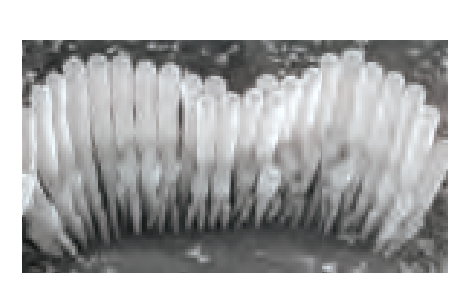

C

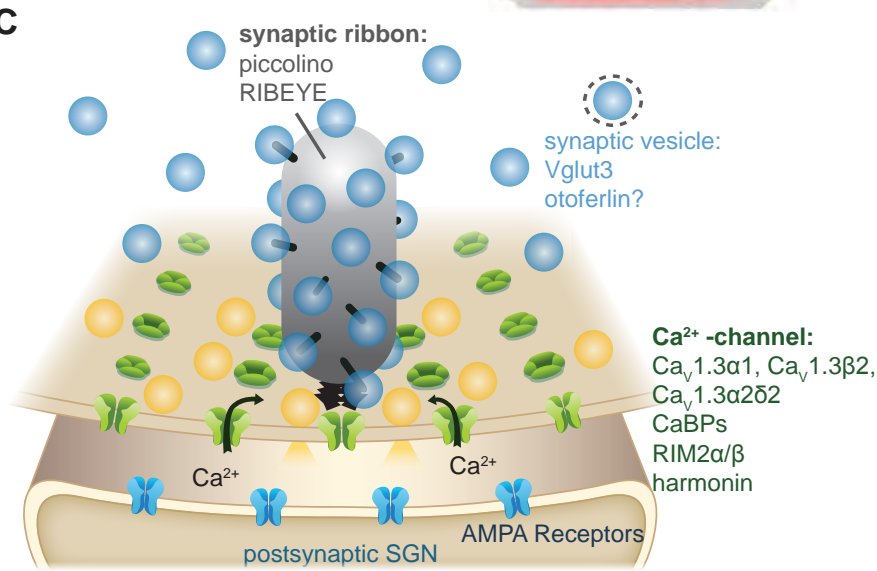

$B_{i}$

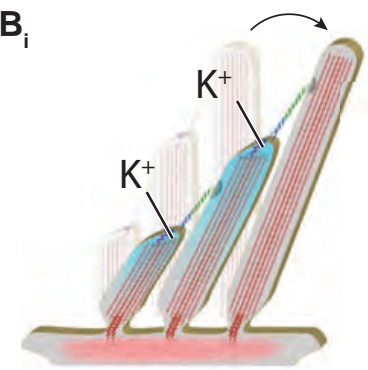

synaptic

Vglut3

toferlin?

Ca $1.3 \alpha 2 \delta 2$

CaBPs

RIM2 $\alpha / \beta$

postsynaptic SGN

Figure 1.4: Inner hair cell morphology: (A) Multiple ribbon synapses are expressed at the base of IHC, each of them individually projecting onto one SGN. (B) In the electron micrograph two major rows of stereocilia bundles at the apex of IHC are shown $\left(\mathrm{B}_{i}\right)$ with a schematic drawing of the actin meshwork in every stereocilium and respective $\mathrm{K}^{+}$ion influx upon deflection. Tip-link complexes between adjacent stereocilia are shown in color: $\mathrm{CDH} 23$ in green and PCH15 in blue. (C) IHC ribbon synapse with the ribbon in grey and the $\mathrm{Ca}_{V} 1.3$ channel stripes in green.

priming, while conventional priming factors of the mammalian uncoordinated 13 (Munc13) and $\mathrm{Ca}^{2+}$-dependent activator proteins for secretion (CAPS) families are absent (Pangršič et al., 2010; Vogl et al., 2015). The predominant $\mathrm{Ca}^{2+}$ channels at IHC ribbon synapses are $\mathrm{Ca}_{V} 1.3 \mathrm{VGCC}$, that account for $90 \%$ of the total $\mathrm{Ca}^{2+}$ current and are organized in stripe-like clusters at the $\mathrm{AZ}$ beneath the ribbon (Brandt et al., 2003; Frank et al., 2010; Platzer et al., 2000; Wong et al., 2014). $\mathrm{Ca}_{V} 1.3$ channels in IHC characteristically open at rather negative potentials and show only mild inactivation (Koschak et al., 2001). These channels are stabilized at the AZ by interaction with scaffolding proteins, such as Rab-3 interacting molecules $2 \alpha$ and $2 \beta$ (RIM2 $\alpha$ and RIM2 $\beta$ (Jung et al., 2015b)); and bassoon and /or the ribbon (Frank et al., 2010). Due to the indefatigable $\mathrm{Ca}^{2+}$ influx mediated by $\mathrm{Ca}_{V} 1.3$ channels, IHC are packed with $\mathrm{Ca}^{2+}$ buffers such as calretinin, calbindin and parvalbumin that prevent excessive $\mathrm{Ca}^{2+}$ spread, to constrain $\mathrm{Ca}^{2+}$ induced exocytosis at the AZ (Pangršič et al., 2015) (ribbon synapses reviewed in (Nouvian et al., 2006; Sterling and Matthews, 2005; Wichmann and Moser, 2015)). 
A

$\mathrm{Ca}_{\vee} \mathrm{a} 1$

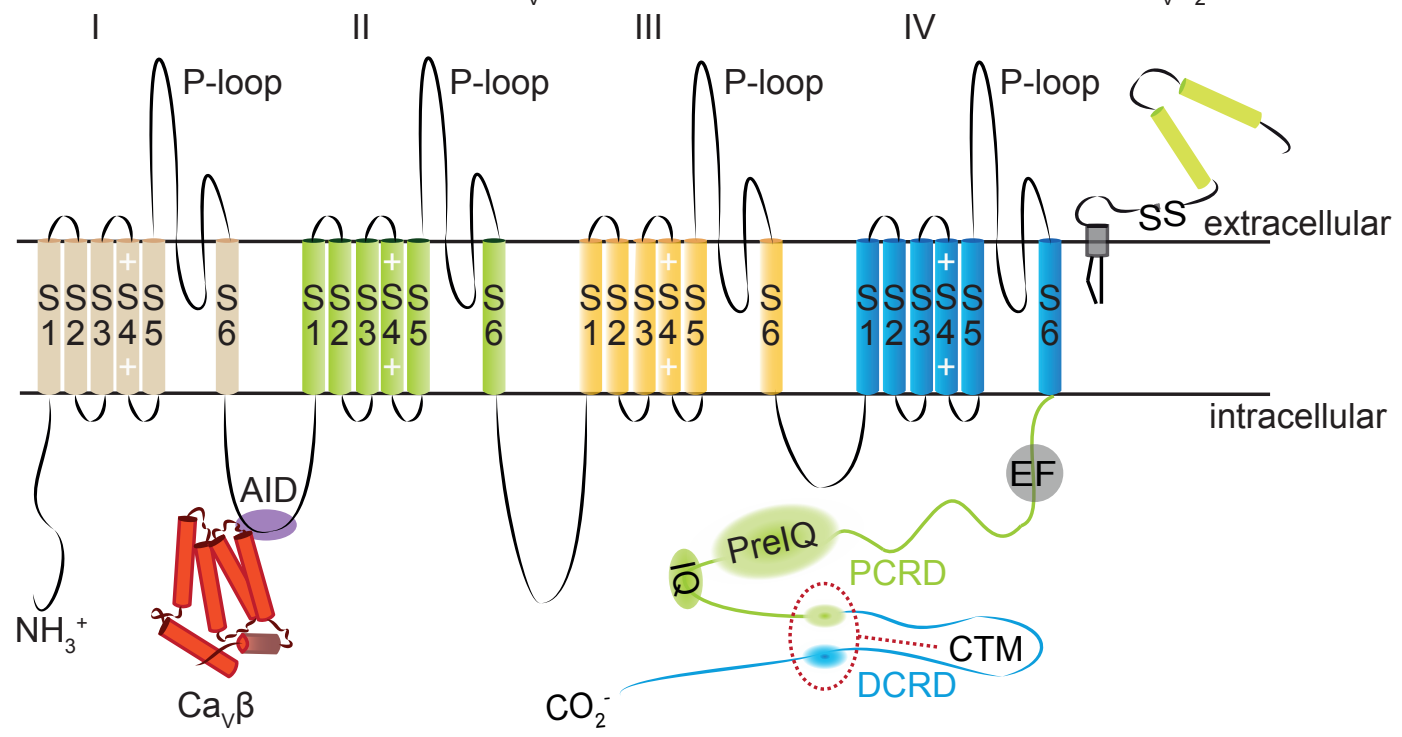

Figure 1.5: (A) Schematic drawing of VGCC morphology. Pore forming $\mathrm{Ca}_{V} \alpha 1$ subunit is presented in its elongated form with four homologous domains (I-IV) and the six transmembrane segments per domain (S1-S6). $\mathrm{Ca}_{V} \alpha_{2} \delta$ is linked to the membrane by a GPI-anchor and the cytosolic $\mathrm{Ca}_{V} \beta$ is in proximity of the alpha interaction domain (AID) within the I-II linker region.

\subsection{Voltage-gated $\mathrm{Ca}^{2+}$ channels}

VGCC possess the ability to convey changes in membrane potential into $\mathrm{Ca}^{2+}$ influx in various cell types, i.e. nerve, muscle, some endocrine and sensory cells. Elevations in intracellular $\mathrm{Ca}^{2+}$ levels trigger $\mathrm{Ca}^{2+}$-dependent signaling cascades and are crucial for excitation-secretion coupling , muscle excitation-contraction coupling, cell division, migration and differentiation. Moreover, loose regulation of VGCC gating can induce apoptosis due to $\mathrm{Ca}^{2+}$ toxicity. Therefore, VGCC are regulated by sophisticated feedback mechanisms to control intracellular $\mathrm{Ca}^{2+}$ levels. VGCC are classified into L-, N-, P/Q-, R- and T-types dependent on their subunit composition, electrophysiological and pharmacological properties and are comprised of a principal $\mathrm{Ca}_{V} \alpha 1$ subunit and 2-3 auxiliary subunits $\mathrm{Ca}_{V} \beta, \mathrm{Ca}_{V} \alpha_{2} \delta$ and $\mathrm{Ca}_{V} \gamma$ (Figure 1.5A; reviewed in (Catterall, 2011; Minor and Findeisen, 2010; Neely and Hidalgo, 2014)).

\subsubsection{The $\mathrm{Ca}_{V} \alpha 1$ subunit}

The $\mathrm{Ca}_{V} \alpha 1$ subunit is the pore forming subunit of VGCC is a polypeptide chain and comprised of four homologous repeats (I-IV) bridged by cytoplasmic loops. Every repeat has six transmembrane segments (S1-S6) of separate functions. To be able to respond to changes in membrane potential, VGCC are provided with a voltage sensor located in segment S4, which is comprised of four positively charged amino acids. $\mathrm{Ca}^{2+}$ ions are able to enter the cell through the channel 
pore, formed by segment $\mathrm{S} 6$ of every homologous repeat. Ion selectively and $\mathrm{Ca}^{2+}$ conductance is regulated by the signature locus, which is comprised of the $\mathrm{P}$ - or reentrant loop, each of them contributing with one negatively charged amino-acid. The I-II linker region is crucial for $\mathrm{Ca}_{V} \alpha 1$ subunit trafficking to the membrane (Gebhart et al., 2010) and houses the alpha interaction domain (AID), the high affinity binding region for the $\mathrm{Ca}_{V} \beta$ subunit. The pronounced $\mathrm{N}$-terminal tail of the $\mathrm{Ca}_{V} \alpha 1$ subunit bares multiple protein binding regions, that fine-tune channel gating and support channel interaction with synaptic scaffolding proteins (Figure 1.5A; (Jenkins et al., 2010; Zhang et al., 2005); reviewed in (Catterall, 2011; Zuccotti et al., 2011)).

As mentioned above, synaptic transmission at IHC ribbon synapses is predominantly regulated by $\mathrm{Ca}_{V} 1.3 \mathrm{Ca}^{2+}$ channels of the L-type $\mathrm{Ca}^{2+}$ channel (LTCC) family (Brandt et al., 2003). In recent years, multiple $\mathrm{C}$-terminal splice variants of $\mathrm{Ca}_{V} 1.3$ were identified, giving rise to long and short $\mathrm{Ca}_{V} 1.3$ isoforms (Bock et al., 2011; Shen et al., 2006; Singh et al., 2008). Long $\mathrm{Ca}_{V} 1.3$ channel isoforms were suggested to exhibit an intrinsic back-folding mechanism of the distal C-terminus interacting with the proximal $\mathrm{C}$-terminus to prevent fast $\mathrm{Ca}^{2+}$ dependent inactivation. Moreover, short isoforms exhibit hyperpolarized voltage-dependence of activation (Bock et al., 2011).

\subsubsection{The $\mathrm{Ca}_{V} \beta$ subunit}

$\mathrm{Ca}_{V} \beta$ subunits are encoded by four genes $\left(\mathrm{Ca}_{V} \beta_{1-4}\right)$ and crucial for VGCC trafficking and function (reviewed in (Buraei and Yang, 2010)). Studies of the molecular architecture of $\mathrm{Ca}_{V} \beta$ identified distinct regions within $\mathrm{Ca}_{V} \beta$, the core region of $\mathrm{Ca}_{V} \beta$ contains the so called HOOK domain, flanked by a SRC homology 3 (SH3) and a guanylate kinase domain (GK). Both of the latter domains are common protein interaction modules. The $\mathrm{Ca}_{V} \beta$ core region determines most of the $\mathrm{Ca}_{V} \beta$ functionality as VGCC subunit. $\mathrm{Ca}_{V} \beta$ subunits are located at the cytoplasmic site and, as mentioned above interact with the AID of the I-II linker region of the $\mathrm{Ca}_{V} \alpha 1$ subunit. $\mathrm{Ca}_{V} \beta$ subunits exert multiple functions, such as: (i) enhancement of VGCC transport to the plasma membrane via the AID domain (Bichet et al., 2000; Gebhart et al., 2010), (ii) tightening of synaptic $\mathrm{Ca}^{2+}$-exocytosis coupling (Kiyonaka et al., 2007) and (iii) shaping of $\mathrm{Ca}^{2+}$ channel gating properties by enhancing voltage-dependent activation and inactivation ((Berrou et al., 2001; Van Petegem et al., 2008); Figure 1.5A).

In IHCs, Neef and colleagues identified $\mathrm{Ca}_{V} \beta_{2}$ as the essential isoform of $\mathrm{Ca}_{V} 1.3$ channels. $\mathrm{Ca}_{V} \beta_{2}$ knock-out mice were profoundly hearing impaired and exhibited cochlear amplification deficits. IHC Ca ${ }^{2+}$ currents were decreased as a consequence of a reduced number of $\mathrm{Ca}_{V} 1.3$ at the plasma membrane with unchanged voltage-dependence of activation. Moreover, they also identified altered $\mathrm{Ca}^{2+}$-dependent inactivation, due to an accelerated slow inactivation component (Neef et al., 2009). Apparently, also $\mathrm{Ca}_{V} \beta_{3}$ and $\mathrm{Ca}_{V} \beta_{4}$ are expressed in IHC but only a $\mathrm{Ca}_{V} \beta_{4}$ mutation affected $\mathrm{Ca}_{V} 1.3$ expression at the plasma membrane (Kuhn et al., 2009). 


\subsubsection{The $\mathrm{Ca}_{V} \alpha_{2} \delta$ subunit}

$\mathrm{Ca}_{V} \alpha_{2} \delta$ subunits are members of the glycosylphosphatidylinositol (GPI)-anchored family. They consist of two separately translated proteins, encoded by the same gene. The $\mathrm{Ca}_{V} \alpha_{2}$ and $\mathrm{Ca}_{V} \delta$ proteins are co-translationally linked by disulfide bonds resulting in one subunit. $\mathrm{Ca}_{V} \delta$, an integral membrane protein forms the GPI-anchored $\mathrm{N}$-terminal and $\mathrm{Ca}_{V} \alpha_{2}$ the extracellular C-terminal region (Davies et al., 2010). To date four genes encoding for $\mathrm{Ca}_{V} \alpha_{2} \delta$ were identified, contributing to tissue specific $\mathrm{Ca}_{V} \alpha_{2} \delta$-1-4 expression throughout the central and peripheral nervous system, the brain, muscle tissue and the retina. The main function of $\mathrm{Ca}_{V} \alpha_{2} \delta$ subunits is an increased $\mathrm{Ca}^{2+}$ channel expression at the plasma membrane. The effect on VGCC expression ranges from a 2-fold increase of $\mathrm{Ca}_{V} 1.2$ in the presence of $\mathrm{Ca}_{V} \alpha_{2} \delta$-2 to 7-fold increase for $\mathrm{Ca}_{V} 2.1$ coexpressed with $\mathrm{Ca}_{V} \alpha_{2} \delta$-4 (reviewed in (Dolphin, 2013)). The $\mathrm{Ca}_{V} \alpha_{2} \delta$ dependent increase in VGCC abundance was shown to result from two mechanisms: (i) a reduced VGCC turnover and therefore, a higher $\mathrm{Ca}^{2+}$ channel stability in the membrane and (ii) a chaperone-like function of $\mathrm{Ca}_{V} \alpha_{2} \delta$ (Bernstein and Jones, 2007; Gao et al., 2000; Hoppa et al., 2012).

More recently, an extracellular metal ion-dependent adhesion site (MIDAS) in $\mathrm{Ca}_{V} \alpha_{2} \delta$ was identified to regulate VGCC function by tightening $\mathrm{Ca}^{2+}$ influx-exocytosis coupling. An intact MIDAS site induced an $80 \%$ increase in vesicle release probability, which for the first time implicated $\mathrm{Ca}_{V} \alpha_{2} \delta$ subunits as modulators of synaptic transmission (Hoppa et al., 2012). The investigation of a mutant mouse line (ducky mice) that lack the expression of full length $\mathrm{Ca}_{V} \alpha_{2} \delta$-2 due to a spontaneous mutation in the $C A C N A 2 D 1$ gene, showed that $\mathrm{Ca}_{V} \alpha_{2} \delta-2$ was the predominant subunit of $\mathrm{Ca}_{V} 1.3$ in IHC. These mice exhibited mild hearing impairment and affected cochlea amplification, with decreased $\mathrm{Ca}^{2+}$ currents and voltage sensitivity in IHCs (Fell et al., under revision).

\subsubsection{The $\mathrm{Ca}_{V} \gamma$ subunit}

The $\mathrm{Ca}_{V} \gamma$ subunit has four transmembrane segments with intracellular $\mathrm{C}$ - and $\mathrm{N}$ - termini. The biological function of the $\mathrm{Ca}_{V} \gamma$ is not restricted to VGCC modulation as suggested for $\mathrm{Ca}_{V} \beta$ and $\mathrm{Ca}_{V} \alpha_{2} \delta$ subunits, but in addition also regulates AMPA receptor trafficking, localization and modulation (Nicoll et al., 2006). The role of $\mathrm{Ca}_{V} \gamma$ as a VGCC subunit is controversial, due to a rare detection at VGCC. From channel isolation experiments, $\mathrm{Ca}_{V} \gamma$ was detected as $\mathrm{Ca}^{2+}$ channel subunit in skeletal muscles (Kuniyasu et al., 1992), but was mostly lacking in immunoprecipitation experiments of L-type $\mathrm{Ca}^{2+}$ channels in the brain (Ahlijanian et al., 1990). The function of the $\mathrm{Ca}_{V} \gamma$ subunit is dependent on respective subunit composition, the most consistent effect of VGCC modulation by $\mathrm{Ca}_{V} \gamma$ is the induction of a positive shift of voltage-dependence of activation (reviewed in (Black, 2003)). 


\subsubsection{Structure and function of $\mathrm{Ca}^{2+}$ binding proteins (CaBPs)}

$\mathrm{Ca}^{2+}$ binding proteins $(\mathrm{CaBPs})$ are members of the EF-hand $\mathrm{Ca}^{2+}$ binding proteins family, protein homologous to calmodulin (CaM). They are encoded by eight genes (Cabp1-8; Cabp3 however, is most probably a pseudogene) and are subdivided into two subfamilies with distinct intracellular localization profiles (Haeseleer et al., 2000). CaBP7 and CaBP8 are provided with a transmembrane domain and are predicted tail-anchored proteins with a predominant expression in synaptic vesicles (reviewed in (Haynes et al., 2012)). All CaBPs have in common that they are comprised of two lobes, each bearing a pair of EF-hands connected by an inter-lobe flexible $\alpha$-helix linker region. In contrast to $\mathrm{CaM}, \mathrm{EF}-\mathrm{h}$ and 2 in the $\mathrm{CaBP} \mathrm{N}$-lobe is not binding $\mathrm{Ca}^{2+}$. Moreover, the flexible helix linker region is prolonged by one helical turn, which was shown to increase the flexibility of the protein (Findeisen and Minor, 2010; Haeseleer et al., 2000). Two of the CaBPs, namely $\mathrm{CaBP} 1$ (except the caldendrin isoform) and $\mathrm{CaBP} 2$ are myristoylated at the $\mathrm{N}$-terminus, a post-translational modification that was suggested to enhance their plasma membrane stabilization.

While the $\mathrm{C}$-lobe of $\mathrm{CaBP} 1$ displays a $\mathrm{K}_{d, C a}$ of $2.5 \mu \mathrm{M}$, which is comparable to the $\mathrm{Ca}^{2+}$ binding kinetics of the $\mathrm{C}$-lobe of $\mathrm{CaM}$, the $\mathrm{N}$-lobe shows modest $\mathrm{Ca}^{2+}$ sensitivity due to a low $\mathrm{Ca}^{2+}$ affinity of EF-hand 1 ( $\mathrm{K}_{d, C a}>100 \mu \mathrm{M}$, (Wingard et al., 2005)). In contrast to ubiquitous CaM expression in all eukaryotic cells, CaBPs are restricted to sensory cells and neurons (Haeseleer et al., 2000). A very consistent function among CaBP1-5 is the regulation of VGCC by preventing $\mathrm{Ca}^{2+}$ dependent inactivation (Cui et al., 2007; Schrauwen et al., 2012; Yang et al., 2006). Amongst $\mathrm{CaBPs}$, CaBP1 is the most investigated isoform in respect to VGCC function: CaBP1 positively regulates $\mathrm{Ca}^{2+}$-dependent facilitation of $\mathrm{Ca}_{V} 1.2$ channels (Zhou et al., 2004) and enhances voltage-dependent inactivation in $\mathrm{Ca}_{V} 1.2 \mathrm{Ca}^{2+}$ channels (Oz et al., 2011). CaBP1 additionally modulates inositol 1,4,5 triphosphate receptors and, thereby, suppresses $\mathrm{Ca}^{2+}$ induced $\mathrm{Ca}^{2+}$ release (Kasri et al., 2004). Furthermore, CaBP1 interacts with transient receptor potential canonical type 5 channels (Kinoshita-Kawada et al., 2005) and myosin 1c (Tang et al., 2007).

To date only CaBP4 was reported to be required for normal vision, due to the discovery of a human mutation in the $C A B P 4$ gene that induced incomplete congenital stationary night blindness2 (Zeitz et al., 2006). Indeed, CaBP4 was localized to photoreceptor synaptic terminals and, apparently, is involved in rod and cone synapse formation and function as indicated by rod and cone synaptic deficits in the absence of CaBP4. Anatomically CaBP4 knock-out mice exhibited a thinner outer plexiform layer, due to the impaired development of photoreceptor synaptic terminals (Haeseleer et al., 2004). In addition, CaBP5 is expressed in rod bipolar cells and some cone bipolar cell types, but is not required for vision, as demonstrated by normal scotopic and photopic electroretinograms (ERGs) (Rieke et al., 2008).

In IHCs, CaBP1-5 were detected by immunohistochemistry, but their respective function is 
under current investigation. In CaBP4 knock-out mice, hearing was intact, although on IHC levels $\mathrm{Ca}^{2+}$-dependent inactivation (CDI) was modestly increased (Cui et al., 2007). Recent evidence drew the attention to $\mathrm{CaBP} 2$ as main $\mathrm{CaBP}$ isoform required for hearing, due to the discovery of a human mutation in the $C A B P 2$ gene that induces moderate-to-severe hearing impairment (Tabatabaiefar et al., 2011). This mutation likely causes the expression of a CaBP2 variant lacking the $\mathrm{C}$-terminal lobe and hence the primary $\mathrm{Ca}^{2+}$ sensing unit, resulting in impaired inhibition of $\mathrm{CDI}$ and reduced $\mathrm{Ca}^{2+}$ current densities in heterologous expression system (Schrauwen et al., 2012).

\subsubsection{Negative feedback regulation of $\mathrm{Ca}^{2+}$ channels}

\subsubsection{Voltage-dependent inactivation (VDI)}

VDI arises from the ability of VGCC to inactivate in response to voltage-dependent conformational changes. It is well accepted that structural components of VGCC that are responsible for VDI, are dependent on interactions between the pore of the VGCC (S6 segment) and the AID region of the cytosolic I-II loop of $\mathrm{Ca}_{V} \alpha 1$ (Dafi et al., 2004; Stotz and Zamponi, 2001) . The VDI response can be interpreted as a hinged-lid response of the AID-Ca $\beta$ complex to clog the channel pore (Stotz et al., 2000; Tadross et al., 2010). Dependent on the respective $\mathrm{Ca}_{V} \alpha 1$ and $\mathrm{Ca}_{V} \beta$ subunits, the extent of VDI can vary distinctly. Especially $\mathrm{Ca}_{V} \beta_{2 A}$ prevents VDI probably due to its palmitoylation-mediated anchoring to the plasma membrane (Restituito et al., 2000). This feature restricts AID mobility and therefore diminishes VDI. Remarkably, $\mathrm{Ca}_{V} 1.3$ channels exhibit an additional shield region within the S6 segment and are therefore more resistant to VDI (Tadross et al., 2010). This unique trait, so far only found in $\mathrm{Ca}_{V} 1.3$ channels, provides these channels with greater resistance to VDI.

\subsubsection{2 $\mathrm{Ca}^{2+}$-dependent inactivation (CDI)}

In addition to VDI, VGCC inactivate upon $\mathrm{Ca}^{2+}$ entry (i.e. $\mathrm{Ca}^{2+}$-dependent inactivation; CDI). CDI limits excessive $\mathrm{Ca}^{2+}$ influx in response to electrical activity and can be intrinsically regulated by the distal $\mathrm{C}$-terminal cytoplasmic tail of the $\mathrm{Ca}_{V} \alpha 1$ subunit itself. To antagonize CDI, the interaction between the proximal C-terminal regulatory domain (PCRD) and the distal C-terminal regulatory domain (DCRD) is crucial to decelerate inactivation (Bock et al., 2011). CDI of VGCC is induced by calmodulin $(\mathrm{CaM})$, an $\mathrm{EF}-$ hand $\mathrm{Ca}^{2+}$ binding protein that functions as $\mathrm{Ca}^{2+}$ sensor of the channel (Pitt, 2007). In a $\mathrm{Ca}^{2+}$ unbound Apo/CaM state, Apo/CaM pre-associates with the Pre-isoleucin-glutamin motive (Pre-IQ) and binds to the IQ domain in $\mathrm{Ca} / \mathrm{CaM}$ state, resulting in VGCC inactivation (reviewed in (Ben-Johny and Yue, 2014)). Some VGCC-expressing cell types, such as IHCs or cardiac pacemaker cells are dependent on mechanisms counteracting CDI to maintain opening despite of sustained activation. Beside other structural features, the expression of $\mathrm{CaBPs}$ is one possibility to achieve this task. Multiple $\mathrm{CaBPs}$ antagonize $\mathrm{CDI}$ induction by $\mathrm{CaM}$ via competitive binding to the channel's IQ domain. Due to the advantage of CaBPs over CaM 
by preventing CDI despite similar $\mathrm{K}_{d}$-values, CaBPs can be assumed to reside in closer proximity to the IQ-domain. Multiple regions within $\mathrm{Ca}_{V} \alpha 1$ allow constitutive $\mathrm{CaBP}\left(\mathrm{Ca}^{2+}\right.$ unbound $\left.\mathrm{CaBP}\right)$ binding, for example the N-terminal tail of $\mathrm{Ca}_{V} \alpha 1$ (Zhou et al., 2005) or the IQ domain itself (Findeisen et al., 2013). But whether the competition between CaBPs and CaM for the IQ domain is solely $\mathrm{Ca}^{2+}$-dependent requires further investigation.

\subsection{Genetic factors inducing sensorineural hearing impairment}

Hearing impairment is the most common sensory deficit and affects 360 million people world-wide (WHO). The underlying causes can vary from environmental factors such as infections, ototoxic drugs and noise trauma to genetic origins, which account for up to $50 \%$ of hearing impairment in newborns. Hearing can be impaired by dysfunction of outer and middle ear. (conductive hearing loss). Sensorineural hearing loss, the most common form of hearing impairment on the other hand, is characterized by the inability to either (i) convert sound into electrical signals or (ii) transmit the encoded signals to the brain. Genetic factors causing sensorineural hearing impairment are very heterogeneous and are affecting a variety of protein functions ranging from transcription factors, cytoskeletal proteins and components of the extracellular matrix to proteins interfering with vesicle morphology and ion channel gating (Smith et al., 1999).

\subsubsection{Impairment of the $\mathrm{K}^{+}$cycle}

Mutations interfering with the cellular $\mathrm{K}^{+}$cycle are abundant causes of hereditary hearing impairment and can be attributed to $50 \%$ of prelingual recessive deafness. Here, one of the most common disorders is caused by mutations in the GJB2 gene affecting Connexin 26. Connexin 26 is a gap junction protein expressed in a range of cell types within the organ of Corti and the stria vascularis. Mutations in Connexin 26 are compromising the ability to form functional connexons thus most likely affecting the $\mathrm{K}^{+}$recycling from the organ of Corti which results in reduced endocochlear potential.

\subsubsection{Amplification deficits}

Individuals who are affected by amplification deficits display reduced acoustic sensitivity and an abnormal loudness gain. For example, mutations in the TECTA gene, encoding for $\alpha$ Tectorin are affecting the structural integrity of the tectorial membrane, which affects amplification (DFNA8 and DFNB12; (Cremers et al., 2007)). Alternatively direct impairment of OHC function such as mutations in the gene SLC26A5 encoding for prestin impair OHC electromotility (Toth et al., 2007). Another prominent example are mutations in KCNQ4, a voltage-gated mechanotransduction channel expressed at the base of OHCs. KCNQ4 mutations affect the exit of $\mathrm{K}^{+}$ions coming in as the $\mathrm{OHC}$ transduction current, leading to impaired amplification and slow $\mathrm{OHC}$ degeneration (Coucke et al., 1999; Kharkovets et al., 2006; Kubisch et al., 1999). 


\subsubsection{Synaptopathies}

Hearing disorders caused by IHC synaptic malfunction are termed auditory synaptopathies (sometimes also subsumed under the broader term: auditory neuropathies). To date, multiple deafness genes are known to induce auditory synaptopathy. For example a mutation in the SLC17A8 gene encoding for Vglut3 (DFNA25, (Ruel et al., 2008)), induces impaired glutamate loading of synaptic vesicles, which hence fail to release neurotransmitter molecules. Moreover, alterations of presynaptic ultrastructure have been reported in these animals (Seal et al., 2008). Another example for IHC-specific synaptopathies are mutations in the multi-C2-domain protein otoferlin (DFNB9, (Yasunaga et al., 1999)). Otoferlin is thought to exert multiple functions in IHC ribbon synapse exocytosis, ranging from $\mathrm{Ca}^{2+}$ sensing to priming of synaptic vesicles and its absence results in almost complete loss of depolarization-dependent vesicle fusion.

A subclass of synaptopathies are "channelopathies" and characterized by mutations that directly involve ion channel functions. In IHCs, a human loss-of-function mutation was identified in the CACNA1D gene encoding for $\mathrm{Ca}_{V} 1.3 \mathrm{Ca}^{2+}$ channels with a mutation in the $\mathrm{S} 6$ segment of the $\mathrm{Ca}_{V} 1.3 \alpha 1$ subunit (Baig et al., 2011). This mutation causes congenital sinoatrial node dysfunction and deafness in the affected individuals (SANDD syndrome). In a CACNA1D resembling knock-out mouse model, the loss of $\mathrm{Ca}_{V} 1.3$ produced a $90 \%$ reduction in $\mathrm{Ca}^{2+}$ current (Platzer et al., 2000) alongside a concomitant $90 \%$ reduction in exocytosis (Brandt et al., 2003).

\subsection{Viral gene therapy as a tool to restore hearing}

A variety of gene therapeutic approaches to the ear for combating both genetic and idiopathic hearing impairment have been subject to extensive research within the past decades (reviewed in (Geleoc and Holt, 2014)). Due to the subject of this work, I want to highlight two recent discoveries in the field of gene therapeutic treatment of hereditary hearing loss. The first one involves hearing restoration in Vglut3 knock-out mice (Akil et al., 2012), the second one aims at hearing restoration in mice mutant for transmembrane channel-like 1 (Tmc1; (Askew et al., 2015)). These preclinical studies could build on previous establishment of AAV-mediated gene transfer into IHCs (Bedrosian et al., 2006; Reisinger et al., 2011).

\subsubsection{Hearing restoration in Vglut3 knock-out mice}

Vglut3 is expressed in IHCs and mice lacking Vglut3 are profoundly deaf due to the lack of glutamate in the synaptic vesicles (Ruel et al., 2008; Seal et al., 2008). Akil and colleagues applied adeno-associated virus serotype 2/1 (AAV2/1) carrying Vglut3 cDNA via direct microinjection into the round window of p1-3 knock-out mice. Although not carrying an IHC-specific promoter (chicken $\beta$-actin promoter was used), Vglut3 expression was restricted to IHC and within two weeks after virus delivery ABR thresholds were near-normal in $100 \%$ of tested animals up to 9 
months. Interestingly, with viral injections of p8-9 animals the longevity of hearing restoration was lost and restored ABR thresholds did not exceed 7 weeks. The reduced number of SGNs observed in knock-out mice (Seal et al., 2008) was not affected by the treatment, however, synapse morphology, more specifically ribbon shape and number of ribbon-tethered vesicles were partially restored. Whether this type of treatment is applicable for the respective human mutation described in DFNA25 is rather doubtful, due to the dominant character of this mutation and the uncommon appearance. Nevertheless, with this study, did Akil et al. demonstrated the potential of hearing restoration by AAV2/1-mediated gene delivery.

\subsubsection{Improved hearing in Tmc1 mutant mice}

The second study of this kind was undertaken by Askew and colleagues, who used AAV-mediated gene transfer to restore hearing in mice lacking trans membrane channel-like 1 (Tmc1). Tmc proteins have been implicated in the mechanotransduction apparatus and are affecting IHC and OHC function (Kawashima et al., 2011; Pan et al., 2013). Unlike Vglut3 mutations, human Tmc1 mutations are more abundant and can account for 4-8\% of genetic deafness in some populations (Pakistan, Iran). To date, 40 mutations have been identified most of which are of recessive nature (Kitajiri et al., 2007; Sirmaci et al., 2009). After screening through multiple AAV2/1 sterotypes $(1,2,6,8,9)$, AAV2/1 was confirmed to be the most suitable vehicle (Bedrosian et al., 2006), with transduction rates of $65 \%$ of IHCs and approximately $5 \%$ of OHCs. Individual IHC and OHC physiology remained unaffected in transduced cells, as exemplified by normal IHC transduction channel amplitude and mechanosensitivity. On a systems level, the restoration of ABR thresholds was not as pronounced as in the Vglut3 rescue approach; nevertheless, the previously absent ABR wave I was partially restored after AAV2/1-Tmc1 delivery, in particular in the low-to-mid frequency region. So far, virus-mediated gene therapy approaches to treat deafness are still in the early stages with additional challenges to overcome: both studies provide a prove of concept that validates the system as viable future approach and outline further questions that need to be addressed. These include the longevity of viral expression after transduction, the time window for application and cell- type specific targeting (i.e. IHC, OHC, SGN, etc.) amongst others.

\subsection{Aim of this project}

The aim of this study was to elucidate the role of CABP2 in the regulation of hair cell $\mathrm{Ca}^{2+}$ influx and synaptic sound encoding as well as to unravel the mechanism(s) underlying the hearing impairment DFNB93. Despite of the discovery of CaBPs in the late 1990s, to date very little is known about their function. Over the past years, $\mathrm{CaBPs}$ shifted into the focus of research due to their potent competition with $\mathrm{CaM}$ in the regulation of $\mathrm{Ca}^{2+}$ channels abolishing CaM-mediated CDI. However, so far, most studies focused on CaBP1, CaBP4 and CaBP5. Aside from structural predictions from gene sequence analysis and preliminary expression profiling, little is known 
about $\mathrm{CaBP} 2$. To address the cellular function of $\mathrm{CaBP} 2$, I chose two distinct approaches:

1. I performed patch-clamp recordings in a heterologous expression system to study the modulation of $\mathrm{Ca}_{V} 1.3$ by CABP2 and two clinically relevant mutant forms of CABP2, affecting the C- or N-lobes, respectively. With these to mutants, I aimed to identify lobe-specific functions of CABP2.

2. A Cabp2 deletion mutant mouse line was generated within this study which allowed me to test the requirement of Cabp2 for hearing and to identify the functional role of Cabp2 in IHC Ca ${ }^{2+}$ influx and sound encoding at the first afferent synapse in the auditory pathway. 
Introduction 


\section{Materials and Methods}

\subsection{Cloning of pEGFP-N1-hCABP2}

To obtain a C-terminally EGFP-tagged human CABP2 construct, CABP2 was amplified via PCR from pEGFP-C1-hCABP2 (RefSeq: NM_016366.2; modified sequence from cDNA clone IRAKp96K1413Q (ImaGees GmbH)), digested with the restriction enzymes NheI and BamHI and then ligated into a pEGFP-N1 backbone.

\section{PCR amplification:}

The PCR mix had the following composition (total volume of $50 \mu \mathrm{l}$ ): $2 \mathrm{mM}$ dNTPs, $5 \mu 1 \mathrm{Pfu}$ buffer, 50 pmol forward primer (NheI fwd; Appendix Table 8.1), 50 pmol reverse primer (CABP2wt or -EF1*: BamHI rev; $\mathrm{EF}^{\Delta 3 / 4}$ : BamHI rev1; Appendix Table 8.1), 100 ng DNA template and $1 \mu 1$ Pfu polymerase (for primer sequences see Appendix Table 8.1). The PCR reaction consisted of the following cycles: initial denaturation $\left(5 \mathrm{~min}, 95^{\circ} \mathrm{C}, 1 \mathrm{cycle}\right)$; denaturation $\left(30 \mathrm{~s}, 95^{\circ} \mathrm{C}\right), 30$ cycles of primer annealing $\left(30 \mathrm{~s}, 65^{\circ} \mathrm{C}\right)$, extension $\left(2 \mathrm{~min}, 72^{\circ} \mathrm{C}\right)$; and final extension $(10 \mathrm{~min}$, $72^{\circ} \mathrm{C}, 1$ cycle). The PCR product was purified with a PCR purification kit (NucleoBond DNA purification kit, Macherey-Nagel).

\section{Restriction digest and ligation of the hCABP2 insert and pEGFP-N1 backbone:}

Whole PCR products of purified wt or mutant CABP2 and $1 \mu \mathrm{g}$ of pEGFP-N1 DNA were digested with the restriction enzymes NheI and BamH1 in Nhe-buffer 2 with extra bovine serum albumin (enzymes and buffers: Life Technology) for $1 \mathrm{~h}$ at $37^{\circ} \mathrm{C}$. The restriction reaction was confirmed by agarose gel electrophoresis (110 V, $45 \mathrm{~min}$ ) and DNA (DNA-band length wt/CABP2-EF1*: 681 bp, CABP2-EF ${ }^{\Delta 3 / 4}: 513$ bp, pEGFP-N1: $4.7 \mathrm{~kb}$ ) was isolated by gel extraction (QuickGelExtraction Kit, Thermo Scientific). For the ligation reaction insert and backbone were mixed in a ratio of 3:1 and ligated with a fast T4 ligase in quick ligation buffer for $5 \mathrm{~min}$ at room temperature (RT) (Fast T4 DNA ligase, Thermo Scientific). Ligation mix was added to $50 \mu 1$ thermo-competent E.coli and kept on ice for $15 \mathrm{~min}$. The whole mix was then plated on kanamycin containing agar plates $(25 \mu \mathrm{g} / \mathrm{ml})$ and incubated over night at $37^{\circ} \mathrm{C}$.

\section{Identification of correct clones:}

A colony PCR was performed to identify CABP2 positive colonies. Therefore, a kanamycin containing agar plate was subdivided into 10 areas, then colonies were picked, transferred to the fresh plate and then lysed in $\mathrm{H}_{2} \mathrm{O}$ for PCR. Finally, PCR verified CABP2 positive clones were amplified and plasmid isolated with a Mini DNA purification kit (peqGOLD Plasmid Miniprep Kit, Peqlab) and correct sequences confirmed by sequencing. 


\subsection{Generation of stable HEK293/hSK3-1 cells}

The stable HEK293/hSK3-1 cells were provided by Dr. Luis Pardo (MPI for Experimental Medicine (MPI-EM), Göttingen). To generate HEK293 cells expressing the human small-conductance $\mathrm{Ca}^{2+}$ activated $\mathrm{K}^{+}$channel (hSK3-1), 5 wells of HEK 293 cells (293 Cell Line human, Sigma-Aldrich) were transfected with $3 \mu \mathrm{g}$ hSK3-1-DNA by lipofectamine (Lipofectamine 2000, Thermo Scientific) according to the manufacturers protocol. The culture medium was exchanged after 1 day with G418 containing culture medium with increasing geneticin (G418, Thermo Scientific) concentrations: $150-250-350-500-800 \mu \mathrm{g} / \mathrm{ml}$ per well (6-well plate). Antibiotic selection was performed for five days at $37^{\circ} \mathrm{C}$ in humidified atmosphere with $5 \% \mathrm{CO}_{2}$ saturation. After splitting mainly SK3-1 positive HEK293 cells survived due to the lack of antibiotic resistance in SK3-1 negative cells. To reduce the number of false positive HEK293/SK3-1 cells, surviving cells of the culture dish with highest G418 concentration but low cytotoxicity were maintained in culture medium containing $400 \mu \mathrm{g} / \mathrm{ml}$ of $\mathrm{G} 418$.

\subsection{Electrophysiological recordings of transiently transfected HEK293 cells}

HEK293/hSK3-1 cells were maintained in Dulbecco's modified Eagle medium/F12 Glutamax (Thermo Scientific), containing $10 \%$ FBS and $400 \mu \mathrm{g} / \mathrm{ml} \mathrm{G} 418$ at $37^{\circ} \mathrm{C}$ in humidified atmosphere with $5 \% \mathrm{CO}_{2}$. The HEK293/hSK3-1 cells were transfected at $<40 \%$ confluence with the transfection reagent ExGen 500 (Biomol), a protonated polyethylenimine according to the manufacturers protocol for $10 \mathrm{~mm}$ petri dishes. The total amount of $2 \mu \mathrm{g}$ DNA contained four subunits of the $\mathrm{Ca}_{V} 1.3 \mathrm{Ca}^{2+}$ channel: i) rat-Ca $\mathrm{Ca}_{V} 1.3_{42-A 2123 V}$ (GenBank accession number: AF370010), rat- $\beta_{2 a}$ (GenBank accession number: NM053851) and human- $\alpha_{2} \delta 1$ (GenBank accession number: $\mathrm{BC} 117468$ ) or for recordings with the corrected $\mathrm{Ca}_{V} 1.3$ clone ii) rat-Ca $\mathrm{Ca}_{V} 1.3 \alpha 1$ (Tan et al.,2011), rat- $\beta_{2 a}$ (GenBank accession number: NM053851), $\alpha_{2} \delta 1$ (GenBank accession number: NM_012919) and 50 ng of human CABP2 (RefSeq NM_016366.2). For transfections with $\mathrm{CABP} 2-\mathrm{EF}^{\Delta 3 / 4}$ only $5 \mathrm{ng}$ were transfected due to a higher expression level of this construct compared to CABP2-wt (Schrauwen et al., 2012). All patch clamp recordings were performed at RT 36-60 $\mathrm{h}$ after transfection.

The external solution was of the following composition (mM): $150 \mathrm{CholineCl}, 1 \mathrm{MgCl}_{2}, 10$ HEPES, $10 \mathrm{CaCl}_{2}, 100 \mathrm{nM}$ Apamin; pH 7.4 (adjustment with methanesulfonic acid) and 300$310 \mathrm{mosmol} / \mathrm{kg}$ (osmolality adjusted with CholineCl). For recordings of $\mathrm{I}_{B a}, 10 \mathrm{mM} \mathrm{CaCl}_{2}$ were replaced by $10 \mathrm{mM} \mathrm{BaCl}_{2}$. The internal solution contained the following (mM): $140 \mathrm{~N}$-methylD-glucamine (NMDG), 5 EGTA, $10 \mathrm{NaCl}, 1 \mathrm{MgCl}_{2}, 10$ HEPES, $2 \mathrm{MgATP}$; $\mathrm{pH} 7.4$ (adjusted with $\mathrm{NaOH}$ ) and $290 \mathrm{mosmol} / \mathrm{kg}$ (osmolality adjusted with NMDG).

The main components of the patch clamp setup were an inverse microscope (TE2000, Nikon), 
Generation of the $C a b p 2^{L a c Z / L a c Z}$ mouse model

a micromanipulator (Shutter Instruments), and an EPC-9 amplifier controlled by "Patchmaster" software (HEKA Elektronik). The pipette resistance was in the range of $3.5-8 \mathrm{M} \Omega$. For all recordings the voltage-clamp mode was used with $\mathrm{R}_{S}$ of $\leq 17 \mathrm{M} \Omega$ and $\mathrm{R}_{S}$ compensation of 70 $\%$, the recorded currents were low-pass filtered at $5 \mathrm{kHz}$ and sampled at $50 \mathrm{kHz}$. To subtract leak currents and capacitance transients a P/n protocol was applied (10 repeats). All traces were corrected offline for liquid junction potentials, which was calculated with the "patchers-powertool" add-on (Max Planck Institute for Biophysical Chemistry (MPIbpc), Göttingen) in IgorPro software.

\subsection{Generation of the $\operatorname{Cabp} 2^{L a c Z / L a c Z}$ mouse model}

\subsubsection{Long-Range PCR (LR-PCR) of ES cell (embryonic stem cell) clone genomic DNA}

LR-PCR was performed by Dr. Aleksandra Ivanovic of the MPI-EM according to the LR-PCR protocol provided by Knockout Mouse Project (KOMP) repository. In short, the DNA was extracted from ES cells by using a DNeasy Tissue Kit 250 (Qiagen 69506). The ES-cell monolayer was washed with cold PBS $\left(4^{\circ} \mathrm{C}\right)$ and isolated with a rubber policeman and transferred to a fresh tube on ice. After proteinase $\mathrm{K}$ and ethanol treatment the DNA was isolated with a DNA Mini spin column, washed with washing buffer and was finally eluted.

The primer combinations and amplicon sizes used in this study are summarized in Table 8.2 in the appendix. The LR-PCR was performed with a SequelPrep long PCR kit (Life Technology)

Table 2.1: LR-PCR thermo-cycle conditions.

\begin{tabular}{lll}
\hline Temp $\left[{ }^{\circ} \mathbf{C}\right]$ & Duration & Repetition \\
\hline \hline 93 & $3 \mathrm{~min}$ & $1 \mathrm{x}$ \\
\hline $68-1 /$ cycle & $15 \mathrm{sec}$. & \\
93 & $30 \mathrm{sec}$. & $8 \mathrm{x}$ \\
68 & $9 \mathrm{~min}$. & \\
\hline 93 & $15 \mathrm{sec}$. & \\
60 & $30 \mathrm{sec}$. & $30 \mathrm{x}$ \\
68 & $9 \mathrm{~min}$. \\
& sec/cycle & \\
\hline 68 & $9 \mathrm{~min}$. & $1 \mathrm{x}$ \\
\hline 4 & hold \\
\hline
\end{tabular}

thermal conditions were taken from the manufacturers protocol (Table 2.1). The amplicon size was checked by agarose gel-electrophoresis and the DNA isolated with a gel extraction kit. The DNA samples were sent for sequencing (AGCT-Lab, MPI-EM, Göttingen) to verify the correct insertion sequence at the 3' and 5' target allele, LoxP and FRT sites. 


\subsubsection{Recombination strategy for $\operatorname{Cabp} 2^{L a c Z / L a c Z}$ mice}

For the generation of $\mathrm{Cabp} 2^{\mathrm{LacZ} / \mathrm{LacZ}}$ mice, embryonic stem cell clones with the knock-out first (promoter driven) Cabp2 $2^{\text {tm2a(KOMP)Mbp }}$ allele (KOMP Repository, Oakland, California, USA) containing an IRES:LacZ Cabp2 trapping cassette were first cultured and then injected into a C57 black $6 \mathrm{~N}(\mathrm{C} 57 \mathrm{Bl} / 6 \mathrm{~N})$ blastocyst resulting in C57Bl/6N chimeric mice by the Transgenic Mouse facility of the MPI-EM. The chimera were bred with C57B1/6N to confirm germ-line transduction of the IRES:Lacz Cabp2 trapping allele. After Cre-recombination with a EIIa-cre, exon 3,4 in addition to the human $\beta$ actin promotor driven neomycin sequences were excised resulting in a stable constitutive Cabp2 mutant mouse line (Cabp2 $2^{\text {LacZ/LacZ }}$, Figure 2.1A), that expresses the LacZ reporter after exon 2. Starting with generation 3 the homozygous Cabp ${ }^{\text {LacZ/LacZ }}$ animals were used for experiments and were back-crossed with C57BL/6N in parallel during the whole project.

An alternative breeding strategy was to generate a conditional knock-out line, by first allowing flipase recombination (rosa flip was used to generate global recombination and therefore functionality of $\mathrm{Cabp2}$ ) to remove the LacZ reporter and the neomycin resistance resulting in floxed exon 3 and 4. After cre-recombination by a Vglut3-GFP promoter driven cre-line, an IHC specific Cabp2 knock-out was generated (Figure 2.1B). All animal experiments complied with national animal care guidelines, approved by the University of Göttingen Board for Animal Welfare and the "Animal Welfare Office" of the state of Lower Saxony.

\subsection{Isolation of the organ of Corti}

Animals were decapitated, then the skulls hemisected at the midsagittal line. After removing the brain, the cochleae were separated from the temporal bone and the organs of Corti exposed by removing the boney capsule and stripping off the stria vascularis. Finally the organ of Corti was isolated by peeling of the tissue in line of the modiolus.

\subsection{Isolation of the retina}

After decapitation of the animal the eye-balls were removed and the eye-cups isolated in HEPES Hanks solution under red light. After the separation of the segment layer four incisions were made resulting in the cross-shaped retina. The tissue was placed on a nitrocellulose membrane and flattened by surface tension of the dissection solution.

\subsection{Protein isolation and Western Blotting}

\subsubsection{Solutions}

\section{Lysis buffer:}

The lysis buffer was of the following composition in mM: $400 \mathrm{NaCl}, 1 \mathrm{EDTA}, 10 \mathrm{TrisHCl}$ and 
A

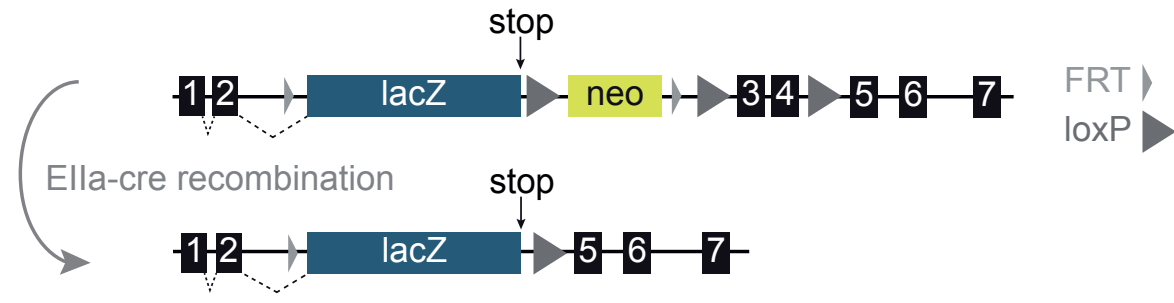

B
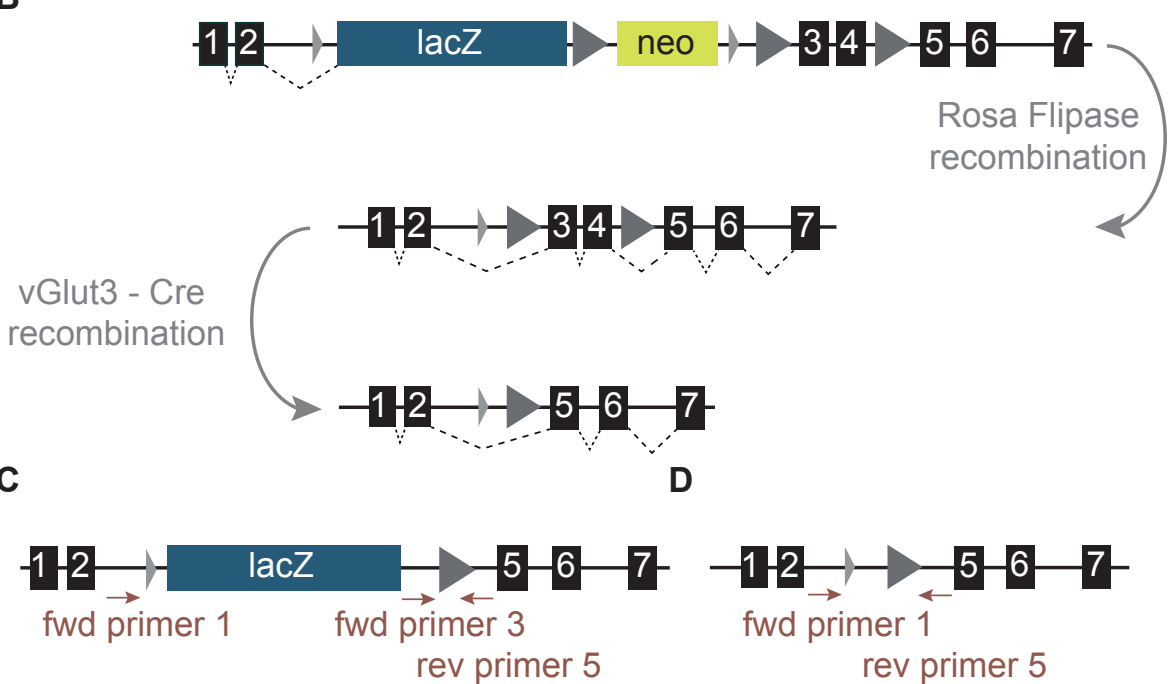

Figure 2.1: Knock-out strategy for the Cabp2 $2^{\text {LacZ/LacZ }}$ mouse line: (A) General scheme of the IRES:LacZ Cabp2 trapping allele strategy with LacZ reporter in blue, neomycin resistance for cassette positive ES cell clones in yellow and the FRT and loxP sites respectively are highlighted as gray triangles. IRES:Lacz Cabp2 trapping strategy after cre-recombination resulting in $C a b p 2^{L a c Z / L a c Z}$, a Cabp2 mutant line only expressing exon 1 and 2 with a $\beta$-galactosidase tag containing a stop codon. (B) Strategy to generate a conditional Cabp2 knock-out: After flipase recombination LacZ reporter and neomycin sequences are removed resulting in a floxed Cabp2 allele. After cre-recombination a conditional Cabp2 knock-out can be obtained. (D) Genotyping

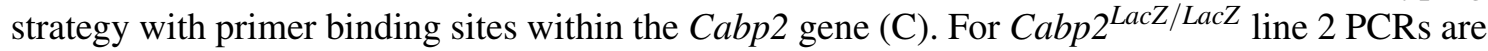
performed to identify the $1.7 \mathrm{~kb}$ band for wild-type and $360 \mathrm{bp}$ band for the mutant Cabp2 after cre-recombination. $\left(\mathrm{D}_{i}\right)$ For the conditional knock-out only one PCR is needed, flip-recombined genotypes can be identified by a $1.9 \mathrm{~kb}$ band for recombination and $1.7 \mathrm{~kb}$ for wt. 
$0.1 \%$ Triton. Protease inhibitor solution (cOmplete ULTRA Tablets, Roche) was prepared freshly before use: 2.5 pills in $5 \mathrm{ml} \mathrm{H}_{2} \mathrm{O}$ and added in a ratio of 5:1.

\section{Running buffer:}

The stock solution of the running buffer contained the following (in M): 0.25 Tris, 1.9 Glycine, 1 $\%$ SDS in $\mathrm{H}_{2} \mathrm{O}$. One day before use, the stock solution was diluted $1: 10$ in $\mathrm{H}_{2} \mathrm{O}$ containing $0.1 \%$ SDS as final concentration.

\section{Transfer buffer:}

The transfer buffer contained the following (in mM): 25 Tris, 192 Glycine and 20\% Methanol diluted in $\mathrm{H}_{2} \mathrm{O}$ at $\mathrm{pH} 8.3$.

\section{Tris buffered saline-Tween (TBS-T):}

TBS-T contained the following (in $\mathrm{mM}$ ): 20 Tris, $136.89 \mathrm{NaCl}$ and $0.1 \%$ Tween 20 diluted in $\mathrm{H}_{2} \mathrm{O}$ and adjusted to $\mathrm{pH}$ 7.6.

\section{Blocking solution:}

For blocking 5\% skimmed milk powder was dissolved in TBS-T.

\section{Ponceau-S staining solution:}

The Ponceau-S staining solution contained $0.2 \%$ Ponceau-S and 5\% acetic acid diluted in $\mathrm{H}_{2} \mathrm{O}$.

\subsubsection{Western Blotting Procedure}

To isolate the protein content of the apical turn of the Organ of Corti, six cochleae were processed as described in section 2.5 and the tissues were treated with $50 \mu 1$ lysis buffer. For that purpose, samples were collected on ice and subsequently lysed for $10 \mathrm{~min}$ prior to ultrasonication with a hand-sonicator at $25 \%$ power, for 3 cycles of $12 \mathrm{~s}$ each. After cell lysis, the protein solution was separated from cell membrane fragments by centrifugation $\left(1 \mathrm{~h}, 10000 \mathrm{rpm}, 4^{\circ} \mathrm{C}\right)$. The supernatant was transferred to a fresh tube and stored at $-80^{\circ} \mathrm{C}$ prior to the experiment.

To break disulfide bonds, protein samples were heated for $5 \mathrm{~min}$ at $95^{\circ} \mathrm{C}$ and then mixed with loading dye: $20 \mu \mathrm{l}$ protein mix (50 $\mu \mathrm{g}$ ) plus $5 \mu 1$ loading dye. Per pocket $7 \mu 1$ of PageRuler Plus prestained protein ladder (Life Technology, Darmstadt, Germany) or $25 \mu 1$ protein sample were added and the electrophoresis chamber was filled with running buffer. The electrophoresis started at $80 \mathrm{~V}$ for $20 \mathrm{~min}$ and subsequently ran at $100 \mathrm{~V}$ for additional $1-1.5 \mathrm{~h}$ dependent on the gel density. Subsequently, the SDS-polyacrylamide gel was blotted onto a nitrocellulose membrane in transfer configuration on ice for $3 \mathrm{~h}$ at $250 \mathrm{~mA}$. The nitrocellulose membrane was stained with Ponceau-S staining solution for $30 \mathrm{~s}$ to confirm successful protein transfer. Reactive amino groups were blocked with blocking solution for $1 \mathrm{~h}$ on a shaker. After blocking, the membrane 
was incubated with the respective primary antibody diluted in blocking solution over night at $4^{\circ}$ followed by 3 washing steps for $20 \mathrm{~min}$ at RT with TBS-T. The incubation with secondary antibody was kept for $1 \mathrm{~h}$ at RT with antibodies diluted in TBS followed by another washing step. The blot was developed for $60 \mathrm{~s}$ with a two component developing solution (Pierce ECL Western Blotting Substrate, Thermo Scientific) and imaged with a chemilumineszenz system (Intas ChemoCam Imager) running ChemoStar Imager software.

\subsection{Reverse transcription (RT) of Cabp2 mRNA}

To confirm Cabp2 absence in $C a b p 2^{\text {LacZ/LacZ }}$ animals, mRNA of six organs of Corti were isolated, reverse transcribed and the cDNA amplified by PCR.

The tissue was homogenized by adding $200 \mu 1$ of Trizol reagent (Invitrogen, Bremen, Germany) per organ of Corti and processed according to the manufacturers protocol. The mRNA pellet was resuspended in RNAse free $\mathrm{H}_{2} \mathrm{O}$ for $10 \mathrm{~min}$ at $55^{\circ} \mathrm{C}$. For reverse transcription GeneAmp RNA PCR kit (Invitrogen, Bremen, Germany) was employed and first $0.5 \mu 1$ oligo dt, $0.3 \mu 1$ random hexamers and $1 \mu \mathrm{l}(10 \mathrm{mM}) \mathrm{dNTPs}$ were added to $20 \mu \mathrm{l}$ of resuspended mRNA, kept for $5 \mathrm{~min}$ at $65^{\circ} \mathrm{C}$ before the reverse transcription mix consisting of $4 \mu \mathrm{l}$ first strand buffer, $0.1 \mathrm{M} \mathrm{DTT}, 1 \mu \mathrm{l}$ of RNAse out and $1 \mu 1$ SuperScript polymerase were added. For reverse transcription the samples were kept $10 \mathrm{~min}$ at RT, $30 \mathrm{~min}$ at $37^{\circ} \mathrm{C}, 60 \mathrm{~min}$ at $42^{\circ} \mathrm{C}$ and $5 \mathrm{~min}$ at $70^{\circ} \mathrm{C}$. Finally the cDNA was precipitated with $100 \% \mathrm{EtOH}$ and the cDNA content amplified by PCR with the following primer pairs: PCR 1: exon 1 fwd, exon 5 rev; PCR 2: LacZ fwd, exon 5 rev; PCR 3: glutaraldehyde dehydrogenase (GAPDH) fwd, GAPDH rev (see Appendix 8.1).

\subsection{LacZ staining of $\operatorname{Cabp} 2^{L a c Z / L a c Z}$ tissue}

\subsubsection{Solutions}

Whole mount fixative (mM):

$0.2 \%$ glutaraldehyde, 5 EGTA and $2 \mathrm{MgCl}_{2}$ in 100 PBS; $\mathrm{pH}$ 7.3.

Detergent rinse solution $(\mathrm{mM})$ :

$0.02 \%$ Igepal, $0.01 \%$ 7-Deoxycholic acid $\mathrm{Na}^{+}$salt and $2 \mathrm{MgCl}_{2}$ in $100 \mathrm{mM}$ PBS, pH 7.3.

X-Gal staining solution $(\mathrm{mM})$ :

$0.02 \%$ Igepal, $0.01 \%$ 7-Deoxycholic acid $\mathrm{Na}^{+}$salt, $5 \mathrm{FeK}_{2}(\mathrm{CN})_{6}, 5 \mathrm{FeK}_{3}(\mathrm{CN})_{6}$ and $2 \mathrm{MgCl}_{2}$ in 100 mM PBS; pH 7.3. 


\subsubsection{Procedure}

To identify Cabp 2 gene-expression, $\beta$-galactosidase activity was reported by $\mathrm{X}$-Gal stainings. The staining solution was stored in dark at RT. Prior to use, $1 \mathrm{mg} / \mathrm{ml} \mathrm{X- \beta}-\mathrm{Gal}$ in N,N-dimethylformamide (Carl Roth) was added. The cochlea was perfused with approximately $1 \mathrm{ml}$ of whole mount fixative through the round and oval window after cracking a hole into the apex of the cochlea bone. For fixation, the cochlea was kept on ice for $10 \mathrm{~min}$ and subsequently washed $3 \times 15$ min with detergent rinse solution. For staining, the cochlea was immersed in $1 \mathrm{mg} / \mathrm{ml} \mathrm{X}-\beta$-Gal containing staining solution and incubated in dark at $37^{\circ} \mathrm{C}$ over night. After removing the staining solution by washing 3 in PBS at room temperature the samples were imaged. For imaging the whole cochlea, the cochlea was treated with 2,2' Thioethanol to make the bone transparent (10 min).

For X-Gal stainings of the retina, the flattened tissue adhering on a nitrocellulose membrane was fixed for $20 \mathrm{~min}$ in whole mount fixative and processed subsequently according to the staining procedure of the cochlea.

\subsection{Immunohistochemistry}

\subsubsection{Solutions}

\section{Phosphate buffer (PB) $240 \mathrm{mM}$ :}

$27.52 \mathrm{~g} \mathrm{Na}_{2} \mathrm{HPO}_{4}, 5.52 \mathrm{~g} \mathrm{NaH}_{2} \mathrm{PO}_{4}$ in $11 \mathrm{H}_{2} \mathrm{O}$.

\section{Phosphate buffer saline (PBS):}

5 phosphate buffered saline tablets (Sigma-Aldrich) in $11 \mathrm{H}_{2} \mathrm{O}$; components (in g): $8 \mathrm{NaCl}, 0.2$ $\mathrm{KCl}, 1.15 \mathrm{Na}_{2} \mathrm{HPO}_{4}, 0.2 \mathrm{KH}_{2} \mathrm{PO}_{4}$.

PFA (paraformaldehyde) fixation solution:

$550 \mu 1$ of $37 \%$ PFA in PBS for a final concentration of $4 \%$ PFA.

\section{Wash buffer (in mM):}

$20 \mathrm{~PB}, 0.3 \%$ Triton $\mathrm{X}-100,450 \mathrm{NaCl}$ in $11 \mathrm{H}_{2} \mathrm{O}$.

Goat serum dilution buffer (GSDB) (mM):

$16.7 \%$ GSDB, $20 \mathrm{~PB}, 0.3 \%$ Triton $\mathrm{X}-100,450 \mathrm{NaCl}$ in $36.45 \mathrm{ml} \mathrm{H}_{2} \mathrm{O}$.

\subsubsection{Procedure for the organ of Corti}

The morphology of IHCs was visualized by using immunohistochemical stainings of the organ of Corti. For immunohistochemical stainings the cochlea was isolated and the bone at the apical turn of the cochlea was opened to enable fixative perfusion through the round and the oval window 
with $4 \%$ paraformaldehyde in PBS. Depending on the target protein, the fixation reaction was performed on ice for 15 or $60 \mathrm{~min}$. After fixation the organ of Corti was transferred into PBS and washed followed by the blocking of reactive amino groups by GSDB. The primary antibodies were diluted in GSDB at a concentration according to Table 2.2 for an incubation duration of 16 $\mathrm{h}$ at $4^{\circ} \mathrm{C}$. Subsequently, the primary antibody was removed by washing the samples with washing buffer before adding the secondary antibody (concentration according to Table 2.2) diluted in GSDB for $1 \mathrm{~h}$ at RT. After a final washing procedure in washing buffer and storage of the tissue in $5 \mathrm{mM}$ PB (5 min ), the samples were mounted on glass slides with Mowiol mounting medium (prepared according to the manufacturers protocol; Carl Roth) and stored at $4{ }^{\circ} \mathrm{C}$ until imaging (SP5, Leica). The images were acquired with a 63x oil immersion objective with an $488 \mathrm{~nm}$ Argon and 594 DPSS laser for excitation and a sequential scan of 4 repetitions with a step size of approximately $0.7 \mu \mathrm{m}$.

\subsubsection{Procedure for retinal slices}

For immunostainings of retinal slices the retina was fixed for $60 \mathrm{~min}$ in $4 \%$ paraformaldehyde at RT and subsequently washed 3 times in PBS and transferred into sucrose gradient solutions of $10 \%$ (10 min, RT), 20\% ( $2 \mathrm{~h}, \mathrm{RT}$ ) and $25 \%$ (over night, $4^{\circ} \mathrm{C}$ ). After embedding the retina in cryomatrix (Life Technology) the tissue was frozen in a cryostat $\left(-25^{\circ} \mathrm{C} ; 2800\right.$ Frigocut, Reichert-Jung), sliced (thickness: $60 \mu \mathrm{m}$ ) and stored at $-80^{\circ} \mathrm{C}$ until staining. The immunostaining procedure was the same as described for the organ of Corti.

Table 2.2: Implementation of immunostainings: Antibody host animals were chicken (ch), guinea pig (gp), mouse (m) and rabbit (r).

\begin{tabular}{lllll}
\hline Fixative & $\begin{array}{l}\text { Time } \\
(\text { min) }\end{array}$ & $\begin{array}{l}\text { Blocking } \\
\text { solution }\end{array}$ & $\begin{array}{l}\text { Primary } \\
\text { antibody }\end{array}$ & $\begin{array}{l}\text { Secondary } \\
\text { antibody }\end{array}$ \\
\hline \hline PFA 4\% & 15 & GSDB & $\begin{array}{l}\text { m-a-Ctbp2 } \\
\text { r-a-GluA2/3 }\end{array}$ & $\begin{array}{l}\text { g-a-m Alexa 488 } \\
\text { g-a-r Alexa 488 }\end{array}$ \\
& & & gp-a-Parvalbumin & g-a-gp Alexa 405 \\
PFA 4\% & 60 & GSDB & m-a-calbindin D-28 K & $\begin{array}{l}\text { g-a-m Alexa 568 } \\
\text { PFA 4\% }\end{array}$ \\
& 60 & GSDB & ch-a- $\beta$-galactosidase \\
PFA 4\% & 60 & GSDB & $\begin{array}{l}\text { g-a-ch Alexa 488 } \\
\text { g-a-m Alexa }\end{array}$ \\
\hline
\end{tabular}




\subsection{Patch clamp recordings of inner hair cells}

\subsubsection{Solutions}

\section{HEPES Hanks dissection solution ( $\mathrm{mM})$ :}

$5.36 \mathrm{KCl}, 141.7 \mathrm{NaCl}, 10 \mathrm{HEPES}, 0.5 \mathrm{MgSO}_{4} * 7 \mathrm{H}_{2} \mathrm{O}, 1 \mathrm{MgCl} * 6 \mathrm{H}_{2} \mathrm{O}$; pH 7.2, osmolality 285 $\mathrm{mOsm} / \mathrm{kg}$. Prior to use $16.93 \mathrm{mM}$ Glucose and $3.42 \mathrm{mM}$ L-Glutamine were added.

\section{Extracellular Ringer solution (mM):}

$2.8 \mathrm{KCl}, 113 \mathrm{NaCl}, 10 \mathrm{HEPES}, 1 \mathrm{CsCl}, 35 \mathrm{TEA}-\mathrm{Cl}, 1 \mathrm{MgCl}_{2}, 2 \mathrm{Ca}^{2+} / \mathrm{Ba}^{2+}{ }_{2} \mathrm{Cl}$; $\mathrm{pH}$ 7.2, osmolality $300 \mathrm{mOsm} / \mathrm{kg}$.

\section{Intracellular solution for ruptured patch $(\mathbf{m M})$ :}

130 Cs-Gluconate, 10 TEA-Cl 2 , 10 4-AP (4-aminopyridine; Merck), $1 \mathrm{MgCl}_{2}, 2 \mathrm{MgATP}, 0.3 \mathrm{Mg}$ GTP, 0.5 BAPTA, and 0.5 EGTA 10 HEPES; pH 7.2, osmolality $290 \mathrm{mOsm} / \mathrm{kg}$.

\section{Intracellular solution for perforated patch $(\mathbf{m M})$ :}

137 Cs-Gluconate, 10 TEA-Cl, 10 4-AP, $1 \mathrm{MgCl}_{2}, 10$ HEPES; pH 7.2, osmolality 184 mOsm/kg. Prior to use $250 \mu \mathrm{g} / \mathrm{ml}$ of amphotericin B freshly dissolved in DMSO (final concentration: $0.6 \%$ ).

\subsubsection{Procedure}

For patch clamp recordings two age groups of $C a b p 2^{\text {LacZ/LacZ }}$ mice were used, either at post natal day p15-19 or at p35-42. The organ of Corti was isolated in HEPES Hanks dissection solution. After dissection, the organs of Corti were transferred into the recording/bath chamber and fixed under a grid consisting of a U-piece made of stainless steel with superglued nylon strings. The IHC of the freshly dissected apical full coil were patch-clamped after removing first the tectorial membrane followed by multiple cell layers consisting of the Hensen's cells, OHC, outer and inner pillar cells and ultimately phalangeal cells. Patch pipettes were pulled with a horizontal P-97 filament puller (Shutter Instrument CO.) and pipette diameter fine-tuned by heat polishing for an inner diameter of approximately $1.8 \mu \mathrm{m}$. For all experiments, pipettes were coated with Sylgard (GE Bayer Silicones, BAYER). All electrophysiological experiments were performed with an EPC-9 amplifier (HEKA Elektronik) and controlled by "Patchmaster" software in voltage-clamp mode. Stray capacitance between input and electrode was compensated with the auto $\mathrm{C}_{F a s t}$ feature of the EPC-9, while whole cell capacitance compensation was adjusted with the auto $\mathrm{C}_{\text {Slow }}$ feature. The currents were low-pass filtered at $2.9 \mathrm{kHz}$ and sampled at $50 \mathrm{kHz}$. To subtract leak currents and uncompensated capacitance transients a $\mathrm{P} / \mathrm{n}$ protocol (10 repeats) was performed. Voltage traces were corrected offline for voltage-drops across series resistance $\left(\mathrm{R}_{S}\right)$ and for liquid junction potentials, which was calculated with the "patchers-power-tool" add-on (MPIbpc, Göttingen). 


\subsection{Extracellular recordings of spiral ganglion neurons}

SGN recordings were performed by Anna Gehrt. In short, mice of 5-8 weeks of age were anesthetized with a combination of urethane $(1.32 \mathrm{mg} / \mathrm{kg})$, xylazine $(5 \mathrm{mg} / \mathrm{kg})$, and buprenorphine $(0.1$ $\mathrm{mg} / \mathrm{kg}$ ) injected intraperitoneally. The mouse body temperature was maintained at $37^{\circ} \mathrm{C}$ with a rectal temperature-controlled heat bed. Post anesthesia, mice were tracheostomized and placed in a stereotactic apparatus for surgery. An opening was made in the left Occipital bone and the cerebellum was partially removed, to approach the SGN through the cochlear nucleus with a glass electrode filled with $3 \mathrm{M} \mathrm{NaCl}$ and $2 \%$ methylene blue. A constant noise burst stimulus of $80 \mathrm{~dB}$ was presented to detect SGN activity. Upon detection of single-unit firing, SGNs were identified by the electrode position ( $1200 \mu \mathrm{m}$ below the surface of cochlear nucleus), irregular firing pattern and their primary-like peristimulus time histogram (PSTH). Upon detection of a sound-responsive unit, spontaneous firing rate was determined in the absence of any sound stimulus. Then a tuning curve was obtained by varying the intensity and frequency of $15 \mathrm{~ms}$ tone bursts, to estimate the best threshold of hearing and the characteristic frequency (CF) with a 1/32 octave and $2 \mathrm{~dB}$ precision. Further characterization of single auditory units was done at $\mathrm{CF}$ and $30 \mathrm{~dB}$ above threshold.

\subsection{Systems physiology}

\subsubsection{Electroretinography (ERGs)}

ERGs were recorded according to previously published protocols (tom Dieck et al., 2012). The experiments were performed in a dark room with dimmed red light. In brief, adult mice (7-8 weeks) were darkadapted over night, then anesthetized by a mixture of ketamin and xylazine (125 $\mu \mathrm{g} / \mathrm{g}, 2.5 \mu \mathrm{g} / \mathrm{g}$ ) and positioned on the setup on a heat blanket to maintain the body temperature at $37^{\circ} \mathrm{C}$. To widen the pupils $1 \%$ atropin was applied to the left eye. The electrode configuration was the following: an $\mathrm{AgCl}$ electrode was positioned on the cornea, the reference electrode subcutaneously close to the rostrum tip; grounding was accomplished via an electrode inserted near the tail. White light flashes of increasing intensity 0.0002-0.15-0.21-0.25-0.28-0.292-0.298 $\mathrm{cd} * \mathrm{~s} / \mathrm{m}^{2}$ were repeated $10 \mathrm{x}$ and means calculated. The stimuli were presented for $0.1-1-2 \mathrm{~ms}$ with an inter-stimulus intervals of $5 \mathrm{~s}$. The recordings were amplified, bandpass-filtered (400-4000 Hz) and sampled at a rate of $24 \mathrm{kHz}$.

\subsubsection{Auditory brainstem responses (ABRs)}

Mice were anesthetized as described earlier (Section 2.13.1). The electrode configuration of three subcutaneous needles was the following: the active electrode was positioned underneath the pinna, the reference electrode on the vertex and the ground electrode near the tale. For ABR recordings and subsequent analysis a TDT II System controlled by BioSig software (Tucker Davis Technologies, Mathworks) was used. ABR were obtained in two repetitions of 1000 responses to tone bursts 
(10 ms plateau, $1 \mathrm{~ms} \cos ^{2}$ rise/fall; in dB SPL RMS) of increasing frequencies: 4-6-8-12-16-24-32 $\mathrm{kHz}$ presented at $40 \mathrm{~Hz}$ or clicks $(0.03 \mathrm{~ms}$ dp SPL peak equivalent) at $20 / 100 \mathrm{~Hz}$ in the free field ipsilaterally presented by JBL-2402 speaker (JBL GmbH\&Co). The recordings were amplified (50 $000 \mathrm{x}$ ), bandpass filtered $(400-4000 \mathrm{~Hz})$ and sampled at $50 \mathrm{kHz}$ for $20 \mathrm{~ms}$. ABR thresholds were obtained by visual inspection of two separate experimenters that independently determined the lowest stimulus intensity evoking reproducible response waveforms.

\subsubsection{Distortion product otoacoustic emissions (DPOAEs)}

DPOAEs were recorded by presenting continuous primary tones at frequency1 (f1:6-9.4-13.3$18.9 \mathrm{kHz}$ ) and frequency 2 in the following relation: $\mathrm{f} 2=1.2 * \mathrm{f} 1$. The respective sound intensities were the following: I2=I1-10dB. Tones were played through a MF1 speaker system (Tucker Davis Technologies), detected by a miniature probe containing a microphone (Sennheiser) and amplified. DPOAE amplitude defined as $\mathrm{A}=2 * \mathrm{f} 2$-f1 was derived after Fourier transformation by a routine written in Matlab (Mathworks).

\subsection{Viral rescue of $C a b p 2^{L a c Z / L a c Z}$}

\subsubsection{Cloning of AAV2-HBA-EWB-mCabp2}

The AAV backbone AAV-HBA-EWB was provided by Sebastian Kuegler (Viral Vectors Lab) and encoded AAV2/1 capsid proteins, a human $\beta$ actin promotor (HBA) combined with a human CMV enhancer, an EGFP reporter, a woodchuck post-transcriptional regulatory element (WPRE) and a human growth hormone (HGH). Mouse Cabp2 ( $m$ Cabp 2 ) transcription variant 1 sequence (NM013878) in pCMV6 backbone (OriGene Technologies) was amplified by PCR (40 cycles, $\mathrm{T}_{\text {annealing: }} 60^{\circ} \mathrm{C}$ ) by primer pair fwd AAV2, rev AAV2 (sequence see Appendix Table 8.1) and digested by restriction enzymes Nhe 1 and EcoR 1 for $1 \mathrm{~h}$ and $37^{\circ} \mathrm{C}$. After gel-extraction the DNA bands of mCabp2 (amplicon length: $666 \mathrm{bp}$ ) and AAV-HBA-EWB (amplicon length: $5.535 \mathrm{~kb}$ ) were ligated over night at $16^{\circ} \mathrm{C}$ with T4 DNA ligase. For the amplification of viral vectors electrocompetent cells for cloning DNA with secondary structures were used (SURE, Stratagene). The constructs were checked by control digest with EcoR1 and Nhe1 and sequenced (AGCT-Lab, MPI-EM, Göttingen).

\subsubsection{Embryonic transuterine otocyst injection of AAV2-HBA-EWB-mCabp2-EGFP}

Transuterine otocyst miroinjections were performed by Sangyong Jung as described previously (Bedrosian et al., 2006; Reisinger et al., 2011). In short, pregnant females were anesthetized by a mixture of $125 \mu \mathrm{g} / \mathrm{g}$ ketamine and $2.5 \mu \mathrm{g} / \mathrm{g}$ xylazine. A laparotomy was performed under a dissection microscope. The uterus was externalized and developing embryos were oriented to allow access to the otocyst of the left ear by exposing the anterior cardinal vein and the fourth 
ventricle as hallmark structures. Otocysts were approached with a glass needle, that was prepared prior to operation. The glass pipette had an inner diameter of $10-20 \mu \mathrm{m}$ and were filled with a mixture of AAV2-HBA-EWB-mCabp2-EGFP suspension and fast green FCF dye (titer final: 2.48 $\exp (12) \mathrm{GC} / \mathrm{ml}$, green fast dye $0.1 \%$ ) and oriented at an angle of $45^{\circ}$. They were connected to a pressure injector (PL1-100: Harvard Apparatus) and 2-3 shots of virus suspension were injected with 0.13-0.34 bar. After injections the uterus was returned to the peritoneal cavity and incisions were stitched. After surgery, dams recovered over night in a cage at $37^{\circ} \mathrm{C}$.

\subsection{Data analysis}

Cloning strategies were designed in GENtle (Magnus Maske), in addition test digests and alignments of sequenced DNA were performed in GENtle. Electrophysiological data was analysed in "Igor Pro 6" (Wavemetrics), single unit recordings with MatLab (The MathWorks). Immunostainings were adapted and analysed in ImageJ software (ImageJ, SciJave, (Schneider et al., 2012)) and figures were assembled in Adobe Illustrator software. Multiple recordings were presented as mean values \pm standard error of the mean (s.e.m.). In general two data sets were compared by Student's unpaired, two-tailed t-test when proven to be independent (test: Serial Randomness test), equally (test: Kolmogrov Smirnov test) and normally distributed (assessed by Jarque-Bera test) and with equal variances (test: $\mathrm{f}$-test). Statistically significant differences are indicated with $*$ for $\mathrm{p}$-values $<0.05, * * \mathrm{p}<0.01$ and $* * * \mathrm{p}<0.005$. Whenever the two data sets did not fulfill the requirements of the Student 't-test (see above) the nonparametric unpaired, two-tailed Wilcoxon Rank Sum test (Mann-Whitney test) was used.

\subsubsection{Current-Voltage curves (IV-curves)}

Steady-state current-voltage relationships (IVs) were obtained by plotting the average $\mathrm{I}_{C a}$ between 13-18 ms of a $20 \mathrm{~ms}$ step against the respective test potential ranging from -84 to $+71 \mathrm{mV}$ in $5 \mathrm{mV}$ increments, baseline subtracted for the mean of $2-5 \mathrm{~ms}$ prior to the depolarizing pulse. For HEK293/SK3-1 cell experiments the currents were normalized to the cell capacitance resulting in current densities. IVs were analysed by considering the reversal potential of $\mathrm{Ca}^{2+}$ or $\mathrm{Ba}^{2+}$ (assuming that no further channels open for higher depolarizations at these voltages such that any change in current is solely due to the change in electrical driving force) derived by line fits within the linear range of the respective IV curve ( -5 to $10 \mathrm{mV}$ for CABP2-wt; $5-15 \mathrm{mV}$ for $\mathrm{Ca}_{V} 1.3_{42-A 2123 V}$ dependent on shifts of voltage-dependence):

$$
I_{C a, B a}=G *\left(V-V_{r e v}\right)
$$

$\mathrm{I}_{C a, B a}$ represents the respective $\mathrm{Ca}^{2+}$ or $\mathrm{Ba}^{2+}$ current, $\mathrm{g}$ membrane conductance of the cell, $\mathrm{V}$ the command potential and $\mathrm{V}_{\text {rev }}$ the reversal potential. This allowed the calculation of the fractional activation of currents as $\mathrm{G} / \mathrm{G}_{\max }$, which could then be fitted by a Boltzmann function of the fol- 
lowing form:

$$
\frac{G}{G_{\text {max }}}=\frac{1}{1+\exp ^{\left(\frac{V_{\text {hal }}-V}{k_{\text {act }}}\right)}}
$$

where $\mathrm{g}_{\max }$ represents the maximal whole cell conductance, $V_{\text {half }}$ the potential of half-maximal activation $\left(\mathrm{G} / \mathrm{G}_{\max }=0.5\right)$ and $k_{a c t}$ the steepness of the activation function, i.e. the slope factor.

\subsubsection{Inactivation}

The extent of Inactivation was obtained by a step depolarization protocol to the voltage of maximal current $\mathrm{V}_{\text {Imax }}$ (approx.-14 $\mathrm{mV}$ in IHCs) for $500 \mathrm{~ms}$. CDI was calculated after normalizing $\mathrm{I}_{C a}$ and $\mathrm{I}_{B a}$ to the maximal current $\left(\mathrm{I}_{\max }\right)$ :

$$
\text { Inactivation }=\frac{R_{500}}{I_{\max }}
$$

where $R_{500}$ represents the residual mean $\mathrm{I}_{C a}$ or $\mathrm{I}_{B a}$ at $490-500 \mathrm{~ms}$ depolarization. CDI is then calculated the following:

$$
C D I=R_{B a, 500}-R_{C a, 500}
$$

\subsubsection{CDI recovery}

To determine recovery from CDI a triple pulse protocol was employed with a $10 \mathrm{~ms}$ pre- and a post-pulse to $-25 \mathrm{mV}$. Before measuring the post pulse a conditioning pulse was applied for 200 $\mathrm{ms}$ at $-14 \mathrm{mV}$ with increasing time intervals at resting $\mathrm{V}_{m}$ between conditioning pulse and post pulse ranging from 4-16-64-256-1024 ms:

$$
C D I_{\text {recovery }}(t)=\frac{I_{P 2}}{I_{P 1}}
$$

$I_{P 1}$ represents the average $\mathrm{I}_{C a}$ from 8-10 ms of the pre-pulse and $I_{P 2}$ the average $\mathrm{I}_{C a}$ from 8-10 ms of the post-pulse. 


\section{Results}

\subsection{CABP2 regulates $\mathrm{Ca}_{V} 1.3$ voltage-gated $\mathrm{Ca}^{2+}$ channels}

$90 \%$ of IHC Ca ${ }^{2+}$ currents are mediated by $\mathrm{Ca}_{V} 1.3 \mathrm{Ca}^{2+}$ channels, which induce stimulus-secretion coupling (Brandt et al., 2003; Dou et al., 2004; Platzer et al., 2000). These $\mathrm{Ca}^{2+}$ channels were shown to be modulated by interacting proteins that interfere with the gating behavior of the channel pore, including activation and inactivation kinetics (Gebhart et al., 2010; Gregory et al., 2013; Neely and Hidalgo, 2014; Yang et al., 2006). Moreover, compared to heterologous expression systems $\mathrm{Ca}_{V} 1.3$ channels in IHCs display weak inactivation kinetics (Yang et al., 2006); however the underlying cause for this discrepancy is not known. To date, two mechanisms were proposed to counteract $\mathrm{Ca}^{2+}$ channel inactivation in IHCs, either the $\mathrm{Ca}^{2+}$ channel itself by the C-terminal modulatory domain (Bock et al., 2011; Cui et al., 2007; Singh et al., 2008) or the interaction with $\mathrm{Ca}^{2+}$ binding proteins (CaBPs, (Yang et al., 2006; Zhou et al., 2005)). The recent identification of a missense mutation in $C A B P 2$ as underlying cause for moderate-to-severe hearing impairment DFNB93 suggests that CaBP2 might predominantly inhibits $\mathrm{Ca}_{V} 1.3$ inactivation in IHCs (Schrauwen et al., 2012). This mutation was predicted to result in translation of a CABP2 protein, completely lacking the C-terminal EF-hand motifs. The expression of such a truncated CABP2 protein in IHCs was suggested to lead to increased $\mathrm{Ca}_{V} 1.3 \mathrm{CDI}$, a hypothesis that required further testing. In addition to this mutation, another nonsense mutation in $C A B P 2$ was identified in hearing impaired subjects, but of unknown pathogenicity. The first set of experiments thus addressed the modulation of $\mathrm{IHC} \mathrm{Ca}_{V} 1.3 \mathrm{Ca}^{2+}$ channels by potentially clinically relevant CABP2 mutant forms compared to wild-type (wt) CABP2. The idea of the experiments was (i) to verify the inhibition of CDI by CABP2-wt, (ii) to check whether newly identified nonsense mutations in CABP2 affect $\mathrm{CABP} 2$ function and (iii) to address specific regulatory functions of $\mathrm{C}$ - or N-terminally located EF-hand domains of CABP2 due to the respective mutations affecting either termini of the protein. A separate role of $\mathrm{N}$ - and $\mathrm{C}$-terminal EF-hand motifs as global and local $\mathrm{Ca}^{2+}$ sensors was previously reported for calmodulin (Faas et al., 2011). I used HEK293 cells stably expressing the human-SK3-1 channel, a $\mathrm{Ca}^{2+}$ inducible $\mathrm{K}^{+}$channel to allow cell repolarization upon $\mathrm{Ca}^{2+}$ entry. Otherwise, HEK293 cells may suffer from excessive $\mathrm{Ca}^{2+}$ entry also called $\mathrm{Ca}^{2+}$ toxicity due to the negative activation potential of $\mathrm{Ca}_{V} 1.3 \mathrm{Ca}^{2+}$ channels (activation threshold at $-45.7 \mathrm{mV}$ (Koschak et al., 2001)), in combination with CABP2, that inhibits channel inactivation. For these recordings, I transiently coexpressed $\mathrm{Ca}_{V} 1.3 \mathrm{Ca}^{2+}$ channel consisting of the pore forming $\mathrm{Ca}_{V} 1.3_{42} \alpha 1$ and the auxiliary subunits $\mathrm{Ca}_{V} 1.3 \beta_{2 A}$ and $\mathrm{Ca}_{V} 1.3 \alpha_{2} \delta$-1 in addition to CABP2-wt or mutant protein (Method 2.3). The $\mathrm{Ca}_{V} 1.3_{42}$ rat clone I used for these experiments was shown to exhibit a point mutation at position 2123 of the distal C-terminus of $\mathrm{Ca}_{V} \alpha 1$, changing the amino acid valin to alanin (Tan et al., 2011). The consequence of this mutation is a more pronounced fast $\mathrm{Ca}^{2+}$-dependent inactivation kinetics of $\mathrm{Ca}_{V} 1.3$, which is comparable to the inactivation kinetics of short $\mathrm{Ca}_{V} 1.3$ isoforms such as $\mathrm{Ca}_{V} 1.3_{42 A}$ or $\mathrm{Ca}_{V} 1.3_{43 S}$ (Bock et al., 2011). I chose to work 
with $\mathrm{Ca}_{V} 1.3_{42}$, as it was used in relevant previous publications inspecting $\mathrm{Ca}_{V} 1.3$ modulations by $\mathrm{CaBPs}$, which enabled me to provide direct comparisons.

I investigated the properties of two different CABP2 mutants. One mutant carries a nonsense mutation affecting the first $\mathrm{Ca}^{2+}$ binding EF-hand in the N-terminal lobe ( CABP2-EF1*, $94 R>G$, Figure $3.1 \mathrm{~A}_{i i}$ ). In parallel, I transfected the already published truncated CABP2 mutant that carries a missense mutation in the inter-lobe linker region of CABP2 $\left(\mathrm{CABP} 2-\mathrm{EF}^{\triangle 3} / 4\right.$, Figure 3.1 $\mathrm{A}_{i i i}$ ). The $\mathrm{Ca}_{V} 1.3_{42}$ channel, with $\mathrm{Ca}_{V} \beta_{2 a}$ and $\mathrm{Ca}_{V} \alpha_{2} \delta-1$ but in the absence of CABP2 was used as control (Methods 2.3). I applied a regular current-voltage (IV) protocol and compared steady-state IV traces (Method 2.15.1). The respective IV-traces are normalized to the cell capac-

A

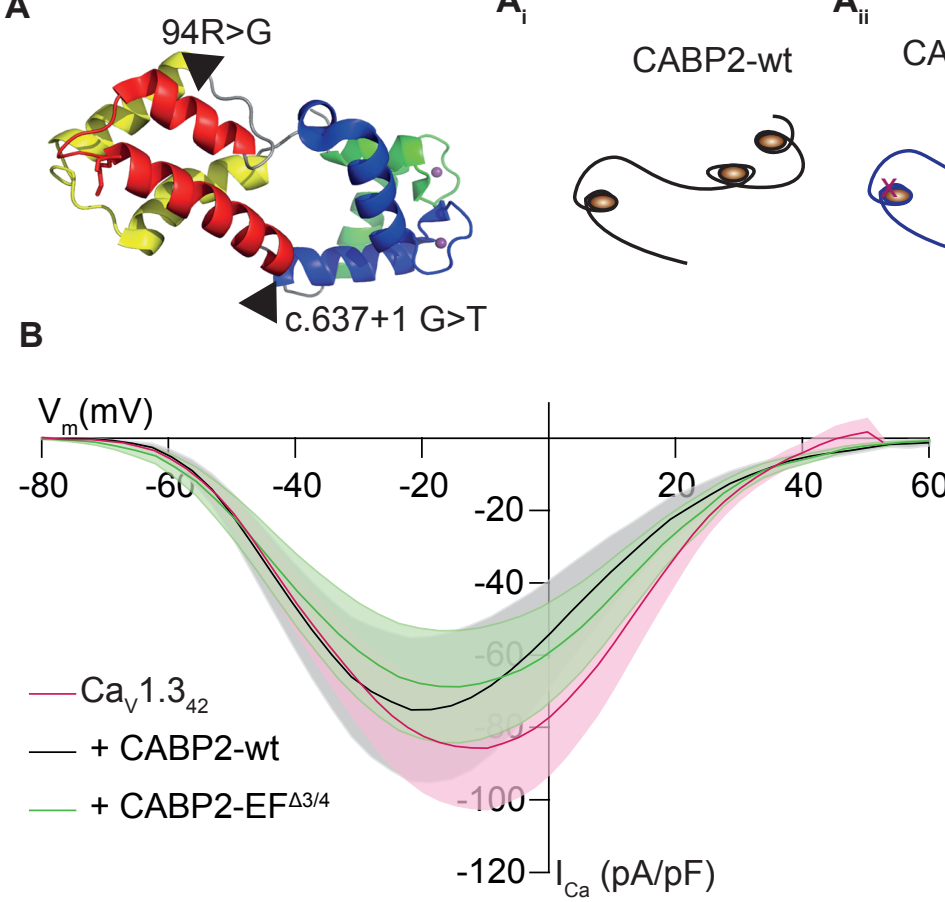

$A_{\text {iii }}$

CABP2-EF1* CABP2-EF ${ }^{\star 3 / 4}$
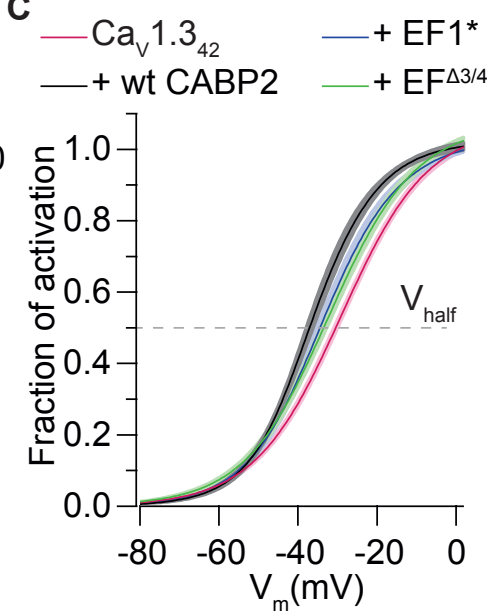

Figure 3.1: CABP2 modulates the biophysical properties of $\mathrm{Ca}_{V} 1.3_{42}:$ (A) Schematic of wt and mutant $\mathrm{CABP} 2$ used throughout the HEK cell experiments. The arrows highlight the mutation sites in human CABP2. The color code is preserved throughout the experiments. (B) Average steadystate IVs normalized to the cell capacitance (current density) of HEK293/hSK3-1 cells transiently cotransfected with either $\mathrm{Ca}_{V} 1.3_{42}$ channels alone $(\mathrm{n}=10)$ or in the presence of CABP2-wt $(n$ $=10)$ and $\mathrm{CABP} 2-\mathrm{EF}^{\Delta 3 / 4}(\mathrm{n}=11)$, respectively. (C) Mean fractional activation curve derived from B including HEK293/hSK3-1 cells coexpressing CABP2-EF1* $(n=11)$. Note the hyperpolarized shift of voltage-dependence in the presence of CABP2-wt and CABP2-EF1* compared to $\mathrm{Ca}_{V} 1.3_{42}$ alone which is less pronounced in recordings in the presence of CABP2-EF ${ }^{\Delta 3 / 4}$.

itance and presented as current densities. The IVs revealed comparable maximal current densities under all four conditions (Figure 3.1B), with shifted voltage-dependence of $\mathrm{Ca}_{V} 1.3_{42}$ activation to 
more hyperpolarized potentials when CABP2-wt is coexpressed. The fractional activation curve (Figure 3.1C) was derived from the IV-curves in B by dividing the linear portion of the IV by a line fit and a subsequent fit to a Boltzmann function.(linear regions for CABP2-wt are 5-10 mV and for $\mathrm{Ca}_{V} 1.3_{42} 5-15 \mathrm{mV}$; see Methods 2.15.1). The traces in $\mathrm{C}$ represent Boltzmann-fit functions in the form of $1 /\left(1+\exp \left(\left(V_{\text {half }}-V\right) / k_{\text {act }}\right)\right)$ for HEK293/hSK3-1 cells cotransfected with either $\mathrm{Ca}^{2+}$ channel alone or in the presence of CABP2-wt, CABP2-EF1* or CABP2-EF ${ }^{43 / 4}$, respectively. The shift in voltage-dependence was induced by an enhanced voltage-sensitivity of the $\mathrm{Ca}^{2+}$ channel in the presence of CABP2-wt as reflected by the reduced activation slope factor $\left(\mathrm{k}_{\text {act }}\right)$. The same applied for CABP2-EF1*. However, CABP2-EF ${ }^{\Delta 3 / 4}$ failed to modify the voltage-sensitivity of $\mathrm{Ca}_{V} 1.3_{42}$ with $\mathrm{V}_{\text {half }}$ and $\mathrm{k}_{a c t}$ values comparable to $\mathrm{Ca}_{V} 1.3_{42}$ alone (Table $3.1)$.

In order to investigate $\mathrm{Ca}_{V} 1.3_{42}$ inactivation, cells were depolarized for $500 \mathrm{~ms}$ to the voltage eliciting the maximum current $\left(\mathrm{V}_{\text {Imax }}\right) \cdot \mathrm{Ca}^{2+}$ or $\mathrm{Ba}^{2+}$ were used as charge carrier, prior to record overall $\mathrm{Ca}^{2+}$ channel inactivation and the latter charge carrier to isolate the voltage-dependent component of inactivation (VDI). The traces were normalized to the peak current $\left(\mathrm{I}_{\max }\right)$ and residual mean currents after $500 \mathrm{~ms}\left(\mathrm{R}_{500}\right)$ were compared (Figure 3.2A,B). CDI was calculated by subtracting $\mathrm{R}_{500, B a}$ from $\mathrm{R}_{500, \mathrm{Ca}} \cdot \mathrm{Ca}_{V} 1.3_{42}$ exhibited very fast CDI kinetics reaching the linear VDI after $20 \mathrm{~ms}$ (Figure 3.2A,B). CABP2-wt however prevented inactivation of $\mathrm{Ca}_{V} 1.3_{42}$, consequently the currents only showed mild VDI. Mutant CABP2-EF1* tended to cause stronger fast inactivation without reaching statistical significance. Truncated CABP2 on the contrary, could only partially prevent CDI.

Next, the same experiments were repeated with CABP2-wt and CABP2-EF ${ }^{\Delta 3 / 4}$ using the corrected long $\mathrm{Ca}_{V} 1.3_{42-A 2123 V}-\alpha 1$ subunit due to the reported expression of this isoform in IHCs ((Scharinger et al., 2015); see constructs in Methods 2.3). As previous data revealed no effect of CABP2-wt or CABP2-EF ${ }^{\Delta 3 / 4}$ on VDI, I focused on $\mathrm{Ca}^{2+}$ currents. Maximal current densities of $\mathrm{Ca}_{V} 1.3_{42-A 2123 V}$ were comparable in the presence or absence of CABP2-wt. However the coexpression of $\mathrm{CABP} 2-\mathrm{EF}^{\Delta 3 / 4}$ induced a reduction in current density amplitude (Figure 3.3A), most probably due to the higher passage/age of the HEK293/h-SK3-1 cells during these recordings. In agreement with data obtained from $\mathrm{Ca}_{V} 1.3_{42}$ experiments, the voltage-sensitivity of $\mathrm{Ca}_{V} 1.3_{42-A 2123 V}$ was increased by CABP2-wt as compared to $\mathrm{Ca}_{V} 1.3_{42-A 2123 V}$ channel alone or with CABP2-EF ${ }^{\Delta 3 / 4}$ (Figure 3.3B). HEK cells expressing $\mathrm{Ca}_{V} 1.3_{42-A 2123 V}$ showed a mean $\mathrm{R}_{500}$ of $0.57 \pm 0.02$ but lacked $\mathrm{Ca}^{2+}$ current inactivation in the presence of CABP2-wt $\left(\mathrm{R}_{500}=0.97 \pm\right.$ 0.005; Figure 3.3C,D). Inactivation of $\mathrm{Ca}_{V} 1.3_{42-A 2123 V}$ with $\mathrm{CABP} 2 \mathrm{EF}^{\Delta 3 / 4}\left(\mathrm{R}_{500}=0.66 \pm 0.03\right)$ was comparable to control thus confirming that CABP2 is dependent on functional C-terminal EF-hands, which likely compete with CaM for CDI modulation. 
A

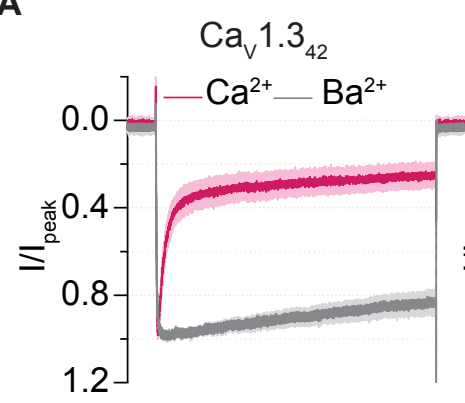

$A_{i}$

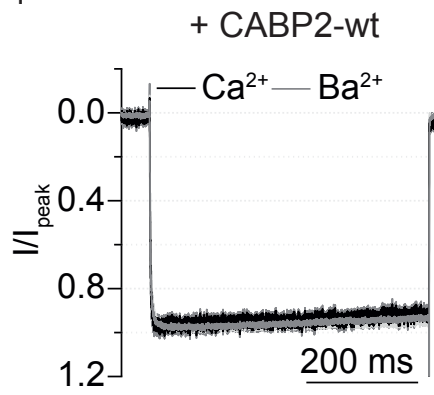

$\mathbf{A}_{\mathrm{ii}}+\mathrm{CABP} 2-\mathrm{EF} 1^{*}$

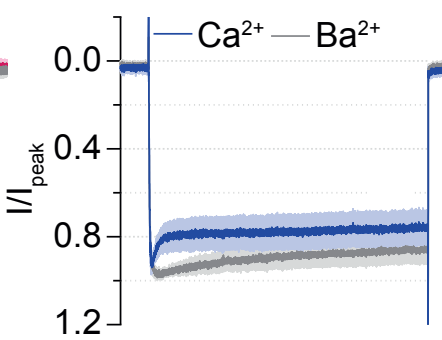

1.2

$A_{\text {iii }}$

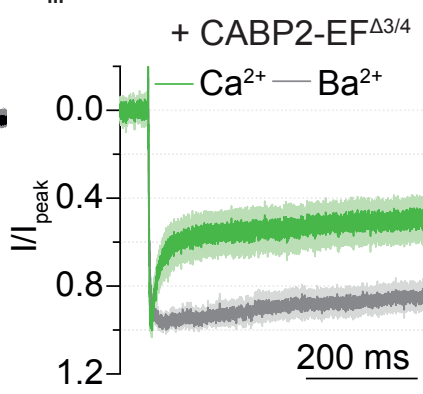

B

- $\mathrm{Ca}^{2+} \square \mathrm{Ba}^{2+}$

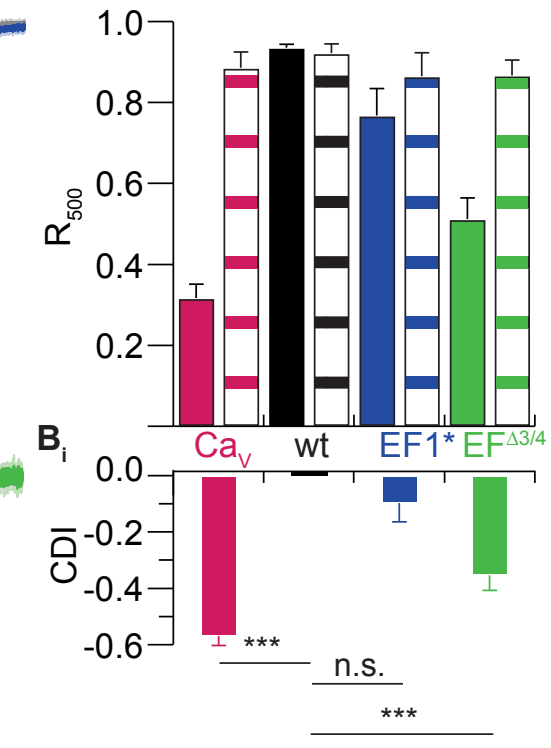

Figure 3.2: CABP2-wt prevents CDI: (for the whole figure: $\mathrm{Ca}_{V} 1.3_{42}$ alone $\left(\mathrm{Ca}^{2+}: \mathrm{n}=10 ; \mathrm{Ba}^{2+}: \mathrm{n}\right.$ $=8)$, CABP2-wt $\left(\mathrm{Ca}^{2+}: \mathrm{n}=10 ; \mathrm{Ba}^{2+}: \mathrm{n}=5\right), \mathrm{CABP} 2-\mathrm{EF} 1 *\left(\mathrm{Ca}^{2+}: \mathrm{n}=13 ; \mathrm{Ba}^{2+}: \mathrm{n}=9\right), \mathrm{CABP} 2-$ $\left.\mathrm{EF}^{\Delta 3 / 4}\left(\mathrm{Ca}^{2+}: \mathrm{n}=11 ; \mathrm{Ba}^{2+}: \mathrm{n}=7\right)\right)(\mathrm{A})$ Normalized average traces of $\mathrm{Ca}_{V} 1.3_{42} \mathrm{Ca}^{2+}$ and $\mathrm{Ba}^{2+}$ currents during $500 \mathrm{~ms}$ depolarizations. Colored traces represent $\mathrm{I}_{C a}$, gray traces $\mathrm{I}_{B a}$. for $\mathrm{Ca}_{V} 1.3_{42}$ alone (A), CABP2-wt $\left(\mathrm{A}_{i}\right), \mathrm{CABP} 2-\mathrm{EF} 1 *\left(\mathrm{~A}_{i i}\right)$ and CABP2-EF ${ }^{\Delta 3 / 4}\left(\mathrm{~A}_{i i i}\right)$. (B) $\mathrm{R}_{500, B a}$ and $\mathrm{R}_{500, C a}$ derived from $\mathrm{A}$. $\mathrm{R}_{500}$ represents the residual current after $500 \mathrm{~ms}$ depolarization. $\mathrm{R}_{500, \mathrm{Ca}}$ are demonstrated by filled and $\mathrm{R}_{500, B a}$ by striped bars. $\left(\mathrm{B}_{i}\right)$ CDIs are colored in the respective color code according to the constructs used. CABP2-wt and CABP2-EF1* inhibit CDI, which is less pronounced in the presence of CABP2-EF ${ }^{\Delta 3 / 4}$.

Table 3.1: Biophysical properties of $\mathrm{Ca}_{V} 1.3$ in the presence or absence of CABP2-wt or CABP2mutant expressed in HEK293/hSK3-1 cells

\begin{tabular}{lllllll}
\hline & $\mathbf{V}_{\text {half }}(\mathbf{m V})$ & $p_{C a V}$ & $p_{w t}$ & $\mathbf{k}_{a c t}(\mathbf{m V})$ & $p_{C a V}$ & $p_{w t}$ \\
\hline \hline $\mathrm{Ca}_{V} 1.3_{42}$ & $-29.1 \pm 0.8$ & & & $10.9 \pm 0.3$ & & \\
+ CABP2-wt & $-36.3 \pm 1.1$ & $<0.005$ & & $6.7 \pm 0.3$ & $<0.005$ & \\
+ CABP2-EF1 & $-33.4 \pm 1.4$ & 0.013 & 0.054 & $9.5 \pm 0.5$ & 0.006 & 0.718 \\
+ CABP2-EF & $-31.9 \pm 1.1$ & 0.137 & 0.016 & $10.7 \pm 0.5$ & 0.122 & 0.078 \\
& & & & & & \\
$\mathrm{Ca}_{V} 1.3_{42-A 2123 V}$ & $-20.8 \pm 1.1$ & & & $9.7 \pm 0.2$ & & \\
+ CABP2-wt & $-35.4 \pm 1.5$ & $<0.005$ & & $6.7 \pm 0.3$ & $<0.005$ & \\
+ CABP2-EF & $-18.8 \pm 1.5$ & 0.98 & $<0.005$ & $9.7 \pm 0.2$ & 0.42 & $<0.005$ \\
\hline
\end{tabular}


A

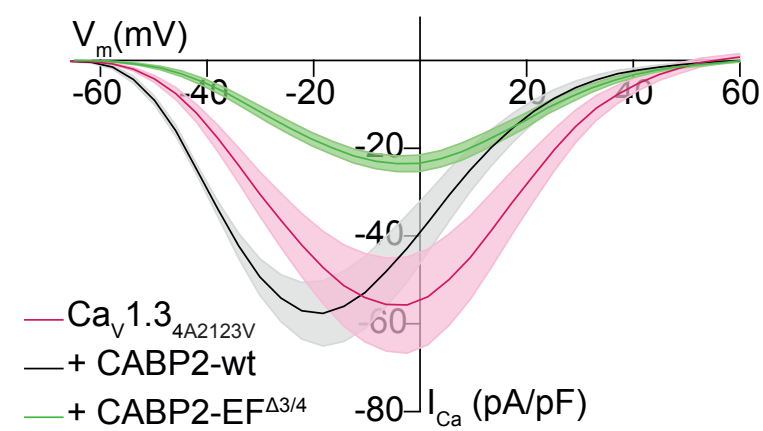

C

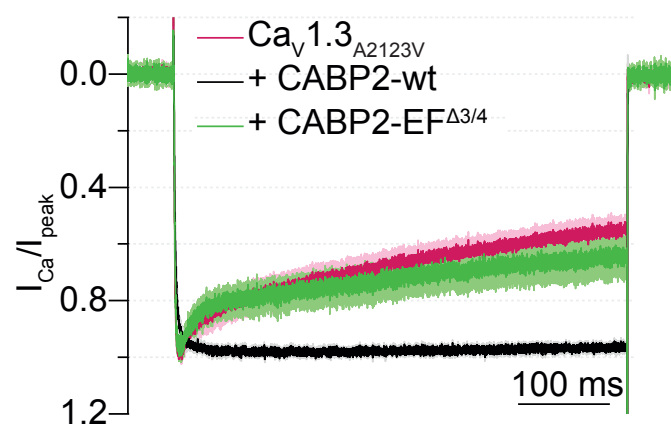

B
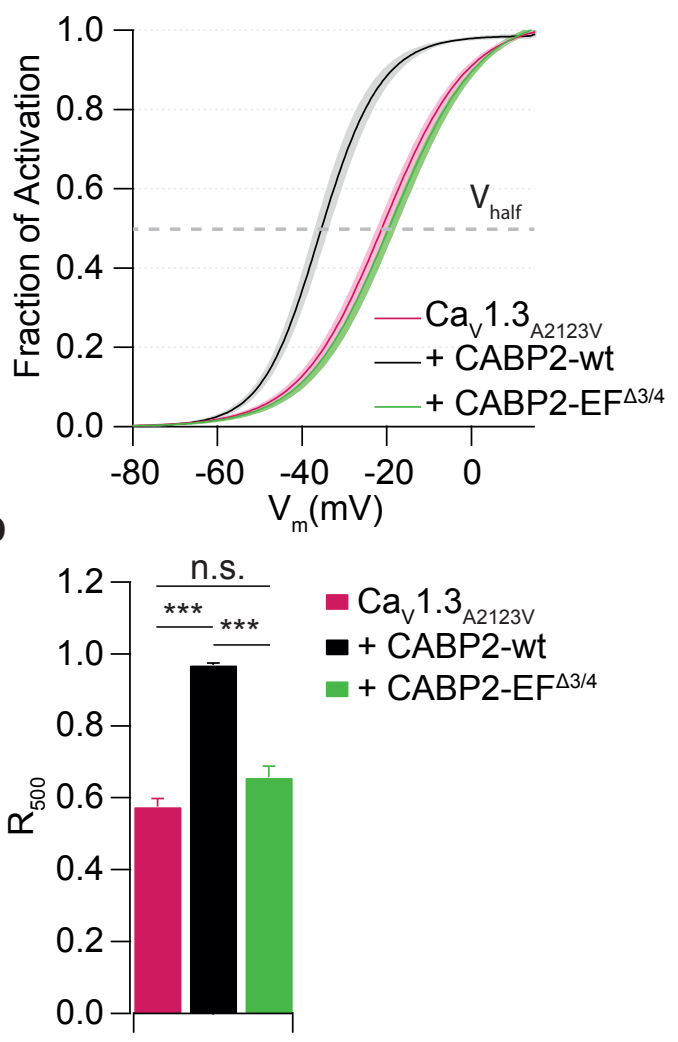

Figure 3.3: $\mathrm{CABP} 2$ modulates $\mathrm{Ca}_{V} 1.3_{42-A 2123 V}$ gating: (for the whole figure: $\mathrm{Ca}_{V} 1.3_{42-A 2123 V}$ $\left.(\mathrm{n}=9), \mathrm{CABP} 2-\mathrm{wt}(\mathrm{n}=9), \mathrm{CABP} 2-\mathrm{EF}^{\Delta 3 / 4}(\mathrm{n}=11)\right)(\mathrm{A})$ Comparable peak currents recorded in HEK/hSK3-1 cells expressing $\mathrm{Ca}_{V} 1.3_{42-A 2123 V}$ and in the presence of CABP2-wt. Current density amplitudes are reduced when CABP2-EF ${ }^{\Delta 3 / 4}$ is coexpressed. (B) Average activation curves derived from A. Note that the activation curve is significantly shifted to more hyperpolarized potentials by CABP2-wt but not $\mathrm{CABP} 2-\mathrm{EF}^{\Delta 3 / 4}$. (C) Normalized $\mathrm{I}_{C a}$ during $500 \mathrm{~ms}$ depolarization for CABP2-wt, $-\mathrm{EF}^{\Delta 3 / 4}$ and $\mathrm{Ca}_{V} 1.3_{42-A 2123 V}$ control recordings. (D) $\mathrm{R}_{500, C a}$ derived from $\mathrm{C}$ demonstrating inactivation of $\mathrm{Ca}_{V} 1.3_{42-A 2123 V}$. CABP2-wt is abolishing $\mathrm{Ca}^{2+}$ channel inactivation, while $\mathrm{CABP} 2-\mathrm{EF}^{\Delta 3 / 4}$ is not able to stabilize the opening of the channels revealing comparable $\mathrm{R}_{500, C a}$ to $\mathrm{Ca}_{V} 1.3_{42-A 2123 V}$ control.

Table 3.2: Inactivation of $\mathrm{Ca}_{V} 1.3$ in the presence or absence of CABP2-wt or CABP2-mutant expressed in HEK293/hSK3-1 cells

\begin{tabular}{lllll}
\hline & VDI & CDI & $\begin{array}{l}p_{C a V} \\
\text { VDI;CDI }\end{array}$ & $\begin{array}{l}p_{w t} \\
\text { VDI;CDI }\end{array}$ \\
\hline \hline $\mathrm{Ca}_{V} 1.3_{42}$ & $-0.11 \pm 0.04$ & $-0.57 \pm 0.03$ & & \\
$+\mathrm{CABP} 2-w t$ & $-0.08 \pm 0.02$ & $-0.01 \pm 0.01$ & $0.14 ;<0.005$ & - \\
$+\mathrm{CABP} 2-\mathrm{EF} 1^{*}$ & $-0.13 \pm 0.06$ & $-0.10 \pm 0.07$ & $0.57 ;<0.005$ & $0.74 ; 0.90$ \\
$+\mathrm{CABP}^{-E^{\Delta 3} / 4}$ & $-0.13 \pm 0.04$ & $-0.35 \pm 0.05$ & $0.21 ; 0.006$ & $0.24 ;<0.005$ \\
\hline
\end{tabular}




\subsection{Cabp2 $2^{L a c Z / L a c Z}$ as a model for DFNB93}

To elucidate the function of Cabp2 in sound encoding at the IHC synapse and unravel the disease mechanisms underlying DFNB93 a Cabp2 mutant mouse model was generated. Mouse ES cells carrying a null allele of Cabp2 were provided by KOMP repository (CSD50029,DEPD0003_7_F06). Dr. Aleksandra Ivanovic (MPI-EM, Göttingen) confirmed the integrity of the knock-out cassette by long range PCR (LR-PCR) of the 5' and the 3' target alleles (Figure 3.4). The sequenced areas included 5' and 3' insertion sites, FRT recombination site and loxP sites (Figure 3.4A).

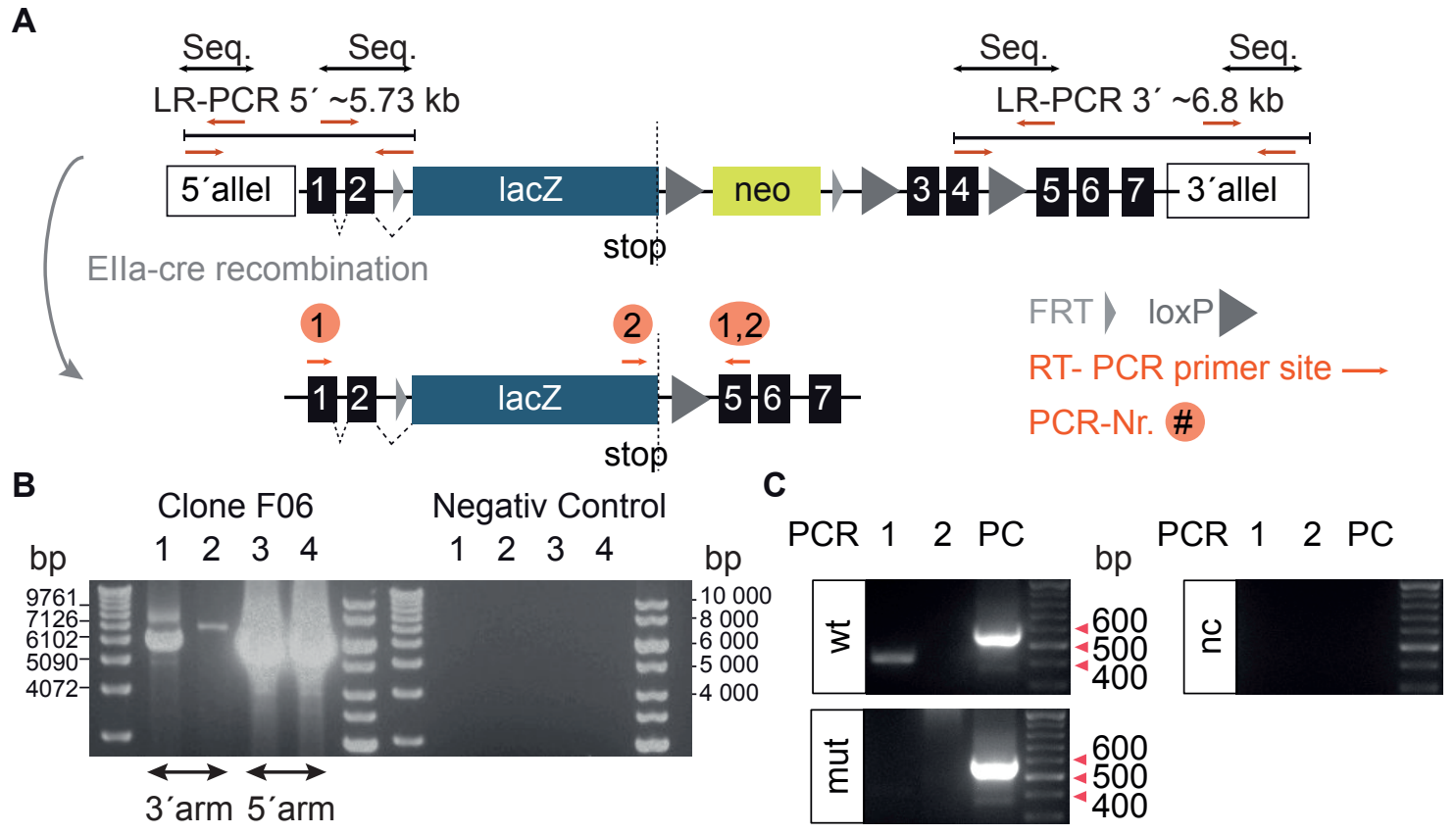

Figure 3.4: Confirmation of sequence integrity and Cabp2 mutation by an IRES:LacZ trapping cassette: (A) Schematic drawing of sequencing and reverse transcription PCR (RT-PCR) strategies. LR-PCR primer sites are illustrated by black arrows, the amplicon by the black line and the sequenced fragments by the black double arrows flanked by the sequencing primers in orange (upper). Schematic of IRES:LacZ trapping cassette after Cre-recombination (lower). Orange arrows highlight the binding sites of the RT-PCR primers, confirmed fragments are indicated with a PCR number.(B) DNA bands of the LR-PCR fragments of LR-PCR 1,2 for the 3' target allele and 3,4 for the 5' target allele. Note that the DNA fragments of LR-PCR 1-4 are present in all lanes. (C) Agarose gel of amplified cDNA of Cabp2 $2^{+/+}$(wt) and Cabp2 $2^{\mathrm{LaCZ} / \mathrm{LaCZ}}$ (mut) organs of Corti. RT-PCR DNA fragments to confirm Cabp 2 mutation: $C a b p 2^{+/+}$cDNA contains the expected wild-type band amplified by RT-PCR 1 (long Cabp2: 418 bp), which is absent in Cabp2 $2^{\text {LacZ/LacZ }}$ cDNA. RT-PCR 2 for the detection of spontaneously recombinated rest Cabp2 expression is negative in $\mathrm{Cabp2}^{+/+}$and $\mathrm{Cabp} 2^{\mathrm{LacZ} / \mathrm{LacZ}}$, while the amplification of GAPDH cDNA as positive control (pc) is detected in both samples (572 bp).

The expected PCR fragments for LR-PCR 1-4 were identified (amplicon sizes: $6.053 \mathrm{~kb}, 6.846$ kb, $5.733 \mathrm{~kb}, 5.731 \mathrm{~kb}$; Figure 3.4B) and confirmed by sequencing. Cultured ES cells were then 
injected into the blastocyst of C57BL/6N mice, resulting in chimeric animals. Heterozygous animals of the F1 generation were back-crossed to ensure germ-line transduction of the IRES:Lacz Cabp 2 trapping cassette. Heterozygous mice of F3 generation were crossbred with EIIAcre mice that express Cre-recombinase under the promoter of the transcription factor EIIa (Lakso et al., 1996). Cre positive Cabp2 $2^{\text {LacZ/LacZ }}$ animals showed global and constitutive recombination and excision of exon 3 and 4 and the neomycin resistance sequence, stabilizing the Cabp 2 trapping mutation (Skarnes et al., 2011). In Cabp2 $2^{\text {LacZ } / L a c Z}$ mice exon 1 and 2 of Cabp 2 were expressed followed by a $\beta$-galactosidase tag, which was working as null mutation to compromise Cabp2 functionality and acted as reporter for Cabp2 gene expression. The mouse colony showed no obvious signs of behavioral, size and weight abnormalities or decreased lifespan in either gender. Breeding was normal and genotypes of the offspring followed Mendelian inheritance.

To verify the absence of Cabp2 in the mutants, western blotting and immunostainings of Cabp2 $2^{\text {LacZ/LacZ }}$ organs of Corti were performed. Since current commercially available antibodies showed either no or unspecific detection signals in both types of experiments, I aimed to generate a Cabp2-specific antibody in collaboration with the company Synaptic Systems. The high sequence homology between $\mathrm{CaBP} 2$ and $\mathrm{CaM}$ or other CaBPs restricted the selection of a suitable recognition site resulting in an antibody with a Cabp2 unspecific epitope. As alternative Cabp2 detection strategy I used RT-PCR to screen mRNA of apical turns of six organs of Corti isolated from three Cabp2 $2^{\mathrm{LaCZ} / \mathrm{LacZ}}$ and Cabp2 $2^{+/+}$animals. To verify Cabp2 expression in Cabp2 $2^{+/+}$animals, primer pairs spanning exon 1 to exon 5 were designed (Figure 3.4A, lower; RT-PCR 1). With this approach one can not only test Cabp2 expression, but also distinguish between the expressed isoforms. Due to the lack of exon 2 in the short Cabp2 isoform the difference of amplicon length amounts to $159 \mathrm{bp}$, which is easily detectable (259 vs. $418 \mathrm{bp}$ ). In Cabp2 $2^{+/+}$mice mRNA of the long Cabp2 isoform was detected, which was absent in mRNA isolated from Cabp2 $2^{\text {LacZ/LacZ }}$ cochleae (Figure 3.4C). To confirm the transcription termination after the IRES:LacZ sequence, a primer pair spanning the C-terminal end of LacZ and exon 5 (Figure 3.4A,C; PCR 2) confirmed the proper function of the IRES:LacZ trapping system. The expression of the housekeeping gene glyceraldehyde-3-phosphate dehydrogenase (GAPDH) cDNA in Cabp2 $2^{+/+}$and Cabp2 $2^{\mathrm{LaCZ} / \mathrm{LacZ}}$ confirmed successful isolation of the organ of Corti cDNA (Figure 3.4C; PCR PC).

\subsubsection{Indirect approach to detect Cabp2 expression in $\operatorname{Cabp2} 2^{L a c Z / L a c Z}$ mice}

Due to the lack of specific antibodies against Cabp2 I took advantage of the LacZ reporter and performed $\beta$-galactosidase stainings of the cochlea and the retina, to study Cabp 2 expression. The respective tissues were selected based on previous work suggesting that Cabps are predominantly expressed in sensory cells and neurons (Cui et al., 2007; Kim et al., 2014; Sokal et al., 2000).

These stainings revealed Cabp2 expression in inner and outer hair cells of the organs of Corti 

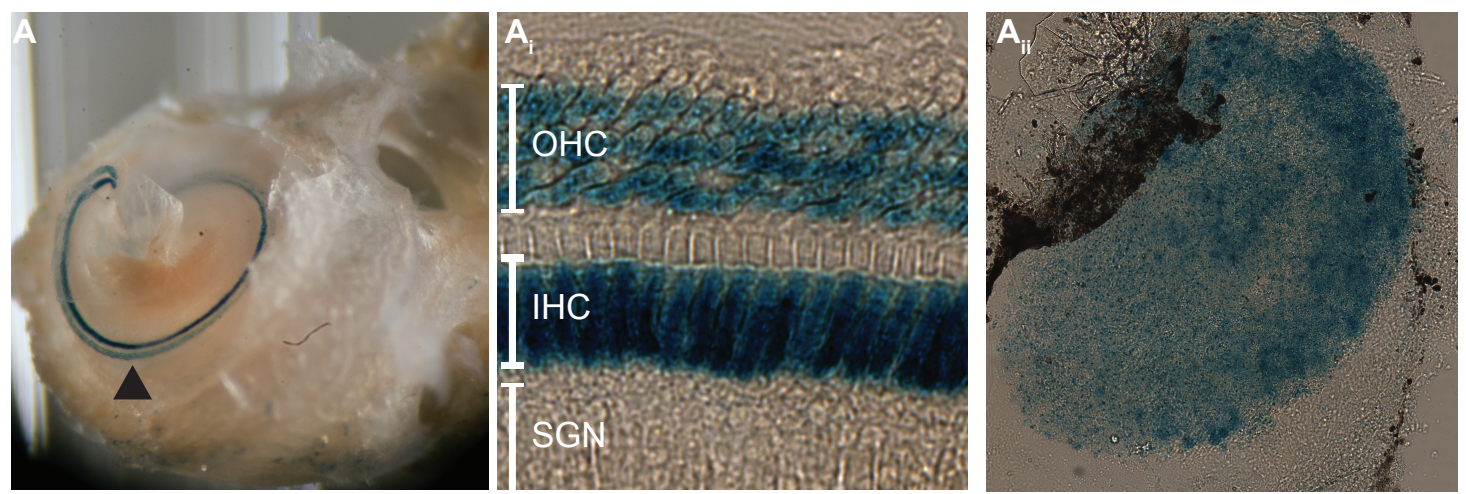

B
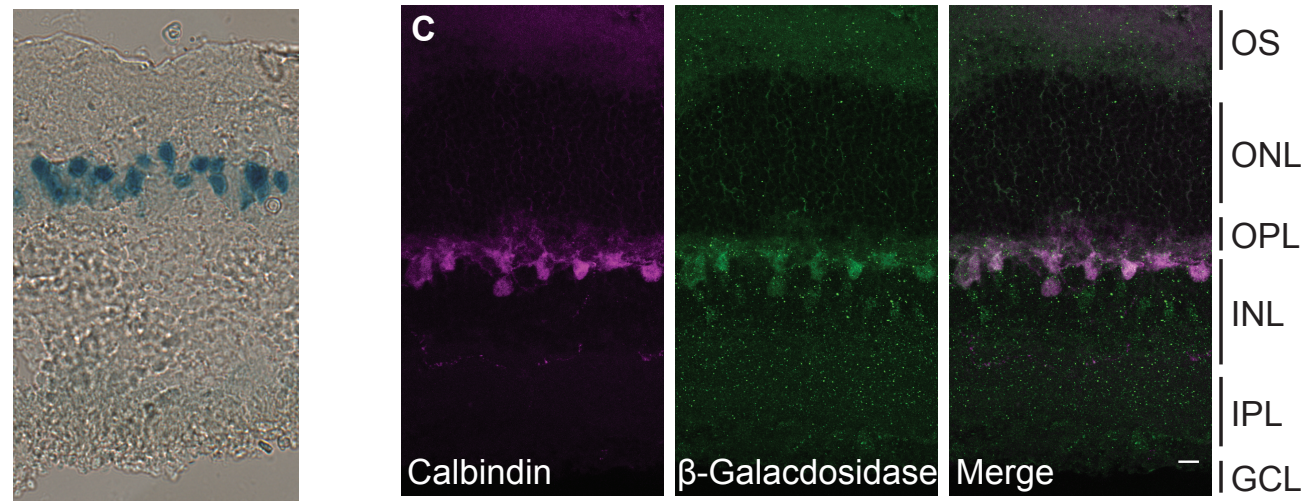

Figure 3.5: Cabp2 is expressed in cochlear hair cells and in retinal cells: (A) Image of a mouse cochlea after LacZ staining. The black arrow highlight HCs of the organ of Corti. $\left(\mathrm{A}_{i}\right)$ Magnification of the organ of Corti showing Cabp2 expression in IHCs and OHCs. $\left(\mathrm{A}_{i i}\right)$ Whole mount of utricle with evenly distributed LacZ positive vestibular HCs.(B) $\beta$-galactosidase stained section of $C a b p 2^{\text {LacZ/LacZ }}$ retinal slice. (C) Immunohistochemical staining of retinal slice showing horizontal and amacrine cells stained by calbindin (magenta) and indirect Cabp2 expression by anti- $\beta$-galactosidase (green) antibodies. The Gross laminar distribution is indicated: outer segment (OS), outer nuclear layer (ONL), outer plexiform layer (OPL), inner nuclear layer (INL), inner plexiform layer (IPL) and ganglion cell layer (GCL). Scale bar corresponds to $10 \mu \mathrm{m}$.

(Figure $3.5 \mathrm{~A}_{i}$ ) with a stronger blue signal in IHCs. In addition, peripheral vestibular sensory organs displayed evenly distributed LacZ positive HCs, as illustrated by a whole mount of the utricle (Figure 3.5A $\mathrm{A}_{i i}$ ). Moreover, in the retina, cells most probably of the inner retinal layers were positive for the indigo dye (Figure 3.5B). To further identify Cabp2 expressing cell types in the retina immunohistochemistry was performed. To localize Cabp2 expression I used a $\beta$-galactosidase antibody and calbindin, a marker for amacrine and horizontal cells. The staining in both channels appeared to overlap, indicating that Cabp2 is expressed in these cell types (Figure 3.5C). 


\subsubsection{Moderate hearing impairment in $\operatorname{Cabp} 2^{L a c Z / L a c Z}$ mice}

Hearing performance of $\mathrm{Cabp} 2^{\mathrm{LacZ} / \mathrm{LacZ}}$ mice was assessed by probing cochlear amplification and neuronal activity along the auditory pathway. For the $\mathrm{OHC}$ function distortion product otoacoustic emissions (DPOAEs) were measured, whereas auditory brainstem recordings (ABRs) were used to recording synaptic transmission. Hearing was tested in animals of three age groups: 3-, 5-, and 7-8-week-old animals, respectively (experiments performed by the technician Stefan Thom). DPOAEs were present in $\mathrm{Cabp} 2^{\mathrm{LacZ} / \mathrm{LacZ}}$ mice at all tested ages and were comparable to littermate control animals. Figure 3.6D shows exemplary mean \pm s.e.m. DPOAE intensities as a function of the intensity of the second tone $\left(\mathrm{f}_{2}\right)$ for $C a b p 2^{\text {LacZ/LacZ }}$ and Cabp2 $2^{+/+}$animals. These recordings confirm intact cochlear amplification mediated by $\mathrm{OHC}$ elongation in the absence of full length Cabp2 indicating that the hearing impairment DFNB93 does arise downstream of OHCs. Next, ABR-click responses were obtained at 20 and $80 \mathrm{~Hz}$ to assess hearing thresholds in a stimulationrate dependent manner. Figure 3.6A illustrates ABR waveforms with the four-five characteristic ABR peaks for 3-, 5- and 8-week old animals. Already in young (3-week-old) Cabp2 deficient mice a significant decrease in wave I amplitude at $20 \mathrm{~Hz}$ was observed $(\mathrm{p}<0.01$, Student's t-test). At the age of 8 weeks I observed a further reduction in wave I amplitudes $(\mathrm{p}<0.001$, Student's t-test; Figure $3.6 \mathrm{~A}_{i i}$ ) and a delay of ABR wave I ( $<<0.01$ at $40 \mathrm{~dB}$, Student's t-test; $\mathrm{p}<0.001$ at 50-100 dB, Student's t-test). The same trend applied to ABR thresholds, where already 3-weekold animals showed significantly elevated hearing thresholds in the frequency range of $8-16 \mathrm{kHz}$, which increased and expanded over a wider frequency range $(8-24 \mathrm{kHz})$ in 5 - and 8-week-old animals (Figure 3.6B). On the other hand, ABR thresholds of heterozygous Cabp2 $2^{+/ L a c Z}$ were comparable to those of control mice (Figure 3.6C).

Since Cabp2 was expressed in the retina, Stefan Thom also recorded scotopic electoretinogramms (ERGs) to test vision of dark adapted anesthetized mice. Preservation of A and B-wave amplitudes illustrate that Cabp2 disruption has no significant effect on the rod system, implying that the signal transmission between photoreceptor, bipolar and ganglion cells is most likely intact (Figure 3.7).

In summary, Cabp2 deficient mice experience recessive moderate hearing loss that progresses with age as illustrated by a decrease in the ABR amplitudes of wave I and an increase in ABR thresholds. The hearing impairment did not involve a deficit in cochlear amplification since DPOAEs were normal.

\subsubsection{IHC development is not affected in the absence of functional Cabp2}

The hearing impairment increase might reflect a developmental deficit and the progressive loss of ribbon synapses. This is not unlikely, since impaired synaptogenesis of photoreceptor termini was reported in Cabp4 knock-out mice, resulting in a narrower outer plexiform layer (Haeseleer et al., 2004; Maeda et al., 2005). Therefore, I checked basic developmental and morphological charac- 
A

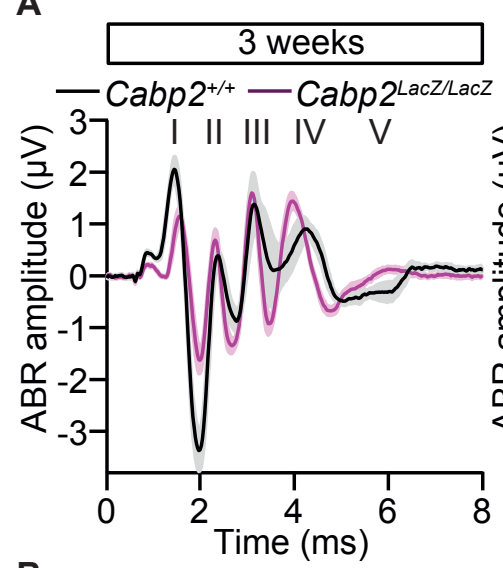

B
$A_{i}$

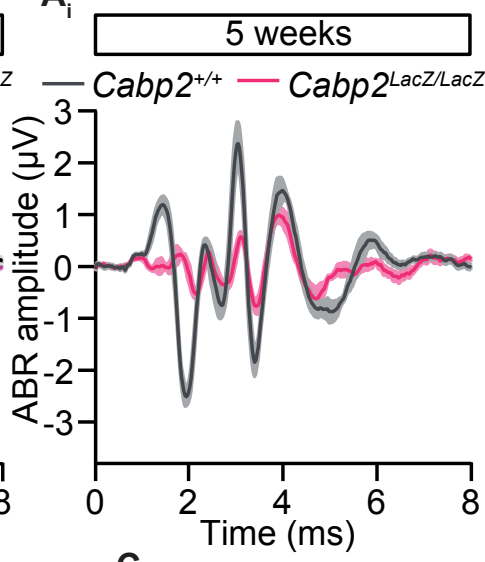

C

$\rightarrow$ Cabp2 $2^{+/+}, 8 \mathrm{w} \rightarrow-\mathrm{Cabp}^{\mathrm{LacZ} / L a c Z}, 8 \mathrm{w}$

- Cabp2 $2^{++}, 5 \mathrm{w} \rightarrow-$ Cabp2LaczLacz, $5 \mathrm{w}$

$\rightarrow-$ Cabp2 $^{++}, 3 \mathrm{w} \rightarrow \mathrm{Cabp2}^{\text {LacZ/Lacz, }}, 3 \mathrm{w}$
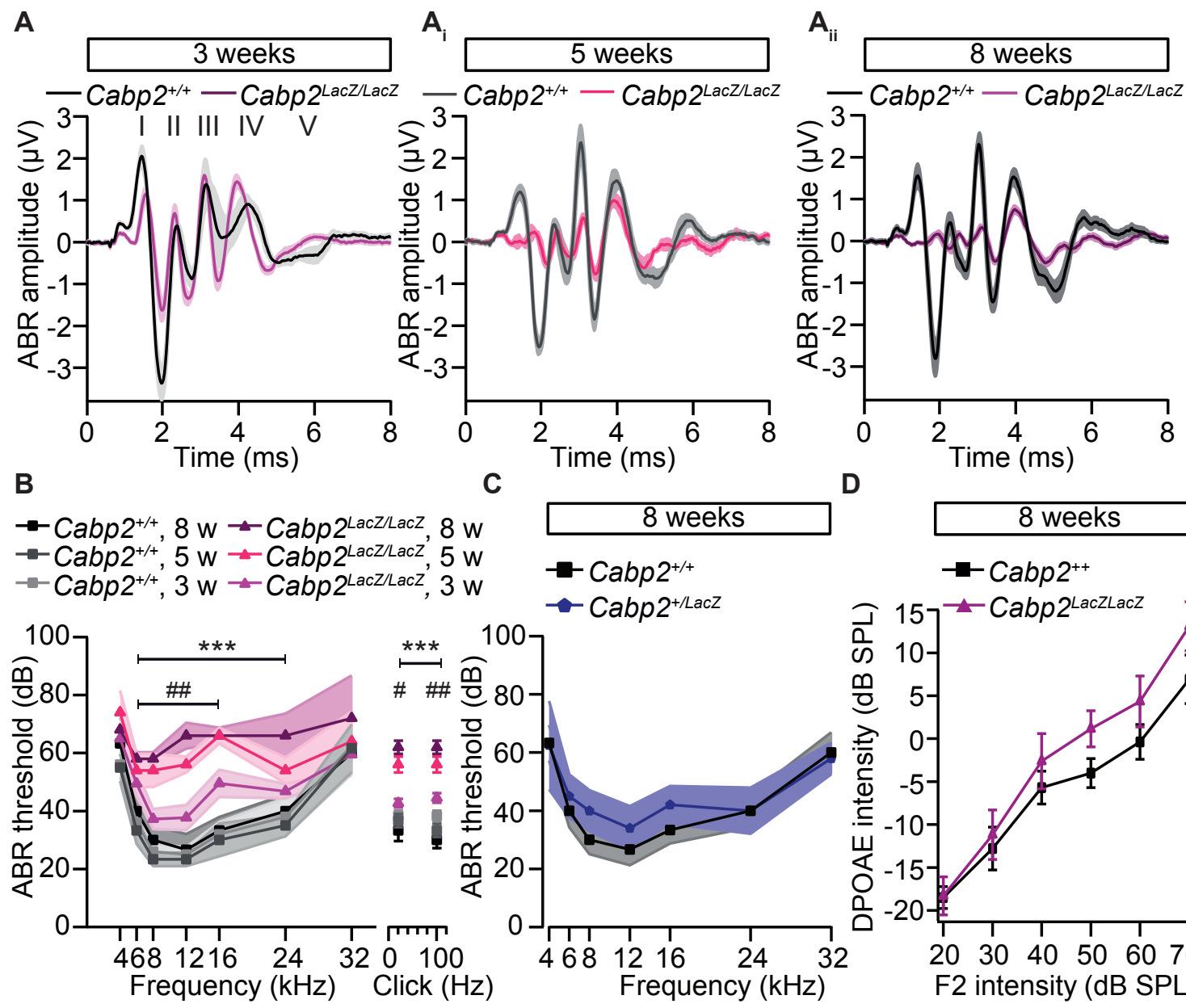

D

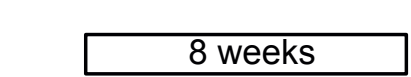

Cabp2 $2^{+++}$

$\rightarrow$ Cabp2 $2^{+/ L a c Z}$
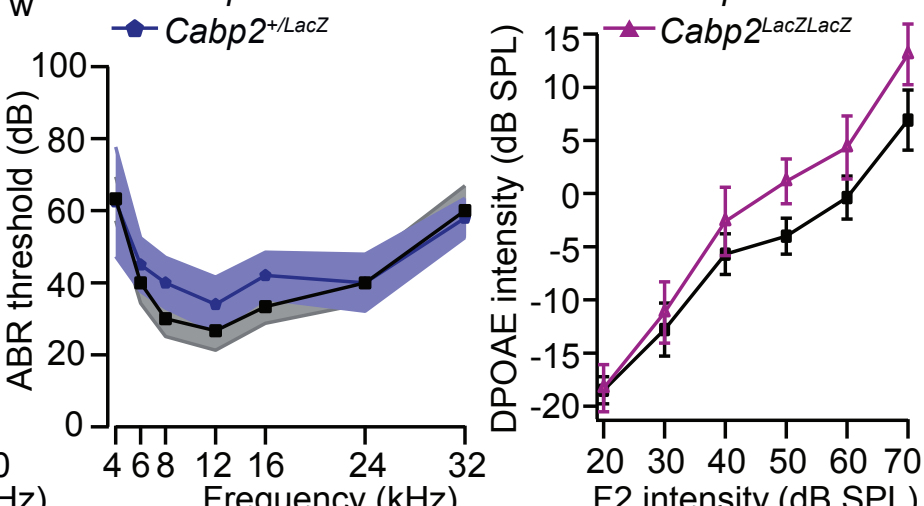

Figure 3.6: $C a b p 2^{L a c Z / L a c Z}$ mice exhibit progressive hearing loss: (A) Grand average of ABR waves elicited by $80 \mathrm{~dB}$ click stimuli at a sampling rate of $20 \mathrm{~Hz}$ in $C a b p 2^{\mathrm{LacZ} / \mathrm{LaCZ}}$ and Cabp2 $2^{+/+}$ animals of $3\left(C_{\text {Cabp }} 2^{+/+}: \mathrm{n}=10, C_{\text {Cabp }}{ }^{\text {LacZ/LacZ }}: \mathrm{n}=11, \mathrm{~A}\right), 5\left(\mathrm{Cabp2}^{+/+}: \mathrm{n}=8\right.$, Cabp2 $\left.{ }^{\text {LacZ/LacZ }}: \mathrm{n}=9 \mathrm{~A}_{i}\right)$, and $8\left(C_{\text {Cabp }} 2^{+/+}: \mathrm{n}=9, C_{a b p 2^{\text {LacZ } / L a c Z}} \mathrm{n}=8 \mathrm{~A}_{i i}\right)$ weeks of age. Roman numerals represent ABR peaks of neuronal discharges along the auditory pathway. Note the age-dependent reduction and delay of ABR wave I amplitude in Cabp2 $2^{\mathrm{LaCZ} / \mathrm{LacZ}}$ animals compared to their respective littermate controls. (B) ABR thresholds of Cabp2 $2^{+/+}$and $C a b p 2^{\mathrm{LacZ} / \mathrm{LacZ}}$ animals in response to tone bursts recorded in a frequency range of 4-32 $\mathrm{kHz}$. In Cabp2 $2^{\mathrm{LacZ} / \mathrm{LacZ}}$ animals ABR threshold elevation progresses with age. For clarification only p-values of 3 (\#)- and $8(*)$ - week-old animals are indicated. (C) Comparable ABR thresholds of 8 -week-old Cabp2 $2^{+/+}$ (same recordings as in $\mathrm{A}$ ) and $\mathrm{Cabp} 2^{+/ \mathrm{LacZ}}(\mathrm{n}=5)$ animals. (D) Representative average distortion product of $\mathrm{f} 1=9.4 \mathrm{kHz}$ and $\mathrm{f} 2=11.3 \mathrm{kHz}$ stimuli for Cabp $2^{\text {LacZ/LacZ }}$ and Cabp2 $2^{+/+}$animals. DPOAEs are not altered in Cabp2 $2^{\text {LacZ/LacZ }}$ mice compared to Cabp2 $2^{+/+}$control.

teristics of IHCs, such as cell number and size, as well as synapse number. Throughout this study, I never observed obvious IHC loss in Cabp $2^{\text {LacZ/LacZ }}$ organs of Corti as demonstrated in Figure 3.8A. IHC expression and morphology appeared comparable between the genotypes. Further, the IHC size of p35-42 animals estimated by $\mathrm{C}_{\text {slow }}$ during whole-cell patch clamp recordings was com- 

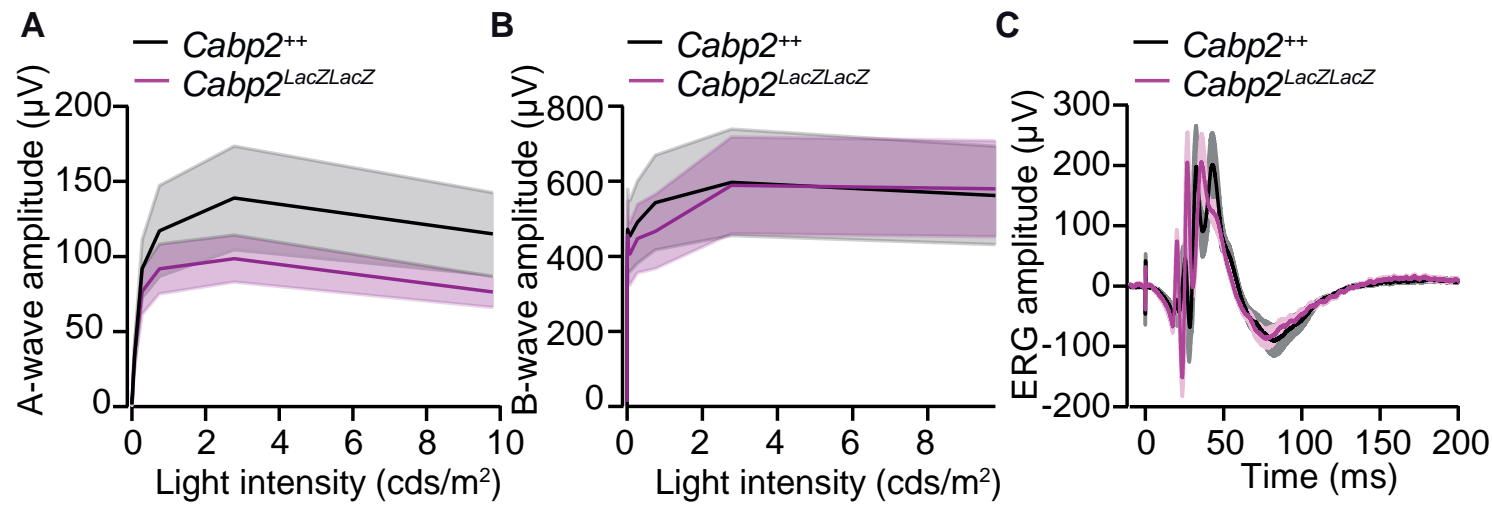

Figure 3.7: Scotopic ERGs in Cabp2 $2^{\mathrm{LacZ} / \mathrm{LacZ}}$ animals are normal: (A) Grand average of Awave amplitudes is comparable between $\operatorname{Cabp} 2^{\text {LacZ/LacZ }}(\mathrm{n}=4)$ and $C a b p 2^{+/+}$animals $(\mathrm{n}=$ 4). (B) B-wave amplitudes are not affected in Cabp2-deficient mice. (C) Electroretinogramm of $\mathrm{Cabp} 2^{\mathrm{LacZ} / \mathrm{LacZ}}$ and $\mathrm{Cabp} 2^{+/+}$animals from A,B with comparable negative deflection of mean A-wave and subsequent positive deflection of B-wave.

parable in $C a b p 2^{\text {LacZ/LacZ }}$ and Cabp2 $2^{+/+}$mice $\left(C_{a b p} 2^{+/+}: 9.2 \pm 0.0 \mathrm{pF}, \mathrm{n}=23 ; C_{a b p 2^{L a c Z / L a c Z}}\right.$ $: 8.7 \pm 0.0 \mathrm{pF}, \mathrm{n}=27 ; \mathrm{p}=0.13$, Student's t-test). To investigate morphological features of ribbon synapse integrity and stability in more detail, I performed immunofluorescence of IHCs and their ribbon synapses as described previously (Khimich et al., 2005; Meyer et al., 2009). The organs of Corti were stained for the presynaptic ribbon with an anti-Ctbp2 antibody (magenta) and the postsynaptic glutamate receptors 2 and 3 (AMPA receptors GluA2/3, green). Juxtaposed Ctbp2 and GluA2/3 puncta were counted as ribbon synapses (Schematic and representative stainings in Figure 3.8B). The number of ribbon synapses per cell of $\mathrm{Cabp}^{+/+}$and $\mathrm{Cabp} 2^{\mathrm{LaCZ} / \mathrm{LacZ}} \mathrm{IHC}$ was comparable for animals of both age groups ( 3 weeks, 8 weeks; Figure 3.8C; p > 0.05, Student's t-test). Moreover, $\mathrm{I} \mathrm{Ca}_{V} 1.3$ channel clustering at the ribbon synapses was tested, showing normal $\mathrm{Ca}_{V} 1.3$ channel cluster formation in 8-week-old animals (Figure 3.8D).

This very basic experiment indicated normal development and normal maintenance of IHC ribbon synapses in Cabp2 $2^{\mathrm{LaCZ} / \mathrm{LacZ}}$ mice.

\subsubsection{Voltage-sensitivity of IHC Ca ${ }^{2+}$ influx is not altered in $\mathrm{Cabp}^{\mathrm{LacZ} / L a c Z}$ mice}

Heterologous expression experiments in HEK293/hSK3-1 cells depicted two not mutually exclusive candidate mechanisms for DFNB93: (i) a reduction in sensitivity of IHC $\mathrm{Ca}_{V} 1.3$ channels towards graded changes of the physiological receptor potential and (ii) an increase of steady-state inactivation of IHC $\mathrm{Ca}_{V} 1.3$ channels. To probe the voltage-sensitivity of Cabp2 ${ }^{\mathrm{LacZ} / \mathrm{LacZ}} \mathrm{IHCs}$, a standard IV protocol with $20 \mathrm{~ms}$ depolarizations and a $\Delta \mathrm{V}$ of $5 \mathrm{mV}$ was used and the steadystate IV curves were derived. All recordings were performed in perforated-patch configuration 
A

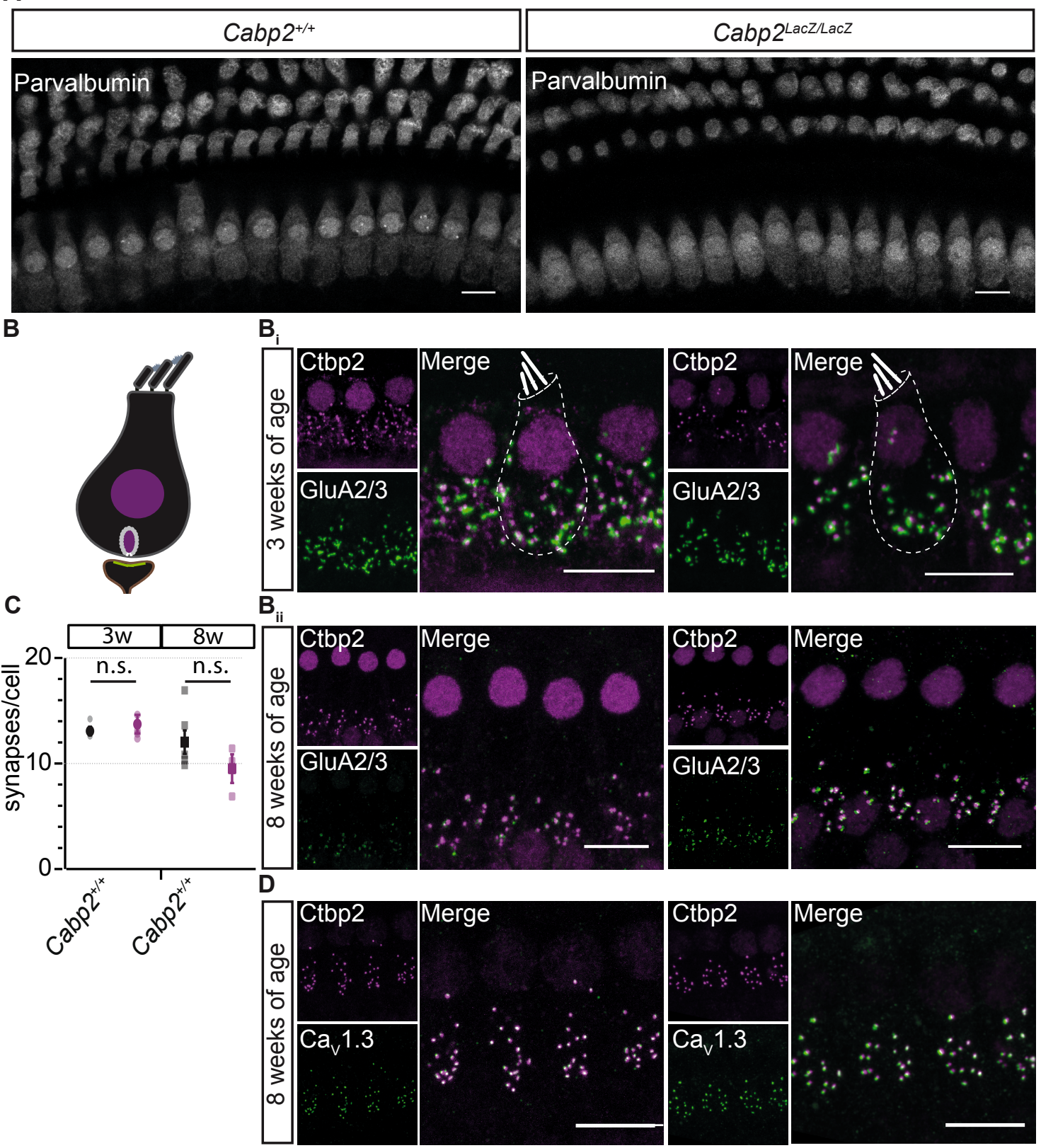

Figure 3.8: Cabp2-deficiency does not impair IHC integrity: (A) Maximal projection of apical turn of organ of Corti. IHCs and OHCs are stained with anti-parvalbumin (gray; Scale bar $=10 \mu \mathrm{m}$ ). IHCs appearance is normal in Cabp $2^{\mathrm{LaCZ} / \mathrm{LaCZ}}$ animals. (B) Schematic of immunostaining strategy. IHC nucleus and presynaptic ribbon are stained with anti-Ctbp 2 shown in magenta and the postsynaptic glutamate receptors $2 / 3$ in green. $\mathrm{B}_{i}$ Representative maximum projection of synapse staining of IHC ribbon synapses of 2- (upper) and 8-week-old animals. (lower; Scale bar $=10$ $\mu \mathrm{m})$. (C) Synapse count as synapses/cell of Cabp2 $2^{\mathrm{LaCZ} / \mathrm{LacZ}}$ and $\mathrm{Cabp} 2^{+/+} \mathrm{IHC}$ at both ages. The synapse number is normal in Cabp2 $2^{\text {LacZ/LacZ }} \mathrm{IHCs}$ at $3\left(\mathrm{Cabp}^{+/+}: \mathrm{n}=3 ; \mathrm{Cabp}^{\text {LacZ/LacZ }}: \mathrm{n}=\right.$ 4) and $8\left(\mathrm{Cabp}^{+/+}: \mathrm{n}=3 ; \mathrm{Cabp} 2^{\mathrm{LaCZ} / \mathrm{LaCZ}}: \mathrm{n}=2\right)$ weeks of age. (D) Representative immunostaining of $\mathrm{Ca}_{V} 1.3$ channels (green) and the presynaptic ribbon (magenta) showing normal $\mathrm{Ca}_{V} 1.3$ channel clustering at the active zone (Scale bar $=10 \mu \mathrm{m})$. 
at a holding potential $\left(\mathrm{V}_{h}\right)$ of $-69 \mathrm{mV}$ in young (p15-20) animals and restricted to IHCs of the mid-cochlear region $(8-12 \mathrm{kHz})$ since ABR thresholds were significantly increased from 6 to 16 kHz. The IV relationship is illustrated in Figure 3.9. $\mathrm{I}_{\mathrm{Ca}}$ IV-curves in IHCs of $\mathrm{Cabp}^{+/+}$and

A

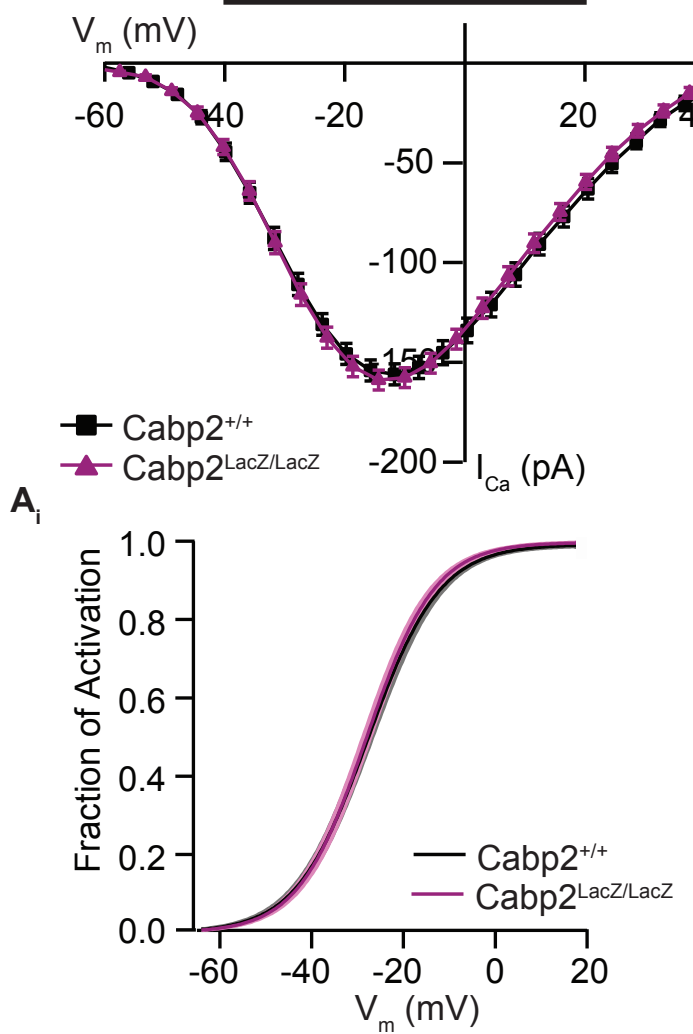

B

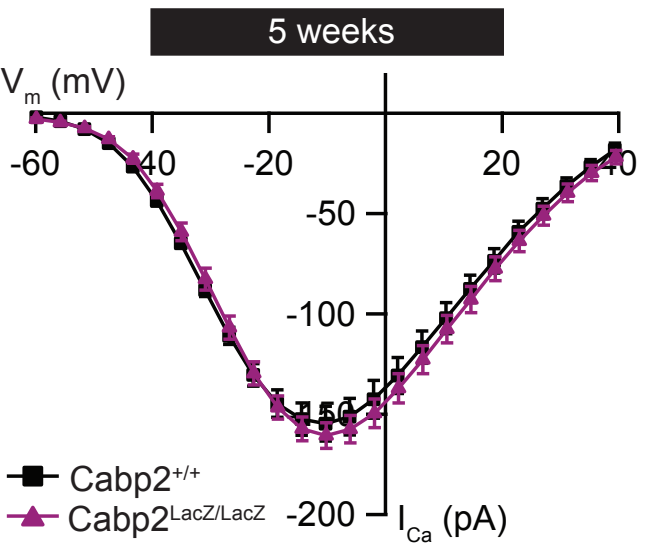

$B_{i}$

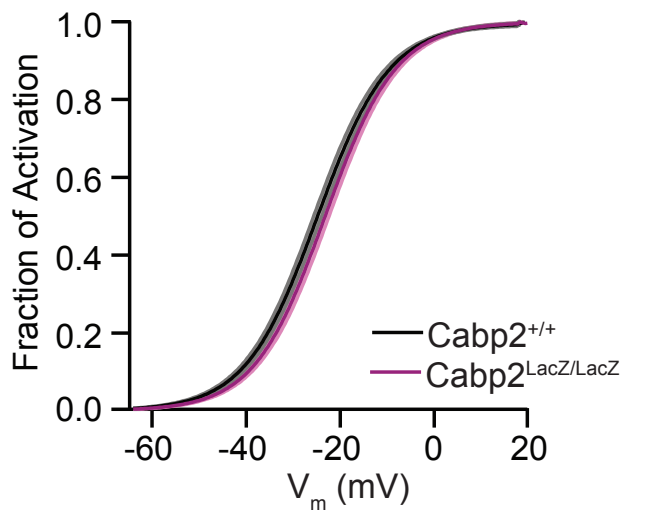

Figure 3.9: Current-voltage relationship in $\mathrm{Cabp} 2^{\mathrm{LaCZ} / \mathrm{LacZ}}$ mice: (A) Average steady-state IV for Cabp $2^{\text {LacZ/LacZ }}(\mathrm{n}=17)$ and Cabp2 $2^{+/+}(\mathrm{n}=16)$ IHCs of 2-week-old mice. $\left(\mathrm{A}_{i}\right)$ Activation curve obtained from $\mathrm{A}$. The linear portion of the IV $(0-20 \mathrm{mV})$ was divided by a line fit and fitted by a Boltzmann function. (B- $\left.\mathrm{B}_{i}\right)$ Average steady stage IV and activation curve as in $\mathrm{A}-\mathrm{A}_{i}$ but in 5week-old animals. ( $C a b p 2^{+/+}: \mathrm{n}=8 ; \operatorname{Cabp} 2^{\text {LacZ/LacZ }}: \mathrm{n}=17$ ). The IV relationship is comparable between $C a b p 2^{+/+}$and $C a b p 2^{\text {LacZ/LacZ }}$ IHCs.

Cabp2 ${ }^{\text {LacZ/LacZ }}$ animals were comparable in amplitude and $\mathrm{V}_{\text {half }}$ in p15-20 animals (Figure 3.9A, $\mathrm{A}_{i}$ ), with a significantly steeper $\mathrm{k}_{a c t}$, which was not observed when recorded from IHCs of older animals (p35-42) in the frequency range of approximately $6 \mathrm{kHz}$ (Figure 3.9B, $\mathrm{B}_{i}$; Table 3.3).

This experiment demonstrates that voltage-sensitivity of $\mathrm{Ca}_{V} 1.3 \mathrm{Ca}^{2+}$ channels in IHCs is not decreased in Cabp2 $2^{\text {LacZ/LacZ }}$ animals. Interestingly, the depolarizing shift of $\mathrm{V}_{\text {half }}$ observed in HEK293/hSK3-1 cells in the absence of CABP2 was not reproduced in IHCs. This could be due to compensation by multiple other protein interaction partners of $\mathrm{Ca}_{V} 1.3$ in IHCs. Hence, the hypothesis of reduced voltage-sensitivity due to the mutation in CABP2 recorded in HEK-cells as an underlying cause of DFNB93 can be rejected. 
Table 3.3: Biophysical properties of $\mathrm{Ca}_{V} 1.3$ in IHCs: p-values comare Cabp2 $2^{\text {LacZ/LacZ }}$ and Cabp2 $2^{+/+}$IHCs of two age groups.

\begin{tabular}{lllll}
\hline & $\mathbf{V}_{\text {half }}(\mathbf{m V})$ & $\mathbf{p}\left(\mathbf{V}_{\text {half }}\right)$ & $\mathbf{k}_{a c t}(\mathbf{m V})$ & $\mathbf{p}\left(\mathbf{k}_{a c t}\right)$ \\
\hline \hline 2 weeks & & & & \\
Cabp2 $2^{+/+}$ & $-27.72 \pm 0.94$ & & $7.41 \pm 0.12$ & \\
$\begin{array}{l}\text { Cabp2 } 2_{\text {LacZ/LacZ }} \\
\text { 5 weeks }\end{array}$ & $-28.03 \pm 0.85$ & 0.811 & $7.07 \pm 0.08$ & 0.023 \\
Cabp2 $^{+/+}$ & $-24.84 \pm 0.90$ & & & \\
Cabp2 $^{\text {LacZ/LacZ }}$ & $-26.20 \pm 1.69$ & 0.97 & $7.50 \pm 0.12$ & \\
\hline
\end{tabular}

\subsection{5 $\mathrm{Ca}_{V} 1.3 \mathrm{Ca}^{2+}$ channel inactivation is increased in $\mathrm{Cabp2}^{\mathrm{LacZ} / L a c Z}$ mice}

The second hypothesis derived from HEK293 cell experiments was that signal transmission at the IHC ribbon synapse may be impaired due to an increase in $\mathrm{Ca}_{V} 1.3 \mathrm{Ca}^{2+}$ channel steady-state inactivation. To test this hypothesis, I studied the $\mathrm{Ca}_{V} 1.3 \mathrm{Ca}^{2+}$ channel inactivation using the same age groups, frequency region, patch-configuration and $\mathrm{V}_{\text {holding }}$ as above if not stated otherwise. I applied $500 \mathrm{~ms}$ step depolarizations to the voltage of maximal $\mathrm{Ca}^{2+}$ current $\left(\mathrm{V}_{\text {Imax }}\right)$. In p15-19 animals I tested VDI and CDI by measuring both $\mathrm{I}_{C a}$ and $\mathrm{I}_{B a}$. I chose not to record them from the same cells, as washing out of $\mathrm{Ca}^{2+}$ proved to be incomplete even after prolonged perfusion. $\mathrm{I}_{C a}$ and $\mathrm{I}_{B a}$ (mean \pm s.e.m.) are presented in Figure 3.10A. $\mathrm{R}_{500}$ values were defined as residual current fraction $\left(\mathrm{I}_{\text {res }}\right)$ at $500 \mathrm{~ms}$ after normalization to the maximum current $\left(\mathrm{I}_{\max }\right)$. Under both conditions, inactivation was more pronounced in $\mathrm{Cabp} 2^{\mathrm{LacZ} / \mathrm{LacZ}}$ compared to $\mathrm{Cabp} 2^{+/+} \mathrm{IHCs}$. The extent of $\mathrm{I}_{C a}$ and $\mathrm{I}_{B a}$ was already decreased within the first $100 \mathrm{~ms}$ and was statistically different after 500 ms (Figure 3.10B; $\mathrm{I}_{C a}: \mathrm{p}<0.001, \mathrm{I}_{B a}: \mathrm{p}=0.002$; tests: Wilcoxon rank sum test).

While the effect of VDI proved to be statistically significant ( $p=0.003$, Student's t-test), CDI only showed a non-significant trend towards an increase ( $p>0.5$, Wilcoxon rank sum test). This data suggest that in the absence of Cabp2 VDI, but not CDI of IHC $\mathrm{Ca}_{V} 1.3$ is significantly enhanced.

To further elucidate the cause for the observed progression of hearing impairment, I probed whether inactivation is greater in IHCs at higher age (p35-42). In 5-week-old animals only $\mathrm{Ca}^{2+}$ current inactivation summarizing CDI and VDI was recorded (Figure 3.10C). $\mathrm{R}_{500}$ was significantly decreased in $C a b p 2^{\text {LacZ/LacZ }}\left(\mathrm{R}_{500}: 0.55 \pm 0.04\right)$ compared to Cabp2 $2^{+/+}$IHCs $\left(\mathrm{R}_{500}\right.$ : $0.68 \pm 0.05 ; \mathrm{p}<0.001$, Wilcoxon rank sum test). Furthermore, at higher age Cabp2 $2^{\text {LacZ/LacZ }}$ IHCs displayed less total inactivation at $\mathrm{R}_{500}$ compared to p15-20 Cabp2 $2^{\mathrm{LacZ} / \mathrm{LacZ}}$ animals $\left(\mathrm{R}_{500}\right.$ : 0.61 vs. $0.55, \mathrm{p}=0.046$, Wilcoxon rank sum test ). An age-dependent difference in the extent 

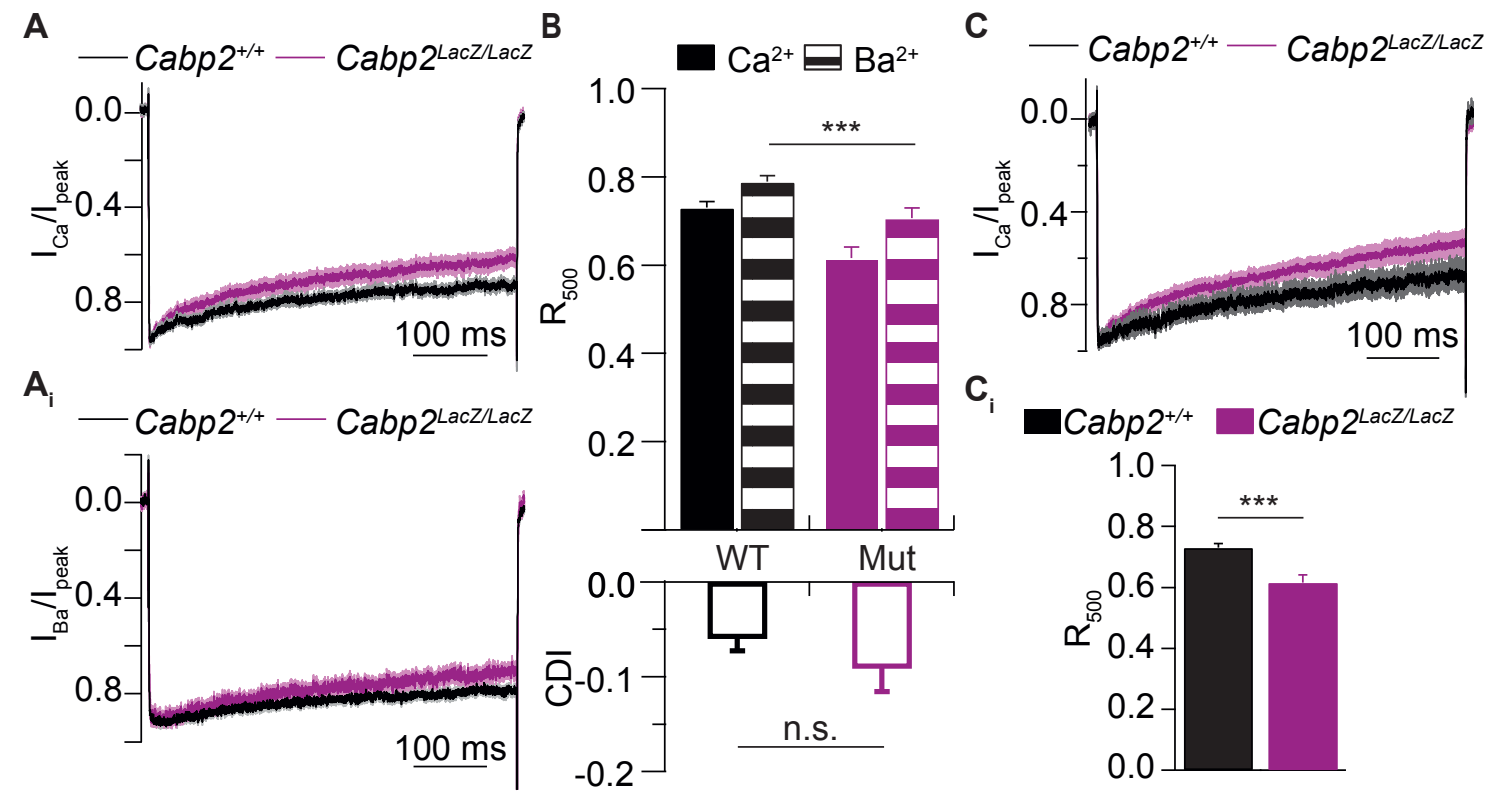

Figure 3.10: $C a b p 2^{\mathrm{LacZ} / \mathrm{LacZ}}$ IHC show increased $\mathrm{Ca}_{V} 1.3$ inactivation: (A) Normalized average $\mathrm{I}_{C a}$ traces upon $500 \mathrm{~ms}$ step depolarizations of $\mathrm{Cabp}^{\mathrm{LaCZ} / \mathrm{LaCZ}}(\mathrm{n}=16)$ and $\mathrm{Cabp}^{+/+}(\mathrm{n}=15)$ IHCs (mean \pm s.e.m.). $\left(\mathrm{A}_{i}\right)$ Same experimental paradigm as in $\mathrm{A}$, but with $\mathrm{Ba}^{2+}$ as charge carrier $\left(C_{a b p 2^{+/+}}: \mathrm{n}=29, C_{a b p} 2^{\text {LacZ/LacZ }}: \mathrm{n}=18\right)$. Inactivation of $\mathrm{I}_{C a}$ and $\mathrm{I}_{B a}$ are both more pronounced upon Cabp2-deficiency. (B) $\mathrm{R}_{500}$-values derived from $\mathrm{A}$ of $\mathrm{Ca}^{2+}$ and $\mathrm{Ba}^{2+}$ currents. VDI is induced by $\mathrm{Ba}^{2+}$ itself and is significantly increased in Cabp $2^{\mathrm{LacZ} / \mathrm{LacZ}} \mathrm{IHCs}$. CDI tended to be increased in $C a b p 2^{\text {LacZ/LacZ }}$ IHC. (C) Normalized average $\mathrm{I}_{C a}$ inactivation traces of p35-42 animals for Cabp2 $2^{\text {LacZ/LacZ }}(\mathrm{n}=17)$ and Cabp2 $2^{+/+} \mathrm{IHCs}(\mathrm{n}=8) .\left(\mathrm{C}_{i}\right) \mathrm{R}_{5} 00$ values of C. Note that inactivation is significantly increased in $\mathrm{Cabp} 2^{\mathrm{LacZ} / \mathrm{LacZ}} \mathrm{IHC}$.

of $\mathrm{I}_{\mathrm{Ca}}$ inactivation was not observed in $\mathrm{Cabp}^{+/+}$IHCs. This data suggests, that $\mathrm{Ca}^{2+}$ channel inactivation under these recording conditions is not increasing with age in Cabp $2^{\text {LacZ/LacZ }}$ IHCs.

Table 3.4: Inactivation in Cabp2 $2^{+/+}$and Cabp2 $2^{\text {LacZ/LacZ }}$ IHCs of young p15-20 animals

\begin{tabular}{cclc}
\hline VDI & $p_{V D I}$ & CDI & $p_{C D I}$ \\
\hline \hline$C_{C a b p 2^{+/+}}-0.21 \pm 0.01$ & & $-0.06 \pm 0.01$ & \\
$C^{\text {Labp }}{ }^{\text {LacZ/LacZ }}-0.29 \pm 0.03$ & 0.003 & $-0.09 \pm 0.03$ & 0.85 \\
\hline
\end{tabular}

$\mathrm{Ca}_{V} 1.3 \mathrm{Ca}^{2+}$ channel inactivation was further examined by applying a train stimulation protocol in p15-20 IHCs, which more closely resembles in vivo stimulation. 60 consecutive 10 ms pulses were applied with $5 \mathrm{~ms}$ gaps as illustrated in Figure $3.11 \mathrm{~A}$ and $\mathrm{I}_{C a}$ were recorded. $\mathrm{Ca}^{2+}$ channel inactivation was calculated by normalizing the mean of the last $5 \mathrm{~ms}$ of the respective pulse $\mathrm{n}\left(\mathrm{I}_{n}\right)$ to the mean $5 \mathrm{~ms}$ of the first pulse $\left(\mathrm{I}_{1 s t}\right)$. Assuming that $\mathrm{V}_{\text {holding }}$ of the clamped cell would determine the fraction of steady-state inactivated $\mathrm{Ca}^{2+}$ channels at resting conditions, 
the train stimuli were applied at 2 different holding potentials. Before each recording, the cells were left at this new $\mathrm{V}_{\text {holding }}$ for at least $2 \mathrm{~min}$. The following $\mathrm{V}_{\text {holding }}$ were chosen: $-69 \mathrm{mV}$, the assumed resting potential of apical coil IHC at room temperature (Johnson et al., 2011) and -49 $\mathrm{mV}$, the voltage at which a higher fraction of $\mathrm{Ca}_{V} 1.3$ channels is in open state. Normalized train responses are illustrated in Figure 3.11B. $\mathrm{I}_{C a}$ traces were normalized to $\mathrm{I}_{C a}$ of the first pulse. At the onset and end of the train the mean of 5 consecutive pulses was calculated and termed onset and sustained inactivation. In Cabp $2^{\text {LacZ/LacZ }}$ IHCs rested at $-69 \mathrm{mV}$ both, onset and sustained inactivation were significantly enhanced. The normalized train currents were then fitted by a double exponential function of the following form: $y_{0}+A_{1} * \exp \left(-\left(x-x_{0}\right) / \tau_{1}\right)+A_{2} * \exp \left(-\left(x-x_{0}\right) / \tau_{2}\right)$, which showed that the current amplitudes at onset and the end of the train were both significantly reduced in Cabp2 $2^{\text {LacZ/LacZ }}$ IHCs (Values for train onset and end: $\mathrm{p}<0.0001$; fit-amplitude: fast and slow: $\mathrm{p}=0.006$, Student's t-test; Figure 3.11B). Train responses at $-49 \mathrm{mV}$ resulted in more comparable onset currents between $\mathrm{Cabp} 2^{+/+}$and Cabp $2^{\mathrm{LacZ} / \mathrm{LacZ}} \mathrm{IHC}$, only the current amplitudes at the end of the train were reduced in Cabp $2^{\text {LacZ/LacZ }}$ IHCs (Figure $3.11 \mathrm{~B}_{i} ; \mathrm{p}=0.003$, Wilcoxon rank sum test).

After identifying a continuous increase of $\mathrm{Ca}_{V} 1.3$ channel inactivation upon ongoing stimulation the question arose, what time the $\mathrm{Ca}^{2+}$ channels needed to fully recover from inactivation. To measure recovery from inactivation a double pulse (P1 and P2) was applied to open a smaller number of channels ( $10 \mathrm{~ms}$ to $-25 \mathrm{mV}$ ) separated by a $200 \mathrm{~ms}$ conditioning or inactivating pulse to $\mathrm{V}_{\text {Imax }}$ to affect a maximum fraction of $\mathrm{Ca}_{V} 1.3$ channels. The time interval between conditioning and post pulse was prolonged with every sweep and the ratio of the $\mathrm{Ca}^{2+}$ current between post(P2) and prepulse (P1) compared in respect to $\Delta \mathrm{t}$ (for the protocol see Figure 3.11C). In Figure $3.11 \mathrm{D}, \mathrm{D}_{i}$ average CDI recovery traces are presented, for Cabp $2^{\text {LacZ/LacZ }}$ and $\mathrm{Cabp} 2^{+/+} \mathrm{IHCs}$. The results show that $\mathrm{V}_{\text {holding }}$ influences recovery behavior of $\mathrm{I}_{C a}$ in Cabp2 $2^{\text {LacZ/LacZ }}$ IHCs. After $1 \mathrm{~s}$ recovery times $\mathrm{P} 2 / \mathrm{P} 1$ ratios were comparable between both genotypes when kept at $-84 \mathrm{mV}$ (p15-20 animals, Figure 3.11G) while P2/P1 ratio of Cabp2 deficient IHCs kept at -69 mV lagged behind wild-type by $25 \%$ at the same time intervall. While these data cannot be directly compared, since they were obtained at two different ages, they suggest that $\mathrm{V}_{\text {holding }}$ impairs recovery from inactivation in Cabp $2^{\mathrm{LacZ} / \mathrm{LacZ}} \mathrm{IHCs.}$

Taken together, Cabp2 $2^{\text {LacZ/LacZ }}$ IHCs have more pronounced $\mathrm{Ca}_{V} 1.3$ inactivation. Interestingly, this difference in inactivation is mainly due to a difference in VDI, whereas CDI does not seem to be significantly affected by the deficiency of Cabp2. Upon ongoing stimulation $\mathrm{Ca}_{V} 1.3$ channels in Cabp2 $2^{\mathrm{LaCZ} / \mathrm{LacZ}}$ IHC showed more inactivation at stimulus onset and inactivation increased till the end of the train. Unexpectedly, by changing $\mathrm{V}_{\text {holding }}$ to depolarized potentials ($49 \mathrm{mV}$ ) the inactivation difference at train onset was not enlarged, but rather diminished. Finally, recovery from inactivation is impaired in Cabp $2^{\mathrm{LacZ} / \mathrm{LacZ}}$ IHCs and likely dependent on IHC re- 
A

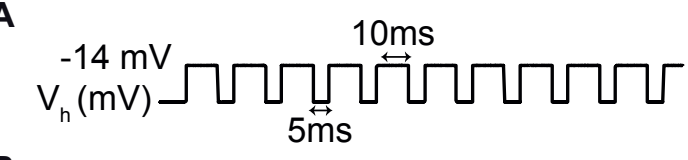

B
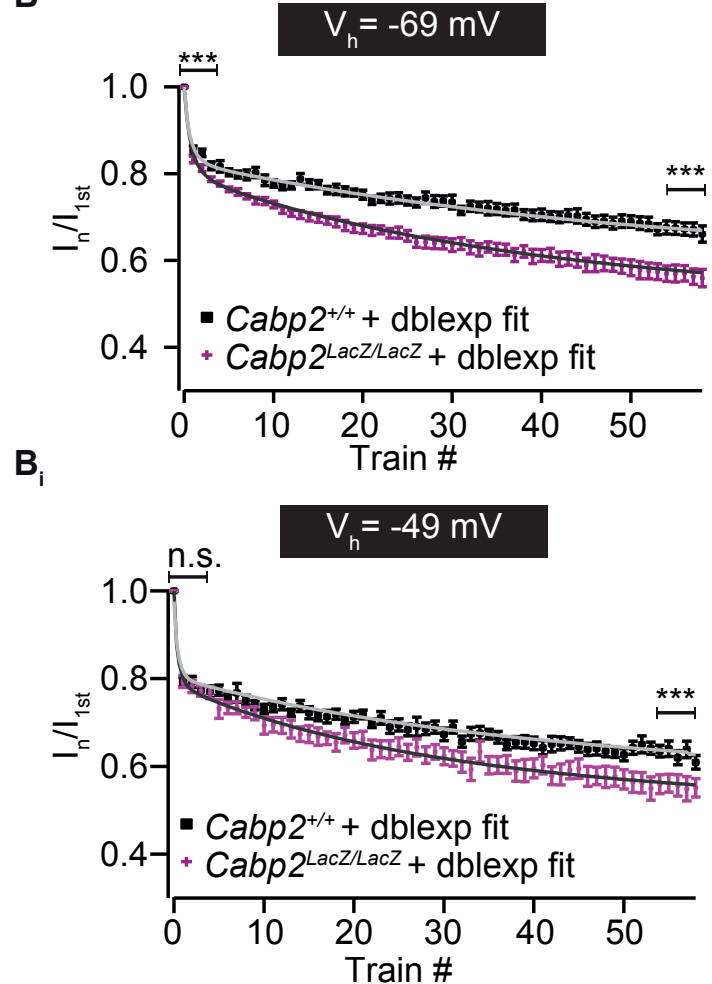

C
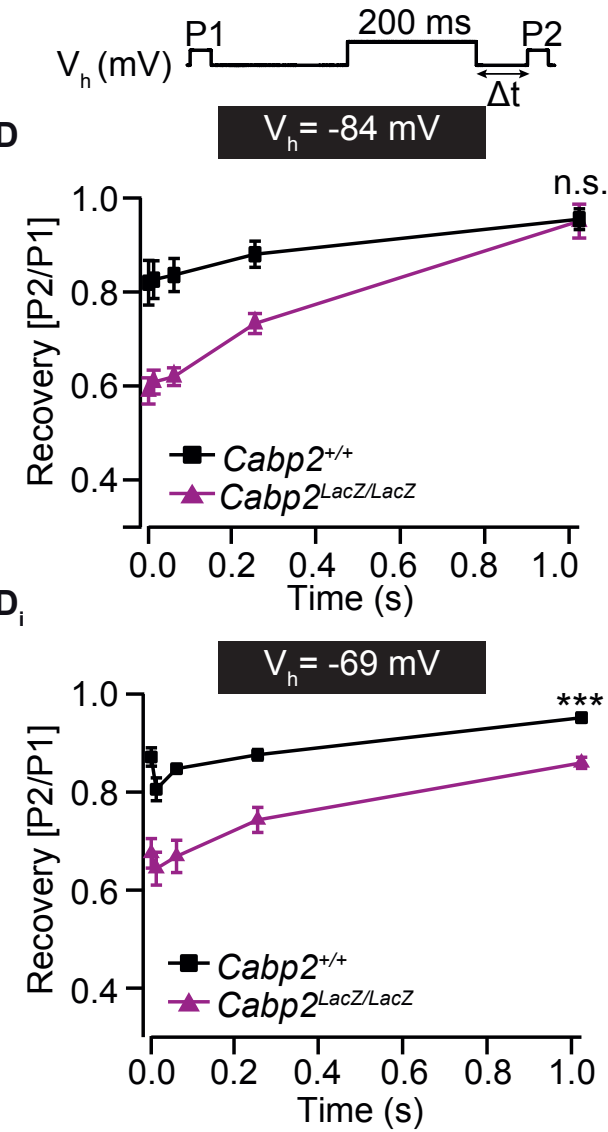

Figure 3.11: Recovery from inactivation is impaired in Cabp2 $2^{\text {LacZ/LacZ }}$ IHCs: (A) Detailed schematic of the train stimulus enlarging 10 of the 60 pulses applied to record inactivation responses to consecutive pulse stimulation. (B) Average train responses of $\mathrm{Cabp}^{+/+}(\mathrm{n}=14)$ and Cabp2 $2^{\text {LacZ/LacZ }}(\mathrm{n}=14)$ IHCs at $\mathrm{V}_{\text {holding }}$ of $-69 \mathrm{mV}$ and at $-49 \mathrm{mV}\left(\mathrm{B}_{i}\right)$. Average double exponential fits are highlighted as gray superimposed lines of the respective trace. At $\mathrm{V}_{\text {holding }}$ of $-69 \mathrm{mV}$ onset and sustained responses are significantly reduced, while at $\mathrm{V}_{\text {holding }}$ of $-49 \mathrm{mV}$ only sustained currents are significantly affected in Cabp $2^{\text {LacZ/LacZ }} \mathrm{IHCs}$ compared to Cabp $2^{+/+}$ IHCs. (C) Schematic of double pulse protocol to record recovery from inactivation: P1 shows the pre- and P2 the post-pulse depolarization to $-25 \mathrm{mV}$ for $10 \mathrm{~ms}$. In every sweep the time interval $(\Delta \mathrm{t})$ was prolonged (4-16-64-256-1024 ms) between the $200 \mathrm{~ms}$ conditioning/inactivating pulse to $\mathrm{V}_{\text {Imax }}$ and P2. (D) $\mathrm{V}_{\text {holding }}$ of $-84 \mathrm{mV}$ : Average P2:P1 ratio was plotted as a function of time interval recorded from p15-20 Cabp2 $2^{+/+}(\mathrm{n}=11)$ and Cabp2 $2^{\mathrm{LacZ} / \mathrm{LacZ}}(\mathrm{n}=7)$ IHCs. Note that $\mathrm{I}_{C a}$ recovers after $1 \mathrm{~s}$ in Cabp2 ${ }^{\text {LacZ/LacZ }}$ IHCs. $\left(\mathrm{D}_{i}\right) \mathrm{V}_{\text {holding }}$ of $-69 \mathrm{mV}$ : Average recovery traces of $\mathrm{Cabp}^{+/+}(\mathrm{n}=9)$ and Cabp2 $2^{\text {LacZ/LacZ }}(\mathrm{n}=8)$ IHCs recorded from 5-week-old animals. Recovery time of $\mathrm{I}_{C a}$ exceeds $1 \mathrm{~s}$ in Cabp2 $2^{\text {LacZ/LacZ }}$ compared to Cabp2 $2^{+/+}$IHCs $(\mathrm{p}<0.0001$, Student's t-test). 
ceptor potential. Together these results well support the second hypothesis suggesting increased steady-state inactivation of IHC Ca $1.3 \mathrm{Ca}^{2+}$ channels as the underlying cause for DFNB93.

\subsubsection{Cabp2-deficiency results in increased sustained exocytosis}

So far, I have presented evidence for an increased steady-state inactivation as a candidate mechanism for DFNB93. However, how $\mathrm{Ca}^{2+}$ channel inactivation influences synaptic transmission, i.e. presynaptic vesicle release, has not been addressed yet. Therefore I performed membrane capacitance $\left(\mathrm{C}_{m}\right)$ recordings in IHCs of young (p15-20) and 5-week-old animals. To record IHC $\mathrm{C}_{m}$, a sine wave of $35 \mathrm{mV}$ peak-to-peak amplitude was applied before and after a short depolarization window of incrementing duration (for representative traces see Figure 3.12A). The mean $\Delta \mathrm{C}_{m}$ was derived by subtracting the capacitance of the cell at rest (before depolarization) from $\mathrm{C}_{m} 100 \mathrm{~ms}$ after stimulation (Metod see (Moser and Beutner, 2000)). Figure 3.12 $\mathrm{A}_{i}$ upper summarizes the mean $\Delta \mathrm{C}_{m}$ in response to depolarizing pulses of increasing duration for p15-20 animals with the respective integrated $\mathrm{Ca}^{2+}$ current $\left(\mathrm{Ca}^{2+}\right.$ charge: $\mathrm{Q}_{C a}$; Figure $3.12 \mathrm{~A}_{i}$ lower). Cells were depolarized for: 5 - $10-20-50-100-200$ and $500 \mathrm{~ms}$. Depolarization of $0.05-20 \mathrm{~ms}$ is believed to primarily trigger fusion of readily releasable vesicles (readily releasable pool (RRP): (Moser and Beutner, 2000)) and capacitance increments triggered after depolarizations ranging from 20 to 500 ms are thought to report the replenishment of vesicles into the RRP and their subsequent fusion. In IHC of young Cabp $2^{\mathrm{LacZ} / \mathrm{LacZ}}$ animals, I observed a weak tendency towards reduced sustained exocytosis in Cabp2 $2^{\mathrm{LacZ} / \mathrm{LacZ}} \mathrm{IHC}$ (> $20 \mathrm{~ms}$ depolarization) when the cells were clamped to -69, which was not found at $-84 \mathrm{mV}$ (Figure $3.12 \mathrm{~A}_{i}$ ). In addition to $\Delta \mathrm{C}_{m}$ the respective $\mathrm{Ca}^{2+}$ charge $\left(\mathrm{Q}_{C a}\right)$ was monitored (Figure 3.12 $\mathrm{A}_{i}$ lower). Due to a stronger $\mathrm{Ca}^{2+}$ channel inactivation $\mathrm{Q}_{C a}$ was significantly reduced in mutant IHCs for longer pulses ( 200 and $500 \mathrm{~ms}$, respectively; $\mathrm{V}_{\text {holding }}=69$ $\mathrm{mV}: \mathrm{p}_{200}=0.022$, Student's t-test; $\mathrm{p}_{500}=0.045$, Student's t-test). Interestingly, a tendency towards reduced exocytosis was not observed in 5-week-old Cabp2 $2^{\text {LacZ } / L a c Z}$ animals (Figure 3.12B B $_{i}$, that actually showed the opposite. There, sustained vesicle fusion was significantly elevated $\left(\mathrm{p}_{50}=\right.$ $0.041 ; \mathrm{p}_{100}=0.0012 ; \mathrm{p}_{200}=0.003$, Wilcoxon rank sum tests). This phenomenon seems counter intuitive at the first sight, since an increase in $\mathrm{Ca}^{2+}$ channel inactivation would be expected to result in reduced sustained exocytosis. One plausible explanation for the increase in sustained exocytosis in 5-week-old animals might be an impairment of $\mathrm{Ca}^{2+}$ buffering at the IHC active zone in the absence of Cabp2, which would allow elevated $\mathrm{Ca}^{2+}$ spread. Such spread of $\mathrm{Ca}^{2+}$ could trigger exocytosis outside the AZs, as proposed in a recent study, investigating the role of other EF-hand $\mathrm{Ca}^{2+}$ binding proteins in IHCs (Pangršič et al., 2015).

In summary, $\Delta \mathrm{C}_{m}$ recordings in IHC of p15-18 IHCs revealed modestly decreased sustained exocytosis upon the mutation of Cabp2. However, the opposite effect was observed in Cabp2 $2^{\text {LacZ/LacZ }}$ IHCs of older mice, where sustained exocytosis was approximately 1.7 fold increased. $\mathrm{As} \mathrm{Ca}^{2+}$ current 
A

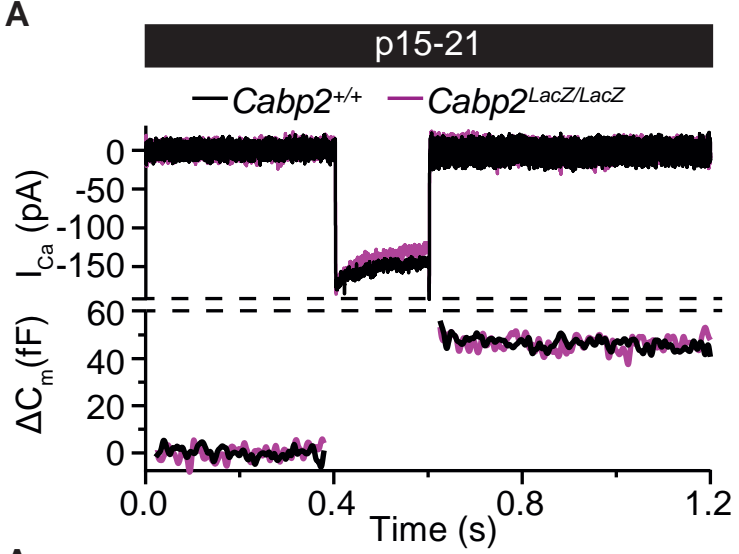

$A_{i}$

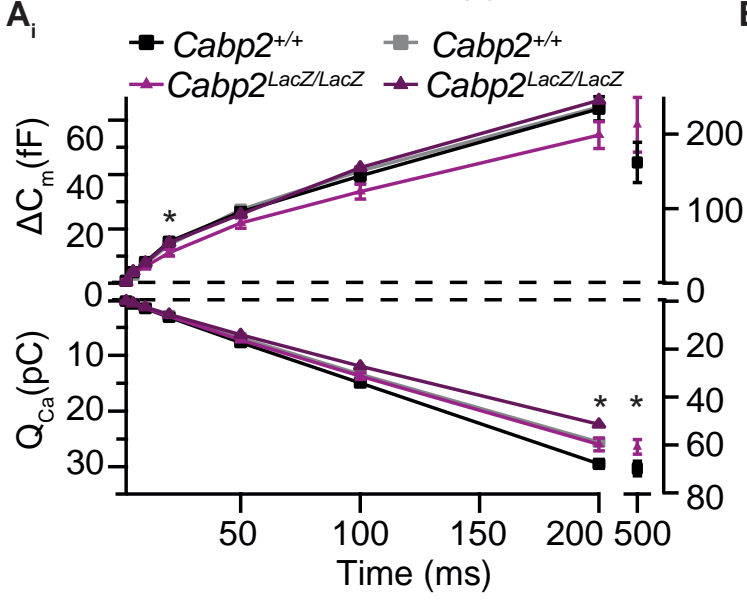

B

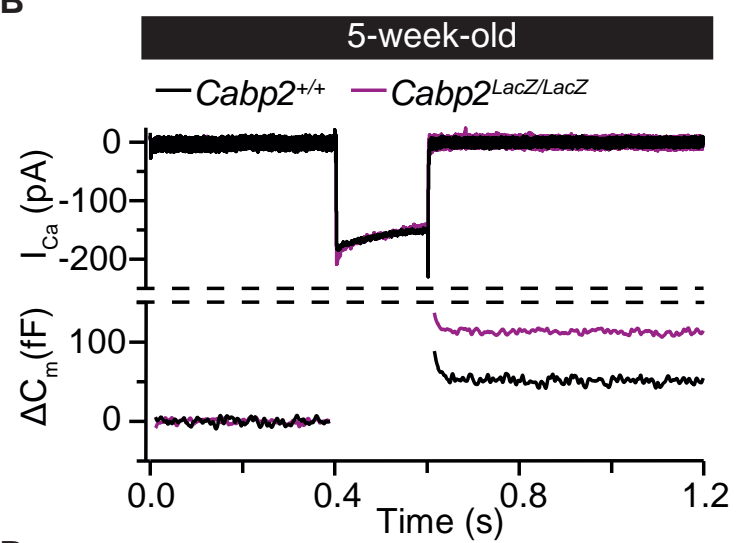

$B_{i}$

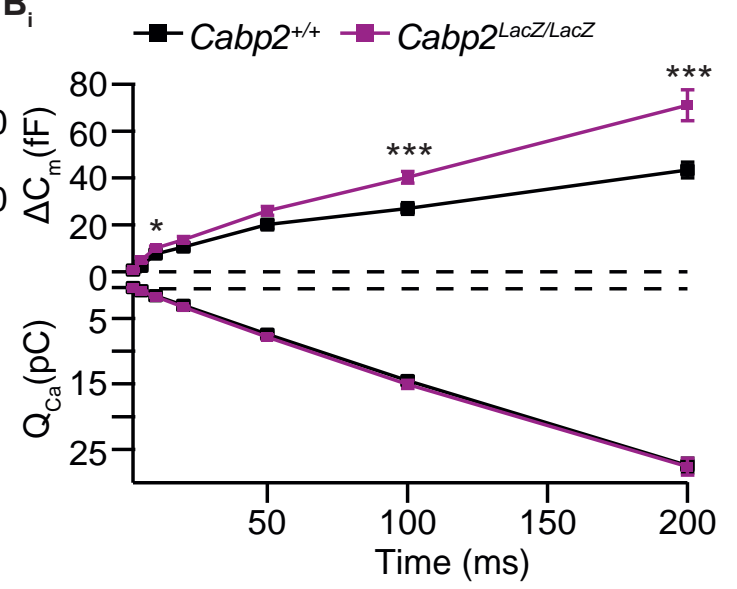

Figure 3.12: Cabp2-deficiency affects exocytosis of young (p14-20) and older (p35-42) animals in opposite ways: (A) Capacitance recordings from p15-20 animals: Representative $\mathrm{I}_{C a}$ (upper) and respective $\Delta \mathrm{C}_{m}$ (lower) responses of Cabp2 $2^{+/+}$and Cabp $2^{\text {LacZ/LacZ }}$ IHCs upon 200 ms depolarization. $\mathrm{A}_{i}$ Traces were normalized to the respective cell capacitance. Mean \pm s.e.m. traces of IHC held either at $-84 \mathrm{mV}\left(\operatorname{Cabp}^{+/+}: \mathrm{n}=8, \operatorname{Cabp} 2^{\text {LacZ/LacZ }}: \mathrm{n}=5\right)$ or $-69 \mathrm{mV}\left(\operatorname{Cabp}^{+/+}\right.$: $\mathrm{n}=12, \operatorname{Cabp} 2^{\text {LacZ } / \text { LacZ }}: \mathrm{n}=16$ ). Note that $\Delta \mathrm{C}_{m}$ is significantly reduced for $50 \mathrm{~ms}$ depolarizations affecting sustained release $(\mathrm{p}<0.05$, Student's t-test) followed by a trend toward reduced sustained exocytosis in IHCs kept at $-69 \mathrm{mV}$. This trend is absent by changing $\mathrm{V}_{\text {holding }}$ to $-84 \mathrm{mV}$ (no statistical test due to the low n). (B) IHC capacitance recordings from 5-week-old animals: Representative capacitance and current traces of $\mathrm{Cabp}^{+/+}$and $\mathrm{Cabp} 2^{\mathrm{LacZ} / \mathrm{LacZ}}$ IHCs illustrating enhanced $\Delta \mathrm{C}_{m}$ in a Cabp2 $2^{\text {LacZ/LacZ }}$ IHC compared to control. $\left(\mathrm{B}_{i}\right)$ Mean \pm s.e.m. $\Delta \mathrm{C}_{m}$ and respective $\mathrm{Q}_{C a}$ recordings of Cabp2 ${ }^{\text {LacZ/LacZ }}(\mathrm{n}=14)$ and $C a b p 2^{+/+}(\mathrm{n}=8)$ IHCs. $\Delta \mathrm{C}_{m}$ is increased for 10-100-200 ms depolarization durations $(\mathrm{p}=0.041 ; \mathrm{p}=0.001, \mathrm{p}=0.003$; tests: Wilcoxon rank sum test).

integrals were not large in mutant IHCs, $\mathrm{Ca}^{2+}$-release efficiency is apparently increased in IHCs of older animals. One plausible explanation for increased $\mathrm{Ca}^{2+}$-release efficiency is a higher $\mathrm{Ca}^{2+}$ spread due to the lack of Cabp2. For this, one would need to assume that Cabp2 might also act as a $\mathrm{Ca}^{2+}$ buffer. The fact that sustained exocytosis in IHCs of young Cabp2 $2^{\text {LacZ/LacZ }}$ 
animals was slightly reduced might reflect reduced synaptic exocytosis due to decreased synaptic $\mathrm{Ca}^{2+}$ influx, which was masked less by extrasynaptic exocytosis. The age-dependent change observed in capacitance measurements suggests that $\mathrm{Cabp} 2$ plays a greater role in $\mathrm{IHC} \mathrm{Ca}^{2+}$ buffering at higher age e.g. due to a higher expression level.

\subsubsection{Cabp2-deficiency impairs $\mathrm{Ca}^{2+}$ buffering in IHCs}

The hypothesis that Cabp2 contributes to $\mathrm{Ca}^{2+}$ buffer in IHCs, therefore prevents extrasynaptic vesicle release was tested by three experimental approaches that build on previous work in our laboratory on mice lacking the EF-hand binding proteins calbindin-28k, calretinin and parvalbumin- $\alpha$ (Pangršič et al., 2015):

1. IHC perforated-patch recording to investigate exocytosis upon prolonged stimulation in both genotypes as excess sustained exocytosis can reflect extrasynaptic release.

2. IHC ruptured-patch experiments with application of synthetic $\mathrm{Ca}^{2+}$ chelators to measure sustained exocytosis under comparable intracellular $\mathrm{Ca}^{2+}$ buffer conditions in Cabp2 $2^{\mathrm{LacZ} / \mathrm{LacZ}}$ and $C a b p 2^{+/+}$control IHCs.

3. Extracellular recordings of spiking activity in single spiral ganglion neurons as a readout for exocytosis at the AZ of IHCs.

The first set of experiments addressed the question of $\mathrm{C}_{m}$ increments in response to repetitive stimulation. I applied 10 consecutive pulses of $100 \mathrm{~ms}$ each followed by $200 \mathrm{~ms}$ capacitance recordings. These recordings were performed in perforated-patch configuration in IHCs of 5weeks-old animals. Figure 3.13A demonstrates a representative $\mathrm{I}_{C a}$ and $\mathrm{C}_{m}$ trace (Figure 3.13A, $\mathrm{A}_{i}$ ) of Cabp2 $2^{+/+}$and Cabp2 $2^{\text {LacZ/LacZ }}$ IHCs. Figure 3.13B, $\mathrm{B}_{i}$ summarizes $\Delta \mathrm{C}_{m}$ and respective $\mathrm{Q}_{C a}$ for both genotypes showing a transient depression in $\Delta \mathrm{C}_{m}$ followed by enhanced vesicle fusion in Cabp2 $2^{\mathrm{LacZ} / \mathrm{LacZ}} \mathrm{IHCs}$, while $\Delta \mathrm{C}_{m}$ remains constant in Cabp2 $2^{+/+}$controls throughout the train. $\Delta \mathrm{C}_{m}$ was approximately doubled in Cabp2 $2^{\mathrm{LacZ} / \mathrm{LacZ}} \mathrm{IHC}$ for the $10^{\text {th }}$ pulse while $\mathrm{Q}_{C a}$ was reduced by approximately $25 \%$ (Figure 3.13B,C). The steady increase in exocytosis despite the reduced $\mathrm{Ca}^{2+}$ influx is consistent with impaired $\mathrm{Ca}^{2+}$ buffering in Cabp2 deficient IHCs.

To further test the $\mathrm{Ca}^{2+}$ buffer hypothesis I used ruptured-patch experiments aiming to washout endogenous $\mathrm{Ca}^{2+}$ buffers and introducing the same concentration of synthetic $\mathrm{Ca}^{2+}$ buffers in IHCs of both genotypes. The intracellular solution contained $0.5 \mathrm{mM}$ of the "fast-binding" $\mathrm{Ca}^{2+}$ chelator BAPTA and $0.5 \mathrm{mM}$ of the "slow-binding" $\mathrm{Ca}^{2+}$ chelator EGTA, which our lab previously indicated to approximately mimic endogenous $\mathrm{IHC} \mathrm{Ca}{ }^{2+}$ buffer concentrations (Pangršič et al., 2015). To ensure sufficient diffusional exchange between the cell and the pipette, I waited > 5 min after establishing ruptured-patch configuration for wash-out of the relatively small and mobile $\mathrm{Ca}^{2+}$ buffers with a molecular weight of $<30 \mathrm{kDa}(\mathrm{Cabp} 2: 21 \mathrm{kDa}$, calbindin-28k: $28 \mathrm{kDa}$, 
A

D

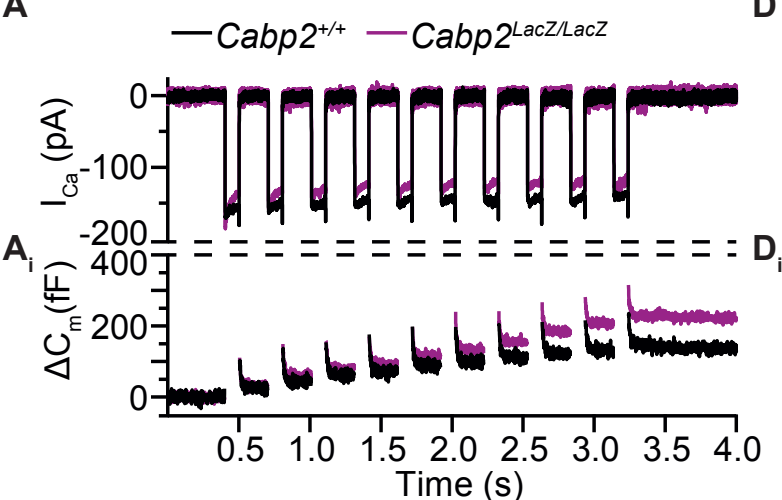

B

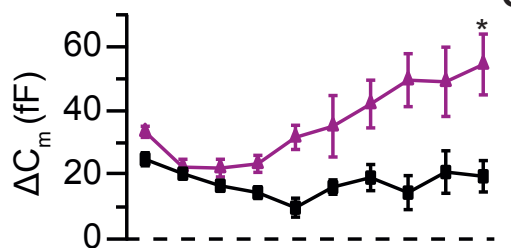

$B_{i}$

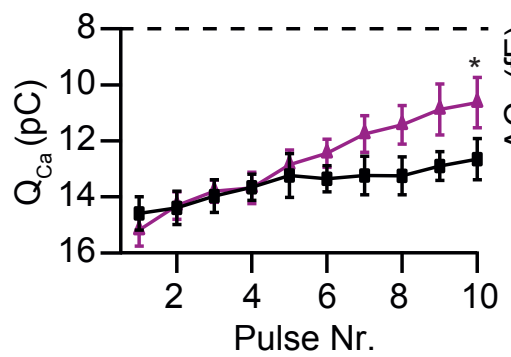

C

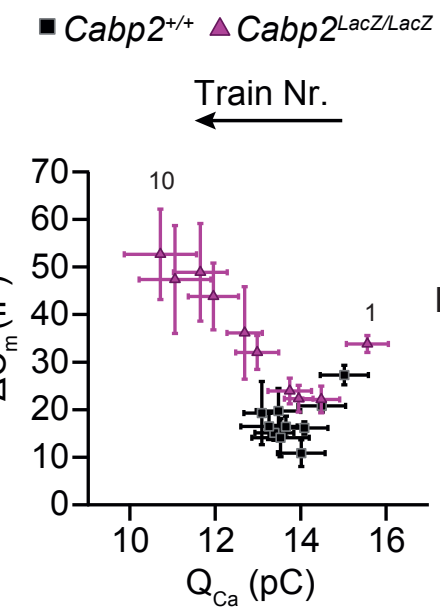

- Cabp2 $2^{+/+}-C^{2} a b p 2^{\text {LacZ/LacZ }}$

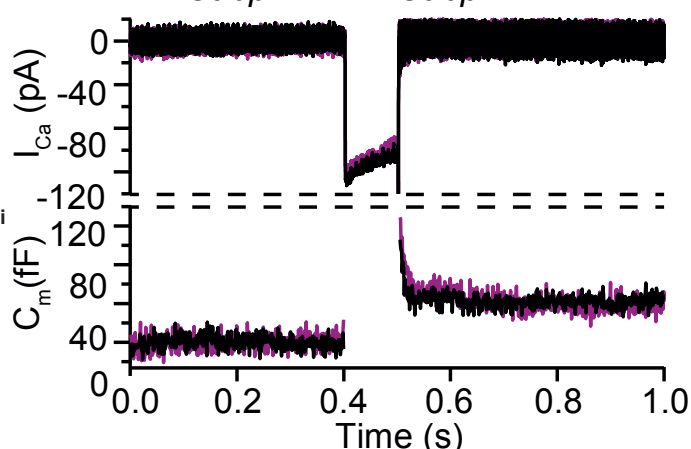

$E$

- Cabp2 $^{+/+}$- Cabp2 $2^{\text {LacZlLacZ }}$

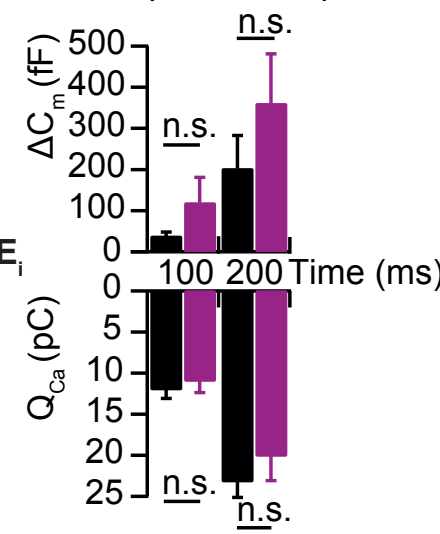

Figure 3.13: Cabp2 supports $\mathrm{Ca}^{2+}$ buffering in IHCs: (A) Representative train-response traces of $\mathrm{Cabp2}^{+/+}$and $\mathrm{Cabp} 2^{\mathrm{LacZ} / \mathrm{LacZ}}$ recordings elicited by 10 consecutive $100 \mathrm{~ms}$ pulses. $\left(\mathrm{B}, \mathrm{B}_{i}\right) \mathrm{Av}$ erage $\Delta \mathrm{C}_{m}$ and respective $\mathrm{Q}_{C a}$ traces summarizing Cabp2 $2^{+/+}(\mathrm{n}=5)$ and $\operatorname{Cabp} 2^{\text {LacZ/LacZ }}(\mathrm{n}=$ 8) recordings. Cabp2 $2^{+/+}$responses are comparable between pulses for $\Delta \mathrm{C}_{m}$ and show a mild decline in $\mathrm{Q}_{C a}$. Cabp2 $2^{\mathrm{LacZ} / \mathrm{LaCZ}}$ IHCs respond with a continuous decrease in $\mathrm{Q}_{C a}$ compared to Cabp $2^{+/+}$( $\mathrm{p}=0.011$, Student's t-test), while $\Delta \mathrm{C}_{m}$ decreases within the first four pulses and then exceeds even the first response ( $\mathrm{p}=0.018$, Student's t-test). (C) $\mathrm{Ca}^{2+}$ efficiency of release displayed as average $\Delta \mathrm{C}_{m}$ vs. $\mathrm{Q}_{C a}$ derived from $\mathrm{B}$. While $\mathrm{Ca}^{2+}$ efficiency of release is rather constant in $\mathrm{Cabp2}^{+/+}$upon train stimulation, $\mathrm{Cabp} 2^{\mathrm{LacZ} / \mathrm{LacZ}}$ IHCs show increased release-efficiency with ongoing depolarizations. (D, $\mathrm{D}_{i}$ ) Representative ruptured-patch $\Delta \mathrm{C}_{m}$ and $\mathrm{Q}_{C a}$ traces for Cabp2 $2^{+/+}$ and Cabp2 $2^{\text {LacZ/LacZ }}$ IHCs. (E, $\left.\mathrm{E}_{i}\right)$ Summary of Cabp2 $2^{+/+}(\mathrm{n}=9)$ and Cabp2 $2^{\text {LacZ/LacZ }}(\mathrm{n}=9)$ recordings obtained under controlled buffer conditions. Note comparable $\Delta \mathrm{C}_{m}$ and $\mathrm{Ca}^{2+}$ charge for 100 and $200 \mathrm{~ms}$ pulses.

parvalbumin- $\alpha$ : 9-11 kDa) and appropriate introduction of BAPTA and EGTA into the cells. After such waiting periods, sustained exocytosis was recorded in response to 100 and $200 \mathrm{~ms}$ depolarizing stimuli. Figure 3.13D illustrates an exemplary $\mathrm{I}_{C a}$ and $\Delta \mathrm{C}_{m}$ trace for $100 \mathrm{~ms}$ depolarization pulses which were comparable between $C a b p 2^{\text {LacZ/LacZ }}$ and Cabp2 $2^{+/+}$IHCs. The attempt to equalize intracellular buffering of $C a b p 2^{+/+}$and Cabp2 $2^{\text {LacZ/LacZ }}$ IHCs was promising with nearly 
matched sustained exocytosis (Figure 3.13E; $\mathrm{p}>0.05$, Student's t-test). Cabp2 $2^{\text {LacZ/LacZ }}$ IHC still tended to show elevated capacitance increments and reduced $\mathrm{Q}_{C a}$ (Figure 3.13E, $\mathrm{E}_{i}$;), which could be attributed to remaining Cabp2 in $\mathrm{Cabp2} 2^{+/+}$IHCs. Nevertheless, the ruptured patch experiment demonstrated that the effect of increased sustained exocytosis is reversible by manipulating the IHC buffer conditions and washing out mobile Cabp2 aside of other small cytosolic proteins.

\subsubsection{Spiral ganglion neuron responses in $\mathrm{Cabp} 2^{\mathrm{LacZ} / \mathrm{LacZ}}$ animals}

To probe postsynaptic responses to presynaptic vesicle fusion at IHC ribbon synapses Anna Gehrt performed extracellular recordings of spontaneous and evoked spiral ganglion neuron (SGNs) firing activity in 5 to 8-week-old animals. For wild-type data we pooled $\mathrm{Cabp}^{+/+}$littermates and unrelated wild-type mice with $\mathrm{C} 57 \mathrm{~B} 1 / 6 \mathrm{~J}$ background to increase the number of recordings after finding them statistically indistinguishable.

First, spontaneous firing rates of Cabp2 $2^{\mathrm{LacZ} / \mathrm{LacZ}}$ and control fibers were recorded (Figure 3.14A). In $C a b p 2^{L a c Z / L a c Z}$ animals the spontaneous spiking activity of SGNs rendered a significantly higher number of low spontaneous rate fibers compared to wild-type control fibers $\left(\mathrm{Cabp}^{+/+}\right.$ $: \mathrm{n}=160, \operatorname{Cabp}^{\text {LacZ } / \text { LacZ }}: \mathrm{n}=44 ; \mathrm{p}<0.001$, Kolmogorov-Smirnov test). To record the evoked activity of the fibers poststimulus time histograms (PST) of SGNs were obtained by recording the spiking during the presentation of $50 \mathrm{~ms}$ tone burst at the characteristic frequency of the fiber and at a stimulus rate of $5 \mathrm{~Hz}$ (Figure 3.14B). The PSTs are characterized by a high peak or onset rate followed by an adapting period. Spike onset and adapted firing rates were determined by calculating firing rates in $0.5 \mathrm{~ms}$ bins after stimulus onset and at the end (35-45 ms) of the tone burst. In $C a b p 2^{\text {LacZ/LacZ }}$ animals both, onset and steady-state rates were significantly lower than the firing rates of $\mathrm{Cabp}^{+/+}$control fibers (Figure 3.14B; peak rate: $\mathrm{p}<0.001$, Student's t-test; adapted rate: $p<0.05$, Student's t-test). For the $50 \mathrm{~ms}$ PST recordings the stimulus was presented 200 times with an inter-stimulus-interval (ISI) of $50 \mathrm{~ms}$ and the average was calculated. The peak firing rate vs. steady-state rates of SGNs demonstrated a similar reduction in onset and adapted firing rates of Cabp $2^{\text {LacZ/LacZ }}$ SGNs (Figure 3.14C). Moreover, in Cabp $2^{\text {LacZ/LacZ }}$ animals SGNs exhibited higher first spike latencies $\left(\mathrm{Cabp}^{+/+}: 4.9 \pm 0.2 \mathrm{~ms}\right.$; Cabp2 $2^{\mathrm{LaCZ} / \mathrm{LacZ}}: 6.1 \pm 0.3 \mathrm{~ms}$; $\mathrm{p}<0.001$; Wilcoxon rank sum test) and more variable responses of the first spikes meaning more jittered responses $\left(C_{a b p} 2^{+/+}: 6 \pm 1.5 \mathrm{~ms}^{2}, C_{a b p} 2^{\text {LacZ } / L a c Z}: 11.5 \pm 0.36 \mathrm{~ms}^{2} ; \mathrm{p}<0.05\right.$; Figure 3.14D).

To investigate SGN responses to prolonged stimuli, comparable to presynaptic patch-clamp recordings A. Gehrt used $500 \mathrm{~ms}$ tone bursts at $0.5 \mathrm{~Hz}$ and recorded 50 repetitions of PSTs per SGN (Figure 3.14E). Onset rates were unaltered in Cabp $2^{\text {LacZ/LacZ }}$ SGNs and adapted steady-state firing rates tended to be reduced, but this did not reach statistical significance.

In addition to PSTs SGN firing rates in response to sound intensity or rate-level functions were recorded. For these experiments 25 tone burst per sound pressure level were presented sepa- 
A

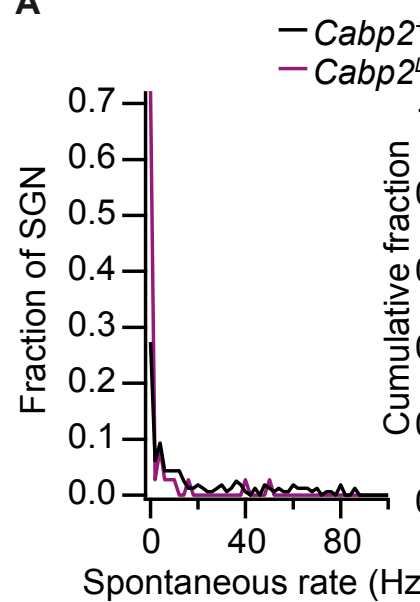

C

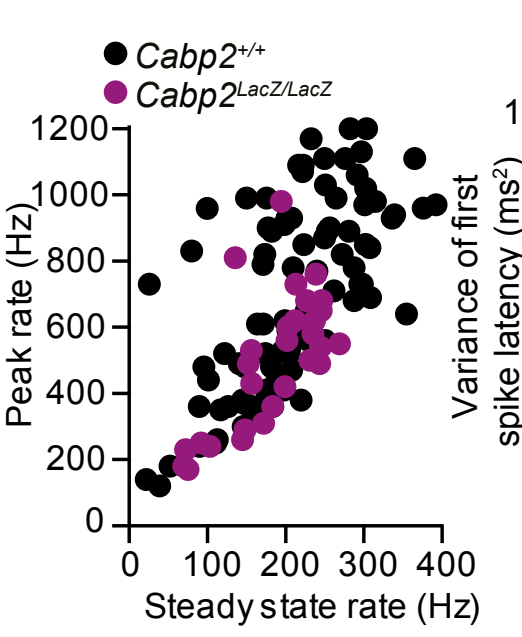

D

pontaneous rate (spikes/s)

B

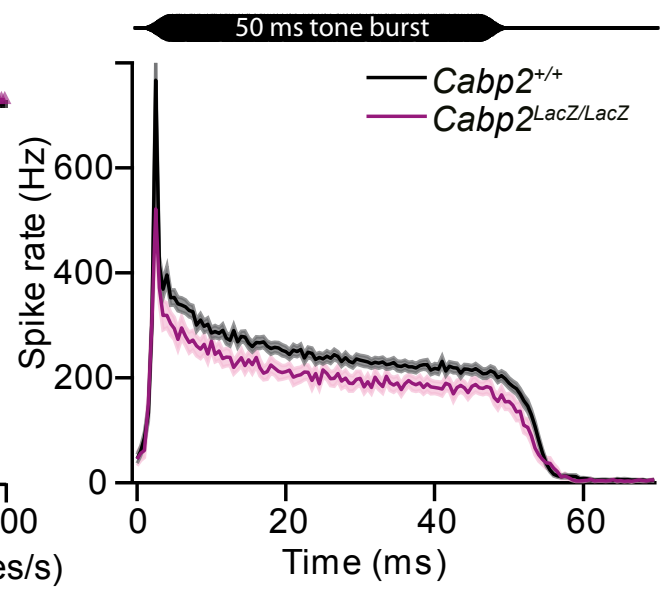

E

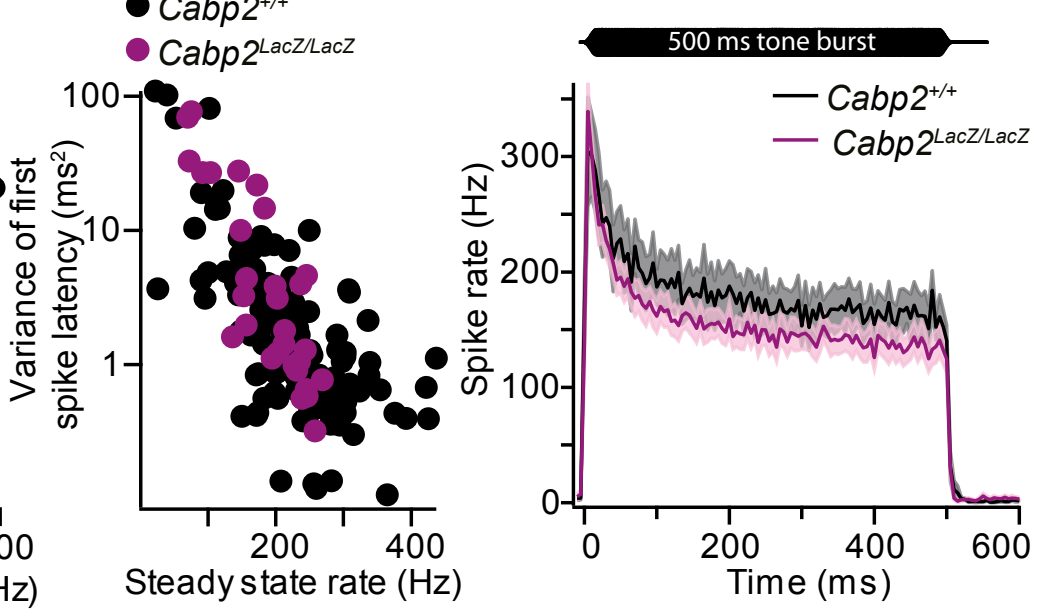

Figure 3.14: SGN responses: (A) Histogramm of spontaneous firing activity of SGNs recorded in $C a b p 2^{L a c Z / L a c Z}$ and $C a b p 2^{+/+}$mice showing a higher fraction of low spontaneous rate fibers in $C a b p 2^{L a c Z / L a c Z}$ SGNs (left). Cumulative plot of spontaneous firing of SGNs (right). (B) PSTs presented as the grand mean of Cabp2 $2^{+/+}(\mathrm{n}=112)$ and $C a b p 2^{\text {LacZ/LacZ }}(\mathrm{n}=30)$ SGNs after peak alignment, illustrating a reduction in onset and adapted firing rates in Cabp2 mutant mice. (C) Quantification of firing rates of individual SGN summarized in A. The maintenance of the linear relation between peak and adapted rate in $\mathrm{Cabp} 2^{\mathrm{LacZ} / \mathrm{LacZ}}$ animals indicates comparable reduction in onset and adapted SGN firing. (D) Variances of first spike latencies as a function of steady-state rate of single SGNs in $\mathrm{Cabp} 2^{+/+}$and $\mathrm{Cabp} 2^{\mathrm{LacZ} / \mathrm{LacZ}}$ animals. Note the reduced number of SGNs with low variability in first spike latencies recorded in mutant mice. (E) Grand average of peak aligned PSTs of Cabp2 $2^{+/+}(\mathrm{n}=11)$ and Cabp $2^{\text {LacZ/LacZ }}(\mathrm{n}=19)$ fibers in response to $500 \mathrm{~ms}$ tone bursts presented $30 \mathrm{~dB}$ above threshold at the characteristic frequency of the individual unit and a stimulus frequency of $0.5 \mathrm{~Hz}$. Onset rates are comparable between $\mathrm{Cabp}^{+/+}$and $\mathrm{Cabp} 2^{\mathrm{LacZ} / \mathrm{LacZ}}$ , but adapted rates tend to be reduced.

rated by $5 \mathrm{~dB}$ increments. The sound intensity ranged between $20 \mathrm{~dB}$ below and $50 \mathrm{~dB}$ above the threshold of the fiber. Per intensity step the spike number within a fixed time window after stimulus 
onset was analyzed (Figure 3.15A). The maximum steepness of the respective rate-level function

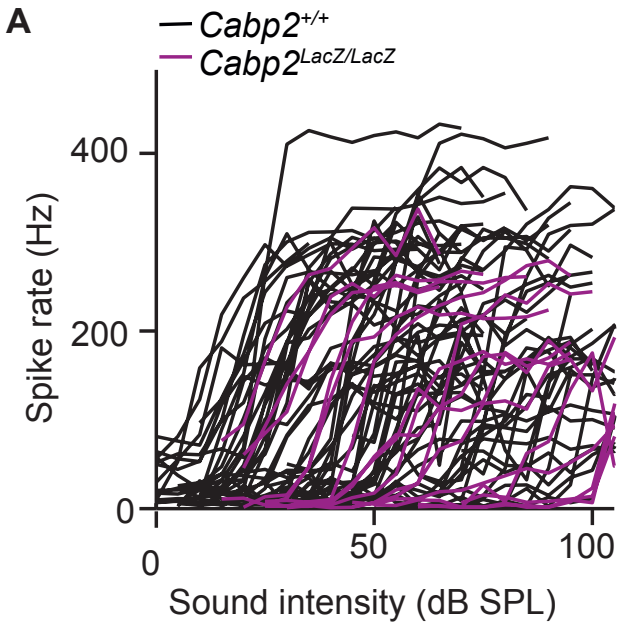

B

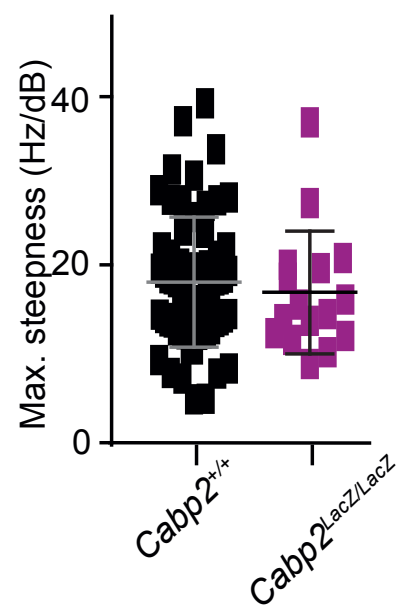

C

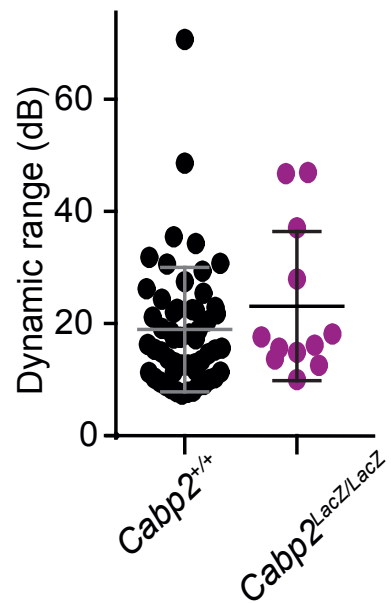

Figure 3.15: Rate-level functions are normal in $C a b p 2^{\mathrm{LacZ} / \mathrm{LacZ}}$ animals: (A) Rate-level function of individual $\mathrm{Cabp}^{+/+}(\mathrm{n}=63)$ and $\mathrm{Cabp} 2^{\mathrm{LacZ} / \mathrm{LacZ}}(\mathrm{n}=16)$ fibers with similar distribution of low and high spike rate fibers in response to sound intensity. (B) Graph showing the maximum steepness of the rate-level functions from A of individual SGNs. Mean \pm s.e.m. overlay as grey/black lines. (C) Dynamic range of individual SGNs, derived from A is not altered in Cabp2 $2^{\text {LacZ/LacZ }}$ SGNs compared to Cabp2 $2^{+/+}$controls.

was determined by fitting linear functions between recordings of consecutive $5 \mathrm{~dB}$ sound intensity steps. The maximum steepness between both genotypes was comparable (Figure 3.15B; $\mathrm{p}>$ 0.05 , Wilcoxon rank sum test). To calculate the dynamic range of a fiber response, the rate-level function was fitted with a sigmoidal function as described in previous work (Taberner and Liberman, 2005).The dynamic range of a SGN is defined as range of sound pressure levels in which the evoked SGN firing rate increases from $10-90 \%$. SGNs of $\mathrm{Cabp} 2^{+/+}$and $\mathrm{Cabp} 2^{\mathrm{LaCZ} / \mathrm{LacZ}}$ mice exhibited comparable dynamic ranges (Figure 3.15C; $p>0.05$, Wilcoxon rank sum test).

The unaffected rate-level functions recorded in $\mathrm{Cabp} 2^{\mathrm{LacZ} / \mathrm{LacZ}}$ animals are inline with the DPOAEs showing intact active amplification by OHCs. Impaired OHC functions could otherwise result in steeper growths of rate-level functions and firing activity spanning a narrower stimulus intensity range.

In summary, SGNs in Cabp $2^{\mathrm{LacZ} / \mathrm{LacZ}}$ animals revealed reduced spontaneous and evoked firing rates. The lower fraction of spontaneous SGN firing might be a consequence of a lower number of spontaneous $\mathrm{Ca}_{V} 1.3$ channel opening at IHC resting membrane potential, while the reduction in evoked firing rates might be caused by $\mathrm{Ca}_{V} 1.3$ inactivation during sound stimulation (at least upon $50 \mathrm{~ms}$ tone bursts at $5 \mathrm{~Hz}$ ). It seems as if $\mathrm{IHC} \mathrm{Ca}_{V} 1.3 \mathrm{Ca}^{2+}$ channels require a $2 \mathrm{~s}$ recovery time to respond to consecutive stimuli due to the comparable onset firing rates between mutant 
and wild-type SGNs recorded at $0.5 \mathrm{~Hz}$. The reduction in adapted firing rates in SGN recordings of $\mathrm{Cabp} 2^{\mathrm{LaCZ} / \mathrm{LacZ}}$ animals supports the idea that increased sustained exocytosis is not detected by the postsynaptic neuron, which otherwise should have increased the adapted firing rates instead. Therefore, I propose that the excess exocytosis observed by $\mathrm{C}_{m}$ recordings reflects extrasynaptic release resulting from impaired $\mathrm{Ca}^{2+}$ buffering.

The reduced precision of SGN firing due to an increase in first spike latencies and higher jitter in addition to the reduced onset and adapted rates could account for the reduction in ABR wave I amplitude observed in $C a b p 2^{L a c Z / L a c Z}$ animals and could therefore explain the hearing impairment observed in DFNB93.

\subsubsection{Gene-therapeutic approach to rescue DFNB93}

Finally, I wanted to explore the feasibility of gene therapy as a potential future treatment for DFNB93. In 2012, a breakthrough in the field of gene therapy of human deafness occurred with the restoration of hearing in mice lacking Vglut3. As mentioned earlier (Introduction 1.5), in these experiments, hearing was restored in Vglut3 knock-out mice two weeks after AAV1-mediated replacement of Vglut3 via postnatal injection (Akil et al., 2012). Dr. Kirsten Reuter identified $\mathrm{AAV} 2 / 1$ with the gene of interest following a human $\beta$-actin promoter as suitable for protein expression in IHCs (Reisinger et al., 2011; Reuter, 2012). Therefore I subcloned mouse Cabp2 transcription variant 1 (GeneBank:NM_013878; long Cabp2 isoform) into an AAV2/1 backbone (AAV-HBA-EWB) for the expression of C-terminally EGFP-tagged Cabp2.

Dr. Sangyong Jung injected the virus into the mouse otocyst of the left ear at embryonic day E11.5-12.5. Successful surgical intervention and the functionality of the virus were tested in wildtype mice of C57B1/6J background. Near normal ABR amplitudes and thresholds were recorded in 3-week-old animals by our technician Nadine Hermann of both, the ipsi- and contralateral ear demonstrating that the surgical procedure and viral gene transfer of a tagged Cabp2 does not interfere with hearing per se (Figure 3.16B-D). Subsequently, I isolated the organ of Corti and fixed the tissue to verify viral transduction by confocal microscopy (Figure 3.16E). IHCs of injected ears showed homogenous Cabp2-EGFP expression with no indication of aggregate formation. From the first 9 wild-type animals an IHC transduction efficiency of $88.8 \%$ was observed, by counting Cabp2-EGFP positive cells in respect to the total number of cells in a defined apical region ( 7-14 $\mathrm{kHz}$ region).

After verifying the functionality of the AAV2/1-Cabp2-EGFP, Sanyong injected the left ear of Cabp $2^{\mathrm{LaCZ} / \mathrm{LaCZ}}$ embryos and ABRs were recorded in both ears to compare the effect of the viral construct on hearing. For these experiments, 5-week-old animals were used, given the greater hearing impairment in the older mice (Figure $3.6 \mathrm{~A}_{i}$ ). Here, the contralateral ear was recorded first, plugged thereafter with a subsequent recording from the ipsilateral ear. ABR recordings of the first 
A

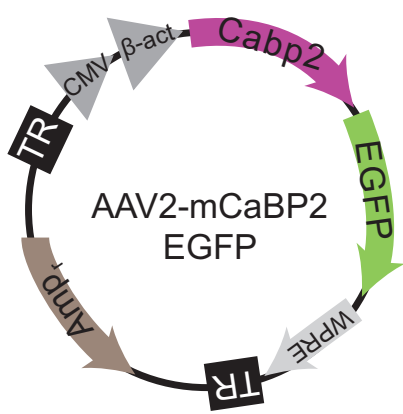

D

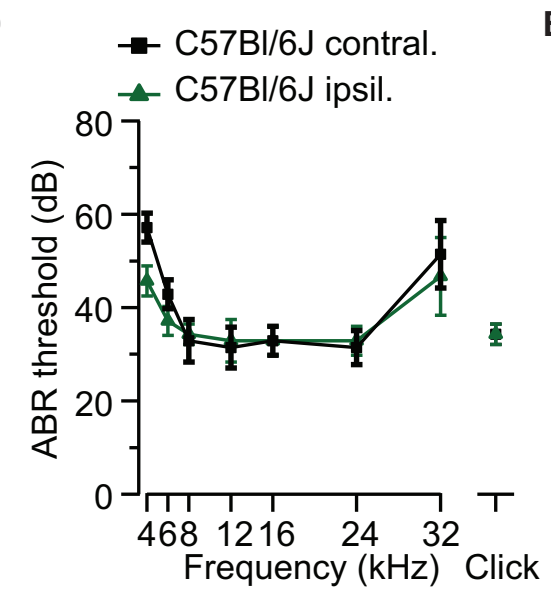

B $\quad$ - C57BI/6J contral. - C57BI/6J ipsil.

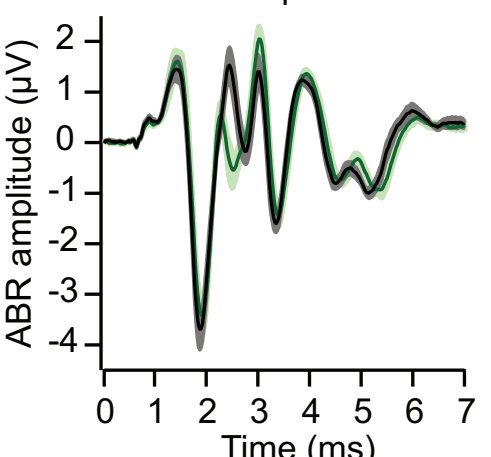

E

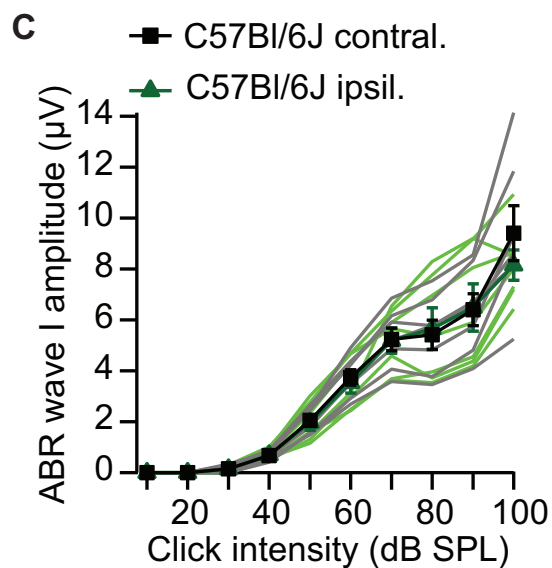

Click intensity (dB SPL)

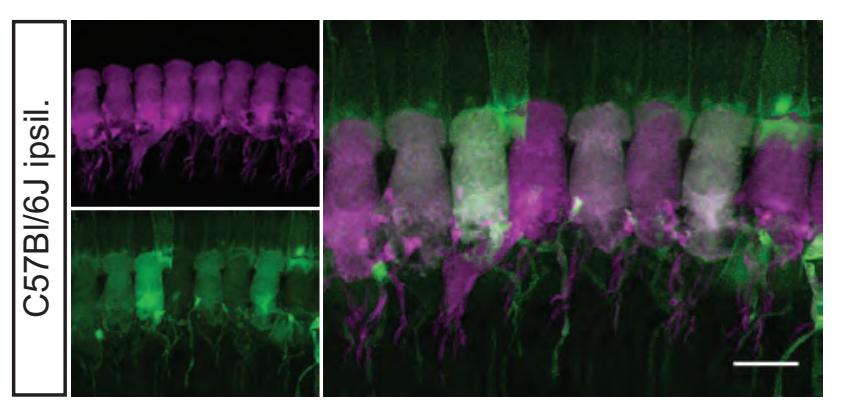

Figure 3.16: AAV2/1-Cabp2-pEGFP transduces IHCs without affecting hearing in wild-type animals: (A) Schematic drawing of the viral construct highlighting CMV-enhancer and $\beta$-actin promoter followed by the EGFP-tagged Cabp2 sequence. (B) Comparable grand average ABR amplitudes of ipsilateral (injected) and contralateral (non-injected) ears recorded in C57B1/6J animals $(\mathrm{n}=7)$. (C) ABR wave I amplitudes of all recordings obtained from B. (D) ABR thresholds of same animals as in B are normal. (E) Immunostaining of AAV2/1-Cabp2-EGFP infected IHC in green and IHC-specific calretinin staining in magenta showing high transduction rate of the viral construct. Scale bar represents $10 \mu \mathrm{m}$.

10 animals showed no sign of improved hearing performance. ABR amplitudes were not affected by the treatment. ABR wave I tended to show shorter latencies (Figure 3.17A), ABR-thresholds were comparable between transduced and untreated ears of $C a b p 2^{L a c Z / L a c Z}$ animals with significantly lowered hearing threshold by $10 \mathrm{~dB}$ at a frequency of $6 \mathrm{kHz}(\mathrm{p}=0.026$, Student's t-test; Figure 3.17C).

Moreover, $\mathrm{Ca}_{V} 1.3$ inactivation was tested in AAV2/1-Cabp2-EGFP infected IHC of p15-21 animals by recording $500 \mathrm{~ms}$ inactivating currents (Figure 3.17D). $\mathrm{Ca}^{2+}$ currents during $500 \mathrm{~ms}$ depolarizations were unchanged after treatment with comparable $\mathrm{R}_{500}$ values between injected 
A

- Cabp2 2acZlacz contral.

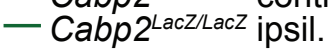

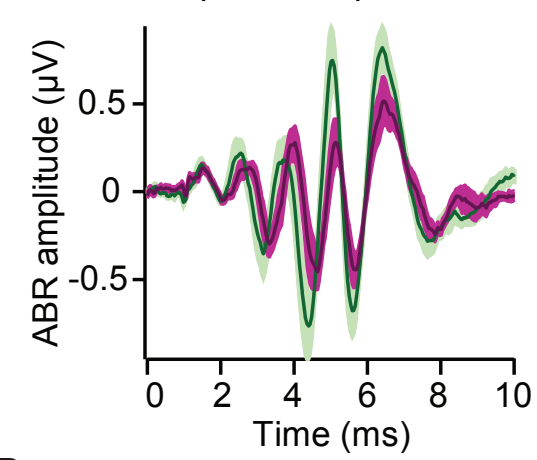

D

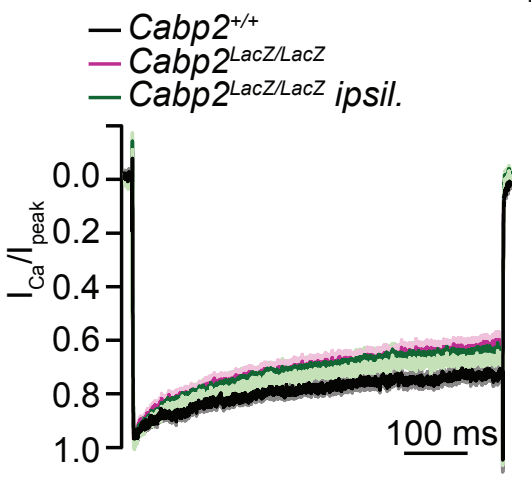

B

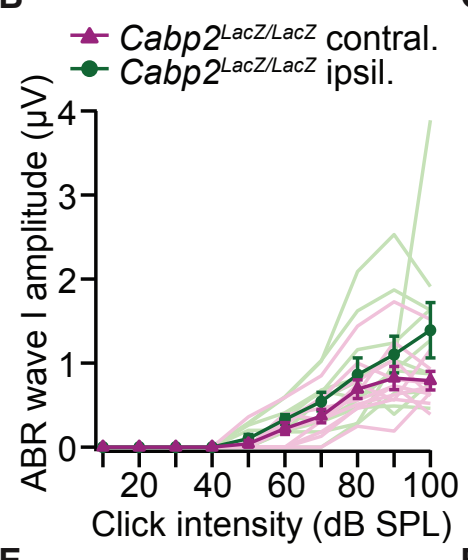

E

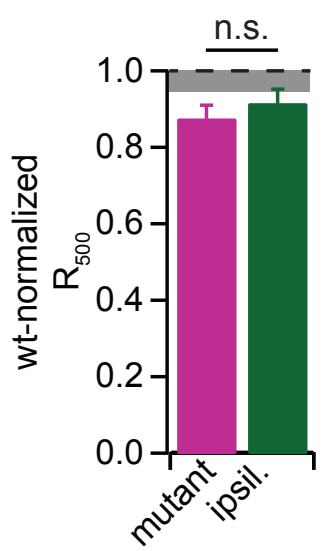

C

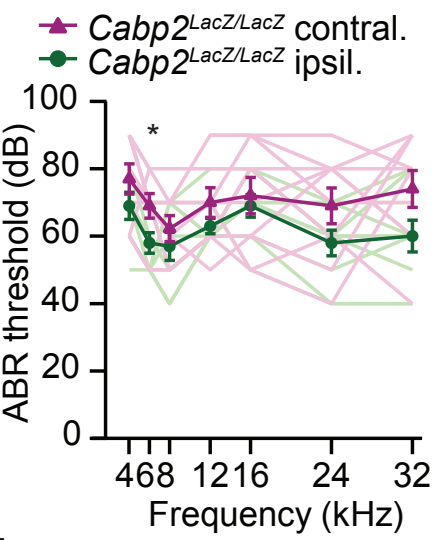

$F$

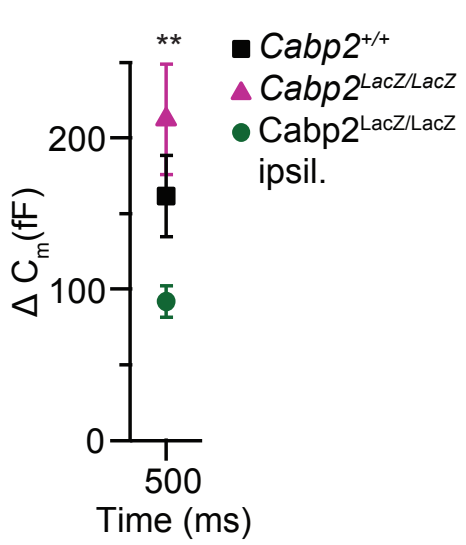

Figure 3.17: Hearing in $C a b p 2^{\mathrm{LacZ} / \mathrm{LacZ}}$ animals is not improved by the injection of AAV2/1Cabp2-EGFP: (A) Grand average of ABR amplitudes from ipsilateral and contralateral ears of Cabp2 $2^{\text {LacZ } / \text { LacZ }}$ animals $(\mathrm{n}=10)$. (B) ABR wave I amplitudes with overlay of the grand average derived from A. Wave I amplitude after AAV2/1-Cabp2-EGFP injection are comparable before and after treatment. (C) ABR thresholds recorded in both ears with comparable hearing thresholds. At a frequency of $6 \mathrm{kHz}$ thresholds are significantly lowered after virus injection. (D) Normalized average $\mathrm{I}_{C a}$ in response to $500 \mathrm{~ms}$ depolarizations of ipsilateral $(\mathrm{n}=10), \operatorname{Cabp} 2^{\text {LacZ/LacZ }}(\mathrm{n}=16)$ and $\mathrm{Cabp2}^{+/+}(\mathrm{n}=12)$ IHCs. (E) For clarity the $\mathrm{R}_{500}$ values are normalized to Cabp2 $2^{+/+} \mathrm{R}_{500}$ $(\mathrm{n}=12)$ and the shaded gray area represents the s.e.m. of $\mathrm{Cabp2}^{+/+} \mathrm{R}_{500}$. Note that $\mathrm{R}_{500}$ is comparable between ipsilateral and Cabp $2^{\text {LacZ/LacZ }}$ IHCs. (F) Respective average of $\Delta \mathrm{C}_{m}$ after $500 \mathrm{~ms}$ depolarizations. Sustained exocytosis is significantly lowered in Cabp2 overexpressing IHC compared to Cabp2 $2^{\text {LacZ/LacZ }}$ IHC ( $p=0.005$, Wilcoxon rank sum test).

and untreated Cabp2 $2^{\text {LacZ/LacZ }}$ IHCs (Figure 3.17D, E). However, IHCs capacitance traces show a significant reduction in sustained exocytosis in response to $500 \mathrm{~ms}$ depolarizations in infected Cabp2 overexpressing IHCs compared to Cabp2 $2^{\mathrm{LaCZ} / \mathrm{LaCZ}}$ and Cabp2 $2^{+/+}$IHCs (Figure 3.17F; $\mathrm{p}=$ 0.006, Wilcoxon rank sum test), supporting the idea that Cabp2 buffers intracellular $\mathrm{Ca}^{2+}$ which is not involved in hearing impairment observed in $\mathrm{Cabp} 2^{\mathrm{LacZ} / \mathrm{LacZ}}$ mice. 
In summary, viral rescue experiments of $C a b p 2^{\text {LacZ/LacZ }}$ animals so far demonstrate that moderate hearing impairment induced by Cabp2 lacking C-terminal EF-hands cannot be rescued by mere overexpression of Cabp2-EGFP. $\mathrm{I}_{C a}$ inactivation is not altered by introducing Cabp2-EGFP but the expression and $\mathrm{Ca}^{2+}$ binding of $\mathrm{Cabp} 2$ can be assumed since sustained exocytosis is decreased in $\mathrm{Cabp} 2^{\mathrm{LacZ} / \mathrm{LacZ}}$ IHCs. While sustained exocytosis is significantly lower in transduced IHC compared to Cabp $2^{\mathrm{LaCZ} / \mathrm{LaCZ}}$, hearing is only mildly affected, which confirms that the proposed reduction in $\mathrm{Ca}^{2+}$ buffering in the absence of Cabp2 does not affect hearing. 


\section{Discussion}

$\mathrm{Ca}_{V}$ 1.3-mediated $\mathrm{Ca}^{2+}$ influx triggers sound induced glutamate release at ribbon synapses of IHCs. To enable faithful sound encoding, $\mathrm{Ca}_{V} 1.3$ channels found in IHCs exhibit weak inactivation properties (Cui et al., 2007; Grant and Fuchs, 2008; Johnson and Marcotti, 2008; Yang et al., 2006), unlike $\mathrm{Ca}_{V} 1.3$ expressed in heterologous expression systems (Yang et al., 2006). However, to date, the regulatory elements preventing $\mathrm{Ca}^{2+}$ channel inactivation in IHCs remain unknown. CaBPs, EF-hand $\mathrm{Ca}^{2+}$ binding proteins, are good candidates for inhibiting $\mathrm{Ca}_{V} 1.3$ inactivation in IHCs and were shown to antagonize CaM-induced CDI of multiple VGCC isoforms (Cui et al., 2007; Yang et al., 2006; Zhou et al., 2004). Among CaBPs, so far only regulation of $\mathrm{IHC} \mathrm{Ca}_{V} 1.3$ by CaBP4 was tested using knock-out mice (Cui et al., 2007). These mice exhibited normal ABR thresholds and amplitudes and a very subtle increase in $\mathrm{Ca}_{V} 1.3 \mathrm{CDI}$. This called into question a substantial modulation of $\mathrm{Ca}^{2+}$ channels inactivation in IHCs by CaBP4, which however seems to assume an important role in retinal photoreceptors (Haeseleer et al., 2004). The discovery of a missense mutation in the human $C A B P 2$ gene, resulting in moderate-to-severe hearing impairment (DFNB93; (Tabatabaiefar et al., 2011)), makes CaBP2 the most promising candidate regulator of IHC Ca $_{V} 1.3$ inactivation (Schrauwen et al., 2012) among CaBPs. In the present study, I combined mouse genetics, electrophysiology, immunohistochemistry and molecular assays to decipher the role of $\mathrm{CaBP} 2$ in the cochlea and to identify the disease mechanism of DFNB93. I demonstrated that $\mathrm{CaBP} 2$ (i) inhibits $\mathrm{Ca}_{V} 1.3$ inactivation, (ii) deficient mice have a progressive hearing impairment, which likely results from increased steady-state inactivation of $\mathrm{IHC} \mathrm{Ca}_{V} 1.3 \mathrm{Ca}^{2+}$ channels.

\section{1 $\operatorname{Cabp2} 2^{L a c Z / L a c Z}$ mice are a suitable animal model for DFNB93}

The Cabp2 $2^{\mathrm{LacZ} / \mathrm{LacZ}}$ mouse mutant was used to investigate whether Cabp2 is required for hearing and synaptic transmission in IHCs and to elucidate the mechanism of DFNB93-induced hearing impairment. Hearing was tested in these animals at the system's level by measuring (i) DPOAEs to reveal potential cochlear amplification deficits and (ii) ABRs to assess sound encoding (Figure 3.6). Cabp $2^{\text {LacZ/LacZ }}$ mice showed elevated ABR thresholds, while cochlear amplification remained intact. ABR wave I amplitudes, reflecting synchronous firing activity of SGNs, were reduced and delayed. The combination of a reduced ABR wave I amplitude and intact DPOAEs points towards impaired sound encoding at the IHC ribbon synapse ((Moser et al., 2013); Section 1.4.3).

The $C a b p 2^{\text {LacZ/LacZ }}$ mouse model resembles affected human patients in many aspects. In both, Cabp $2^{\text {LacZ/LacZ }}$ mice and DFNB93 patients hearing impairment was symmetric (bilateral). Moreover, the increase in hearing threshold was more pronounced in the mid-frequency regions, being moderate in 8-week-old (mature) Cabp2 $2^{\mathrm{LacZ} / \mathrm{LacZ}}$ animals (threshold elevation: $30 \mathrm{~dB}$; Figure 3.6) and moderate-to-severe (threshold elevation: 50-90 dB) in adult human patients (Schrauwen et al., 
2012). DFNB93 was classified as autosomal recessive non-syndromic hearing impairment. This is comparable to the animal model with a recessive hearing deficit, a normal lifespan and no obvious physical or behavioral abnormalities.

Nevertheless, two observations differed between human DFNB93 patients and Cabp2 $2^{\text {LacZ/LacZ }}$ animals. Firstly, DFNB93 patients displayed impaired cochlear amplification, which was absent in the mutant mouse strain. A similar observation was made for a human loss-of-function mutation OTOF, affecting the gene encoding for otoferlin (DFNB9; (Rodriguez-Ballesteros et al,, 2003)). DFNB9 patients exhibited progressively deteriorating cochlear amplification after the first two years, while in otoferlin knock-out mice cochlear amplification remained intact (Roux et al., 2006). In this regard, it is important to consider that lab animals are raised in a protected environment. Human patients on the other hand can be exposed to environmental factors during their lifetime that may contribute to amplification deficits. Moreover, it remains to be tested whether cochlear amplification might deteriorate in $\mathrm{Cabp} 2^{\mathrm{LacZ} / \mathrm{LacZ}}$ at higher ages.

Secondly, the course of hearing impairment was different in human patients as compared to Cabp $2^{\text {LacZ/LacZ }}$ animals. Humans exhibited a history of prelingual moderate-to-severe hearing impairment (Tabatabaiefar et al., 2011), without any indication of progressive hearing loss as I observed in $C a b p 2^{\mathrm{LacZ} / \mathrm{LacZ}}$ mice. However, it is difficult to determine if hearing impairment in humans is progressive, as human hearing starts around the $20^{\text {th }}$ gestational week, long before hearing can be tested after birth. On the contrary, in mice hearing onset starts at postnatal day 14-15, one week before ABRs were recorded. Due to limitations in examining patients on a regular basis during early life, it is well justified to use the animal model instead to investigate the course of DFNB93.

To conclude, $\operatorname{Cabp} 2^{\mathrm{LacZ} / \mathrm{LacZ}}$ mice present an auditory synaptopathy and are proposed to represent a valuable model for detailed investigation of the disease mechanism of DFNB93.

\subsection{Cabp2 expression in $C a b p 2^{L a c Z / L a c Z}$ animals}

In the present study, Cabp2 expression was assessed in $C a b p 2^{L a c Z / L a c Z}$ mice by $\beta$-galactosidase staining of the cochlea and the retina. Cochlear inner, outer, and vestibular hair cells, and some retinal neurons were Cabp2-positive. Similar to my observation, a cytosolic Cabp2 expression was reported (i) in IHCs by immunostainings (Cui et al., 2007), which however lacked the negative control by knock-out tissue, and (ii) in the retina by mRNA profiling approaches using northern blot analysis (Haeseleer et al., 2000). Preliminary immunostainings of retinal slices suggest Cabp2 fluorescence in horizontal cells (Figure 3.5). So far, no indication of Cabp2 expression in the brain has been reported. Samples from cortex and cerebellum were negative for Cabp2 mRNA (Haeseleer et al., 2000) and screenings for mRNA expression in other brain tissues, in- 
cluding the thalamus, hippocampus, amygdala, etc. suggested the absence of Cabp2 (Schrauwen, unpublished).

\subsection{Experiments in heterologous expression systems}

\subsubsection{CABP2-wt increases the voltage-sensitivity and inhibits CDI of $\mathrm{Ca}_{V} 1.3$ channels}

Heterologous expression systems are extensively used to investigate channel modulations by interactions with other proteins (Gebhart et al., 2010; Gregory et al., 2013), disease causing mutations (Baig et al., 2011; Hans et al., 1999; Pinggera et al., 2015) and channel modifications due to alternative splicing (Huang et al., 2013; Koschak et al., 2001; Tan et al., 2011). Also our first analysis of $\mathrm{Ca}_{V} 1.3$ modulation by $\mathrm{CABP} 2$ has been performed using heterologous expression in HEK293/T cells (Schrauwen et al., 2012). However, for continued investigations I employed, to my knowledge, for the first time HEK293 cells stably expressing the $\mathrm{Ca}^{2+}$ activated small conductance $\mathrm{K}^{+}$channel SK3-1 for studying the biophysical properties of $\mathrm{Ca}^{2+}$ channels. I chose this approach to avoid cytotoxicity associated with non-inactivating $\mathrm{Ca}^{2+}$ influx into HEK293 cells, reasoning that $\mathrm{Ca}^{2+}$ induced SK3-mediated hyperpolarization would provide negative feedback to $\mathrm{Ca}^{2+}$ influx. Indeed, compared to HEK293/T cells I found much more viable transduced cells. Moreover, in addition to the analysis of CABP2-EF ${ }^{\Delta 3 / 4} \mathrm{I}$ also studied the effect of a sequence variant found by the van Camp lab that was predicted to interfere with $\mathrm{Ca}^{2+}$ binding by $\mathrm{EF} 1$. The functions of CABP2-wt reported by our previous study were (i) decreased $\mathrm{Ca}^{2+}$ current density amplitudes (i.e. $\mathrm{Ca}^{2+}$ currents normalized to the cell capacitance) and (ii) inhibition of $\mathrm{Ca}_{V} 1.3_{42}$ induced CDI. Both effects were less pronounced in the presence of the CABP2-EF ${ }^{\Delta 3 / 4}$ (DFNB93).

Using HEK293/hSK3-1 cells I confirmed that CABP2-wt completely abolishes CDI of heterologously expressed $\mathrm{Ca}_{V} 1.3_{42}$ (Figure 3.1). However, I could not reproduce the previously observed reduction of $\mathrm{Ca}^{2+}$ current density by CABP2-wt. Most likely this points towards the expected cytotoxicity of non-inactivating $\mathrm{Ca}^{2+}$ influx in our previous study that probably led to a bias for cells with low $\mathrm{Ca}^{2+}$ channel expression. Indeed, a decrease in current amplitude upon elevation of $\mathrm{CaBP}$ expression was shown for CaBP4 in a recent study (Yang et al., 2014) and is a general challenge for $\mathrm{Ca}^{2+}$ channel modulation studies in heterologous expression system (Cui et al., 2007; Yang et al., 2006, 2014; Zhou et al., 2004). Therefore, a reduction of cytotoxicity, e.g. by a negative feedback to $\mathrm{Ca}^{2+}$ influx by SK channel-mediated hyperpolarization employed here, seems important for studies in heterologous expression systems to more closely model $\mathrm{CaBP}$ and perhaps more generally $\mathrm{Ca}_{V}$ function in native cells. However, one disadvantage of HEK293/hSK3-1 cells is the short experimental time window of approximately 2-3 weeks owing to the fact, that HEK293/hSK3-1 cells need to be young to stably express the hSK3-1 channel and four additional DNA-plasmids (CABP2, $\mathrm{Ca}_{V} \beta, \mathrm{Ca}_{V} \alpha 1, \mathrm{Ca}_{V} \alpha_{2} \delta$ ). Figure 3.3 shows low current densities in cells coexpressing $\mathrm{Ca}_{V} 1.3_{42-A 2123 V}$ and $\mathrm{CABP} 2-\mathrm{EF}^{\Delta 3 / 4}$ recorded after the high expression period. 
Nevertheless, I found the limited experimental time period is an acceptable trade off, can be bypassed with proper planning and ultimately improves the success rate of recordings of low signal to noise ratios resulting in a higher quality of the measurements.

My studies of the modulation of $\mathrm{Ca}_{V} 1.3 \mathrm{Ca}^{2+}$ channels by CABP2-wt or $-\mathrm{EF}^{\Delta 3 / 4}$ in HEK cells indicated two candidate mechanisms for DFNB93. Firstly, CABP2 lacking EF-hand 3 and 4 might induce hearing impairment due to decreased voltage-sensitivity shifting IHC transmitter release out of the range of the physiological receptor potential. Secondly, DFNB93 might be a consequence of an enhanced steady-state inactivation of a $\mathrm{Ca}_{V} 1.3$ channels, since $\mathrm{Ca}_{V} 1.3$ channels are already activated at IHC resting potential ((Zampini et al., 2010), see below). CABP2-EF ${ }^{\Delta 3 / 4}$ only partially suppressed CDI and had no effect on voltage-sensitivity. Regulation of VGCC activation was also reported for $\mathrm{CaBP} 4$ which shifts the activation of $\mathrm{Ca}_{V} 1.4 \mathrm{Ca}^{2+}$ channels to more hyperpolarized potentials in HEK293/T cells (Haeseleer et al., 2004). The shifted activation range of $\mathrm{Ca}_{V} 1.4$ was suggested by Haeseleer and colleagues to account for the reduced light sensitivity of photoreceptor synapses in $\mathrm{Cabp} 4^{-/}$mice and was proposed as underlying cause for incomplete congenital stationary night blindness 2 (CSNB2; (Zeitz et al., 2006)). However, when ultimately testing $\mathrm{Ca}_{V} 1.3$ voltage-sensitivity in IHCs, I did not observe the expected shift in $\mathrm{Ca}_{V} 1.3$ activation range ruling out hypothesis 1 as underlying cause of DFNB93. The discrepancy between HEK293 and IHC recordings suggests a compensation of $\mathrm{Ca}_{V} 1.3$ voltage-sensitivity by other proteins expressed in IHCs. Potential candidates are other CaBPs (CaBP1,CaBP4, CaBP5 (Cui et al., 2007; Yang et al., 2006)), or PDZ domain containing proteins such as harmonin (Gregory et al., 2013) or GAIP-interacting protein C-terminus (GIPC3, Ohn et al, unpublished). These PDZ domain containing proteins were both shown to regulate $\mathrm{Ca}_{V} 1.3$ activation in IHCs and induce a $\mathrm{V}_{\text {half }}$ shift when being knocked out (Gregory et al., 2013). However, similar differences between HEK293 and inner hair cell recordings were also shown for RIM proteins, which regulate $\mathrm{Ca}_{V} 1.3$ in HEK293/T cells (Gebhart et al., 2010) but show no $\mathrm{Ca}_{V} 1.3$ regulation phenotype in RIM2 deficient IHCs, meaning that $\mathrm{V}_{\text {half }}$ and $\mathrm{k}_{\text {act }}$ are normal in these mice (Jung et al., 2015b). Given the complexity of the molecular machinery found in inner hair compared to HEK293 cells, it is justified that $\mathrm{Ca}_{V} 1.3$ modulation experiments in heterologous expression system can only partially reconstitute IHC conditions.

\subsection{IHC $\mathrm{Ca}^{2+}$ channels display increased inactivation}

\subsection{1 $\mathrm{Ca}^{2+}$-dependent inactivation is not affected in $\mathrm{Cabp}^{\mathrm{LacZ} / L a c Z}$}

Perforated-patch recordings of $\mathrm{IHC} \mathrm{Ca}{ }^{2+}$ currents rendered hypothesis 1, DFNB93 arises from decreased voltage-sensitivity of $\mathrm{Ca}_{V} 1.3$ channels in the absence of functional Cabp2 unlikely. Moreover, $\mathrm{Ca}_{V} 1.3$ channels in IHCs of young Cabp2 $2^{\mathrm{LaCZ} / \mathrm{LacZ}}$ animals exhibited only a trend towards 
IHC Ca ${ }^{2+}$ channels display increased inactivation

increased CDI (Figure 3.10), suggesting that other factors might antagonize CaM and thereby inhibit CDI in the absence of Cabp2. In addition to CaBP2, CaBP1, CaBP4, and CaBP5 were also shown to be expressed in IHCs by immunostainings (Cui et al., 2007), however, the localization of $\mathrm{CaBP} 1$ in IHCs remains controversial, since Yang and coworkers proposed a predominant expression of CaBP1 in SGNs (Yang et al., 2014). So far, the interaction of CaBPs with $\mathrm{Ca}_{V} 1.3$ was predominantly investigated in heterologous expression system where CaBP1 (Cui et al., 2007; Findeisen et al., 2013) and CaBP4 (Cui et al., 2007; Yang et al., 2014) were both shown to most potently antagonize $\mathrm{CaM}$-induced $\mathrm{CDI}$, suggesting that they might alternatively inhibit CDI in IHCs, compensating for the absence of CaBP2.

C-terminal autoregulation of long $\mathrm{Ca}_{V} 1.3$ splice variants might be another candidate mechanism that could contribute to the inhibition CDI of at least a fraction of IHC $\mathrm{Ca}_{V} 1.3$ channels. Multiple $\mathrm{Ca}_{V} 1.3$ splice variants have been reported (Bock et al., 2011; Huang et al., 2013; Koschak et al., 2001; Singh et al., 2008). They were described to differ in length of the C-terminal tail of the $\mathrm{Ca}_{V} 1.3$ channel, that contains the $\mathrm{C}$-terminal automodulatory domain, giving rise to short and long variants. Long and short $\mathrm{Ca}_{V} \alpha 1$ splice variants were shown to differentially regulate voltage-dependence of activation and inactivation of the $\mathrm{Ca}^{2+}$ channel upon heterologous expression (Bock et al., 2011; Tan et al., 2011). Recently, the functional role of an alternatively spliced $\mathrm{Ca}_{V} \alpha 1$ subunit was investigated in IHCs of mutant mice. In this study, the long $\mathrm{Ca}_{V} \alpha 1$ variant was mutated within the C-terminal automodulatory domain by adding an HA-tag sequence, resulting in a $\mathrm{Ca}_{V} 1.3$ channel that according to HEK293/T cell experiments behaves in terms of activation and inactivation like the short splice variant (Scharinger et al., 2015; Singh et al., 2008). By using immunostainings with an anti-HA antibody, the long $\mathrm{Ca}_{V} \alpha 1$ variant was proven to be expressed at all ribbon synapses of IHCs (Scharinger et al., 2015) and is characterized by enhanced fast $\mathrm{Ca}_{V} 1.3$ channel inactivation in IHCs (Bock et al., 2011; Singh et al., 2008). The abundance of this self-regulatory long $\mathrm{Ca}_{V} 1.3$ variant at all ribbon synapses of IHCs is consistent with the finding of minor enhancement of CDI upon deletion of Cabp2.

\subsubsection{Increased voltage-dependent inactivation in $\operatorname{Cabp2}^{\mathrm{Lac} Z / L a c Z}$ animals}

Surprisingly, the most striking observation of IHC physiology was an enhanced VDI in the absence of Cabp2. This adds to the above described discrepancy in CDI regulation between native and heterologously expressed $\mathrm{Ca}_{V} 1.3$ as I did not detect a change in VDI upon transient coexpression of CABP2-wt and $\mathrm{Ca}_{V} 1.3$ in HEK293/h-SK3-1 cells. I can offer two not necessarily exclusive explanations for my observation. First, I used the same constructs as the previous study expressing an EGFP-tag at the N-lobe region of CABP2, which might interfere with the regulation of VDI by CABP2-wt. This hypothesis is built on previous work proposing distinct functions of the Nand $\mathrm{C}$-terminal lobe regions of $\mathrm{CaBPs}$, where the $\mathrm{N}$-lobe was proposed to modulate $\mathrm{Ca}^{2+}$ channel 
functions ((Findeisen and Minor, 2010), see below). Future experiments in HEK293/hSK3-1 using untagged $\mathrm{CaBP} 2$ need to address this possibility. Second, and likely more importantly, while I tried to closely mimic the native $\mathrm{Ca}_{V} 1.3$ composition found in IHCs by heterologous expression, remaining differences in molecular composition could explain the discrepancy. I used the $\mathrm{Ca}_{V} \beta_{2 a}$ subunit for my transient expression experiment to compare my recordings to previously published experiments (Schrauwen et al., 2012). The $\mathrm{Ca}_{V} 1.3 \beta_{2 a}$ subunit is used to resemble the predominant expression of $\mathrm{Ca}_{V} 1.3 \beta_{2}$ in IHCs (Neef et al., 2009), whereby the presence of $\mathrm{Ca}_{V} \beta_{2 a}$ remained to be demonstrated. Alongside $\mathrm{Ca}_{V} 1.3 \beta_{2}$, expression of other $\mathrm{Ca}_{V} 1.3 \beta$ subunits in IHCs, although small, was shown either on an mRNA level $\left(\mathrm{Ca}_{V} 1.3 \beta_{1}, \mathrm{Ca}_{V} 1.3 \beta 3\right.$; (Neef et al., 2009)) or by a $\mathrm{Ca}_{V} 1.3 \beta_{4}$ mutant mouse model exhibiting reduces current amplitudes (Kuhn et al., 2009). $\mathrm{Ca}_{V} \beta_{2 a}$ is post-translationally palmitoylated, a modification that was shown to stabilize the $\mathrm{Ca}_{V} 1.3 \beta$-AID complex at the plasma membrane (Restituito et al., 2000), preventing the hinged-lid mechanism during VDI ((Stotz and Zamponi, 2001; Stotz et al., 2000; Tadross et al., 2010); Section 1.3.6.1). Therefore, in case that IHCs express $\mathrm{Ca}_{V} \beta_{2 b}$ instead of $\mathrm{Ca}_{V} \beta_{2 a}$ my experiments on CaBP2 modulation of heterologously expressed $\mathrm{Ca}_{V} 1.3$ channels might have missed this function of CaBP2. Future experiments, designed to reveal (i) the relative abundance of the $\mathrm{Ca}_{V} \beta_{2 b}$-subunits instead of $\mathrm{Ca}_{V} \beta_{2 a}$ at $\mathrm{IHC} \mathrm{Ca}_{V} 1.3$ channels and (ii) the regulation of heterologously expressed $\mathrm{Ca}_{V} 1.3$ channels by $\mathrm{CaBP} 2$ in the presence of $\mathrm{Ca}_{V} \beta_{2 b}$ or other $\mathrm{Ca}_{V} \beta_{S}$ expressed in IHCs will help to clarify this issue. If this hypothesis is correct, it would also demonstrate the specificity of VDI regulation by a specific $\mathrm{CaBP}$ of a particular $\mathrm{Ca}_{V} 1.3$ channel complex. Consistent with this concept a previous report implicated $\mathrm{CaBP} 1$, but not $\mathrm{CaBP} 4$, in mediating VDI upon heterologous $\mathrm{Ca}_{V} 1.3$ expression in the same set of experiments (Cui et al., 2007).

The exact mechanism of VDI inhibition by CaBPs is not known. In biophysical experiments comparing $\mathrm{CaBP} 1$ and $\mathrm{CaM}$ binding to the $\mathrm{Ca}_{V} 1.2$ channel separate functions of $\mathrm{C}$ - and N-lobe regions of CaBP1 were postulated (Findeisen and Minor, 2010). Findeisen and colleagues proposed that $\mathrm{Ca}_{V} 1.2$ channel modulation is accomplished via the $\mathrm{N}$-lobe, while the $\mathrm{C}$-lobe is promoting competition with CaM over the IQ-domain of the $\mathrm{Ca}^{2+}$ channel. So far, both $\mathrm{CaBP} 1$ (Cui et al., 2007) and CaBP2 (by the present study), were implicated in inhibiting VDI and are the only CaBPs that are N-terminally myristoylated (Haeseleer et al., 2000). This modification was suggested to promote $\mathrm{CaBP}$ trafficking and enhance stabilization of the protein at the plasma membrane (Haeseleer et al., 2000). Therefore it is tempting to assume that CaBP2 mediated inhibition of VDI might be due to a direct interaction of the N-lobe with the channel occluding 'lid' complex, that is comprised of the $\mathrm{Ca}_{V} 1.3 \beta$-AID domain ((Dafi et al., 2004; Stotz and Zamponi, 2001; Stotz et al., 2000); Section 1.3.6.1) resulting in the stabilization of the 'lid' in proximity of the plasma membrane. Alternatively, the N-lobe might just indirectly shield the channel pore and prevent interaction between the $\mathrm{Ca}_{V} 1.3 \beta$ subunit and the pore region. However, the modulation of inactivation by the $\mathrm{N}$-terminal myristoylation was shown to be dependent on $\mathrm{Ca}^{2+}$ channel types. 
While $\mathrm{CaBP} 1$ requires the $\mathrm{N}$-terminal myristoylation to positively shift voltage-dependence of activation, accelerate inactivation and prevent $\mathrm{Ca}^{2+}$ dependent facilitation of $\mathrm{Ca}_{V} 2.1$ channels (Few et al., 2005), it is not required for the inhibition of CDI of $\mathrm{Ca}_{V} 2.1$ channels (Findeisen and Minor, 2010). Therefore, the above mentioned hypothesis, the interaction and the shielding need to be tested by determining a direct interaction between $\mathrm{CaBP} 2$ and either the $\mathrm{Ca}_{V} 1.3 \beta$ subunit or the III linker region (AID-domain) using e.g. biophysical experiments such as pull down assays and/or co-immunoprecipitation. To further investigate whether the $\mathrm{N}$-terminal myristoylation is required for the regulation of VDI a CaBP2 mutant form, lacking this modification is required and can be tested by electrophysiological recordings in HEK293 cells.

Alternatively, unaffected CDI in Cabp2 mutants might be a consequence of $\mathrm{Ba}^{2+}$ dependent inactivation, which was shown to mimic CDI or the fast inactivation, but less effectively (Ferreira et al., 1997; Grandi et al., 2010). To prevent fast $\mathrm{Ca}^{2+}$ channel inactivation, divalent ion free recoding conditions are required. To date, $\mathrm{Na}^{+}$permeability of $\mathrm{L}$-type $\mathrm{Ca}^{2+}$ channels was only recorded in ventricular myocytes and tsA201 cells (Brunet et al., 2009; Ferreira et al., 1997) and would be a challenging experiment in IHCs.

\subsubsection{Steady-state inactivation of $\mathrm{Ca}_{V} 1.3$ channels in $\mathrm{Cabp2} 2^{\mathrm{LacZ} / \mathrm{LacZ}}$ IHCs}

Reconciling the in vitro IHC patch-clamp data with the in vivo SGN responses to sound (Figure 3.14), suggests that $\mathrm{Ca}_{V} 1.3$ channels in Cabp2-deficient IHCs undergo substantial steady-state inactivation already at the physiological resting membrane potential. This interpretation is partially supported by patch-clamp recordings that revealed a progressing decline in $\mathrm{Ca}^{2+}$ current amplitudes upon repetitive stimulation of Cabp2 $2^{\text {LacZ/LacZ }}$ IHCs and was already apparent within the first five depolarization pulses (Figure 3.11). The inactivated $\mathrm{Ca}_{V} 1.3$ channels also needed a prolonged time to recover (at $1 \mathrm{~s} 10 \% \mathrm{Ca}^{2+}$ current reduction).

Modulation of IHC $\mathrm{Ca}^{2+}$ channel function by various proteins was previously reported, for example for Bassoon/synaptic ribbon (Frank et al., 2010; Jing et al., 2013), RIMs (Jung et al., 2015b), and $\mathrm{Ca}^{2+}$ buffers (calbindin-28k, calretinin and parvalbumin- $\alpha$; (Pangršič et al., 2015)). Bassoon and/or the ribbon seem to be required to promote a large complement of $\mathrm{Ca}^{2+}$ channels at the $\mathrm{AZ}$ and affect their spatial organization. However, whether the effects of Bassoon disruption reflects the loss of direct regulation of the channels by Bassoon or the secondary loss of synaptic ribbons remains to be tested (Frank et al., 2010; Jing et al., 2013). RIMs were implicated in promoting $\mathrm{Ca}^{2+}$ channel abundance and clustering at the presynaptic $\mathrm{AZ}$, as shown in cultured hippocampal neurons (Graf et al., 2012), the calyx of Held (Han et al., 2011) and IHCs (Jung et al., 2015b). Despite of a decrease of $\mathrm{Ca}^{2+}$ current amplitudes (51.4\%) in IHCs of RIM1/2 double knock-out (DKO) mice, auditory signaling was only mildly affected (elevation of ABR-threshold: 
$16 \mathrm{~dB}$; (Jung et al., 2015b); as compared to Cabp2 $2^{\mathrm{LacZ} / \mathrm{LacZ}}: 30 \mathrm{~dB}$ ). $\mathrm{Ca}_{V} 1.3$ channels in mature IHCs are rather resistant to inactivation and show fast recovery (Johnson and Marcotti, 2008). These features might better support synaptic transmission in RIM1/2 DKO displaying a reduced $\mathrm{Ca}^{2+}$ channel number, than in Cabp $2^{\text {LacZ/LacZ }}$ IHCs with enhanced $\mathrm{Ca}_{V} 1.3$ inactivation. It seems as if a sole reduction of $\mathrm{Ca}^{2+}$ channel clusters at the active zone still suffices to mediate intact $\mathrm{Ca}^{2+}$ influx exocytosis coupling in the absence of RIM1/2. However, a stochastic loss of release sites due to $\mathrm{Ca}^{2+}$ channel inactivation on the other hand, might cause impaired sound encoding. Other difference between both genotypes are shown by SGN responses, revealing a good temporal precision of sound encoding in the absence of RIM1/2, but not in Cabp2 $2^{\text {LacZ/LacZ }}$ animals (Figure 3.14). The synchronous and delayed firing of SGNs is reflected in normal but slightly delayed ABR-wave I amplitudes in RIM1/2 DKO compared to the significant reduction and delay recorded in Cabp2-deficient mice (Figure 3.6). Whether the temporally imprecise sound evoked responses of SGNs is the sole consequence of presynaptic $\mathrm{Ca}_{V} 1.3$ channel dysfunction remains to be investigated. An IHC-specific conditional Cabp2 knock-out mouse could be used to exclude the contribution of SGN deficits and strengthen the hypothesis of $\mathrm{Ca}_{V} 1.3$ dysfunction as the underlying cause for DFNB93. In light of the fact that faithful sound encoding is preserved at reduced channel numbers (Jung et al., 2015b), the exact mechanism behind $\mathrm{Ca}^{2+}$ channel dysfunction inducing an increased steady-state remains elusive and certainly deserves further investigation.

Recently, an enhanced CDI was reported in triple $\mathrm{Ca}^{2+}$ buffer knock-out mice (calbindin-28k, calretinin and parvalbumin- $\alpha$; (Pangršič et al., 2015)), coinciding with increased fast $\mathrm{Ca}^{2+}$ influx (increase of approximately $20 \%$ ). $\mathrm{Ca}^{2+}$ influx-exocytosis coupling, addressing the readily-release vesicle pool was largely unchanged comparable to what I observed in Cabp2 $2^{\text {LacZ/LacZ }}$ animals (< 20 ms depolarizations; (Moser and Beutner, 2000); 3.12). Unexpectedly, in vivo SGN responses showed widely normal sound encoding properties under impaired $\mathrm{Ca}^{2+}$ buffer conditions (Pangršič et al., 2015), in contrast to Cabp2 $2^{\mathrm{LacZ} / \mathrm{LacZ}}$ animals with reduced onset- and adapted SGN firing rates. Moreover, SGNs exhibited higher spontaneous fiber responses, interpreted as increased presynaptic vesicle fusion as a consequence of a higher opening probability of $\mathrm{Ca}^{2+}$ channels as reported in (Robertson and Paki, 2002). In Cabp2-deficient SGNs I observed the opposite. Spontaneous firing rates were reduced, which might be explained by an accumulation of $\mathrm{Ca}^{2+}$ channels in the inactive state also at physiological resting membrane potential. The intact sound encoding in triple $\mathrm{Ca}^{2+}$ buffer knock-out animals on the other hand suggests more available $\mathrm{Ca}^{2+}$ channels due to the expression of a higher number at the $\mathrm{AZ}$ and potentially a faster recovery from inactivation of the $\mathrm{Ca}^{2+}$ channels compared to Cabp2 ${ }^{\mathrm{LacZ} / \mathrm{LacZ}}$ IHCs, but this hypothesis needs further testing. The exact mechanism behind the increased CDI observed in IHCs of mice lacking the three $\mathrm{Ca}^{2+}$ buffers remains to be determined. To date, no direct modulations of $\mathrm{Ca}_{V} 1.3$ by calbindin-28k, calretinin and parvalbumin- $\alpha$ were reported, therefore it is not known whether increased CDI is induced by direct interaction, or an auto-regulatory response of the channel due to elevated intra- 
cellular $\mathrm{Ca}^{2+}$ levels. If assumed that the observed increase in CDI of triple buffer knock-out mice is induced by indirect modulation of $\mathrm{Ca}_{V} 1.3$ channels, then the $\mathrm{Ca}_{V} 1.3$ channels might not be inactivated in a steady-state at physiological resting membrane potential as indicated by the high spontaneous SGN responses (Pangršič et al., 2015). An enhanced $\mathrm{Ca}^{2+}$ influx through a higher number of channels might be enough to drive faithful glutamate release. CaBP2 on the other hand is a direct modulator that keeps the $\mathrm{Ca}_{V} 1.3$ channel in a non-inactivated state. If one assumes, that $\mathrm{Ca}_{V} 1.3$ channels in $\mathrm{Cabp} 2^{\mathrm{LacZ} / \mathrm{LacZ}}$ mice are already inactivated at resting membrane potential, I should have found a smaller readily releasable pool in capacitance measurements, which was not observed. An unaffected readily release pool size was most likely permitted by the hyperpolarized recording conditions during these experiments. During patch-clamp recordings IHCs are typically held at relatively hyperpolarized potentials between consecutive stimuli (e.g. -70 to $-90 \mathrm{mV}$ ) this might relieve the inactivation of $\mathrm{Ca}_{V} 1.3$ channels in Cabp $2^{\text {LacZ/LacZ }}$ IHCs masking steady-state inactivation of $\mathrm{Ca}_{V} 1.3$ channels under in vitro conditions. I tried to more faithfully reproduce the presumed in vivo steady-state $\mathrm{Ca}^{2+}$ channel inactivation in Cabp2 $2^{\text {LacZ/LacZ }}$ IHCs by train recordings at more depolarized holding potentials. However, the expected $\mathrm{V}_{\text {holding }}$ dependent increase (-69 mV vs. $-59 \mathrm{mV}$; Figure 3.11) in sustained inactivation was not observed. It needs to be mentioned that the estimates of the physiological resting membrane potential are controversial, ranging form $-75 \mathrm{mV}$ in mature (Glowatzki and Fuchs, 2002) to $-59 \mathrm{mV}$ in immature IHCs (Johnson et al., 2011) and might also be temperature dependent (-58 $\mathrm{mV}$ at body temperature (Johnson et al., 2011)) with further indications that physiologically, the resting membrane potential of IHCs might even be higher. Therefore, to further strengthen my hypothesis of steady-state inactivation in vitro recordings at body temperature and even more depolarized $\mathrm{V}_{\text {holding }}$ might reveal the expected effect considering that CDI is temperature dependent (Grant and Fuchs, 2008).

\subsection{Cabp2 acts as a $\mathrm{Ca}^{2+}$ buffer in IHCs}

My data suggest that $\mathrm{Cabp} 2$ is not only a $\mathrm{Ca}^{2+}$ sensing molecule, which regulates $\mathrm{Ca}^{2+}$ channels but also contributes to $\mathrm{Ca}^{2+}$ buffering in IHCs. IHCs are critically dependent on endogenous $\mathrm{Ca}^{2+}$ binding proteins to spatially confine $\mathrm{Ca}^{2+}$ influx to the site of release by rapidly removing free $\mathrm{Ca}^{2+}$ ions from the point of entry (Roberts, 1993). The hypothesis that Cabp2 contributes to endogenous $\mathrm{Ca}^{2+}$ buffering in IHCs arose from presynaptic membrane capacitance recordings, which revealed an age-dependent enhancement of efficiency of $\mathrm{Ca}^{2+}$ influx to elicit sustained exo-

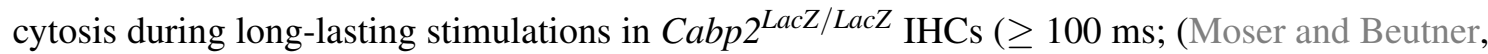
2000); Figure 3.12B). This phenomenon was even more apparent when applying ten consecutive depolarizing pulses during recordings in 5-week-old Cabp $2^{\mathrm{LacZ} / \mathrm{LacZ}}$ animals (individual depolarization of $100 \mathrm{~ms}$ ), resulting in a two-fold increase in $\Delta \mathrm{C}_{m}$, while $\mathrm{Q}_{C a}$ was decreased by $25 \%$ compared to Cabp2 $2^{+/+}$IHCs (Figure 3.13).

The shift in sustained release upon age shows that the effect of Cabp2 on vesicular release is 
more pronounced at higher age and suggests an age-dependent change in relative expression of Cabp2, Cabp1, and Cabp4 in IHCs. In general, this hypothesis could be tested by quantitative immunostainings, which was not feasible in this study due to the lack of suitable antibodies. An alternative approach is quantitative RT-PCR of Cabp $2^{\mathrm{LacZ} / \mathrm{LacZ}}$ and control IHCs to get an impression of age-dependent changes in Cabp (Cabp1, Cabp2, Cabp4) mRNA expression.

However, the increase in sustained exocytosis initially seemed unexpected and counter-intuitive, considering a reduction of available $\mathrm{Ca}^{2+}$ channels at the $\mathrm{AZ}$ due to increased voltage-dependent inactivation of $\mathrm{Ca}_{V} 1.3$ channels. The excessive sustained exocytosis under reduced $\mathrm{Ca}^{2+}$ buffer conditions might be caused by an accelerated $\mathrm{Ca}^{2+}$ dependent recruitment of vesicles as shown in turtles (Schnee et al., 2011) or by enhanced parallel extrasynaptic vesicle fusion as reported for $\mathrm{Ca}^{2+}$ buffer-deficient IHCs in mice (Pangršič et al., 2015).

Notably, in hair cells of frogs, turtles and mice, high concentrations of mobile EF-hand $\mathrm{Ca}^{2+}$ chelators were estimated to provide $\mathrm{Ca}^{2+}$ binding sides in the mM-range (Pangršič et al., 2015; Ricci et al., 1998; Roberts, 1993). At ribbon synapses of IHCs, the postulated function of these mobile $\mathrm{Ca}^{2+}$ binding proteins is the spatio-temporally confined shaping of the presynaptic $\mathrm{Ca}^{2+}$ signaling (Frank et al., 2009; Issa and Hudspeth, 1996; Pangršič et al., 2015; Roberts, 1993). Recently, the role of three EF-hand $\mathrm{Ca}^{2+}$ binding proteins calbindin-28k, calretinin and parvalbumin$\alpha$ was investigated, using a triple knock-out mouse model (Pangršič et al., 2015). These triple $\mathrm{Ca}^{2+}$ buffer knock-out mice exhibited excessive exocytosis upon prolonged depolarization $(\geq 100$ $\mathrm{ms}$ ), comparable to what was observed in Cabp $2^{\mathrm{LacZ} / \mathrm{LacZ}} \mathrm{IHCs}$. Surprisingly, in contrast in vivo SGN responses to sound stimulation showed normal adapted firing rates. These SGN responses, monitored by extracellular recordings in vivo report the spiking behavior in response to presynaptic vesicular release at a single IHC AZ (Buran et al., 2010). Pangršič and colleagues therefore hypothesised that the observed excessive vesicle fusion does not happen at the AZ, but rather extrasynaptically due to an increased $\mathrm{Ca}^{2+}$ spread in the absence of the three buffers. In this study, this hypothesis was additionally supported by mathematical modeling of the effect of $\mathrm{Ca}^{2+}$ buffering on the coupling distance of vesiclular release. Based on the previous observation, I tested whether Cabp2 buffers free $\mathrm{Ca}^{2+}$ ions in IHCs by $\mathrm{Ca}^{2+}$ buffer substitution experiments (Figure 3.13) and showed that both, excessive exocytosis and $\mathrm{Ca}^{2+}$ influx $\left(\mathrm{Q}_{C a}\right)$ were statistically indistinguishable in $\mathrm{Cabp2}^{+/+}$and Cabp2 $2^{\mathrm{LacZ} / \mathrm{LacZ}}$ IHCs (Figure 3.13) when aiming to induce comparable intracellular synthetic $\mathrm{Ca}^{2+}$ buffer concentrations. While the waiting time of $5 \mathrm{~min}$ prior to stimulation should provide sufficient wash-out of mobile $\mathrm{Ca}^{2+}$ buffers, there might still have been some more $\mathrm{Ca}^{2+}$ binding sites and even Cabp2 left due to constitutive binding of $\mathrm{CaBPs}$ to the $\mathrm{Ca}_{V} 1.3$ channel under $\mathrm{Ca}^{2+}$ free conditions (Findeisen et al., 2013) and membrane anchoring of CaBP2. However, this would be expected to preserve the difference between IHCs of both genotypes. With this experimental design, one cannot rule out that additional functions of CaBP2 may have been concealed masking the postulated role as $\mathrm{Ca}^{2+}$ buffer, if $\mathrm{CaBP} 2$ was indeed lost due to the wash-out 
in $\mathrm{Cabp}^{+/+}$IHCs.

If one assumed that excess sustained exocytosis found in the absence of CaBP2 occurred synaptically, extracellular in vivo recordings of SGN spiking behavior should reveal increased adapted firing rates in $C a b p 2^{\mathrm{LacZ} / \mathrm{LacZ}}$ animals. This was not observed, and in line with the findings of Pangršič and colleagues (Pangršič et al., 2015), I suggest that the reduction in $\mathrm{Ca}^{2+}$ buffering upon disruption of Cabp2 enables excess extrasynaptic fusion. Since ABR thresholds were normal in triple buffer knock-out mice (Pangršič et al., 2015), impaired $\mathrm{Ca}^{2+}$ buffering in Cabp $2^{\mathrm{LacZ} / \mathrm{LacZ}}$ IHC is most probably not the underlying cause for the hearing impairment observed in Cabp2 $2^{\text {LacZ/LacZ }}$ mice and human DFNB93 patients.

In future, experiments are required to further strengthen the $\mathrm{Ca}^{2+}$ buffer hypothesis of $\mathrm{CaBP} 2$, including the determination of $\mathrm{Ca}^{2+}$ binding kinetics of $\mathrm{CaBP} 2$. So far, a detailed analysis is missing for $\mathrm{CaBP} 2$ and initial measurements of steady-state $\mathrm{Ca}^{2+}$ binding properties by isothermal titration calorimetry suggest relatively low affinity binding of $\mathrm{Ca}^{2+}$ (Meese et al., unpublished).In addition, an enhanced $\mathrm{Ca}^{2+}$ spread can be identified by $\mathrm{Ca}^{2+}$ imaging of re-patched IHCs after short loading periods with a $\mathrm{Ca}^{2+}$ dye. However, these experiments are difficult to implement, due to the necessity of re-patching IHCs of older animals under perforated-patch configuration to prevent wash-out of $\mathrm{CaBP} 2$.

To date, one function reported for $\mathrm{CaBP} 1$, namely the inhibition of $\mathrm{Ca}^{2+}$ release from intracellular $\mathrm{Ca}^{2+}$ stores (CICR) has been reported, that also supports the idea of extrasynaptic vesicle fusion due to elevated intracellular $\mathrm{Ca}^{2+}$ levels (Kasri et al., 2004; Li et al., 2013). Transient expression experiments in chinese hamster ovary cells (Kasri et al., 2004) and neuroendocrine PC12 cells (Haynes et al., 2004) indicated that CaBP1 inhibits CICR by modulating inositol 1,4,5 triphosphate receptors (IP3 receptor). However, this hypothesis is unlikely to apply to mature IHCs given multiple considerations: (i) CICR was only reported in immature IHCs (Frank et al., 2009; Kennedy and Meech, 2002; Neef et al., 2009) (ii) where capacitance recordings after pharmacological enhancement of CICR failed to influence vesicular release (Iosub et al., 2015), and (iii) CICR in immature IHCs is mediated by ryanodine receptors (Grant et al., 2006) which were shown to be inhibited by CaM but not CaBP1 (Kasri et al., 2004). Future experiments should investigate if Cabp2 modulates ryanodine receptors and causes the apparent lack of CICR in mature IHCs (Frank et al., 2009).

Apart from the regulation of CICR by CaBP1, no other functions of CaBPs were reported that could explain my observations. Therefore, I favor the hypothesis that, in addition to direct $\mathrm{Ca}_{V} 1.3$ regulation, Cabp2 buffers elevated intracellular $\mathrm{Ca}^{2+}$ levels and constrains $\mathrm{Ca}^{2+}$ signaling to the AZ of IHC ribbon synapses. 


\subsection{DFNB93 as another candidate of a channelopathy}

After an extensive investigation of the Cabp2-deficient mouse model I observed an increase in voltage-dependent steady-state inactivation and delayed recovery from inactivation of $\mathrm{Ca}_{V} 1.3$ channels at the IHC ribbon synapse as candidate disease mechanism causing DFNB93. IHC synapses appeared normally developed and were present at normal numbers arguing against synap-

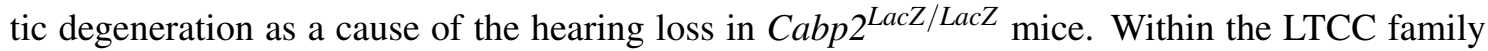
mutations affecting $\mathrm{Ca}^{2+}$ channel inactivation were reported to cause either a reduction (Splawski et al., 2004, 2005) or an increase (Kraus et al., 1998, 2000) of inactivation. A mutation in the pore forming S6 segment of the $\mathrm{Ca}_{V} 2.1 \alpha 1$ subunit displays a similar channel dysfunction as observed for $\mathrm{Ca}_{V} 1.3$ in the absence of Cabp2. In a transient expression system the VDI was increased and coincides with prolonged recovery times (Kraus et al., 1998). This channelopathy was postulated as the underlying cause for familial hemiplegic migraine. As already mentioned in (Section 1.4.3), to date only one human mutations was reported to impair $\mathrm{Ca}_{V} 1.3$ function by compromising ionconductance of the $\mathrm{Ca}^{2+}$ channel resulting in SANDD syndrome. My data suggests that the synaptic impairment DFNB93 could count as another $\mathrm{Ca}_{V} 1.3$ channelopathy. This idea is supported by the fact that CaBPs were shown to be constitutively bound to the $\mathrm{Ca}_{V} \alpha 1$ subunit (Findeisen et al., 2013; Zhou et al., 2004) and were classified comparable to calmodulin (Pitt, 2007) as subunit of LTCCs in CaBP expressing cells (Minor and Findeisen, 2010).

\subsection{Potential of virus mediated gene therapy to treat DFNB93}

Recently, virus mediated gene therapeutic approaches have become powerful tools to restore hearing (Akil et al., 2012; Moser, 2015). To test whether $\mathrm{Ca}_{V}$ 1.3-dysfunction can be restored by a gene therapeutic approach, I designed an AAV2/1 virus, where CABP2-wt was C-terminally fused to EGFP. This viral construct revealed a high IHC transduction efficiency $(88.8 \%)$ and cellular specificity, in line with previous observations (Bedrosian et al., 2006; Jung et al., 2015a; Reisinger et al., 2011). Importantly, Cabp2-EGFP overexpression in wild-type IHCs did not induce any pathological effects on hearing.

Surprisingly, viral transduction of $C a b p 2^{\text {LacZ/LacZ }}$ animals failed to restore hearing, shown by comparable ABR wave I amplitudes and ABR thresholds (Figure 3.17). While Cabp2 mediated inhibition of $\mathrm{Ca}_{V} 1.3$ inactivation was observed in AAV-transduced ipsilateral Cabp2 $2^{\text {LacZ/LacZ }} \mathrm{IHCs}$ (Figure 3.17), sustained exocytosis, on the other hand, was reduced which seems in-line with the proposed function of $\mathrm{CaBP} 2$ as a $\mathrm{Ca}^{2+}$ buffer (see above). There are at least two non-exclusive explanations for the dysfunction of the Cabp2-EGFP fusion protein in IHCs: (i) the expressed fusion-protein fails to bind to the $\mathrm{Ca}_{V} 1.3$ channel due to the presence of other $\mathrm{Ca}_{V} 1.3$ interacting proteins in IHCs, which are not expressed upon heterologous expression, or (ii) the C-terminal EGFP-tag of Cabp2 provides a disadvantage for Cabp2 in competing with $\mathrm{CaM}$ for the binding 
to the IQ domain of the $\mathrm{Ca}_{V} \alpha 1$ subunit. Therefore, the regulation of VDI by CaBP2 might be compromised.

However, for this particular project a direct readout of $\mathrm{CaBP} 2$ was required, due to the lack of CaBP2 specific antibodies. Therefore, I did not use a viral construct containing an internal ribosomes entry site (IRES) for a separate EGFP translation due to an indirect readout of Cabp2 expression level. The fluorescent signal intensity would not reliably inform about Cabp2 expression in IHCs as needed for patch-clamp recordings. One way to preserve the report of Cabp2 expression level is to insert a self-cleaving P2A peptide sequence between the Cabp2 and EGFP sequence. In the presence of that sequence ribosomes were suggested to skip the synthesis of the C-terminal peptide bond of $2 \mathrm{~A}$ peptides resulting in the expression of two separate proteins (Donnelly et al., 2001). The specific advantage of the P2A peptide is a high self-cleaving efficiency as proposed recently (Kim et al., 2011).

Whether virus-mediated gene transfer is an option to treat DFNB93 patients remains an open question that should be addressed in future. The recessive character of DFNB93 supports the idea that this gene therapeutic approach is a valuable method to restore hearing in these patients. The fact that developmental deficits were not observed in Cabp2 $2^{\text {LacZ/LacZ }}$ IHCs suggest that virus-mediated hearing restoration could also be applicable at higher age, if the longevity of the virus expression is granted. Previous studies on virus-mediated gene transfer involved hearing restoration, which was successful in Vglut3 knock-out mice (Akil et al., 2012), Tmc1 mutant mice ((Askew et al., 2015); Section 1.5) and AP-2 $\mu$ mutants (Jung et al., 2015a). Post-natal injections at higher age (p8) were shown to partially compromise the longevity of the hearing restoration in Vglut3 knock-out mice (hearing restoration $<7$ weeks), which display profound hearing impairment (>100 db threshold elevation; (Ruel et al., 2008)). Therefore future work needs to address the optimal time point for virus injection to ensure efficient and long-lasting gene replacement by viral vectors. 


\section{Summary}

$\mathrm{Ca}_{V} 1.3 \mathrm{Ca}^{2+}$ channels are key elements of faithful sound encoding at IHC ribbon synapses. In order to indefatigably respond to receptor potentials, $\mathrm{Ca}_{V} 1.3$ channels of IHCs activate at negative potentials and exhibit weak inactivation. The mechanisms underlying this mild negative feedback to $\mathrm{Ca}^{2+}$ influx in IHCs are not well understood. CaBPs are candidate proteins shown to prevent inactivation and stabilize the opening of the $\mathrm{Ca}^{2+}$ channel. Dysfunction of CaBPs can cause impairment of vision and hearing. The recent discovery of the deafness locus DFNB93 and identification of a mutation in $C A B P 2$ as underlying cause for hearing impairment in human patients was the starting point of my work. I investigated the role of $\mathrm{CaBP} 2$ in hearing and, in particular addressed the question, whether $\mathrm{CaBP} 2$ contributes to the inhibition of $\mathrm{Ca}_{V} 1.3$ channels inactivation in IHCs. The regulation of the $\mathrm{Ca}_{V} 1.3$ channel was probed in heterologous expression system. CaBP2 was genetically disrupted and (immune)histochemistry, patch-clamp recordings and in vivo physiology were combined on Cabp2 $2^{\mathrm{LacZ} / \mathrm{LacZ}}$ mice to elucidate the function of CaBP2 and to unravel the disease mechanism of DFNB93.

I introduced HEK293/hSK3-1 cells as valuable tool to investigate $\mathrm{Ca}_{V} 1.3$ function. $\mathrm{Ca}_{V} 1.3$ channel modulation by wild-type $\mathrm{CABP} 2$ and the disease causing $\mathrm{CABP} 2-\mathrm{EF}^{\Delta 3 / 4}$ mutant revealed two candidate mechanisms of DFNB93. First, the CABP2-EF ${ }^{43 / 4}$ shifted the voltage-sensitivity of $\mathrm{Ca}_{V} 1.3$ channels out of the presumptive IHC receptor potentials. Second, CABP2-EF ${ }^{\Delta 3 / 4}$ via failure to inhibit inactivation might cause enhanced steady-state inactivation leading to fewer $\mathrm{Ca}_{V} 1.3$ channels available for activation.

A detailed investigation of hearing at a system's level showed that $C a b p 2^{\text {LacZ/LacZ }}$ mice exhibited progressive hearing loss due to an auditory synaptopathy at the IHC ribbon synapse. At the IHC level the degree of voltage-dependent inactivation was increased, while the operating range of $\mathrm{Ca}_{V} 1.3 \mathrm{Ca}^{2+}$ channels was unchanged upon CaBP2-deficiency. As a consequence, IHC $\mathrm{Ca}_{V} 1.3$ channels instantaneously accumulated in an inactivated state upon train stimulation and required a prolonged time to recover, suggesting that the $\mathrm{Ca}^{2+}$ channels undergo a steady-state inactivation in the absence of Cabp2. Under the tested recording conditions hearing impairment progression does not coincide with an increase of $\mathrm{Ca}^{2+}$ current inactivation without an indication of a degenerative hearing loss. In parallel, in vivo SGN responses of Cabp2 $2^{\mathrm{LaCZ} / \mathrm{LacZ}}$ mice exhibited a reduction in onset and adapted firing rates and impaired temporal precision of sound encoding. Reduced rate and temporal precision of firing account for the observed reduction and delay of ABR-wave I amplitudes and the ABR-threshold increase. In Cabp2 $2^{\text {LacZ/LacZ }}$ IHCs of older mice sustained exocystosis exceeded $\mathrm{Cabp} 2^{+/+}$control levels while $\mathrm{Ca}^{2+}$ influx was reduced. Endogenous $\mathrm{Ca}^{2+}$ buffer substitution experiments with synthetic $\mathrm{Ca}^{2+}$ chelators showed a reversibility of sustained exocytosis under equalized $\mathrm{Ca}^{2+}$ buffer conditions, indicating impaired $\mathrm{Ca}^{2+}$ buffering 
upon Cabp2-deficiency. However, a parallel increase in the adapted firing rates of SGNs was not observed, suggesting that excess exocytosis does not contribute to sound encoding but mostly occurs extrasynaptically.

AAV2/1 mediated gene transfer of Cabp2-EGFP revealed high transduction efficiency of IHCs, without affecting hearing of wild-type mice, however failed to improve ABR-thresholds in Cabp2deficient animals. An alternative design of the viral construct might be required for effective virus-mediated gene therapy of the hearing impairment DFNB93. 


\section{Bibliography}

\section{References}

WHO Deafness and hearing loss. http://www . who.int/mediacentre/factsheets/fs300/ en/. Accessed: 2015-09-09.

M. K. Ahlijanian, R. E. Westenbroek, and W. A. Catterall. Subunit structure and localization of dihydropyridine-sensitive calcium channels in mammalian brain, spinal cord, and retina. Neuron, 4(6):819-832, Jun 1990.

Z. M. Ahmed, R. Goodyear, S. Riazuddin, A. Lagziel, P. K. Legan, M. Behra, S. M. Burgess, K. S. Lilley, E. R. Wilcox, S. Riazuddin, A. J. Griffith, G. I. Frolenkov, I. A. Belyantseva, G. P. Richardson, and T. B. Friedman. The tip-link antigen, a protein associated with the transduction complex of sensory hair cells, is protocadherin-15. J. Neurosci., 26(26):7022-7034, Jun 2006.

O. Akil, R. P. Seal, K. Burke, C. Wang, A. Alemi, M. During, R. H. Edwards, and L. R. Lustig. Restoration of hearing in the VGLUT3 knockout mouse using virally mediated gene therapy. Neuron, 75(2):283-293, Jul 2012.

J. F. Ashmore. A fast motile response in guinea-pig outer hair cells: the cellular basis of the cochlear amplifier. J. Physiol. (Lond.), 388:323-347, Jul 1987.

C. Askew, C. Rochat, B. Pan, Y. Asai, H. Ahmed, E. Child, B. L. Schneider, P. Aebischer, and J. R. Holt. Tmc gene therapy restores auditory function in deaf mice. Sci Transl Med, 7(295): 295ra108, Jul 2015.

S. M. Baig, A. Koschak, A. Lieb, M. Gebhart, C. Dafinger, G. Nurnberg, A. Ali, I. Ahmad, M. J. Sinnegger-Brauns, N. Brandt, J. Engel, M. E. Mangoni, M. Farooq, H. U. Khan, P. Nurnberg, J. Striessnig, and H. J. Bolz. Loss of Ca(v)1.3 (CACNA1D) function in a human channelopathy with bradycardia and congenital deafness. Nat. Neurosci., 14(1):77-84, Jan 2011.

J. C. Bedrosian, M. A. Gratton, J. V. Brigande, W. Tang, J. Landau, and J. Bennett. In vivo delivery of recombinant viruses to the fetal murine cochlea: transduction characteristics and long-term effects on auditory function. Mol. Ther., 14(3):328-335, Sep 2006.

M. Ben-Johny and D. T. Yue. Calmodulin regulation (calmodulation) of voltage-gated calcium channels. J. Gen. Physiol., 143(6):679-692, Jun 2014.

G. M. Bernstein and O. T. Jones. Kinetics of internalization and degradation of N-type voltagegated calcium channels: role of the alpha2/delta subunit. Cell Calcium, 41(1):27-40, Jan 2007.

L. Berrou, G. Bernatchez, and L. Parent. Molecular determinants of inactivation within the I-II linker of alpha1E (CaV2.3) calcium channels. Biophys. J., 80(1):215-228, Jan 2001. 
D. Bichet, V. Cornet, S. Geib, E. Carlier, S. Volsen, T. Hoshi, Y. Mori, and M. De Waard. The I-II loop of the $\mathrm{Ca} 2+$ channel alpha1 subunit contains an endoplasmic reticulum retention signal antagonized by the beta subunit. Neuron, 25(1):177-190, Jan 2000.

J. L. Black. The voltage-gated calcium channel gamma subunits: a review of the literature. $J$. Bioenerg. Biomembr., 35(6):649-660, Dec 2003.

G. Bock, M. Gebhart, A. Scharinger, W. Jangsangthong, P. Busquet, C. Poggiani, S. Sartori, M. E. Mangoni, M. J. Sinnegger-Brauns, S. Herzig, J. Striessnig, and A. Koschak. Functional properties of a newly identified C-terminal splice variant of Cav1.3 L-type Ca2+ channels. J. Biol. Chem., 286(49):42736-42748, Dec 2011.

A. Brandt, J. Striessnig, and T. Moser. CaV1.3 channels are essential for development and presynaptic activity of cochlear inner hair cells. J. Neurosci., 23(34):10832-10840, Nov 2003.

S. Brunet, T. Scheuer, and W. A. Catterall. Cooperative regulation of $\mathrm{Ca}(\mathrm{v}) 1.2$ channels by intracellular $\operatorname{Mg}(2+)$, the proximal C-terminal EF-hand, and the distal C-terminal domain. J. Gen. Physiol., 134(2):81-94, Aug 2009.

A. H. Bunt. Enzymatic digestion of synaptic ribbons in amphibian retinal photoreceptors. Brain Res., 25(3):571-577, Feb 1971.

Z. Buraei and J. Yang. The beta subunit of voltage-gated Ca2+ channels. Physiol. Rev., 90(4): 1461-1506, Oct 2010.

B. N. Buran, N. Strenzke, A. Neef, E. D. Gundelfinger, T. Moser, and M. C. Liberman. Onset coding is degraded in auditory nerve fibers from mutant mice lacking synaptic ribbons. $J$. Neurosci., 30(22):7587-7597, Jun 2010.

W. A. Catterall. Voltage-gated calcium channels. Cold Spring Harb Perspect Biol, 3(8):a003947, Aug 2011.

D. Cosgrove, J. M. Kornak, and G. Samuelson. Expression of basement membrane type IV collagen chains during postnatal development in the murine cochlea. Hear. Res., 100(1-2):21-32, Oct 1996.

P. J. Coucke, P. Van Hauwe, P. M. Kelley, H. Kunst, I. Schatteman, D. Van Velzen, J. Meyers, R. J. Ensink, M. Verstreken, F. Declau, H. Marres, K. Kastury, S. Bhasin, W. T. McGuirt, R. J. Smith, C. W. Cremers, P. Van de Heyning, P. J. Willems, S. D. Smith, and G. Van Camp. Mutations in the KCNQ4 gene are responsible for autosomal dominant deafness in four DFNA2 families. Hum. Mol. Genet., 8(7):1321-1328, Jul 1999. 
C. W. Cremers, R. F. Plantinga, and H. Kremer. [From gene to disease; DFNA8/12, an autosomal dominant inherited bowl-shaped sensorineural hearing impairment]. Ned Tijdschr Geneeskd, 151(9):531-534, Mar 2007.

G. Cui, A. C. Meyer, I. Calin-Jageman, J. Neef, F. Haeseleer, T. Moser, and A. Lee. Ca2+binding proteins tune Ca2+-feedback to Cav1.3 channels in mouse auditory hair cells. J. Physiol. (Lond.), 585(Pt 3):791-803, Dec 2007.

O. Dafi, L. Berrou, Y. Dodier, A. Raybaud, R. Sauve, and L. Parent. Negatively charged residues in the N-terminal of the AID helix confer slow voltage dependent inactivation gating to CaV1.2. Biophys. J., 87(5):3181-3192, Nov 2004.

P. Dallos. The Cochlea, an Overview in Cochlea Neurobiology. Springer-Verlag, New York, 1996. ISBN 9780387944494.

P. Dallos and B. Fakler. Prestin, a new type of motor protein. Nat. Rev. Mol. Cell Biol., 3(2): 104-111, Feb 2002.

A. Davies, I. Kadurin, A. Alvarez-Laviada, L. Douglas, M. Nieto-Rostro, C. S. Bauer, W. S. Pratt, and A. C. Dolphin. The alpha2delta subunits of voltage-gated calcium channels form GPIanchored proteins, a posttranslational modification essential for function. Proc. Natl. Acad. Sci. U.S.A., 107(4):1654-1659, Jan 2010.

O. Dick, S. tom Dieck, W. D. Altrock, J. Ammermuller, R. Weiler, C. C. Garner, E. D. Gundelfinger, and J. H. Brandstatter. The presynaptic active zone protein bassoon is essential for photoreceptor ribbon synapse formation in the retina. Neuron, 37(5):775-786, Mar 2003.

A. C. Dolphin. The alpha2delta subunits of voltage-gated calcium channels. Biochim. Biophys. Acta, 1828(7):1541-1549, Jul 2013.

M. L. Donnelly, G. Luke, A. Mehrotra, X. Li, L. E. Hughes, D. Gani, and M. D. Ryan. Analysis of the aphthovirus $2 \mathrm{~A} / 2 \mathrm{~B}$ polyprotein 'cleavage' mechanism indicates not a proteolytic reaction, but a novel translational effect: a putative ribosomal 'skip'. J. Gen. Virol., 82(Pt 5):1013-1025, May 2001.

H. Dou, A. E. Vazquez, Y. Namkung, H. Chu, E. L. Cardell, L. Nie, S. Parson, H. S. Shin, and E. N. Yamoah. Null mutation of alpha1D Ca2+ channel gene results in deafness but no vestibular defect in mice. J. Assoc. Res. Otolaryngol., 5(2):215-226, Jun 2004.

F. J. Dreiling, M. M. Henson, and O. W. Henson. The presence and arrangement of type II collagen in the basilar membrane. Hear. Res., 166(1-2):166-180, Apr 2002. 
J. Engel, C. Braig, L. Ruttiger, S. Kuhn, U. Zimmermann, N. Blin, M. Sausbier, H. Kalbacher, S. Munkner, K. Rohbock, P. Ruth, H. Winter, and M. Knipper. Two classes of outer hair cells along the tonotopic axis of the cochlea. Neuroscience, 143(3):837-849, Dec 2006.

G. C. Faas, S. Raghavachari, J. E. Lisman, and I. Mody. Calmodulin as a direct detector of Ca2+ signals. Nat. Neurosci., 14(3):301-304, Mar 2011.

G. Ferreira, J. Yi, E. Rios, and R. Shirokov. Ion-dependent inactivation of barium current through L-type calcium channels. J. Gen. Physiol., 109(4):449-461, Apr 1997.

A. P. Few, N. J. Lautermilch, R. E. Westenbroek, T. Scheuer, and W. A. Catterall. Differential regulation of $\mathrm{CaV} 2.1$ channels by calcium-binding protein 1 and visinin-like protein-2 requires N-terminal myristoylation. J. Neurosci., 25(30):7071-7080, Jul 2005.

F. Findeisen and D. L. Minor. Structural basis for the differential effects of CaBP1 and calmodulin on $\mathrm{Ca}(\mathrm{V}) 1.2$ calcium-dependent inactivation. Structure, 18(12):1617-1631, Dec 2010.

F. Findeisen, C. H. Rumpf, and D. L. Minor. Apo states of calmodulin and CaBP1 control CaV1 voltage-gated calcium channel function through direct competition for the IQ domain. J. Mol. Biol., 425(17):3217-3234, Sep 2013.

T. Frank, D. Khimich, A. Neef, and T. Moser. Mechanisms contributing to synaptic Ca2+ signals and their heterogeneity in hair cells. Proc. Natl. Acad. Sci. U.S.A., 106(11):4483-4488, Mar 2009.

T. Frank, M. A. Rutherford, N. Strenzke, A. Neef, T. Pangršič, D. Khimich, A. Fejtova, A. Fetjova, E. D. Gundelfinger, M. C. Liberman, B. Harke, K. E. Bryan, A. Lee, A. Egner, D. Riedel, and T. Moser. Bassoon and the synaptic ribbon organize $\mathrm{Ca} 2+$ channels and vesicles to add release sites and promote refilling. Neuron, 68(4):724-738, Nov 2010.

G. I. Frolenkov, M. Atzori, F. Kalinec, F. Mammano, and B. Kachar. The membrane-based mechanism of cell motility in cochlear outer hair cells. Mol. Biol. Cell, 9(8):1961-1968, Aug 1998.

P. A. Fuchs. Time and intensity coding at the hair cell's ribbon synapse. J. Physiol. (Lond.), 566 (Pt 1):7-12, Jul 2005.

B. Gao, Y. Sekido, A. Maximov, M. Saad, E. Forgacs, F. Latif, M. H. Wei, M. Lerman, J. H. Lee, E. Perez-Reyes, I. Bezprozvanny, and J. D. Minna. Functional properties of a new voltagedependent calcium channel alpha(2)delta auxiliary subunit gene (CACNA2D2). J. Biol. Chem., 275(16):12237-12242, Apr 2000.

J. A. Garcia, A. G. Yee, P. G. Gillespie, and D. P. Corey. Localization of myosin-Ibeta near both ends of tip links in frog saccular hair cells. J. Neurosci., 18(21):8637-8647, Nov 1998. 
M. Gebhart, G. Juhasz-Vedres, A. Zuccotti, N. Brandt, J. Engel, A. Trockenbacher, G. Kaur, G. J. Obermair, M. Knipper, A. Koschak, and J. Striessnig. Modulation of Cav1.3 Ca2+ channel gating by Rab3 interacting molecule. Mol. Cell. Neurosci., 44(3):246-259, Jul 2010.

G. S. Geleoc and J. R. Holt. Sound strategies for hearing restoration. Science, 344(6184):1241062, May 2014.

E. Glowatzki and P. A. Fuchs. Transmitter release at the hair cell ribbon synapse. Nat. Neurosci., 5(2):147-154, Feb 2002.

R. J. Goodyear and G. P. Richardson. Extracellular matrices associated with the apical surfaces of sensory epithelia in the inner ear: molecular and structural diversity. J. Neurobiol., 53(2): 212-227, Nov 2002.

E. R. Graf, V. Valakh, C. M. Wright, C. Wu, Z. Liu, Y. Q. Zhang, and A. DiAntonio. RIM promotes calcium channel accumulation at active zones of the Drosophila neuromuscular junction. $J$. Neurosci., 32(47):16586-16596, Nov 2012.

E. Grandi, S. Morotti, K. S. Ginsburg, S. Severi, and D. M. Bers. Interplay of voltage and Cadependent inactivation of L-type Ca current. Prog. Biophys. Mol. Biol., 103(1):44-50, Sep 2010.

L. Grant and P. Fuchs. Calcium- and calmodulin-dependent inactivation of calcium channels in inner hair cells of the rat cochlea. J. Neurophysiol., 99(5):2183-2193, May 2008.

L. Grant, S. Slapnick, H. Kennedy, and C. Hackney. Ryanodine receptor localisation in the mammalian cochlea: an ultrastructural study. Hear. Res., 219(1-2):101-109, Sep 2006.

C. W. Graydon, S. Cho, G. L. Li, B. Kachar, and H. von Gersdorff. Sharp Ca2+ nanodomains beneath the ribbon promote highly synchronous multivesicular release at hair cell synapses. $J$. Neurosci., 31(46):16637-16650, Nov 2011.

C. W. Graydon, J. Zhang, N. W. Oesch, A. A. Sousa, R. D. Leapman, and J. S. Diamond. Passive diffusion as a mechanism underlying ribbon synapse vesicle release and resupply. J. Neurosci., 34(27):8948-8962, Jul 2014.

F. D. Gregory, T. Pangršič, I. E. Calin-Jageman, T. Moser, and A. Lee. Harmonin enhances voltage-dependent facilitation of Cav1.3 channels and synchronous exocytosis in mouse inner hair cells. J. Physiol. (Lond.), 591(Pt 13):3253-3269, Jul 2013.

R. Gueta, E. Tal, Y. Silberberg, and I. Rousso. The 3D structure of the tectorial membrane determined by second-harmonic imaging microscopy. J. Struct. Biol., 159(1):103-110, Jul 2007. 
A. W. Gummer, W. Hemmert, and H. P. Zenner. Resonant tectorial membrane motion in the inner ear: its crucial role in frequency tuning. Proc. Natl. Acad. Sci. U.S.A., 93(16):8727-8732, Aug 1996.

F. Haeseleer, I. Sokal, C. L. Verlinde, H. Erdjument-Bromage, P. Tempst, A. N. Pronin, J. L. Benovic, R. N. Fariss, and K. Palczewski. Five members of a novel $\mathrm{Ca}(2+)$-binding protein (CABP) subfamily with similarity to calmodulin. J. Biol. Chem., 275(2):1247-1260, Jan 2000.

F. Haeseleer, Y. Imanishi, T. Maeda, D. E. Possin, A. Maeda, A. Lee, F. Rieke, and K. Palczewski. Essential role of $\mathrm{Ca} 2+-$ binding protein 4, a Cav1.4 channel regulator, in photoreceptor synaptic function. Nat. Neurosci., 7(10):1079-1087, Oct 2004.

Y. Han, P. S. Kaeser, T. C. Sudhof, and R. Schneggenburger. RIM determines Ca2+ channel density and vesicle docking at the presynaptic active zone. Neuron, 69(2):304-316, Jan 2011.

M. Hans, S. Luvisetto, M. E. Williams, M. Spagnolo, A. Urrutia, A. Tottene, P. F. Brust, E. C. Johnson, M. M. Harpold, K. A. Stauderman, and D. Pietrobon. Functional consequences of mutations in the human alpha1 A calcium channel subunit linked to familial hemiplegic migraine. J. Neurosci., 19(5):1610-1619, Mar 1999.

L. P. Haynes, A. V. Tepikin, and R. D. Burgoyne. Calcium-binding protein 1 is an inhibitor of agonist-evoked, inositol 1,4,5-trisphosphate-mediated calcium signaling. J. Biol. Chem., 279 (1):547-555, Jan 2004.

L. P. Haynes, H. V. McCue, and R. D. Burgoyne. Evolution and functional diversity of the Calcium Binding Proteins (CaBPs). Front Mol Neurosci, 5:9, Jan 2012.

H.E. Heffner and R.S. Heffner. High-frequency hearing. The senses: a comprehensive reference, pages 55-60, 2008.

S. Helfmann, P. Neumann, K. Tittmann, T. Moser, R. Ficner, and E. Reisinger. The crystal structure of the $\mathrm{C} 2 \mathrm{~A}$ domain of otoferlin reveals an unconventional top loop region. J. Mol. Biol., 406(3):479-490, Feb 2011.

J. R. Holt, S. K. Gillespie, D. W. Provance, K. Shah, K. M. Shokat, D. P. Corey, J. A. Mercer, and P. G. Gillespie. A chemical-genetic strategy implicates myosin-1c in adaptation by hair cells. Cell, 108(3):371-381, Feb 2002.

M. B. Hoppa, B. Lana, W. Margas, A. C. Dolphin, and T. A. Ryan. alpha2delta expression sets presynaptic calcium channel abundance and release probability. Nature, 486(7401):122-125, Jun 2012.

H. Huang, D. Yu, and T. W. Soong. C-terminal alternative splicing of CaV1.3 channels distinctively modulates their dihydropyridine sensitivity. Mol. Pharmacol., 84(4):643-653, Oct 2013. 


\section{REFERENCES}

S. Imamura and J. C. Adams. Immunolocalization of peptide 19 and other calcium-binding proteins in the guinea pig cochlea. Anat. Embryol., 194(4):407-418, Oct 1996.

R. Iosub, D. Avitabile, L. Grant, K. Tsaneva-Atanasova, and H. J. Kennedy. Calcium-Induced calcium release during action potential firing in developing inner hair cells. Biophys. J., 108(5): 1003-1012, Mar 2015.

N. P. Issa and A. J. Hudspeth. The entry and clearance of Ca2+ at individual presynaptic active zones of hair cells from the bullfrog's sacculus. Proc. Natl. Acad. Sci. U.S.A., 93(18):95279532, Sep 1996.

S. L. Jackman, S. Y. Choi, W. B. Thoreson, K. Rabl, T. M. Bartoletti, and R. H. Kramer. Role of the synaptic ribbon in transmitting the cone light response. Nat. Neurosci., 12(3):303-310, Mar 2009.

M. A. Jenkins, C. J. Christel, Y. Jiao, S. Abiria, K. Y. Kim, Y. M. Usachev, G. J. Obermair, R. J. Colbran, and A. Lee. Ca2+-dependent facilitation of Cav1.3 Ca2+ channels by densin and Ca2+/calmodulin-dependent protein kinase II. J. Neurosci., 30(15):5125-5135, Apr 2010.

Z. Jing, M. A. Rutherford, H. Takago, T. Frank, A. Fejtova, D. Khimich, T. Moser, and N. Strenzke. Disruption of the presynaptic cytomatrix protein bassoon degrades ribbon anchorage, multiquantal release, and sound encoding at the hair cell afferent synapse. J. Neurosci., 33(10): 4456-4467, Mar 2013.

C. P. Johnson and E. R. Chapman. Otoferlin is a calcium sensor that directly regulates SNAREmediated membrane fusion. J. Cell Biol., 191(1):187-197, Oct 2010.

S. L. Johnson and W. Marcotti. Biophysical properties of CaV1.3 calcium channels in gerbil inner hair cells. J. Physiol. (Lond.), 586(4):1029-1042, Feb 2008.

S. L. Johnson, T. Eckrich, S. Kuhn, V. Zampini, C. Franz, K. M. Ranatunga, T. P. Roberts, S. Masetto, M. Knipper, C. J. Kros, and W. Marcotti. Position-dependent patterning of spontaneous action potentials in immature cochlear inner hair cells. Nat. Neurosci., 14(6):711-717, Jun 2011.

S. Jung, T. Maritzen, C. Wichmann, Z. Jing, A. Neef, N. H. Revelo, H. Al-Moyed, S. Meese, S. M. Wojcik, I. Panou, H. Bulut, P. Schu, R. Ficner, E. Reisinger, S. O. Rizzoli, J. Neef, N. Strenzke, V. Haucke, and T. Moser. Disruption of adaptor protein 2mu (AP-2mu) in cochlear hair cells impairs vesicle reloading of synaptic release sites and hearing. EMBO.J., In press, 2015a.

S. Jung, T. Oshima-Takago, R. Chakrabarti, A. B. Wong, Z. Jing, G. Yamanbaeva, M. M. Picher, S. M. Wojcik, F. Gottfert, F. Predoehl, K. Michel, S. W. Hell, S. Schoch, N. Strenzke, C. Wichmann, and T. Moser. Rab3-interacting molecules 2alpha and 2beta promote the abundance of 
voltage-gated CaV1.3 Ca2+ channels at hair cell active zones. Proc. Natl. Acad. Sci. U.S.A., 112(24):E3141-3149, Jun 2015b.

K. Kamiya, V. Michel, F. Giraudet, B. Riederer, I. Foucher, S. Papal, I. Perfettini, S. Le Gal, E. Verpy, W. Xia, U. Seidler, M. M. Georgescu, P. Avan, A. El-Amraoui, and C. Petit. An unusually powerful mode of low-frequency sound interference due to defective hair bundles of the auditory outer hair cells. Proc. Natl. Acad. Sci. U.S.A., 111(25):9307-9312, Jun 2014.

E. R. Kandel, J. H. Schwartz, and T. M. Jessell. Principles of Neural Science. McGraw-Hill, 4 edition, 2000. ISBN 0-8385-7701-6.

N. N. Kasri, A. M. Holmes, G. Bultynck, J. B. Parys, M. D. Bootman, K. Rietdorf, L. Missiaen, F. McDonald, H. De Smedt, S. J. Conway, A. B. Holmes, M. J. Berridge, and H. L. Roderick. Regulation of InsP3 receptor activity by neuronal Ca2+-binding proteins. EMBO J., 23(2): 312-321, Jan 2004.

Y. Kawashima, G. S. Geleoc, K. Kurima, V. Labay, A. Lelli, Y. Asai, T. Makishima, D. K. Wu, C. C. Della Santina, J. R. Holt, and A. J. Griffith. Mechanotransduction in mouse inner ear hair cells requires transmembrane channel-like genes. J. Clin. Invest., 121(12):4796-4809, Dec 2011.

P. Kazmierczak, H. Sakaguchi, J. Tokita, E. M. Wilson-Kubalek, R. A. Milligan, U. Muller, and B. Kachar. Cadherin 23 and protocadherin 15 interact to form tip-link filaments in sensory hair cells. Nature, 449(7158):87-91, Sep 2007.

H. J. Kennedy and R. W. Meech. Fast Ca2+ signals at mouse inner hair cell synapse: a role for Ca2+-induced Ca2+ release. J. Physiol. (Lond.), 539(Pt 1):15-23, Feb 2002.

T. Kharkovets, K. Dedek, H. Maier, M. Schweizer, D. Khimich, R. Nouvian, V. Vardanyan, R. Leuwer, T. Moser, and T. J. Jentsch. Mice with altered KCNQ4 K+ channels implicate sensory outer hair cells in human progressive deafness. EMBO J., 25(3):642-652, Feb 2006.

D. Khimich, R. Nouvian, R. Pujol, S. Tom Dieck, A. Egner, E. D. Gundelfinger, and T. Moser. Hair cell synaptic ribbons are essential for synchronous auditory signalling. Nature, 434(7035): 889-894, Apr 2005.

J. H. Kim, S. R. Lee, L. H. Li, H. J. Park, J. H. Park, K. Y. Lee, M. K. Kim, B. A. Shin, and S. Y. Choi. High cleavage efficiency of a 2 A peptide derived from porcine teschovirus-1 in human cell lines, zebrafish and mice. PLoS ONE, 6(4):e18556, 2011.

K. Y. Kim, E. S. Scholl, X. Liu, A. Shepherd, F. Haeseleer, and A. Lee. Localization and expression of CaBP1/caldendrin in the mouse brain. Neuroscience, 268:33-47, May 2014. 
M. Kinoshita-Kawada, J. Tang, R. Xiao, S. Kaneko, J. K. Foskett, and M. X. Zhu. Inhibition of TRPC5 channels by Ca2+-binding protein 1 in Xenopus oocytes. Pflugers Arch., 450(5): 345-354, Aug 2005.

S. I. Kitajiri, R. McNamara, T. Makishima, T. Husnain, A. U. Zafar, R. A. Kittles, Z. M. Ahmed, T. B. Friedman, S. Riazuddin, and A. J. Griffith. Identities, frequencies and origins of TMC1 mutations causing DFNB7/B11 deafness in Pakistan. Clin. Genet., 72(6):546-550, Dec 2007.

S. Kiyonaka, M. Wakamori, T. Miki, Y. Uriu, M. Nonaka, H. Bito, A. M. Beedle, E. Mori, Y. Hara, M. De Waard, M. Kanagawa, M. Itakura, M. Takahashi, K. P. Campbell, and Y. Mori. RIM1 confers sustained activity and neurotransmitter vesicle anchoring to presynaptic $\mathrm{Ca} 2+$ channels. Nat. Neurosci., 10(6):691-701, Jun 2007.

A. Koschak, D. Reimer, I. Huber, M. Grabner, H. Glossmann, J. Engel, and J. Striessnig. alpha 1D (Cav1.3) subunits can form 1-type Ca2+ channels activating at negative voltages. J. Biol. Chem., 276(25):22100-22106, Jun 2001.

R. L. Kraus, M. J. Sinnegger, H. Glossmann, S. Hering, and J. Striessnig. Familial hemiplegic migraine mutations change alpha1A Ca2+ channel kinetics. J. Biol. Chem., 273(10):55865590, Mar 1998.

R. L. Kraus, M. J. Sinnegger, A. Koschak, H. Glossmann, S. Stenirri, P. Carrera, and J. Striessnig. Three new familial hemiplegic migraine mutants affect P/Q-type $\mathrm{Ca}(2+)$ channel kinetics. $J$. Biol. Chem., 275(13):9239-9243, Mar 2000.

C. Kubisch, B. C. Schroeder, T. Friedrich, B. Lutjohann, A. El-Amraoui, S. Marlin, C. Petit, and T. J. Jentsch. KCNQ4, a novel potassium channel expressed in sensory outer hair cells, is mutated in dominant deafness. Cell, 96(3):437-446, Feb 1999.

S. Kuhn, M. Knirsch, L. Ruttiger, S. Kasperek, H. Winter, M. Freichel, V. Flockerzi, M. Knipper, and $\mathrm{J}$. Engel. Ba2+ currents in inner and outer hair cells of mice lacking the voltage-dependent Ca2+ channel subunits beta3 or beta4. Channels (Austin), 3(5):366-376, 2009.

A. Kuniyasu, K. Oka, T. Ide-Yamada, Y. Hatanaka, T. Abe, H. Nakayama, and Y. Kanaoka. Structural characterization of the dihydropyridine receptor-linked calcium channel from porcine heart. J. Biochem., 112(2):235-242, Aug 1992.

M. Lakso, J. G. Pichel, J. R. Gorman, B. Sauer, Y. Okamoto, E. Lee, F. W. Alt, and H. Westphal. Efficient in vivo manipulation of mouse genomic sequences at the zygote stage. Proc. Natl. Acad. Sci. U.S.A., 93(12):5860-5865, Jun 1996.

P. K. Legan, V. A. Lukashkina, R. J. Goodyear, M. Kossi, I. J. Russell, and G. P. Richardson. A targeted deletion in alpha-tectorin reveals that the tectorial membrane is required for the gain and timing of cochlear feedback. Neuron, 28(1):273-285, Oct 2000. 
C. Li, M. Enomoto, A. M. Rossi, M. D. Seo, T. Rahman, P. B. Stathopulos, C. W. Taylor, M. Ikura, and J. B. Ames. CaBP1, a neuronal $\mathrm{Ca} 2+$ sensor protein, inhibits inositol trisphosphate receptors by clamping intersubunit interactions. Proc. Natl. Acad. Sci. U.S.A., 110(21):8507-8512, May 2013.

L. D. Liberman, H. Wang, and M. C. Liberman. Opposing gradients of ribbon size and AMPA receptor expression underlie sensitivity differences among cochlear-nerve/hair-cell synapses. $J$. Neurosci., 31(3):801-808, Jan 2011.

M. C. Liberman. Auditory-nerve response from cats raised in a low-noise chamber. J. Acoust. Soc. Am., 63(2):442-455, Feb 1978.

M. C. Liberman. Single-neuron labeling in the cat auditory nerve. Science, 216(4551):1239-1241, Jun 1982.

M. C. Liberman and M. E. Oliver. Morphometry of intracellularly labeled neurons of the auditory nerve: correlations with functional properties. J. Comp. Neurol., 223(2):163-176, Feb 1984.

T. Maeda, J. Lem, K. Palczewski, and F. Haeseleer. A critical role of CaBP4 in the cone synapse. Invest. Ophthalmol. Vis. Sci., 46(11):4320-4327, Nov 2005.

V. G. Magupalli, K. Schwarz, K. Alpadi, S. Natarajan, G. M. Seigel, and F. Schmitz. Multiple RIBEYE-RIBEYE interactions create a dynamic scaffold for the formation of synaptic ribbons. J. Neurosci., 28(32):7954-7967, Aug 2008.

Z. F. Mann and M. W. Kelley. Development of tonotopy in the auditory periphery. Hear. Res., 276 (1-2):2-15, Jun 2011.

A. Matsubara, J. H. Laake, S. Davanger, S. Usami, and O. P. Ottersen. Organization of AMPA receptor subunits at a glutamate synapse: a quantitative immunogold analysis of hair cell synapses in the rat organ of Corti. J. Neurosci., 16(14):4457-4467, Jul 1996.

A. C. Meyer, T. Frank, D. Khimich, G. Hoch, D. Riedel, N. M. Chapochnikov, Y. M. Yarin, B. Harke, S. W. Hell, A. Egner, and T. Moser. Tuning of synapse number, structure and function in the cochlea. Nat. Neurosci., 12(4):444-453, Apr 2009.

D. L. Minor and F. Findeisen. Progress in the structural understanding of voltage-gated calcium channel (CaV) function and modulation. Channels (Austin), 4(6):459-474, 2010.

T. Moser. Gene therapy for deafness: How close are we? Sci Transl Med, 7(295):295fs28, Jul 2015.

T. Moser and D. Beutner. Kinetics of exocytosis and endocytosis at the cochlear inner hair cell afferent synapse of the mouse. Proc. Natl. Acad. Sci. U.S.A., 97(2):883-888, Jan 2000. 
T. Moser, F. Predoehl, and A. Starr. Review of hair cell synapse defects in sensorineural hearing impairment. Otol. Neurotol., 34(6):995-1004, Aug 2013.

J. Neef, A. Gehrt, A. V. Bulankina, A. C. Meyer, D. Riedel, R. G. Gregg, N. Strenzke, and T. Moser. The $\mathrm{Ca} 2+$ channel subunit beta2 regulates $\mathrm{Ca} 2+$ channel abundance and function in inner hair cells and is required for hearing. J. Neurosci., 29(34):10730-10740, Aug 2009.

A. Neely and P. Hidalgo. Structure-function of proteins interacting with the alpha1 pore-forming subunit of high-voltage-activated calcium channels. Front Physiol, 5:209, 2014.

R. A. Nicoll, S. Tomita, and D. S. Bredt. Auxiliary subunits assist AMPA-type glutamate receptors. Science, 311(5765):1253-1256, Mar 2006.

R. Nouvian, D. Beutner, T. D. Parsons, and T. Moser. Structure and function of the hair cell ribbon synapse. J. Membr. Biol., 209(2-3):153-165, 2006.

R. Nouvian, J. Neef, A. V. Bulankina, E. Reisinger, T. Pangršič, T. Frank, S. Sikorra, N. Brose, T. Binz, and T. Moser. Exocytosis at the hair cell ribbon synapse apparently operates without neuronal SNARE proteins. Nat. Neurosci., 14(4):411-413, Apr 2011.

D. Oliver, D. Z. He, N. Klocker, J. Ludwig, U. Schulte, S. Waldegger, J. P. Ruppersberg, P. Dallos, and B. Fakler. Intracellular anions as the voltage sensor of prestin, the outer hair cell motor protein. Science, 292(5525):2340-2343, Jun 2001.

S. Oz, V. Tsemakhovich, C. J. Christel, A. Lee, and N. Dascal. CaBP1 regulates voltage-dependent inactivation and activation of $\mathrm{Ca}(\mathrm{V}) 1.2$ (L-type) calcium channels. J. Biol. Chem., 286(16): 13945-13953, Apr 2011.

B. Pan, G. S. Geleoc, Y. Asai, G. C. Horwitz, K. Kurima, K. Ishikawa, Y. Kawashima, A. J. Griffith, and J. R. Holt. TMC1 and TMC2 are components of the mechanotransduction channel in hair cells of the mammalian inner ear. Neuron, 79(3):504-515, Aug 2013.

T. Pangršič, L. Lasarow, K. Reuter, H. Takago, M. Schwander, D. Riedel, T. Frank, L. M. Tarantino, J. S. Bailey, N. Strenzke, N. Brose, U. Muller, E. Reisinger, and T. Moser. Hearing requires otoferlin-dependent efficient replenishment of synaptic vesicles in hair cells. Nat. Neurosci., 13(7):869-876, Jul 2010.

T. Pangršič, E. Reisinger, and T. Moser. Otoferlin: a multi-C2 domain protein essential for hearing. Trends Neurosci., 35(11):671-680, Nov 2012.

T. Pangršič, M. Gabrielaitis, S. Michanski, B. Schwaller, F. Wolf, N. Strenzke, and T. Moser. EFhand protein $\mathrm{Ca} 2+$ buffers regulate $\mathrm{Ca} 2+$ influx and exocytosis in sensory hair cells. Proc. Natl. Acad. Sci. U.S.A., 112(9):E1028-1037, Mar 2015. 
T. D. Parsons and P. Sterling. Synaptic ribbon. Conveyor belt or safety belt? Neuron, 37(3): 379-382, Feb 2003.

A. Pinggera, A. Lieb, B. Benedetti, M. Lampert, S. Monteleone, K. R. Liedl, P. Tuluc, and J. Striessnig. CACNA1D de novo mutations in autism spectrum disorders activate Cav1.3 Ltype calcium channels. Biol. Psychiatry, 77(9):816-822, May 2015.

G. S. Pitt. Calmodulin and CaMKII as molecular switches for cardiac ion channels. Cardiovasc. Res., 73(4):641-647, Mar 2007.

J. Platzer, J. Engel, A. Schrott-Fischer, K. Stephan, S. Bova, H. Chen, H. Zheng, and J. Striessnig. Congenital deafness and sinoatrial node dysfunction in mice lacking class D L-type Ca2+ channels. Cell, 102(1):89-97, Jul 2000.

D. Purves, G.J. Augustine, and D. et al. Fitzpatrick. Neuroscience, volume 3. Sinauer Associates, Sunderland (MA), 2 edition, 2001.

N. A. Ramakrishnan, M. J. Drescher, and D. G. Drescher. Direct interaction of otoferlin with syntaxin 1A, SNAP-25, and the L-type voltage-gated calcium channel Cav1.3. J. Biol. Chem., 284(3):1364-1372, Jan 2009.

H. Regus-Leidig, C. Ott, M. Lohner, J. Atorf, M. Fuchs, T. Sedmak, J. Kremers, A. Fejtova, E. D. Gundelfinger, and J. H. Brandstatter. Identification and immunocytochemical characterization of Piccolino, a novel Piccolo splice variant selectively expressed at sensory ribbon synapses of the eye and ear. PLOS ONE, 8(8):e70373, 2013.

E. Reisinger, C. Bresee, J. Neef, R. Nair, K. Reuter, A. Bulankina, R. Nouvian, M. Koch, J. Buckers, L. Kastrup, I. Roux, C. Petit, S. W. Hell, N. Brose, J. S. Rhee, S. Kugler, J. V. Brigande, and T. Moser. Probing the functional equivalence of otoferlin and synaptotagmin 1 in exocytosis. $J$. Neurosci., 31(13):4886-4895, Mar 2011.

S. Restituito, T. Cens, C. Barrere, S. Geib, S. Galas, M. De Waard, and P. Charnet. The [beta]2a subunit is a molecular groom for the Ca2+ channel inactivation gate. J. Neurosci., 20(24): 9046-9052, Dec 2000.

K. Reuter. Biochemistry and physiological role of otoferlin . Göttingen, Aug 2012.

A. J. Ricci, Y. C. Wu, and R. Fettiplace. The endogenous calcium buffer and the time course of transducer adaptation in auditory hair cells. J. Neurosci., 18(20):8261-8277, Oct 1998.

G. P. Richardson, A. N. Lukashkin, and I. J. Russell. The tectorial membrane: one slice of a complex cochlear sandwich. Curr Opin Otolaryngol Head Neck Surg, 16(5):458-464, Oct 2008. 
F. Rieke, A. Lee, and F. Haeseleer. Characterization of Ca2+-binding protein 5 knockout mouse retina. Invest. Ophthalmol. Vis. Sci., 49(11):5126-5135, Nov 2008.

W. M. Roberts. Spatial calcium buffering in saccular hair cells. Nature, 363(6424):74-76, May 1993.

D. Robertson and B. Paki. Role of L-type Ca2+ channels in transmitter release from mammalian inner hair cells. II. Single-neuron activity. J. Neurophysiol., 87(6):2734-2740, Jun 2002.

M. Rodriguez-Ballesteros, F. J. del Castillo, Y. Martin, M. A. Moreno-Pelayo, C. Morera, F. Prieto, J. Marco, A. Morant, J. Gallo-Teran, C. Morales-Angulo, C. Navas, G. Trinidad, M. C. Tapia, F. Moreno, and I. del Castillo. Auditory neuropathy in patients carrying mutations in the otoferlin gene (OTOF). Hum. Mutat., 22(6):451-456, Dec 2003.

I. Roux, S. Safieddine, R. Nouvian, M. Grati, M. C. Simmler, A. Bahloul, I. Perfettini, M. Le Gall, P. Rostaing, G. Hamard, A. Triller, P. Avan, T. Moser, and C. Petit. Otoferlin, defective in a human deafness form, is essential for exocytosis at the auditory ribbon synapse. Cell, 127(2): 277-289, Oct 2006.

J. Ruel, S. Emery, R. Nouvian, T. Bersot, B. Amilhon, J. M. Van Rybroek, G. Rebillard, M. Lenoir, M. Eybalin, B. Delprat, T. A. Sivakumaran, B. Giros, S. El Mestikawy, T. Moser, R. J. Smith, M. M. Lesperance, and J. L. Puel. Impairment of SLC17A8 encoding vesicular glutamate transporter-3, VGLUT3, underlies nonsyndromic deafness DFNA25 and inner hair cell dysfunction in null mice. Am. J. Hum. Genet., 83(2):278-292, Aug 2008.

I. J. Russell and P. M. Sellick. Tuning properties of cochlear hair cells. Nature, 267(5614):858860, Jun 1977.

S. Safieddine and R. J. Wenthold. SNARE complex at the ribbon synapses of cochlear hair cells: analysis of synaptic vesicle- and synaptic membrane-associated proteins. Eur. J. Neurosci., 11 (3):803-812, Mar 1999.

S. Safieddine, A. El-Amraoui, and C. Petit. The auditory hair cell ribbon synapse: from assembly to function. Annu. Rev. Neurosci., 35:509-528, 2012.

H. Sakaguchi, J. Tokita, U. Muller, and B. Kachar. Tip links in hair cells: molecular composition and role in hearing loss. Curr Opin Otolaryngol Head Neck Surg, 17(5):388-393, Oct 2009.

Anja Scharinger, Stephanie Eckrich, David H Vandael, Kai Schönig, Alexandra Koschak, Dietmar Hecker, Gurjot Kaur, Amy Lee, Anupam Sah, Dusan Bartsch, Bruno Benedetti, Andreas Lieb, Bernhard Schick, Nicolas Singewald, Martina J Brauns, Emilio Carbone, Jutta Engel, and Joerg Striessnig. Cell-type-specific tuning of cav1.3 ca2+-channels by a c-terminal automodulatory domain. Frontiers in Cellular Neuroscience, 9(309), 2015. ISSN 1662-5102. doi: 10.3389/ fncel.2015.00309. 
F. Schmitz, A. Konigstorfer, and T. C. Sudhof. RIBEYE, a component of synaptic ribbons: a protein's journey through evolution provides insight into synaptic ribbon function. Neuron, 28 (3):857-872, Dec 2000.

M. E. Schnee, J. Santos-Sacchi, M. Castellano-Munoz, J. H. Kong, and A. J. Ricci. Calciumdependent synaptic vesicle trafficking underlies indefatigable release at the hair cell afferent fiber synapse. Neuron, 70(2):326-338, Apr 2011.

C. A. Schneider, W. S. Rasband, and K. W. Eliceiri. NIH Image to ImageJ: 25 years of image analysis. Nat. Methods, 9(7):671-675, Jul 2012.

I. Schrauwen, S. Helfmann, A. Inagaki, F. Predoehl, M. A. Tabatabaiefar, M. M. Picher, M. Sommen, C. Z. Seco, J. Oostrik, H. Kremer, A. Dheedene, C. Claes, E. Fransen, M. H. Chaleshtori, P. Coucke, A. Lee, T. Moser, and G. Van Camp. A mutation in CABP2, expressed in cochlear hair cells, causes autosomal-recessive hearing impairment. Am. J. Hum. Genet., 91(4):636-645, Oct 2012.

R. P. Seal, O. Akil, E. Yi, C. M. Weber, L. Grant, J. Yoo, A. Clause, K. Kandler, J. L. Noebels, E. Glowatzki, L. R. Lustig, and R. H. Edwards. Sensorineural deafness and seizures in mice lacking vesicular glutamate transporter 3. Neuron, 57(2):263-275, Jan 2008.

Y. Shen, D. Yu, H. Hiel, P. Liao, D. T. Yue, P. A. Fuchs, and T. W. Soong. Alternative splicing of the $\mathrm{Ca}(\mathrm{v}) 1.3$ channel IQ domain, a molecular switch for $\mathrm{Ca} 2+$-dependent inactivation within auditory hair cells. J. Neurosci., 26(42):10690-10699, Oct 2006.

J. Siemens, C. Lillo, R. A. Dumont, A. Reynolds, D. S. Williams, P. G. Gillespie, and U. Muller. Cadherin 23 is a component of the tip link in hair-cell stereocilia. Nature, 428(6986):950-955, Apr 2004.

A. Singh, M. Gebhart, R. Fritsch, M. J. Sinnegger-Brauns, C. Poggiani, J. C. Hoda, J. Engel, C. Romanin, J. Striessnig, and A. Koschak. Modulation of voltage- and Ca2+-dependent gating of CaV1.3 L-type calcium channels by alternative splicing of a C-terminal regulatory domain. J. Biol. Chem., 283(30):20733-20744, Jul 2008.

A. Sirmaci, D. Duman, H. Ozturkmen-Akay, S. Erbek, A. Incesulu, B. Ozturk-Himi, Z. S. Arici, E. B. Yuksel-Konuk, S. Tair-Yilmaz, S. Tokgoz-Yilmaz, F. B. Cengiz, I. Aslan, M. Yildirim, A. Hasanefendiolu-Bayrak, A. Aycicek, I. Yilmaz, S. Fitoz, F. Altin, H. Ozda, and M. Tekin. Mutations in TMC1 contribute significantly to nonsyndromic autosomal recessive sensorineural hearing loss: a report of five novel mutations. Int. J. Pediatr. Otorhinolaryngol., 73(5):699-705, May 2009.

W. C. Skarnes, B. Rosen, A. P. West, M. Koutsourakis, W. Bushell, V. Iyer, A. O. Mujica, M. Thomas, J. Harrow, T. Cox, D. Jackson, J. Severin, P. Biggs, J. Fu, M. Nefedov, P. J. de Jong, 
A. F. Stewart, and A. Bradley. A conditional knockout resource for the genome-wide study of mouse gene function. Nature, 474(7351):337-342, Jun 2011.

R.J.H. Smith, A.E. Shearer, and M.S. et al. Hildebrand. Deafness and Hereditary Hearing Loss Overview, volume last updated Jan 9, 2014. GeneReviewsÂA, Seattle (WA): University of Washington, Feb 1999. updated 2014 Jan 9.

I. Sokal, N. Li, C. L. Verlinde, F. Haeseleer, W. Baehr, and K. Palczewski. Ca(2+)-binding proteins in the retina: from discovery to etiology of human disease(1). Biochim. Biophys. Acta, 1498 (2-3):233-251, Dec 2000.

I. Splawski, K. W. Timothy, L. M. Sharpe, N. Decher, P. Kumar, R. Bloise, C. Napolitano, P. J. Schwartz, R. M. Joseph, K. Condouris, H. Tager-Flusberg, S. G. Priori, M. C. Sanguinetti, and M. T. Keating. $\mathrm{Ca}(\mathrm{V}) 1.2$ calcium channel dysfunction causes a multisystem disorder including arrhythmia and autism. Cell, 119(1):19-31, Oct 2004.

I. Splawski, K. W. Timothy, N. Decher, P. Kumar, F. B. Sachse, A. H. Beggs, M. C. Sanguinetti, and M. T. Keating. Severe arrhythmia disorder caused by cardiac L-type calcium channel mutations. Proc. Natl. Acad. Sci. U.S.A., 102(23):8089-8096, Jun 2005.

P. Sterling and G. Matthews. Structure and function of ribbon synapses. Trends Neurosci., 28(1): 20-29, Jan 2005.

S. C. Stotz and G. W. Zamponi. Identification of inactivation determinants in the domain IIS6 region of high voltage-activated calcium channels. J. Biol. Chem., 276(35):33001-33010, Aug 2001.

S. C. Stotz, J. Hamid, R. L. Spaetgens, S. E. Jarvis, and G. W. Zamponi. Fast inactivation of voltage-dependent calcium channels. A hinged-lid mechanism? J. Biol. Chem., 275(32):2457524582, Aug 2000.

M. A. Tabatabaiefar, F. Alasti, L. Shariati, E. Farrokhi, E. Fransen, M. R. Nooridaloii, M. H. Chaleshtori, and G. Van Camp. DFNB93, a novel locus for autosomal recessive moderate-tosevere hearing impairment. Clin. Genet., 79(6):594-598, Jun 2011.

A. M. Taberner and M. C. Liberman. Response properties of single auditory nerve fibers in the mouse. J. Neurophysiol., 93(1):557-569, Jan 2005.

M. R. Tadross, M. Ben Johny, and D. T. Yue. Molecular endpoints of Ca2+/calmodulin- and voltage-dependent inactivation of Ca(v)1.3 channels. J. Gen. Physiol., 135(3):197-215, Mar 2010. 
B. Z. Tan, F. Jiang, M. Y. Tan, D. Yu, H. Huang, Y. Shen, and T. W. Soong. Functional characterization of alternative splicing in the $\mathrm{C}$ terminus of L-type CaV1.3 channels. J. Biol. Chem., 286 (49):42725-42735, Dec 2011.

N. Tang, T. Lin, J. Yang, J. K. Foskett, and E. M. Ostap. CIB1 and CaBP1 bind to the myo1c regulatory domain. J. Muscle Res. Cell. Motil., 28(6):285-291, 2007.

S. tom Dieck, D. Specht, N. Strenzke, Y. Hida, V. Krishnamoorthy, K. F. Schmidt, E. Inoue, H. Ishizaki, M. Tanaka-Okamoto, J. Miyoshi, A. Hagiwara, J. H. Brandstatter, S. Lowel, T. Gollisch, T. Ohtsuka, and T. Moser. Deletion of the presynaptic scaffold CAST reduces active zone size in rod photoreceptors and impairs visual processing. J. Neurosci., 32(35):12192-12203, Aug 2012.

T. Toth, L. Deak, F. Fazakas, J. Zheng, L. Muszbek, and I. Sziklai. A new mutation in the human pres gene and its effect on prestin function. Int. J. Mol. Med., 20(4):545-550, Oct 2007.

V. Tsuprun and P. Santi. Ultrastructure and immunohistochemical identification of the extracellular matrix of the chinchilla cochlea. Hear. Res., 129(1-2):35-49, Mar 1999.

F. Van Petegem, K. E. Duderstadt, K. A. Clark, M. Wang, and D. L. Minor. Alanine-scanning mutagenesis defines a conserved energetic hotspot in the CaValpha1 AID-CaVbeta interaction site that is critical for channel modulation. Structure, 16(2):280-294, Feb 2008.

E. Verpy, M. Leibovici, N. Michalski, R. J. Goodyear, C. Houdon, D. Weil, G. P. Richardson, and C. Petit. Stereocilin connects outer hair cell stereocilia to one another and to the tectorial membrane. J. Comp. Neurol., 519(2):194-210, Feb 2011.

C. Vogl, B. H. Cooper, J. Neef, S. M. Wojcik, K. Reim, E. Reisinger, N. Brose, J. S. Rhee, T. Moser, and C. Wichmann. Unconventional molecular regulation of synaptic vesicle replenishment in cochlear inner hair cells. J. Cell. Sci., 128(4):638-644, Feb 2015.

C. Wichmann and T. Moser. Relating structure and function of inner hair cell ribbon synapses. Cell Tissue Res., 361(1):95-114, Jul 2015.

J. N. Wingard, J. Chan, I. Bosanac, F. Haeseleer, K. Palczewski, M. Ikura, and J. B. Ames. Structural analysis of $\mathrm{Mg} 2+$ and $\mathrm{Ca} 2+$ binding to $\mathrm{CaBP} 1$, a neuron-specific regulator of calcium channels. J. Biol. Chem., 280(45):37461-37470, Nov 2005.

A. B. Wong, M. A. Rutherford, M. Gabrielaitis, T. Pangršič, F. Gottfert, T. Frank, S. Michanski, S. Hell, F. Wolf, C. Wichmann, and T. Moser. Developmental refinement of hair cell synapses tightens the coupling of Ca2+ influx to exocytosis. EMBO J., 33(3):247-264, Feb 2014. 
P. S. Yang, B. A. Alseikhan, H. Hiel, L. Grant, M. X. Mori, W. Yang, P. A. Fuchs, and D. T. Yue. Switching of $\mathrm{Ca} 2+-$ dependent inactivation of $\mathrm{Ca}(\mathrm{v}) 1.3$ channels by calcium binding proteins of auditory hair cells. J. Neurosci., 26(42):10677-10689, Oct 2006.

P. S. Yang, M. B. Johny, and D. T. Yue. Allostery in Ca2+ channel modulation by calcium-binding proteins. Nat. Chem. Biol., 10(3):231-238, Mar 2014.

S. Yasunaga, M. Grati, M. Cohen-Salmon, A. El-Amraoui, M. Mustapha, N. Salem, E. El-Zir, J. Loiselet, and C. Petit. A mutation in OTOF, encoding otoferlin, a FER-1-like protein, causes DFNB9, a nonsyndromic form of deafness. Nat. Genet., 21(4):363-369, Apr 1999.

V. Zampini, S. L. Johnson, C. Franz, N. D. Lawrence, S. Munkner, J. Engel, M. Knipper, J. Magistretti, S. Masetto, and W. Marcotti. Elementary properties of $\mathrm{CaV} 1.3 \mathrm{Ca}(2+)$ channels expressed in mouse cochlear inner hair cells. J. Physiol. (Lond.), 588(Pt 1):187-199, Jan 2010.

C. Zeitz, B. Kloeckener-Gruissem, U. Forster, S. Kohl, I. Magyar, B. Wissinger, G. Matyas, F. X. Borruat, D. F. Schorderet, E. Zrenner, F. L. Munier, and W. Berger. Mutations in CABP4, the gene encoding the $\mathrm{Ca} 2+$-binding protein 4 , cause autosomal recessive night blindness. Am. J. Hum. Genet., 79(4):657-667, Oct 2006.

H. Zhang, A. Maximov, Y. Fu, F. Xu, T. S. Tang, T. Tkatch, D. J. Surmeier, and I. Bezprozvanny. Association of CaV1.3 L-type calcium channels with Shank. J. Neurosci., 25(5):1037-1049, Feb 2005.

J. Zheng, W. Shen, D. Z. He, K. B. Long, L. D. Madison, and P. Dallos. Prestin is the motor protein of cochlear outer hair cells. Nature, 405(6783):149-155, May 2000.

H. Zhou, S. A. Kim, E. A. Kirk, A. L. Tippens, H. Sun, F. Haeseleer, and A. Lee. Ca2+-binding protein-1 facilitates and forms a postsynaptic complex with Cav1.2 (L-type) Ca2+ channels. $J$. Neurosci., 24(19):4698-4708, May 2004.

H. Zhou, K. Yu, K. L. McCoy, and A. Lee. Molecular mechanism for divergent regulation of Cav1.2 Ca2+ channels by calmodulin and Ca2+-binding protein-1. J. Biol. Chem., 280(33): 29612-29619, Aug 2005.

A. Zuccotti, S. Clementi, T. Reinbothe, A. Torrente, D. H. Vandael, and A. Pirone. Structural and functional differences between L-type calcium channels: crucial issues for future selective targeting. Trends Pharmacol. Sci., 32(6):366-375, Jun 2011. 


\section{Acknowledgements}

I would like to express my gratitude to my supervisor Tobias Moser for the opportunity to work in his lab and for teaching me patch-clamp. I am grateful for his guidance and constant support during my $\mathrm{PhD}$, his fast solution-oriented thinking and encouragement to scientific representations. I want to thank my thesis committee members Fred Wolf and Tomas Dresbach for scientific suggestions, supervising the progress of the project.

I appreciate the collaboration with Amy Lee and Akhira Inagaki who taught me patch-clamp recordings of HEK cells and together with Tuck Wah Soong provided me with DNA plasmids. In this respect I would like to thank Luis Pardo for generating the HEK 293/hSK3-1 cell line and Aleksandra Ivanovic, Friederike Predoehl, Isabelle Schrauwen for organizing and generating the $\mathrm{Cabp} 2^{\mathrm{LacZ} / \mathrm{LacZ}}$ strain used during this research project.

I am in debt to Cristian Vogel for the never ending professional conversations, his troubleshooting and proof reading skills, all the encouraging conversations and his selfless support whenever needed. I am grateful for the help of of Tina Pangršič, her in depth analytical thinking, long and detailed scientific discussions and her critical reflections of my data. I want to thank Jakob Neef who always has an answer to an addressed question, taught me programming in Igor and is never too busy to provide a helping hand.

I want to thank Christiane Senger-Freitag and Sandra Gerke, for assisting during cloning procedures, their professional genotyping skills and maintaining an organized structured environment in the lab. I appreciate the help of Sangyong Jung in performing the otocyst injections, his motivating and optimistic working style and the support of Alexandra Müller during molecular biological assays.

Many thanks go to Stefan Thom, Nadine Herrmann, Gerhard Hoch and Mark Schmidt for expert technical assistance during this project. In that respect I also want to express my gratitude to Anna Gehrt and Nicola Strenzke for performing in vivo recordings and providing me with essential information about my project.

I am grateful for all the fun scientific and private discussions with Tanvi Butola and Chao-Hua Huang establishing an enjoyable working-atmosphere during long experimental procedures. I appreciate the creativity of Carlos Duque Afonso and his professional image processing skills. I am very thankful to all the members of the former InnerEarLab and the Institute for Auditory Neuroscience for establishing a positive, supportive and cheerful environment during this past years.

Moreover, I am thankful for the financial support by the German Research Foundation through the priority program 1608, the Bernstein Center for Computational Neuroscience and the Flemish 
National Fund for Scientific Research.

Finally, a tremendous thanks to my family who rendered the course of my $\mathrm{PhD}$, for their support, encouragement and understanding in all possible aspects; and most importantly I want to thank Marcus Huber for his endorsement at any time, his flexibility in changing workplace, for backing me when needed and all the bright conversations during late night skype calls. 


\section{Appendix}

Table 8.1: Cloning/RT-PCR primer sequences used throughout this study

\begin{tabular}{ll}
\hline Endconstruct & Primer $5^{6} \rightarrow 3^{6}:$ \\
\hline \hline $\begin{array}{l}\text { pEGFP-N1-wt-CABP2 } \\
\text { OR -EF1 }{ }^{*}\end{array}$ & $\begin{array}{l}\text { NheI fwd: GAGAGCTAGCGATGGGGAACTGTGCCAAGCGG } \\
\text { BamHI rev: TCTCTGGATCCCGAGACATCATTCGCAC }\end{array}$ \\
pEGFP-N1-CABP2- & NheI fwd: GAGAGCTAGCGATGGGGAACTGTGCCAAGCGG \\
EF & BamHI rev2: TCTCTGGATCCCGCTCCCGGAAGGCGTCCCG \\
& \\
AAV2-HBA-EWB- & Fwd AAV2: GAGAGAATTCATGGTTCAGAGACC \\
mCaBP2 & Rev AAV2: GAGAATTCGCCACCATGGTTCAG \\
& \\
RT PCR of mCabp2 & $\begin{array}{l}\text { exon 1 fwd: AGAGACCCATGGGGAACTG } \\
\text { exon 5 rev: CCCATCAGCTCCACAAAATC }\end{array}$ \\
& LacZ fwd: CCGGATTGATGGTAGTGGTC \\
& GAPDH fwd: ACTCCACTCACGGCAAATTC \\
& GAPDH rev: CACATTGGGGGTAGGAACAC \\
\hline
\end{tabular}

Table 8.2: Sequencing primers used throughout this study

\begin{tabular}{lll}
\hline Target & Name & Primer $5^{6} \rightarrow 3^{\boldsymbol{6}}:$ \\
\hline \hline & & \\
Long Range PCR: & & \\
\hline 5' target & 25502 & Rev: CACAACGGGTTCTTCTGTTAGTCC \\
5' target & 25503 & Fwd: CTGTCCATGCTGTTGGTATCCATAATTCCG \\
5' target & 25504 & Fwd: GTCCATGCTGTTGGTATCCATAATTCCGAG \\
3' target & 25505 & Rev: GTAGACTTAGTCGTAGTACCATGATG \\
3' target & 25506 & Rev: CATGACTTCAGGTGGCAGACTGGCTACTC \\
3' target & 25507 & Fwd: TCTATAGTCGCAGTAGGCGG \\
\hline \hline
\end{tabular}




\section{Abbreviations}

\begin{tabular}{|c|c|}
\hline AAV & adeno-associated virus \\
\hline ABR & auditory brainstem responses \\
\hline AID & alpha interaction domain \\
\hline AMPA receptors & $\alpha$-amino-3-hydroxy-5-methyl-4-isoxazole propionic acid receptors \\
\hline AP & action potential \\
\hline ATP & adenosine triphosphate \\
\hline $\mathrm{AZ}$ & active zone \\
\hline BAPTA & 1,2-bis(2-aminophenoxy)ethane-N,N,N',N'-tetraacetatic acid \\
\hline CABP & human $\mathrm{Ca}^{2+}$ binding protein \\
\hline Cabp & mouse $\mathrm{Ca}^{2+}$ binding protein \\
\hline $\mathrm{CaBP}$ & $\mathrm{Ca}^{2+}$ binding protein in general \\
\hline $\mathrm{CaM}$ & calmodulin \\
\hline CAPS & $\mathrm{Ca}^{2+}$-dependent activator proteins for secretion \\
\hline $\mathrm{CDH} 23$ & cadherin 23 \\
\hline CDI & $\mathrm{Ca}^{2+}$ dependent inactivation \\
\hline cDNA & complementary deoxyribonucleic acid \\
\hline $\mathrm{CF}$ & characteristic frequency \\
\hline CICR & $\mathrm{Ca}^{2+}$ induced $\mathrm{Ca}^{2+}$ release \\
\hline $\mathrm{C}_{m}$ & membrane capacitance \\
\hline$\Delta C_{m}$ & change in membrane capacitance \\
\hline $\mathrm{CN}$ & cochlear nucleus \\
\hline CSNB2 & congenital stationary night blindness 2 \\
\hline DCRD & distal C-terminal regulatory domain \\
\hline DFNA & deafness, autosomal dominant \\
\hline DFNB & deafness, autosomal recessive \\
\hline DKO & double knock-out \\
\hline dNTP & deoxynucleotide triphosphate \\
\hline DPOAE & distortion product otoacoustic emissions \\
\hline EDTA & ethylenediaminetetraacetic acid \\
\hline EGFP & enhanced green fluorescent protein \\
\hline EGTA & ethylene glycol-bis(2-aminoethylether)-N,N,N',N-tetraacetic acid \\
\hline ERG & electroretinograms \\
\hline $\mathrm{EtOH}$ & ethanol \\
\hline GAPDH & glyceraldehyde-3-phosphate dehydrogenase \\
\hline GPI-anchor & glycosylphosphatidylinositol \\
\hline GK & guanylate kinase \\
\hline GSDB & goat serum dilution buffer \\
\hline HBA promotor & human beta actin promotor \\
\hline HEK cell line & human embryonic kidney cell line \\
\hline HEPES & 4-(2-hydroxyethyl)-1-piperazineethanesulfonic acid \\
\hline hSK3 & human small-conductance $\mathrm{Ca}^{2+}$ activated $\mathrm{K}^{+}$channel \\
\hline $\mathrm{I}_{B a}$ & $\mathrm{Ba}^{2+}$ current \\
\hline $\mathrm{I}_{C a}$ & $\mathrm{Ca}^{2+}$ current \\
\hline
\end{tabular}


IHC

IP3 receptor

IQ

IRES

$\mathrm{k}_{\text {act }}$

$\mathrm{K}_{d}$

KOMP

LTCC

LR-PCR

MET

MIDAS

Munc13

mRNA

NMDG

n.s.

$\mathrm{OHC}$

PBS

PCR

PCH15

PCRD

PFA

$\mathrm{Q}_{C a}$

$\mathrm{R}_{500}$

RIM

RRP

$\mathrm{RS}$

RT-PCR

PSTH

SANDD

SDS

s.e.m.

SGN

SH3 domain

SK3 channel

SRP

TEA

Tris

Tmc1

VDI

VGCC

Vglut3

$\mathrm{V}_{\text {half }}$

$\mathrm{V}_{\text {holding }}$

WPRE

wt inner hair cell

inositol 1,4,5 triphosphate receptors

isoleucin-glutamin motive

internal ribosomes entry site

activation slope factor

dissociation constant

Knockout Mouse Project

L-type $\mathrm{Ca}^{2+}$ channel

long range polymerase chain reaction

mechanoelectrical transduction

metal ion-dependent adhesion site

mammalian uncoordinated 13

messenger ribonucleic acid

N-methyl-D-glucamine

not statistically significant

outer hair cell

phosphate buffered saline

polymerase chain reaction

protocadherin 15

proximal C-terminal regulatory domain

paraformaldehyde

charge integral of $\mathrm{Ca}^{2+}$ current

residual mean current after $500 \mathrm{~ms}$

Rab-3 interacting molecules

readily releasable pool

series resistance

reverse transcription polymerase chain reaction

peristimulus time histogram

sinoatrial node dysfunction and deafness

sodium dodecyl sulfate

standard error of the mean

spiral ganglion neuron

SRC homology 3 domain

small conductance $\mathrm{Ca}^{2+}$-activated potassium channel 3

sustained releasable pool

tetraethylammonium

Tris(hydroxymethyl)aminomethane

transmembrane channel-like 1

voltage-dependent inactivation

voltage-gated $\mathrm{Ca}^{2+}$ channel

vesicular glutamate transporter-3

voltage of half maximal current

holding potential

woodchuck post-transcriptional regulatory element

wild-type 


\section{Publication List}

Schrauwen I, Helfmann S, Inagaki A, Predoehl F, Tabatabaiefar MA, Picher MM, Sommen M, Seco CZ, Oostrik J, Kremer H, Dheedene A, Claes C, Fransen E, Chaleshtori MH, Coucke P, Lee A, Moser T, Van Camp F. "A mutation in $\mathrm{Ca}^{2+}$ binding protein 2, expressed in cochlear hair cells, causes autosomal recessive hearing impairment", Am. J. Hum. Genet. 91, 636-645 (2012)

Jung S, Oshima-Takago T, Chakrabarti R, Wong AB, Jing Z, Yamanbaeva G, Picher MM, Wojcik SM, Göttfert F, Predoehl F, Michel K, Hell SW, Schoch S, Strenzke N, Wichmann C, Moser T. "Rab3-interacting molecules $2 \alpha$ and $2 \beta$ promote the abundance of voltage-gated $\mathrm{Ca}_{V} 1.3 \mathrm{Ca}^{2+}$ channels at hair cell active zones", Proc. Natl. Acad. Sci. U. S. A. 112, E3141-3149 (2015)

Picher MM, Gehrt A, Meese S, Ivanovic A, Predoehl F, Schrauwen I, Jung S, Strenzke N and Moser T. " $\mathrm{Ca}^{2+}$-binding protein 2, defective in human hearing impairment, inhibits $\mathrm{Ca}^{2+}$ channel inactivation and contributes to $\mathrm{Ca}^{2+}$-buffering in sensory hair cells", (under review), (2015) 


\title{
Curriculum Vitae
}

\author{
Dipl.Ing. Maria Magdalena Picher (MSc)
}

\section{Personal Data}

$\begin{array}{ll}\text { Name: } & \begin{array}{l}\text { Maria Magdalena Picher } \\ \text { Weender Landstraße } 86\end{array} \\ & \begin{array}{l}\text { 37075 Göttingen } \\ \text { mm.picher@gmail.com }\end{array} \\ \text { E-mail Address: } & \begin{array}{l}\text { 07. June 1984/ Vienna, Austria } \\ \text { Date/Place of birth: }\end{array} \\ \text { Nationality: } & \text { Austria }\end{array}$

Education

2011-present

PhD in Göttingen Graduate School for Neurosciences, Biophysics, and Molecular Biosciences, focused on Sensory and Motorneuroscience/ University of Göttingen/ Göttingen/ Germany

PhD-Thesis in the Institute for Auditory Neuroscience: "The role of Calcium Binding Protein 2 in synaptic sound encoding and hearing"

Molecular biology, focus on Neuroscience; University of Vienna

$2007-2010$
MSc degree in Biotechnology at the University of Natural Resources and Applied Life Science/ Vienna/ Austria
Master-Thesis in the Department of Nanobiotechnology of the University of Natural Resources and Applied Life Science AND the Department of Nanosystems of the Austrian Institute of Technology: " Nanobiotechnology advanced Lab-on-a-Chip for continuous blood glucose monitoring" Awarded: $30^{\text {th }}$ of September, 2010

\section{Conferences / Symposia}

\section{Poster Presentations:}

$11^{\text {th }}$ Goettingen Meeting of the German Neuroscience Society: MM. Picher, A. Gehrt, S. Jung, I. Schrauwen, G. Van Camp and T. Moser: “The Role of Calcium Binding Protein 2 in Synaptic Sound Encoding and hearing" Göttingen/ Germany/ Mar. 2015

DFG Thematic conference on ultrafast and temporally precise information processing: MM. Picher, S. Jung, I. Schrauwen and T. Moser: “The Role of Calcium Binding Protein 2 in Synaptic Sound Encoding and hearing” Bad Münster/ Germany/ Sep. 2014

Association for Research in Otolaryngology-37th Annual MidWinter Meeting: MM. Picher, A. Ivanovic, F. Predoehl, G. Van Camp and T. Moser: "The Role of Calcium Binding Protein 2 in Synaptic Sound Encoding and hearing" San Diego/ USA/ Feb. 2014

$1^{\text {th }}$ Goettingen Meeting of the German Neuroscience Society: MM. Picher, I. Schrauwen, S. Helfmann, A. Inagaki, A. Lee, G. Van Camp, F. Wolf and T. Moser: "Biophysical properties of Inner Hair Cell Cav1.3 Ca ${ }^{2+}$ channels“ Göttingen/ Germany/ Mar.2013

Workshops/Current trends in Biomedicine 2012: Molecular mechanisms of inner ear development MM.Picher, TL.Wang and T. Moser „Mechanisms underlying heterogeneity of $\mathrm{Ca}^{2+}$ signaling among hair cell active zones" Baeza/ Spain/ Nov. 2012 
Bernstein Center of Computational Neuroscience visit 2012:MM. Picher, I. Schrauwen, S. Helfmann, A. Inagaki, A. Lee, G. Van Camp, F. Wolf and T. Moser: “Biophysical properties of Inner Hair Cell Cav1.3 $\mathrm{Ca}^{2+}$ channels" Göttingen/ Germany/ Sept. 2013

Nanotechnology in Biology and Medicine: MM. Picher, S. Küpcü, C. Huang, D. Pum, J. Dostalek, U. Sleytr, P. Ertl: “Anti-fouling Layers for on-chip blood analysis " Krems/ Austria/ Nov. 2010

Talks:

Deutsche Gesellschaft für Audiologie DGA: "Die Rolle von $\mathrm{Ca}^{2+}$ bindeprotein 2 in der synaptischen Schallkodierung" Bochum/ Germany/ Sep. 2015

DFG Thematic conference on ultrafast and temporally precise information processing : "The Role of Calcium Binding Protein 2 in Synaptic Sound Encoding and hearing” Bad Münster/ Germany/ Sep. 2014

HUJI-GAU cooperation meeting: "The Role of Calcium Binding Protein 2 in Synaptic Sound Encoding and hearing" Jerusalem/ Israel/ Sep. 2013

DFG Thematic conference on ultrafast and temporally precise information processing : "The Role of Calcium Binding Protein 2 in Synaptic Sound Encoding and hearing” Volkenroda/ Germany/ Sep. 2013

DFG Kickoff-Meeting: “The Role of Calcium binding proteins in Sound Encoding” Kaiserslautern/ Germany/ Aug. 2012

External Group seminar in Iowa: "The Role of Calcium Binding Protein 2 in Synaptic Sound Encoding" USA/ Iowa City/ Feb. 2012

\section{Publications}

Picher MM Küpcü S, Huang C, Dostalek J, Pum D, Sleytr U, Ertl P. "Nanobiotechnology advanced antifouling surfaces for the continuous electrochemical monitoring of glucose in whole blood using a lab-on-a-chip", Lab. Chip 13, 1780-1789 (2013)

Schrauwen I, Helfmann S, Inagaki A, Predoehl F, Tabatabaiefar MA, Picher MM Sommen M, Seco CZ, Oostrik J, Kremer H, Dheedene A, Claes C, Fransen E, Chaleshtori MH, Coucke P, Lee A, Moser T, Van Camp G. "A mutation in $\mathrm{Ca}^{2+}$ binding protein 2, expressed in cochlear hair cells, causes autosomal recessive hearing impairment", Am. J. Hum. Genet. 91, 636-645 (2012)

Jung S, Oshima-Takago T, Chakrabarti R, Wong AB, Jing Z, Yamanbaeva G, Picher MM, Wojcik SM, Göttfert F, Predoehl F, Michel K, Hell SW, Schoch S, Strenzke N, Wichmann C, Moser T." Rab3-interacting molecules $2 \alpha$ and $2 \beta$ promote the abundance of voltage-gated Cav1.3 $\mathrm{Ca}^{2+}$ channels at hair cell active zones", Proc. Natl. Acad. Sci. U. S. A. 112, E3141-3149 (2015) 